\title{
Early-Time Observations of Gamma-Ray Burst Error Boxes with the Livermore Optical Transient Imaging System
}

\author{
G.G. Williams
}

Ph.D

\section{August 1, 2000}

U.S. Department of Energy

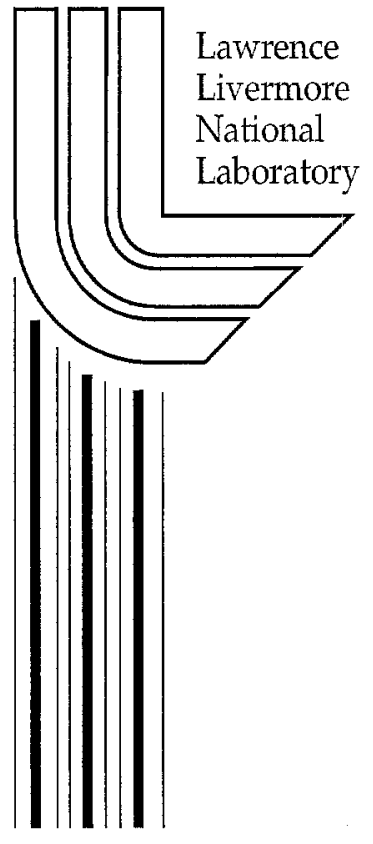




\section{DISCLAIMER}

This document was prepared as an account of work sponsored by an agency of the United States Government. Neither the United States Government nor the University of California nor any of their employees, makes any warranty, express or implied, or assumes any legal liability or responsibility for the accuracy, completeness, or usefulness of any information, apparatus, product, or process disclosed, or represents that its use would not infringe privately owned rights. Reference herein to any specific commercial product, process, or service by trade name, trademark, manufacturer, or otherwise, does not necessarily constitute or imply its endorsement, recommendation, or favoring by the United States Government or the University of California. The views and opinions of authors expressed herein do not necessarily state or reflect those of the United States Government or the University of California, and shall not be used for advertising or product endorsement purposes.

This work was performed under the auspices of the U. S. Department of Energy by the University of California, Lawrence Livermore National Laboratory under Contract No. W-7405-Eng-48.

This report has been reproduced directly from the best available copy.

Available electronically at http://www.doe.gov/bridge

Available for a processing fee to U.S. Department of Energy

and its contractors in paper from

U.S. Department of Energy

Office of Scientific and Technical Information

P.O. Box 62

Oak Ridge, TN 37831-0062

Telephone: (865) 576-8401

Facsimile: (865) 576-5728

E-mail: reports@adonis.osti.gov

Available for the sale to the public from

U.S. Department of Commerce

National Technical Information Service

5285 Port Royal Road

Springfield, VA 22161

Telephone: (800) 553-6847

Facsimile: (703) 605-6900

E-mail: orders@ntis.fedworld.gov

Online ordering: http://www.ntis.gov/ordering.htm

OR

Lawrence Livermore National Laboratory

Technical Information Department's Digital Library

http://www.llnl.gov/tid/Library.html 


\section{EARLY-TIME OBSERVATIONS OF GAMMA-RAY BURST ERROR BOXES WITH THE LIVERMORE OPTICAL TRANSIENT IMAGING SYSTEM}

A Dissertation
Presented to
the Graduate School of
Clemson University
In Partial Fulfillment
of the Requirements for the Degree
Doctor of Philosophy
Physics
by
George Grant Williams
August 2000

Advisor: Dr. Dieter H. Hartmann 


\begin{abstract}
Despite the enormous wealth of gamma-ray burst (GRB) data collected over the past several years the physical mechanism which causes these extremely powerful phenomena is still unknown. Simultaneous and early time optical observations of GRBs will likely make an great contribution to our understanding.

LOTIS is a robotic wide field-of-view telescope dedicated to the search for prompt and early-time optical afterglows from gamma-ray bursts. LOTIS began routine operations in October 1996 and since that time has responded to over 145 gamma-ray burst triggers. Although LOTIS has not yet detected prompt optical emission from a GRB its upper limits have provided constraints on the theoretical emission mechanisms.

Super-LOTIS, also a robotic wide field-of-view telescope, can detect emission 100 times fainter than LOTIS is capable of detecting. Routine observations from Steward Observatory's Kitt Peak Station will begin in the immediate future. During engineering test runs under bright skies from the grounds of Lawrence Livermore National Laboratory Super-LOTIS provided its first upper limits on the early-time optical afterglow of GRBs.

This dissertation provides a summary of the results from LOTIS and SuperLOTIS through the time of writing. Plans for future studies with both systems are also presented.
\end{abstract}




\section{DEDICATION}

To my family; mom, dad, Amy and Kyle. Thank you for all the support and encouragement throughout my studies and my life. 


\section{ACKNOWLEDGMENTS}

I would like to express my sincere thanks to Dr. Dieter Hartmann for his guidance during this work.

Hye-Sook Park of Lawrence Livermore National Lab acted as a second research advisor. Her enthusiasm and dedication to this experiment provided the foundation on which it operated.

This work would not have been possible without excellent work of Elden Ables, Eric Parker and Linda Ott. Their support with the electronics and software kept the experiment operational for three years without any significant down time.

Thanks also to Rodin Porrata who put in tireless hours toward a successful experiment. The entire LOTIS collaboration including Richard Bionta, Paul Butterworth, Tom Cline, Don Ferguson, Gerry Fishman, Neil Gehrels, Chryssa Kouveliotou, Chip Meegan, Bob Nemiroff, and Tim Sasseen contributed to this work. Specific thanks go to David Band who provided all the spectral fits, Scott Barthelmy who provided the GCN triggers and a great effort in implementing Super-LOTIS, Kevin Hurley for accommodating my personal requests for IPN data, and R. Marc Kippen who provided BATSE data as well as the probability analysis software.

Jan and Eric Parker provided a home away from home during my stays in Livermore.

I would also like to thank Lihsin The for the computer support, Jason Brown for the afternoon coffee, and Inger Marie Bjølseth for patience and understanding.

Finally, this work has been followed, supported and supplemented by all my committee members Dr. Donald Clayton, Dr. Mark Leising, and Dr. Bradley Meyer. All of the committee members were very helpful with suggestions and in building my knowledge of astrophysics. 


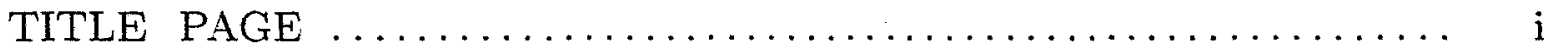

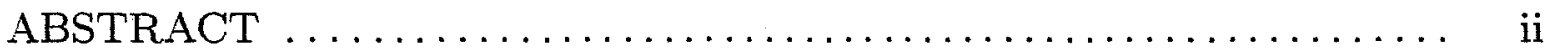

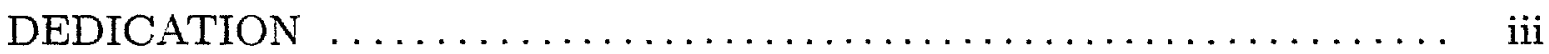

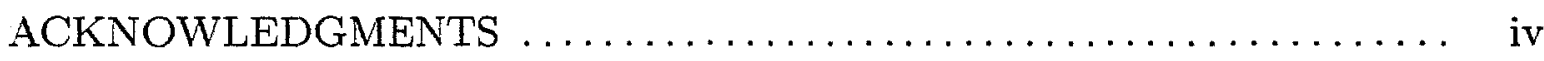

LIST OF TABLES $\ldots \ldots \ldots \ldots \ldots \ldots \ldots \ldots \ldots \ldots \ldots \ldots \ldots \ldots \ldots \ldots \ldots \ldots \ldots$ vii

LIST OF FIGURES $\ldots \ldots \ldots \ldots \ldots \ldots \ldots \ldots \ldots \ldots \ldots \ldots \ldots \ldots \ldots \ldots \ldots \ldots$ viii CHAPTER

1. INTRODUCTION $\ldots \ldots \ldots \ldots \ldots \ldots \ldots \ldots \ldots \ldots \ldots \ldots \ldots \ldots \ldots \ldots \ldots$

1.1 Gamma-Ray Bursts $\ldots \ldots \ldots \ldots \ldots \ldots \ldots \ldots \ldots \ldots \ldots \ldots \ldots \ldots$

1.2 GRB History and Characteristics $\ldots \ldots \ldots \ldots \ldots \ldots \ldots \ldots \ldots \ldots \ldots \ldots$

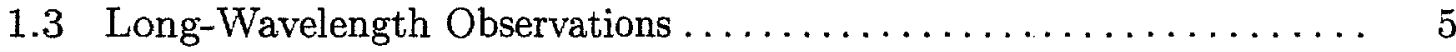

1.4 Current State .................................. 9

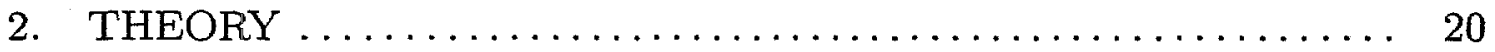

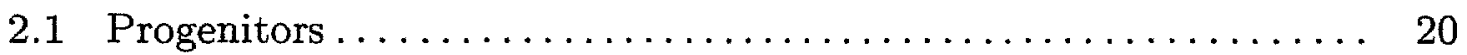

2.2 Fireballs and Relativistic Blast Waves $\ldots \ldots \ldots \ldots \ldots \ldots \ldots \ldots .22$

2.3 Internal Shocks and GRBs $\ldots \ldots \ldots \ldots \ldots \ldots \ldots \ldots \ldots \ldots \ldots \ldots \ldots \ldots \ldots \ldots, 23$

2.4 External Forward Shocks and Afterglows .................. 24

2.5 External Reverse Shocks and Prompt Emission .............. 26

3. EXPERIMENTAL SETUP $\ldots \ldots \ldots \ldots \ldots \ldots \ldots \ldots \ldots \ldots \ldots \ldots \ldots \ldots \ldots \ldots, 29$

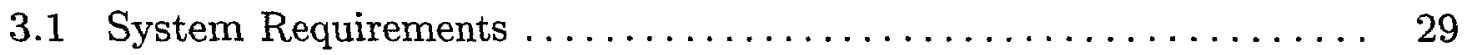

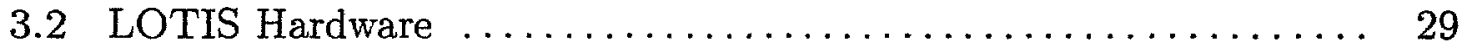

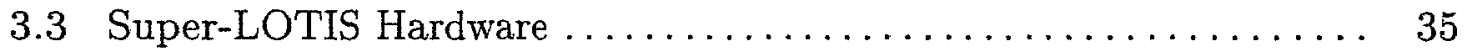

3.4 Camera Calibration $\ldots \ldots \ldots \ldots \ldots \ldots \ldots \ldots \ldots \ldots \ldots \ldots \ldots \ldots \ldots \ldots \ldots, 37$

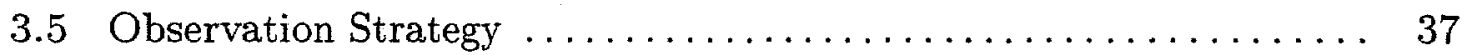

3.5.1 Sky Patrol .................................. 37

3.5.2 Gamma-Ray Burst Triggers $\ldots \ldots \ldots \ldots \ldots \ldots \ldots \ldots \ldots \ldots$

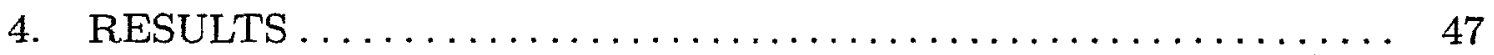

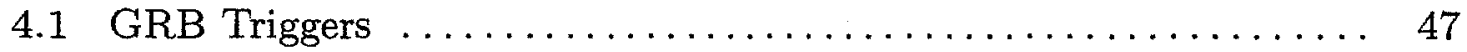

4.2 Data Presentation $\ldots \ldots \ldots \ldots \ldots \ldots \ldots \ldots \ldots \ldots \ldots \ldots \ldots, 47$

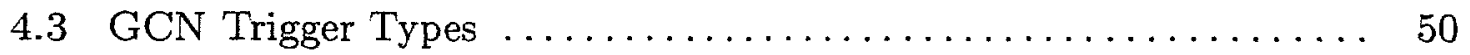

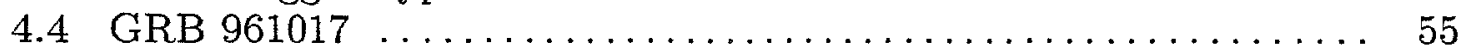


Table of Contents (Continued)

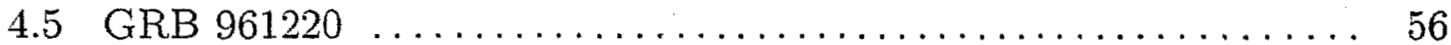

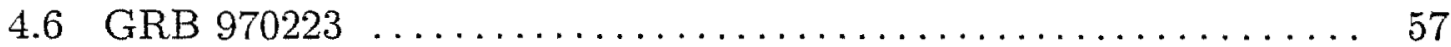

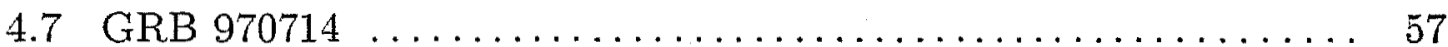

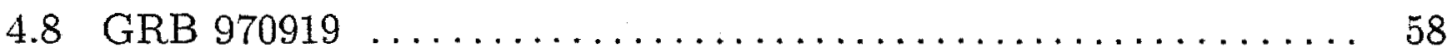

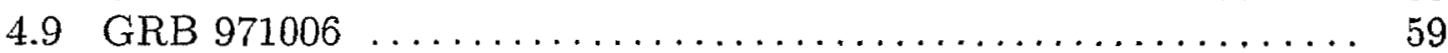

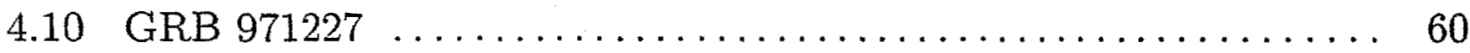

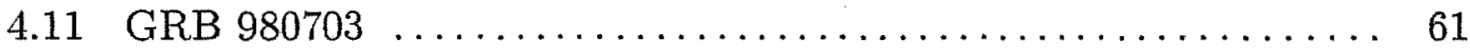

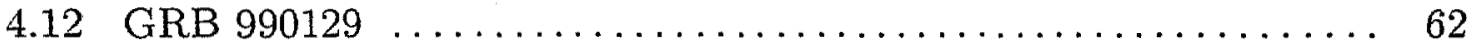

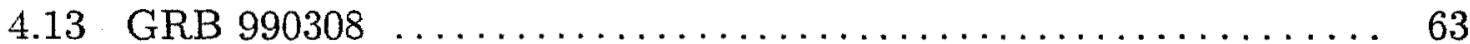

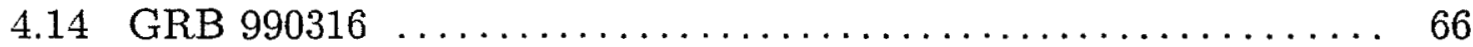

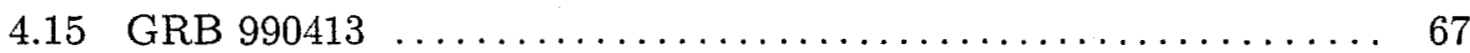

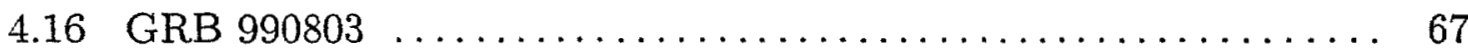

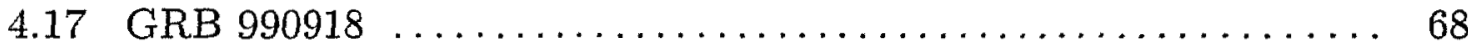

5. DATA ANALYSIS AND INTERPRETATION ................ 101

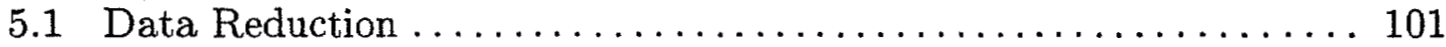

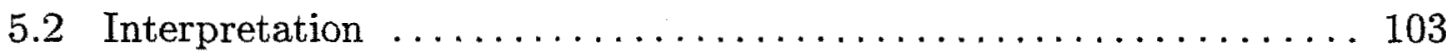

5.2 .1 Scaled Burst Properties .......................... 105

$5.2 .2 \quad$ Spectral Extrapolation ......................... 107

5.2 .3 Reverse Shock Constraints $\ldots \ldots \ldots \ldots \ldots \ldots \ldots \ldots \ldots \ldots \ldots$

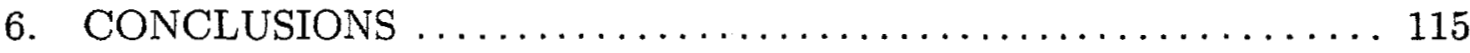

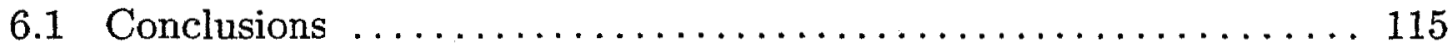

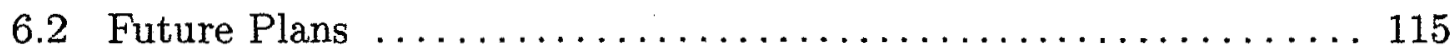

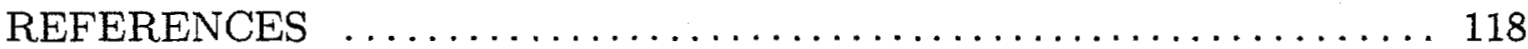




\section{LIST OF TABLES}

Table $\quad$ Page

I. Localized GRBs. ................................. 11

II. LOTIS GRB events with good coverage.................. 52

III. GCN Trigger Types. ............................. 54

IV. LOTIS GRB events scaled to GRB $990123 \ldots \ldots \ldots \ldots \ldots \ldots \ldots \ldots$

V. Optical flux scaling factors. ....................... 109 


\section{LIST OF FIGURES}

Figure $\quad$ Page

1.1 The Compton Gamma-Ray Observatory. ............... 3

1.2 A single BATSE module. . . . . . . . . . . . . $3 \ldots \ldots \ldots \ldots$

1.3 Final BATSE gamma-ray burst skymap. ............... 4

1.4 Example GRB light curves. . . . . . . . . . $5 \ldots \ldots \ldots \ldots \ldots \ldots \ldots$

1.5 Distribution of BATSE gamma-ray burst durations. . . . . . . . . 6

1.6 GRB spectral evolution. . . . . . . .

1.7 GRB 990510 multiband afterglow lightcurve. . . . . . . . . . . 16

1.8 Lightcurve of GRB $990123 \ldots \ldots \ldots \ldots \ldots \ldots \ldots \ldots \ldots \ldots \ldots \ldots \ldots \ldots \ldots$

1.9 GRB publications per year. . . . . . . . . . . . . . . . 19

2.1 Synchrotron spectrum for the cases of fast and slow cooling

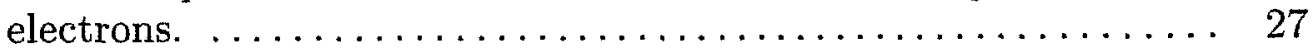

2.2 Relativistic blast wave schematic. $\ldots \ldots \ldots \ldots \ldots \ldots \ldots \ldots \ldots \ldots$

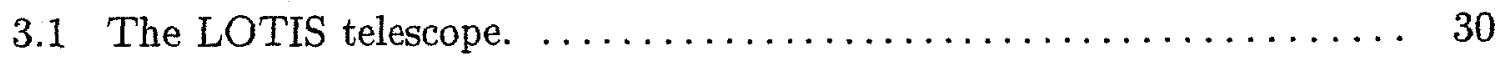

3.2 Optical design of the LOTIS Canon EF $200 \mathrm{~mm}$ Lens. . . . . . . . . . 31

3.3 Cut away diagram of the Loral 443 CCD $\ldots \ldots \ldots \ldots \ldots \ldots \ldots \ldots \ldots$

3.4 LOTIS camera readout electronics design. ............... 33

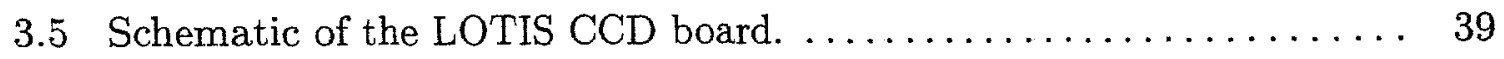

3.6 Schematic of the LOTIS ADC board. ................. 40

3.7 Schematic of the LOTIS control board. . . . . . . . . . . . 41

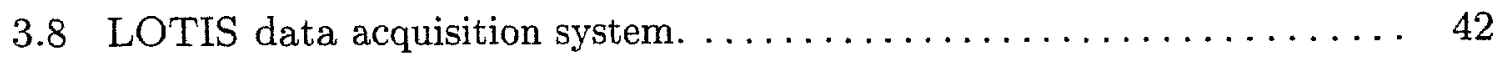

3.9 The Super-LOTIS telescope. . . . . . . . . . . . . . 43

3.10 Design of the Super-LOTIS coma corrector................ 44

3.11 Super-LOTIS coma corrector optical light path. ............ 45

3.12 Loral 442A CCD dark current calibration data. ............. 46

4.1 Histogram of LOTIS response times. ................. 48 
4.2 Histogram of errors between the Original GCN coordinates and the final Huntsville coordinates. . . . . . . . . . . . . . . . 49

4.3 Histogram of GRB durations for LOTIS events. . . . . . . . . . 50

4.4 Histogram of $1 \sigma$ error radii for LOTIS events. . . . . . . . . . . 51

4.5 The BATSE burst location error model. ................ 53

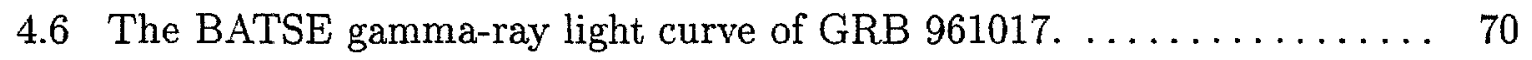

4.7 LOTIS coverage of GRB $961017 \ldots \ldots \ldots \ldots \ldots \ldots \ldots \ldots \ldots \ldots \ldots \ldots$

4.8 The BATSE gamma-ray light curve of GRB $961220 \ldots \ldots \ldots \ldots \ldots \ldots 72$

4.9 LOTIS coverage of GRB $961220 \ldots \ldots \ldots \ldots \ldots \ldots \ldots \ldots \ldots \ldots \ldots$

4.10 The BATSE gamma-ray light curve of GRB $970223 \ldots \ldots \ldots \ldots \ldots \ldots$

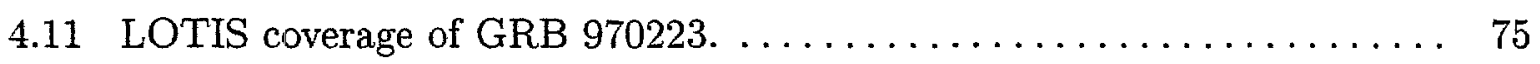

4.12 The BATSE gamma-ray light curve of GRB $970714 \ldots \ldots \ldots \ldots \ldots$

4.13 LOTIS coverage of GRB $970714 \ldots \ldots \ldots \ldots \ldots \ldots \ldots \ldots \ldots \ldots$

4.14 The BATSE gamma-ray light curve of GRB $970919 \ldots \ldots \ldots \ldots \ldots \ldots$

4.15 LOTIS coverage of GRB $970919 . \ldots \ldots \ldots \ldots \ldots \ldots \ldots \ldots \ldots \ldots$

4.16 The BATSE gamma-ray light curve of GRB $971006 \ldots \ldots \ldots \ldots \ldots$

4.17 LOTIS coverage of GRB $971006 \ldots \ldots \ldots \ldots \ldots \ldots \ldots \ldots \ldots \ldots$

4.18 The BATSE gamma-ray light curve of GRB $971227 \ldots \ldots \ldots \ldots \ldots \ldots$

4.19 LOTIS coverage of GRB $971227 \ldots \ldots \ldots \ldots \ldots \ldots \ldots \ldots \ldots \ldots$

4.20 Broadband light curve of GRB $971227 \ldots \ldots \ldots \ldots \ldots \ldots \ldots \ldots \ldots$

4.21 Optical afterglow light curve of GRB $980703 \ldots \ldots \ldots \ldots \ldots \ldots \ldots$

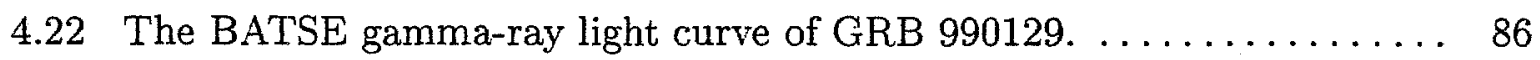

4.23 LOTIS coverage of GRB 990129 ; first image. . . . . . . . . . . . 87

4.24 LOTIS coverage of GRB 990129 ; seventh image. . . . . . . . . . 88

4.25 The BATSE gamma-ray light curve of GRB 990308. . . . . . . . . 89

4.26 LOTIS coverage of GRB 990308; first image. . . . . . . . . . . 90

4.27 LOTIS coverage of GRB 990308; sixth image. . . . . . . . . . . . 91

4.28 Super-LOTIS coverage of GRB 990308 ; wide field. . . . . . . . . . . . 92

4.29 Super-LOTIS coverage of GRB 990308; narrow field. . . . . . . . . . . 93 
List of Figures (Continued) $\quad$ Page

4.30 The BATSE gamma-ray light curve of GRB $990316 \ldots \ldots \ldots \ldots \ldots \ldots 9$

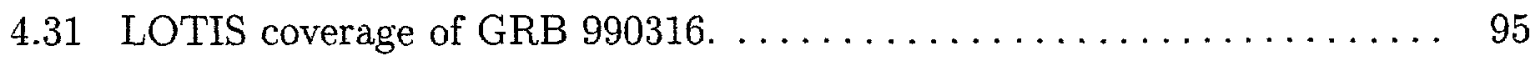

4.32 The BATSE gamma-ray light curve of GRB $990413 \ldots \ldots \ldots \ldots \ldots \ldots 96$

4.33 LOTIS coverage of GRB $990413 \ldots \ldots \ldots \ldots \ldots \ldots \ldots \ldots \ldots \ldots$

4.34 The BATSE gamma-ray light curve of GRB 990803. . . . . . . . 98

4.35 The BATSE gamma-ray light curve of GRB $990918 \ldots \ldots \ldots \ldots \ldots \ldots$.

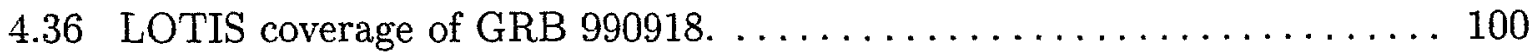

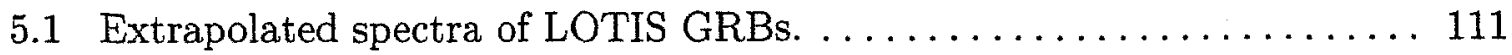

5.2 Magnitude of the prompt optical emission. ............... 112

5.3 Prompt optical synchrotron spectra. . . . . . . . . . . . . 113

5.4 Prompt optical emission from the reverse shock. . . . . . . . . . 114

6.1 Upper limits of the prompt optical emission from GRBs. . . . . . . . 117 


\section{CHAPTER 1 \\ INTRODUCTION}

\section{Gamma-Ray Bursts}

Cosmic gamma-ray bursts (GRBs) are short, typically $0.1 \mathrm{~s}-100 \mathrm{~s}$, flashes of predominantly gamma-ray radiation which occur at unpredictable times from random directions. Although many bursts go undetected, GRBs occur at a rate of at least three times per day (threshold dependent; e.g., Fishman \& Hartmann 1997). In the short time a GRB is active it often out-shines all other sources of gamma-rays in the sky combined (Fishman 1995).

Perhaps the most fundamental question is: What astrophysical phenomena causes these events? Currently the answer is not definitively known. Many hypotheses exist, most of which involve a cataclysmic one-time event. The research described in this work was directed at narrowing these hypotheses by acquiring early-time optical data that was difficult to obtain previously. Before describing the work it is appropriate to review the current state of GRB knowledge. More complete overviews of GRBs are presented in Fishman \& Meegan (1995) and Piran (1999a).

\section{GRB History and Characteristics}

The first published observations of gamma-ray bursts of cosmic origin came from Klebesadel et al. in 1973. This pioneering work describes 16 bursts detected by the Vela spacecraft in the time period spanning 1969-1972. Before their discovery, Colgate (1968) had suggested that detectable gamma-rays would be emitted from supernova (SN) explosions. This possibility was addressed in the discovery paper although no definitive association with a nova or supernova could be found.

In the years which followed, several other spacecraft and balloon-borne detectors provided additional information about the characteristics of GRBs (e.g., Cline et al. 1973; Herzo et al. 1976; Cline et al. 1979; Gilman et al. 1980; Schwartz et al. 1987). The data were accompanied by numerous models attempting to explain the 
origin of GRBs (e.g., Grindlay \& Fazio 1974; Hoyle \& Clayton 1974; Woosley \& Wallace 1982; Holcomb \& Tajima 1991). See Ruderman (1975) for a review of early models. Many early models assumed a Galactic origin of GRBs since the measured fluxes would require an enormous energy release if they originated in external galaxies. Galactic models were also supported by evidence of GRB spectral line features which were interpreted as cyclotron lines produced in intense magnetic fields near neutron stars (e.g., Mazets et al. 1981; Murakami et al. 1988; Wang et al. 1989). However the early sky distributions appeared uniform rather than concentrated in the Galactic plane, perhaps suggesting that the detectors were only sampling the nearby events (e.g., Hartmann \& Epstein 1989).

The 5 April 1991 launch of the second of NASAs Great Observatories, the Compton Gamma-Ray Observatory (CGRO; Figure 1.1), greatly increased the GRB data accumulation rate. The Burst and Transient Source Experiment (BATSE) was designed in part as an all sky monitor to detect GRBs. The instrument consists of 8 modules, shown in Figure 1.2, mounted on the corners of CGRO. Each module consists of two NaI detectors; a Large Area Detector (LAD) optimized for sensitivity and directional reconstruction, and a Spectroscopy Detector (SD) optimized for energy coverage and energy resolution (Fishman et al. 1992). The SDs, sensitive from $\sim 60 \mathrm{keV}-\sim 11 \mathrm{MeV}$, have a circular detector area of $127 \mathrm{~cm}^{2}$ and a thickness of $7.6 \mathrm{~cm}$ (Schaefer et al. 1994b). The LADs, sensitive to photon energies of $\sim 25 \mathrm{keV}-\sim 2 \mathrm{MeV}$, have a diameter of $50.8 \mathrm{~cm}$ and a thickness of $1.27 \mathrm{~cm}$. The LADs generate a burst trigger when two or more detectors register a significant $(\sim 5 \sigma)$ increase in the background in any one of three time intervals; $64 \mathrm{~ms}, 256 \mathrm{~ms}$, or $1024 \mathrm{~ms}$ (Fishman et al. 1994).

The recent failure of one of the CGRO gyros led to a decision based on safety to de-orbit the spacecraft. The spacecraft reentered the Earth's atmosphere on June 4, 2000. During its approximate 9 year lifetime BATSE recorded 8021 triggers: 2704 GRBs, 1190 solar flares, 1717 magnetospheric events, 76 terrestrial gamma-ray flashes, 1999 transient sources, 184 soft gamma-ray repeaters, 35 phosphorescence spikes, 33 unknown events, 56 commanded or accidental events, and 27 events with insufficient 


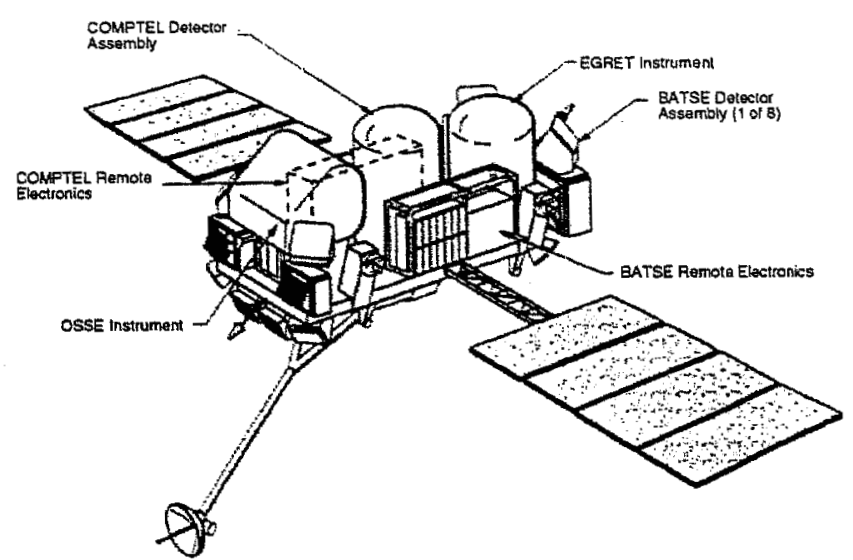

Figure 1.1 The Compton Gamma-Ray Observatory.

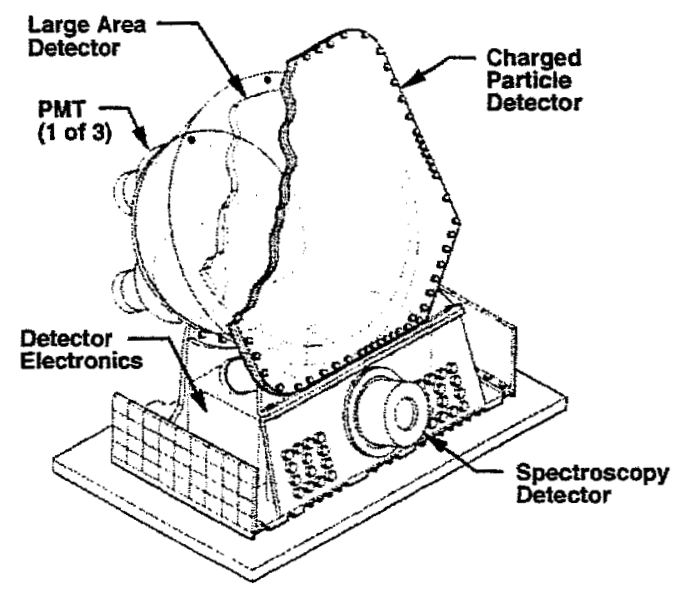

Figure 1.2 A single BATSE module.

data. A great deal of the current knowledge about GRBs was acquired through BATSE measurements. Figure 1.3 shows the locations of the 2704 GRBs plotted in galactic coordinates.

GRBs have durations which range from milliseconds to hundreds of seconds. Their light curves do not conform to a single shape although fast rising exponential decays (FREDs) are common. Variability time-scales can be as rapid as less than a 


\section{BATSE Gamma-Ray Bursts}

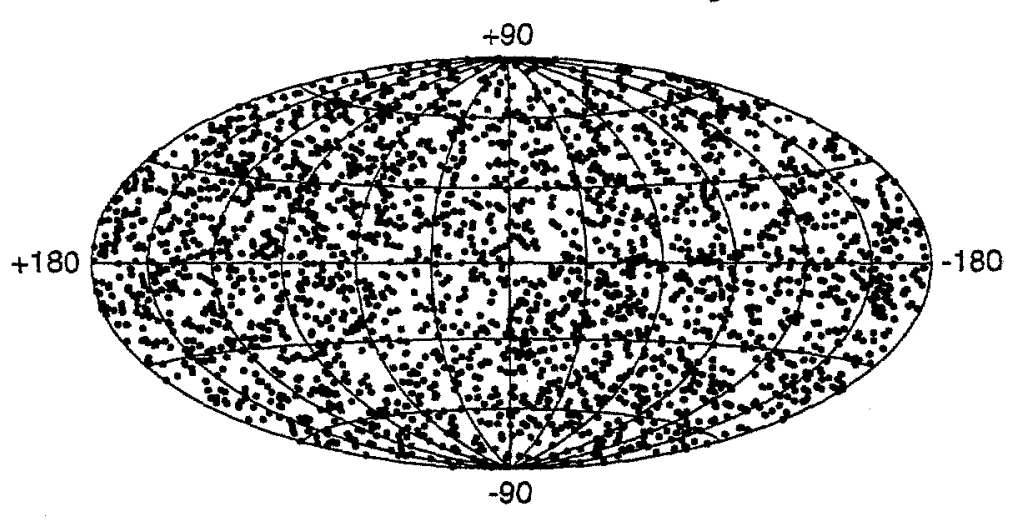

Figure 1.3 Final BATSE gamma-ray burst skymap.

millisecond (e.g., Walker et al. 2000). Figure 1.4 gives four examples of GRB light curves detected by BATSE.

Figure 1.5 shows a plot of histograms of GRB light curve durations. The BATSE team uses two measures of the burst duration, $T_{90}$ and $T_{50} . T_{90}\left(T_{50}\right)$ is defined as the time interval encompassing $90 \%(50 \%)$ of the total GRB counts, i.e. the time during which the integrated counts increases from $5 \%(25 \%)$ to $95 \%(75 \%)$ above background (Fishman et al. 1994). Figure 1.5 shows an obvious bimodal distribution of GRB durations which may separate the events into two classes physically (Kouveliotou et al. 1993).

The time average spectrum of most gamma-ray bursts is well fitted by the functional form suggested by Band et al. (1993),

$$
N_{E}(E)= \begin{cases}A\left(\frac{E}{100 \mathrm{keV}}\right)^{\alpha} \exp \left(-\frac{E}{E_{0}}\right), & (\alpha-\beta) E_{0} \geq E \\ A\left[\frac{(\alpha-\beta) E_{0}}{100 \mathrm{keV}}\right]^{\alpha-\beta} \exp (\beta-\alpha)\left(\frac{E}{100 \mathrm{keV}}\right)^{\beta}, & (\alpha-\beta) E_{0} \leq E\end{cases}
$$

where $A$ is the amplitude in photons $\mathrm{cm}^{-2} \mathrm{~s}^{-1} \mathrm{keV}^{-1}$. The Band Function, as it is termed, is a broken power law with a low energy index of $\alpha$, a high energy index of $\beta$, and a break energy $E_{0}$, which is typically near a few hundred keV. A second characteristic energy which is often referred to in the literature is the peak energy of the $E^{2} N(E)$ (or $\nu F_{\nu}$ ) power spectrum. The two energies are related by $E_{p}=(\alpha+2) E_{0}$. 
Although not universally true, it is common for $E_{p}$ in time resolved spectra to increase with increases in instantaneous burst intensity (Golenetskii et al. 1983; Ford et al. 1995) and for a general hard-to-soft trend over the entire burst (Norris et al. 1986). Crider et al. (1997) also found a positive correlation between the low-energy powerlaw slope, $\alpha$, and $E_{p}$. Figure 1.6 gives an example of the spectral evolution of a GRB (Crider et al. 1997). The beginning times are indicated to the right of the individual spectra. The evolution of $\alpha$ and $E_{p}$ are show as insets.
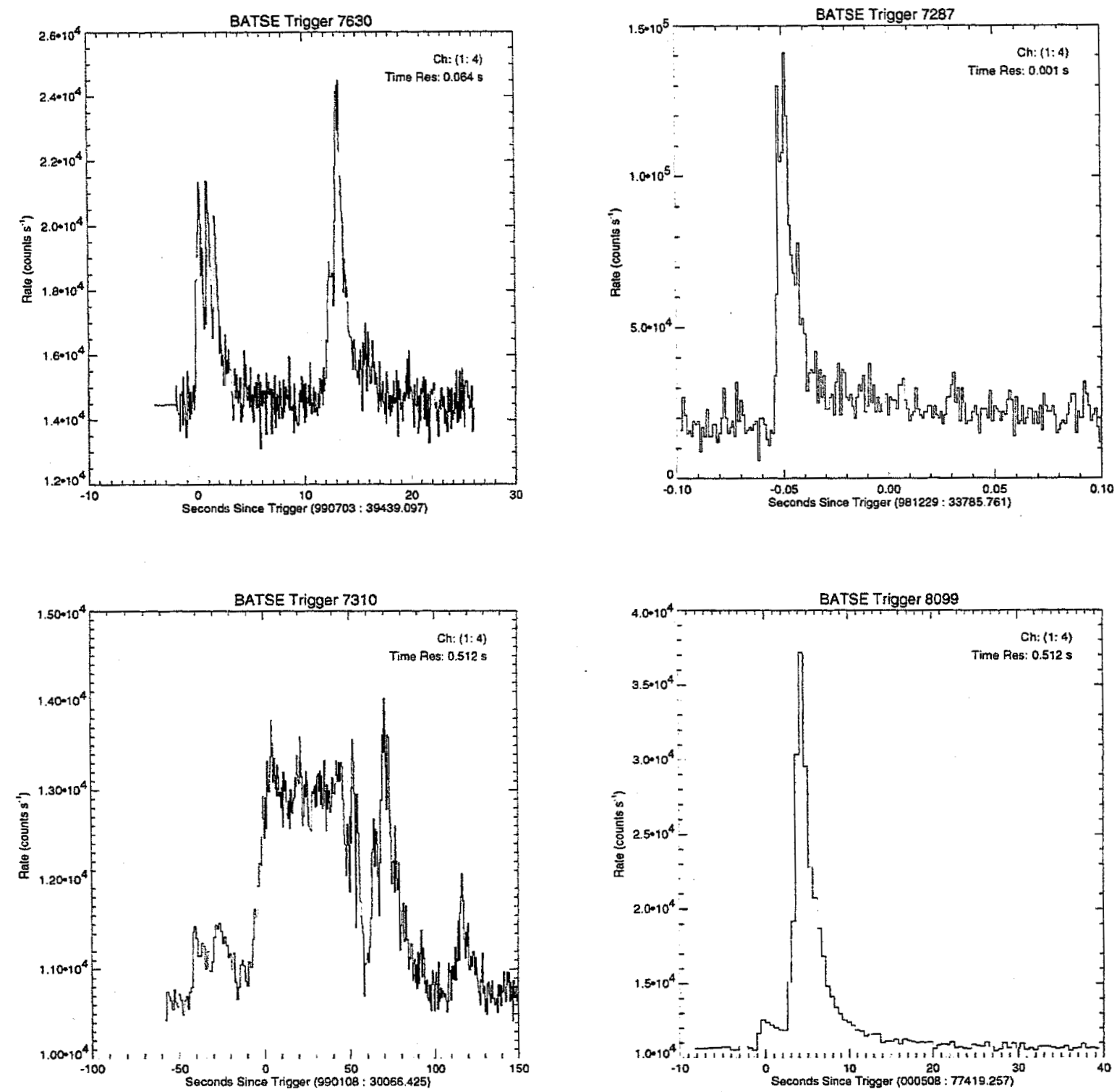

Figure 1.4 Example GRB light curves. 


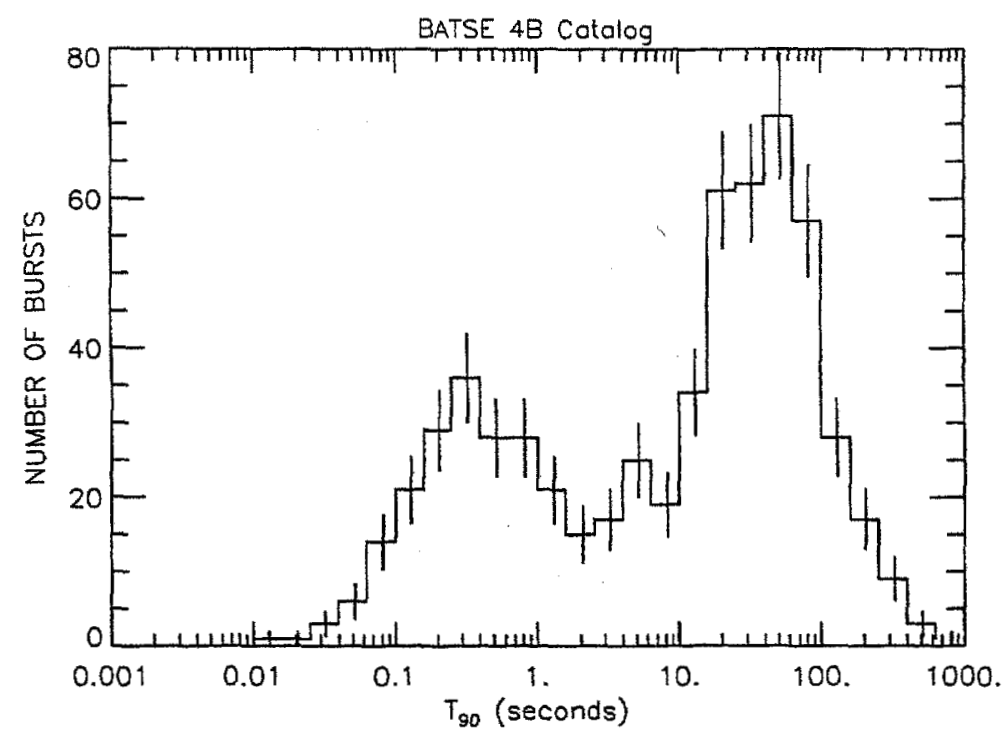

Figure 1.5 Distribution of BATSE gamma-ray burst durations (4B Catalog).

\section{Long-Wavelength Observations}

Since gamma-rays are difficult to focus, GRB locations cannot be precisely determined. Hence there was little chance of determining the source of GRBs from the gamma-ray data alone. Long-wavelength counterparts which could pinpoint the burst location have been the focus of numerous searches (e.g., Schaefer 1981; Cortiglioni et al. 1981; Hjellming \& Ewald 1981; Fishman et al. 1981; Hudec et al. 1987). If a counterpart were identified, follow-up observations with larger telescopes could reveal an association with a Galactic object or a distant host galaxy. This type of measurement might definitively place GRBs within the Milky Way or at cosmological distances.

Although many attempts were made to identify long-wavelength counterparts, it remained unclear whether low energy emission accompanied the bursts. Ford \& Band (1996) found that a simple extrapolation of the GRB spectra would yield only a few observable flaring optical events per year to a limiting magnitude of $\mathrm{m}=10-15$. The brighter predicted optical fluxes would result from bursts with soft gamma-ray spectra. However it is unlikely that the physical processes responsible for the gammaray emission could also produce optical photons. 


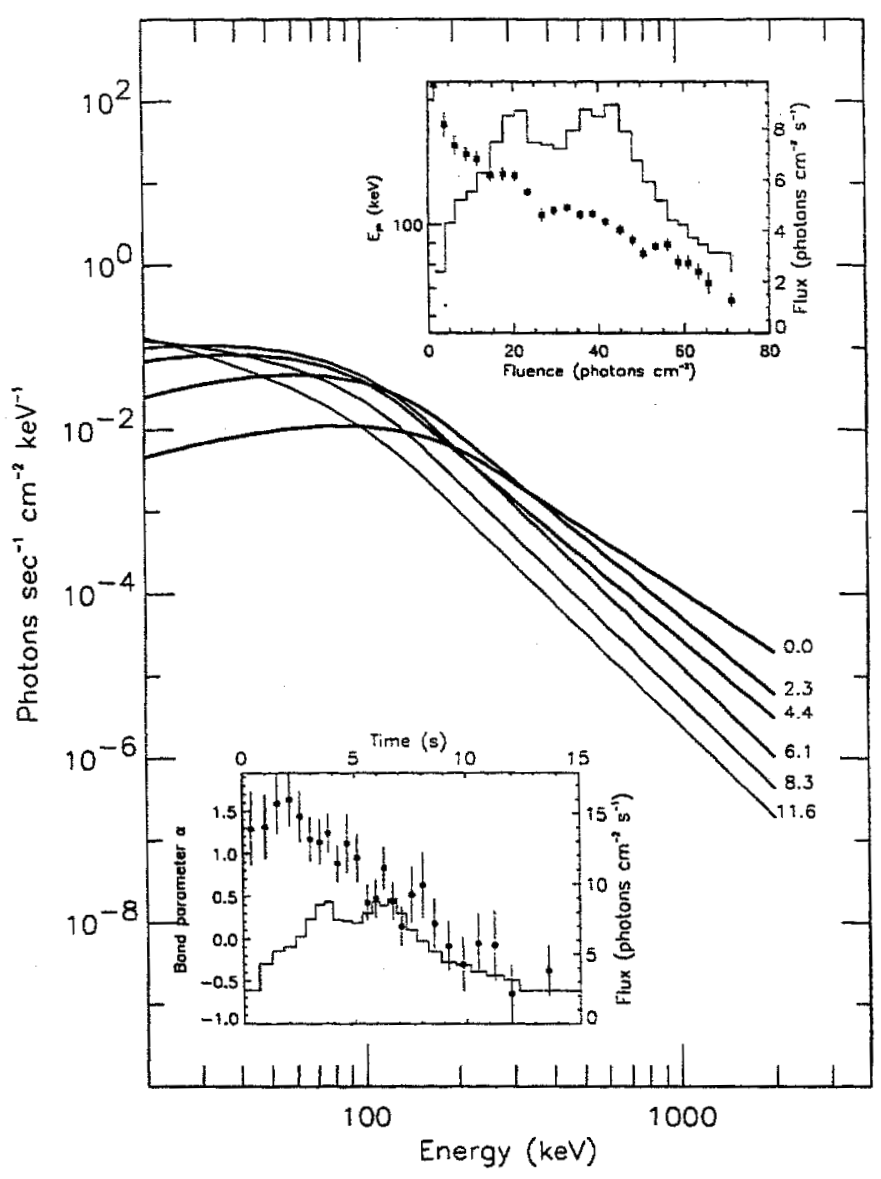

Figure 1.6 GRB spectral evolution of GRB 910927. The beginning times are labeled to the right of each Band function fit. The insets show the evolution of $E_{p}$ and photon flux with fluence (upper right) and the low energy power law tail $\alpha$ and photon flux with time (lower) left (Crider et al. 1997).

Here we review only searches for optical counterparts. These counterparts might come in the form of quiescent counterparts detectable in deep searches long after the burst occurs, repeating outbursts detectable in archival plate searches, or flaring or fading events detectable during simultaneous or early-time observations. More complete reviews of early searches for optical counterparts are presented in Hudec (1993a) and Hudec (1995). Reviews of the history of all long-wavelength searches are presented in Greiner (1995), Hartmann (1995), Vrba (1996), and CastroTirado (1998). 
Some GRB optical counterpart searches assumed that GRB source outbursts repeated. Archival plates were inspected for transient phenomena or common quiescent counterparts within well localized GRB error boxes (Schaefer 1981; Schaefer et al. 1984; Atteia et al. 1985; Hudec et al. 1987; Moskalenko et al. 1989; Greiner et al. 1987; Greiner \& Motch 1995). Schaefer (1981) was the first to claim the discovery of such an optical transient. Several additional candidates followed (Schaefer et al. 1984; Scholz 1984; Moskalenko et al. 1989; Greiner \& Motch 1995; Hudec et al. 1994). Greiner et al. (1990), Zytkow (1990), and Greiner (1992) found that many of these candidates could be attributed to plate defects. In addition soft gamma-ray repeaters rather than classical GRBs accounted for some of the error boxes which were searched.

Deep observations were also carried out on several GRB error boxes long after the burst occurred. Because of the large delay between the burst and the observation these searches were directed towards quiescent counterparts rather than flaring or fading transients. Chevalier et al. (1981), Fishman et al. (1981), Pedersen et al. (1982), Schaefer \& Ricker (1983), Schaefer et al. (1983), Pedersen et al. (1983), Ricker et al. (1989), Harrison et al. (1994a), Harrison et al. (1994b), Vrba et al. (1995), and Sokolov et al. (1996) conducted deep searches of localized GRBs. The Hubble Space Telescope was also used by Schaefer et al. (1997) to search for objects in the error boxes of GRB 790113, GRB 790325, GRB 790406, GRB 790613, and GRB 920406. Unique objects within the error boxes were noted but no common counterpart was uncovered. Observations of GRB 930131, the so called "Superbowl Burst", provided one of the earliest rapid follow-up campaigns to significant limiting magnitudes (Schaefer et al. 1994a; McNamara \& Harrison 1994).

Rapid follow-up observations in the past were rare because of the difficulty in localizing bursts fast enough. However serendipitous simultaneous or near simultaneous observations did occur on several occasions. The analysis of these events focused on finding optical transients or fading or flaring sources within the error boxes. The first simultaneous observation was reported by Grindlay et al. (1974). Additional simultaneous observations included Hudec et al. (1987), Hudec (1993b), and Greiner 
et al. (1996b). The low sensitivity of the large field-of-view instruments used for these observations prohibited strong constraints of the optical emission. The upper limits generally reached only $m \sim 6$.

Prior to the advent of real-time GRB triggers a few experiments such as the Explosive Transient Camera (ETC) attempted to obtain simultaneous observation by covering a large area of the sky (e.g., Vanderspek et al. 1992; Krimm et al. 1996) or by responding to optical transients detected by other instruments (e.g., Rapidly Moving Telescope Barthelmy et al. 1992). The BATSE Gamma-Ray Burst Coordinates Distribution Network (BACODINE;Barthelmy et al. 1994) provided the real-time triggers which allowed dedicated sensitive systems to come online. Castro-Tirado et al. (1994) followed up several bursts detected by the WATCH experiments on GRANAT and EURECA. These observations resulted in upper limits of $m=18-20$ from 12 to 80 hours after the burst.

The first experiment directly triggered by a GRB detector was the GammaRay Optical Counterpart Search Experiment (GROCSE; Park et al. 1997a; Lee et al. 1997). GROCSE was an automated rapidly slewing wide field-of-view telescope dedicated to the search for optical counterparts of GRBs. Between January 1994 and June 1996 GROCSE imaged 22 GRB error boxes within $30 \mathrm{~s}$ of the start of the burst. The sensitivity of the system was limited by short integrations which were required because the altitude-azimuth mount of the telescope did not allow tracking. No flaring or fading sources were detected to $\mathrm{m}=7.0-8.5$.

The original purpose of rapid response automated robotic telescopes was to provide better localization of GRBs. In recent years this task has been accomplished through detection of hard X-ray emission and networks of spatially separated gammaray burst monitors. The goal of the automated telescopes has shifted to constraining the GRB environment through observations of the prompt optical emission.

\section{Current State}

The launch of the Italian-Dutch X-ray satellite BeppoSAX (Boella et al. 1997) on April 30, 1996 ushered in a new era of long-wavelength follow-up observations 
of GRBs. SAX is equipped with four Narrow Field Instruments (NFIs; Low Energy Concentrator Spectrometer LECS, Medium Energy Concentrator Spectrometer MECS, High Pressure Gas Scintillation Proportional Counter HPGSPC, Phoswich Detection System PDS) which all point in a same direction, and two Wide Field Cameras (WFCs), pointing in diametrically opposed directions perpendicular to the NFI common axis (Piro et al. 1995; Attina' et al. 1995; Boella et al. 1997). The anticoincidence shield of the PDS performs a secondary function of acting as a GRB Monitor (GRBM; Feroci et al. 1997; Amati et al. 1997; Frontera et al. 1997). If a gamma-ray burst is detected by the GRBM the WFC hard X-ray data are inspected for a coincident detection. Bursts observed by the WFC are generally localized to an error box with a radius of less than $10^{\prime}$. This precise localization allows follow-up observations with the NFI as well as sensitive ground based optical telescopes.

The first two SAX/WFC GRB localizations occurred on 20 July 1996 (Piro et al. 1996b) and 11 January 1997 (Costa et al. 1997c). Both of these events were initially localized to an error circle of radius $10^{\prime}$. After further analysis the radii of both the error boxes were reduced to $3^{\prime}$ (In't Zand et al. 1997). X-ray (Piro et al. 1996a; Murakami et al. 1996; Greiner et al. 1996a; Butler et al. 1997), optical (Luginbuhl et al. 1996; Castro-Tirado et al. 1997; Guarnieri et al. 1997), and radio (Frail et al. 1996, 1997) follow-up observations revealed no definitive counterparts, perhaps because of the delay between the burst and the observations. In the case of GRB 960620 all follow-up observations occurred more than month after the burst. The earliest X-ray, optical, and radio observations of the error box of GRB 970111 were obtained $16.4 \mathrm{~h}, 19.0 \mathrm{~h}$, and $52 \mathrm{~h}$ after the burst, respectively.

The third and perhaps most historically significant SAX localization occurred on 28 February 1997 (Costa et al. 1997a). GRB 970228 was detected by the SAX/GRBM and SAX/WFC on February 28.123620 UT and localized to an error circle with radius of $3^{\prime}$. Follow-up observations by the SAX/NFI revealed a previously unknown source within the SAX/WFC error circle. This object faded by a factor of 20 in three days (Costa et al. 1997b). Optical observations by Groot et al. (1997) revealed an optical transient which appeared to be fading and therefore became the most likely 
candidate for the optical counterpart of GRB 970228. Radio observations by Galama et al. (1997) revealed no radio counterpart. The optical source faded according to a power law in time.

Although SAX has made a substantial contribution there are additional instruments which are capable of localizing GRBs. These include the Rossi X-ray Timing Explorer All Sky Monitor and Proportional Counter Array (RXTE/ASM,RXTE/PCA). One additional method of localizing GRBs is triangulation using timing data from two or more spacecraft which have a large spatial separation. This is the localization technique used by Third Interplanetary Network (IPN). Because of the operating conditions of the SAX/WFC only bursts with significant durations can be detected. Therefore only one class of the bimodal distribution of durations have followed-up observations. The IPN does not suffer this restriction and therefore a localization of a "short burst" should come in the very near future.

Prior to 1 July 2000, a total of 72 GRBs had been localized to a sufficiently small error box to warrant rapid follow-up observations with large telescopes. A complete description of the observations and characteristics of the afterglows is beyond the scope of this work. Table I. summarizes the recent GRB localizations. The first three columns indicate the date of the GRB, the size of the GRB error box, and the method used to localize the burst. Error indicates the radius of an equivalent $1 \sigma$ error circle. The next four columns are marked with an $\mathrm{X}$ if there is an IPN annulus (IPN), an X-ray afterglow (XA), an optical afterglow (OA), or a radio afterglow (RA). The last two columns give the power law decay index of the afterglow and the measured redshift. These afterglow characteristics are generally only available if an optical afterglow has been detected. For a regularly updated summary of localized GRBs consult http://www.aip.de/ jcg/grb.html.

Table I.: Localized GRBs.

\begin{tabular}{lcccccccc} 
Date & Method & Error & IPN & XA & OA & RA & Decay Index & z \\
\hline 960720 & SAX & $3^{\prime}$ & & & & &
\end{tabular}


Table I.: GRBs localized to small error boxes. (Continued)

\begin{tabular}{|c|c|c|c|c|c|c|c|c|}
\hline Date & Method & Error & IPN & $\mathrm{XA}$ & $\mathrm{OA}$ & RA & Decay Index & $\mathrm{z}$ \\
\hline 970111 & SAX & $3^{\prime}$ & $\mathrm{X}$ & $\mathrm{X}$ & & & & \\
\hline 970228 & SAX & $3^{\prime}$ & $\mathrm{X}$ & $\mathrm{X}$ & $\mathrm{X}$ & & $-1.10 \pm 0.10$ & 0.695 \\
\hline 970402 & SAX & $3^{\prime}$ & & $\mathrm{X}$ & & & & \\
\hline 970508 & SAX & $3^{\prime}$ & & $\mathrm{X}$ & $\mathrm{x}$ & $\mathrm{X}$ & $-1.17 \pm 0.04$ & 0.835 \\
\hline 970616 & XTE/IPN & $40^{\prime} \times 2^{\prime}$ & $\mathrm{X}$ & $\mathrm{X}$ & & & & \\
\hline 970815 & $\mathrm{XTE}$ & $6^{\prime} \times 3^{\prime}$ & & $\mathrm{X}$ & & & & \\
\hline 970828 & XTE & $2.5 \times 1^{\prime}$ & $\mathrm{X}$ & $\mathrm{X}$ & & $\mathrm{X}$ & & 0.958 \\
\hline 971024 & XTE & $9^{\prime} \times 1^{\prime}$ & & $\mathrm{X}$ & & & & \\
\hline 971214 & SAX & $4^{\prime}$ & $\mathrm{X}$ & $\mathrm{X}$ & $\mathrm{X}$ & & $-1.20 \pm 0.02$ & 3.418 \\
\hline 971227 & SAX & $8^{\prime}$ & $\mathrm{X}$ & $\mathrm{X}$ & & & & \\
\hline 980109 & $\mathrm{SAX}$ & $10^{\prime}$ & & & & & & \\
\hline 980326 & $\mathrm{SAX}$ & $8^{\prime}$ & $\mathrm{X}$ & & $\mathrm{X}$ & & $-2.00 \pm 0.10$ & $\sim 1.0$ \\
\hline 980329 & $\mathrm{SAX}$ & $3^{\prime}$ & $\mathrm{X}$ & $\mathrm{X}$ & $\mathrm{X}$ & $\mathrm{X}$ & $-1.29 \pm 0.19$ & $\sim 2$ \\
\hline 980425 & SAX & $8^{\prime}$ & & $\mathrm{X}$ & $\mathrm{SN}$ & $\mathrm{X}$ & SN & 0.0085 \\
\hline 980515 & SAX & $5^{\prime}$ & & & & & & \\
\hline 980519 & SAX & $3^{\prime}$ & $\mathrm{X}$ & $\mathrm{X}$ & $\mathrm{X}$ & $\mathrm{X}$ & $-2.30 \pm 0.12$ & \\
\hline 980613 & SAX & $4^{\prime}$ & & $\mathrm{X}$ & $X$ & & $-1.30 \pm 0.00$ & 1.096 \\
\hline 980703 & XTE & $4^{\prime}$ & & $\mathrm{X}$ & $\mathrm{X}$ & $\mathrm{X}$ & $-1.17 \pm 0.25$ & 0.966 \\
\hline 981220 & XTE/IPN & $2^{\prime \prime} .5$ & $\mathrm{x}$ & & & & & \\
\hline 981226 & SAX & $6^{\prime}$ & & $\mathrm{X}$ & & $\mathrm{X}$ & & \\
\hline 990123 & SAX & $2^{\prime}$ & $\mathrm{x}$ & $\mathrm{X}$ & $\mathrm{X}$ & $\mathrm{X}$ & $-1.12 \pm 0.03$ & 1.600 \\
\hline 990217 & SAX & $3^{\prime}$ & & & & & & \\
\hline 990308 & XTE/IPN & $48^{\prime} \times 7^{\prime}$ & $\mathrm{X}$ & & $\mathrm{x}$ & & $-1.2 \pm 0.1$ & \\
\hline 990506 & $\mathrm{XTE}$ & $7^{\prime}$ & $\mathrm{X}$ & $\mathrm{X}$ & & $\mathrm{X}$ & & \\
\hline 990510 & SAX & $3^{\prime}$ & $\mathrm{x}$ & $\mathrm{X}$ & $X$ & & $-0.76 \pm 0.01$ & 1.619 . \\
\hline 990520 & SAX & $3^{\prime}$ & & $\mathrm{X}$ & & & & \\
\hline
\end{tabular}


Table I.: GRBs localized to small error boxes. (Continued)

\begin{tabular}{|c|c|c|c|c|c|c|c|c|}
\hline Date & Method & Error & IPN & $\mathrm{XA}$ & $\mathrm{OA}$ & $\mathrm{RA}$ & Decay Index & $z$ \\
\hline 990625 & SAX & $6^{\prime}$ & & & & & & \\
\hline 990627 & SAX & $3^{\prime}$ & & $\mathrm{x}$ & & & & \\
\hline 990704 & SAX & $7^{\prime}$ & $\mathrm{X}$ & $\mathrm{x}$ & & & & \\
\hline 990705 & $\mathrm{SAX}$ & $6^{\prime}$ & $\mathrm{X}$ & $\mathrm{x}$ & & & $-1.68 \pm 0.10$ & \\
\hline 990712 & SAX & $2^{\prime}$ & & & $\mathrm{x}$ & & $-1.03 \pm 0.02$ & 0.430 \\
\hline 990806 & SAX & $2^{\prime}$ & & $\mathrm{X}$ & & & & \\
\hline 990907 & SAX & $8^{\prime}$ & $\mathrm{x}$ & $\mathrm{X}$ & & & & \\
\hline 990908 & SAX & $8^{\prime}$ & & & & & & \\
\hline 991014 & $\mathrm{SAX}$ & $6^{\prime}$ & $\mathrm{X}$ & $\mathrm{x}$ & & & & \\
\hline 991105 & SAX & $5^{\prime}$ & $\mathrm{x}$ & & & & & \\
\hline 991106 & SAX & $4^{\prime}$ & & $\mathrm{X}$ & & & & \\
\hline 991208 & IPN & $14^{\prime} \times 1^{\prime}$ & $\mathrm{X}$ & & $\mathrm{X}$ & $\mathrm{X}$ & $-2.15 \pm 0.0$ & 0.706 \\
\hline 991216 & XTE & $3^{\prime} \times 5^{\prime}$ & $\mathrm{x}$ & $\mathrm{x}$ & $\mathrm{x}$ & $\mathrm{X}$ & $-1.22 \pm 0.04$ & \\
\hline 991217 & SAX & $4 ! 5$ & & & & & & \\
\hline 000115 & XTE & $9^{\prime} \times 12^{\prime}$ & $\mathrm{X}$ & $\mathrm{X}$ & & & & \\
\hline 000126 & IPN & $3^{\prime} \times 20^{\prime}$ & $\mathrm{X}$ & & & & & \\
\hline 000131 & IPN & $3.5 \times 16^{\prime}$ & $\mathrm{X}$ & & $\mathrm{x}$ & & $-1.2 \pm 0.2$ & \\
\hline 000210 & SAX & $3^{\prime}$ & $\mathrm{x}$ & $\mathrm{X}$ & & & & \\
\hline 000214 & SAX & $8^{\prime}$ & $\mathrm{X}$ & $\mathrm{X}$ & & & & \\
\hline $000301 \mathrm{~A}$ & IPN & $5^{\prime} \times 7^{\prime}$ & $\mathrm{x}$ & & & & & \\
\hline $000301 \mathrm{C}$ & $\mathrm{XTE} / \mathrm{IPN}$ & $6^{t} \times 8^{t}$ & $\mathrm{x}$ & & $\mathrm{X}$ & $\mathrm{X}$ & $-0.90 \pm 0.04$ & 2.03 \\
\hline 000307 & IPN & $6^{\prime} \times 10^{\prime}$ & $\mathrm{X}$ & & & & & \\
\hline 000315 & IPN & $4^{\prime} \times 4^{\prime}$ & $\mathrm{x}$ & & & & & \\
\hline 000323 & IPN & $50^{\prime} \times 15^{\prime}$ & $\mathrm{x}$ & & & & & \\
\hline 000326 & IPN & $12^{\prime} \times 4^{\prime}$ & $\mathrm{x}$ & & & & & \\
\hline 000408 & IPN & $24^{\prime} \times 5^{\prime}$ & $\mathrm{X}$ & & & & & \\
\hline
\end{tabular}


Table I.: GRBs localized to small error boxes. (Continued)

\begin{tabular}{|c|c|c|c|c|c|c|c|c|}
\hline Date & Method & Error & IPN & $\mathrm{XA}$ & $\mathrm{OA}$ & $\mathrm{RA}$ & Decay Index & $z$ \\
\hline 000416 & SAX & $4^{\prime}$ & & & & & & \\
\hline 000418 & IPN & $4^{\prime} \times 8^{\prime}$ & $\mathrm{X}$ & & $\mathrm{X}$ & $\mathrm{x}$ & $-1.2 \pm 0.0$ & 1.118 \\
\hline 000419 & IPN & $14^{\prime} \times 16^{\prime}$ & $\mathrm{x}$ & & & & & \\
\hline 000424 & SAX & $2 ! 5$ & & & & & & \\
\hline 000429 & IPN & $9^{\prime} \times 10^{\prime}$ & $\mathrm{X}$ & & & & & \\
\hline $000508 \mathrm{~B}$ & $\mathrm{XTE} / \mathrm{IPN}$ & $5^{\prime} \times 10^{\prime}$ & $\mathrm{X}$ & $\mathrm{X}$ & & & & \\
\hline 000516 & IPN & $2^{\prime} \times 25^{\prime}$ & $\mathrm{X}$ & & & & & \\
\hline 000519 & IPN & $10^{\prime} \times 10^{\prime}$ & $\mathrm{X}$ & & & & & \\
\hline 000528 & SAX & $4^{\prime}$ & $\mathrm{X}$ & $\mathrm{X}$ & & & & \\
\hline 000529 & SAX & $4^{\prime}$ & $\mathrm{X}$ & $\mathrm{X}$ & & & & \\
\hline 000604 & IPN & $4^{\prime} \times 8^{\prime}$ & $\mathrm{X}$ & & & & & \\
\hline 000607 & IPN & $3^{\prime} \times 10^{\prime}$ & $X$ & & & & & \\
\hline 000608 & SAX & $4^{\prime}$ & & & & & & \\
\hline 000615 & SAX & $5^{\prime}$ & & & & & & \\
\hline $000615 \mathrm{~B}$ & IPN & $3^{\prime} \times 10^{\prime}$ & $\mathrm{X}$ & & & & & \\
\hline 000616 & IPN & $5^{\prime} \times 13^{\prime}$ & $X$ & & & & & \\
\hline 000620 & SAX & $5^{\prime}$ & $\mathrm{X}$ & & & & & \\
\hline 000623 & IPN & $6^{\prime} \times 20^{\prime}$ & $\mathrm{X}$ & & & & & \\
\hline 000630 & IPN & $3^{\prime} \times 16^{\prime}$ & $X$ & & $\mathrm{X}$ & & & \\
\hline
\end{tabular}

Table I. indicates that 40 bursts were localized by the SAX/WFC, 20 were localized by the IPN alone, 7 were localized by the RXTE/PCA or RXTE/ASM, and 5 were localized by a combination of the IPN annulus and RXTE/ASM error box. Of the 72 localizations approximately $34 \mathrm{X}$-ray afterglows, 20 optical afterglows, and 13 radio afterglows have been detected. These numbers are only approximations since some afterglow identifications were not definitively associated with the GRB. Table I. also shows that the detection of an afterglow at one wavelength did not assure 
detection in any or all other wavelengths. It should be noted that rapid follow-up was not conducted for all the events listed and therefore in some cases the afterglows may have been detectable but were not observed. However in many cases followup observations provided deep upper limits at very early times. The absence of an afterglow could be the result of the GRB environment, i.e. extinction within the host galaxy, or the physics of the underlying event, i.e. a very rapidly fading counterpart. In the future deep follow-up observations at early times will help in distinguishing between these debated and fundamentally important cases (e.g., Groot et al. 1998).

On the large scale GRB afterglows are well described by the external shock model of a relativistically expanding fireball. The flux of GRB afterglows exhibits a power law decay in time, $F_{\nu} \propto t^{-\alpha}$, with indices ranging between $\alpha \sim 0.8$ and $\alpha \sim 2.3$. Despite the small number statistics currently available there appears to be two distributions of decay indices clustered around 1.2 and 2.1 with a deficiency of intermediate slopes around 1.6. GRB 990705 had a near-infrared decay slope of $\alpha=1.68$ (Masetti et al. 2000) however the fit is based on only two detections and a later upper limit falls above constant slope. These two distributions of decays may indicate a difference in circumburster medium (CBM) into which the fireball is expanding. A full review of the recent results and the fireball model is presented in Piran (1999b).

Figure 1.7 (Harrison et al. 1999) illustrates an example of a GRB optical afterglow lightcurve, GRB 990510. The break in the decay is suggestive of an increasing opening angle of an emission jet. This type of break was also observed in the afterglow lightcurve of GRB 990123. The longer wavelengths are brighter by approximately 0.5 magnitudes.

GRB 980425 has been correlated in time and space with the peculiar Type Ic supernova SN1998bw (Galama et al. 1998b). These observations provided the first solid evidence that GRBs may be associated with core collapse supernovae. However several aspects of the event make it an outlier. It has been suggested that gamma-ray bursts have a jet geometry and produce both the GRB and a core collapse supernovae. If the jet is directed towards Earth we see the GRB, even if the explosion occurs at high redshift. Supernova lightcurves may underlie all GRB afterglows and re-inspection of 
the data show week evidence for redshifted SN light curves superimposed on the late afterglow light curve (e.g., Reichart 1999; Bloom et al. 1999).

The purpose of the LOTIS experiment is to observe GRB error boxes at very early times to detect optical emission simultaneous with or shortly following the highenergy emission. This type of emission has only been observed for one unparalleled event. GRB 990123 was accompanied by a bright, $m=9.0$, prompt optical flash

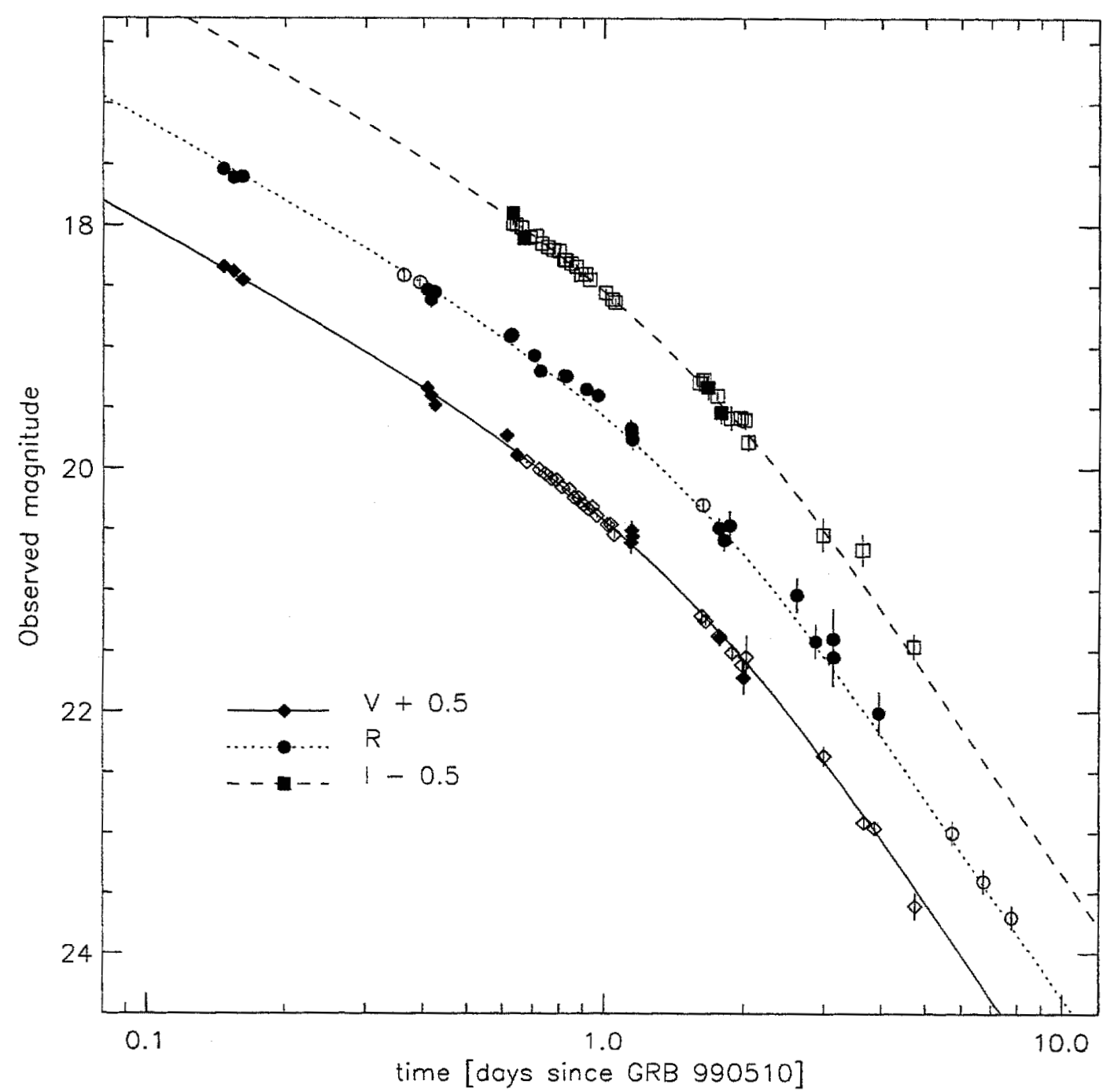

Figure 1.7 GRB 990510 multiband afterglow lightcurve (Harrison et al. 1999). 
which faded to below $m=14.0$ ten minutes after the burst (Akerlof et al. 1999). Figure 1.8 shows the optical light curve together with the gamma-ray light curve.

The peak flux of GRB 990123 in the optical band does not correlate with the peak flux in the gamma-ray band (Briggs et al. 1999a) suggesting two different emission regions (e.g., Sari \& Piran 1999a; Mészáros \& Rees 1999). This burst was a very strong event having a peak gamma-ray flux of $16.4 \gamma \mathrm{cm}^{-2} \mathrm{~s}^{-1}$ and a total fluence of $3.0 \times 10^{-4} \mathrm{erg} \mathrm{cm}^{-2}$ In addition GRB 990123 exhibited an extremely hard gamma-ray spectrum or an unusually high $E_{p}$. With only this single case of bright prompt optical emission it is difficult to determine which burst characteristics might predict such a flash. Without intermediate time observations it is unclear if the prompt emission extrapolates to the afterglow emission detected later. Measurements throughout the

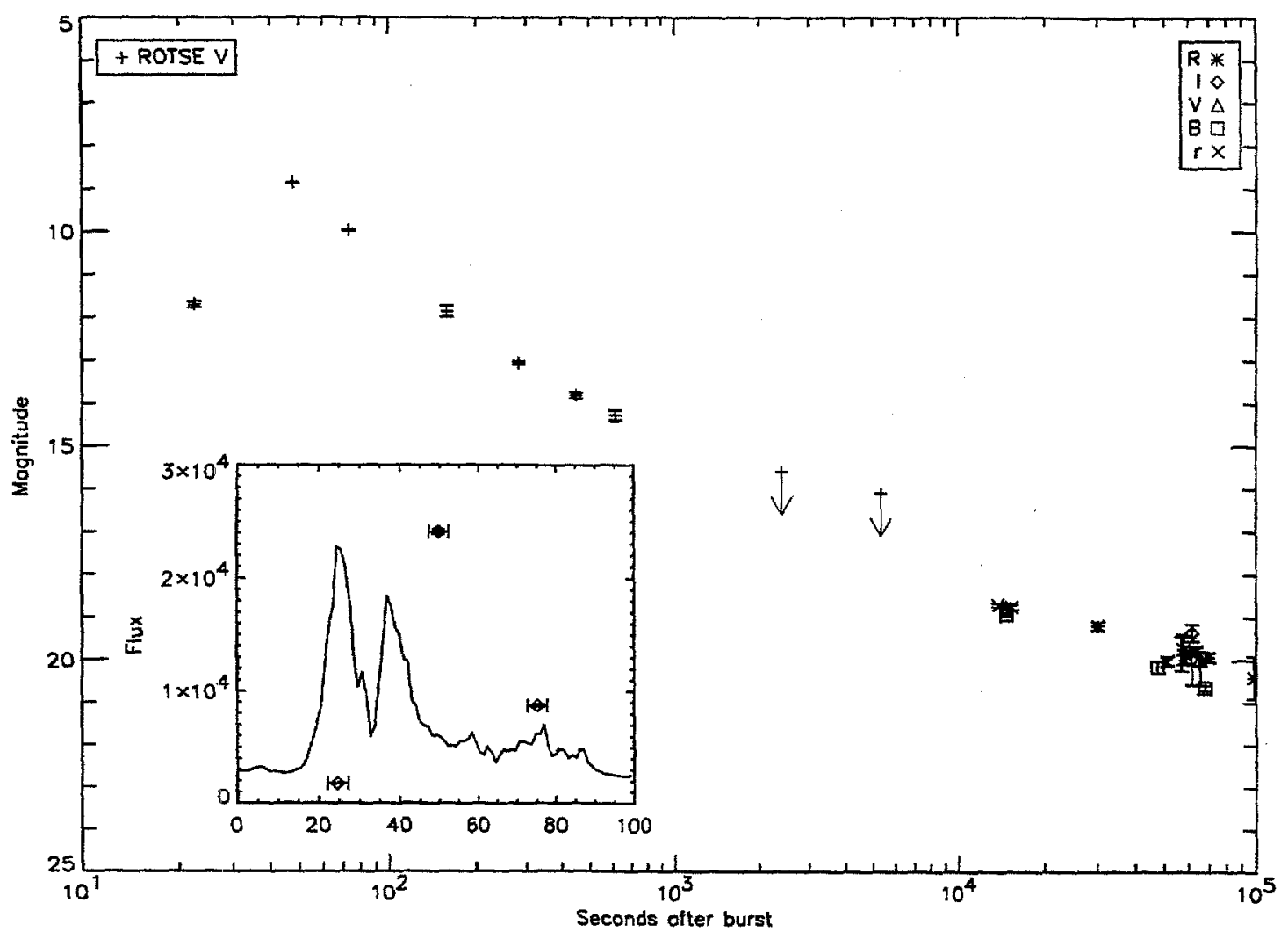

Figure 1.8 Lightcurve of GRB 990123. (Reprinted by permission from Nature Akerlof et al. 1999 copyright 1999 Macmillan Magazines Ltd.) 
afterglow duration will aid in addressing the relationship between the early afterglow and the late time data.

Now that it is clear that GRBs occur at cosmological distances it is relevant to attempt to find a relationship between burst characteristics and the redshift. Such a relationship together with the extreme luminosity of GRBs could define a standard candle by which GRBs can be used as a probe of the distant universe (Wijers et al. 1998; Hartmann et al. 1998; Totani 1999; Lamb \& Reichart 2000). Two relationships have emerged. The first relates the GRB time variability to the absolute luminosity of the burst (Fenimore \& Ramirez-Ruiz 2000) and the second relates energy dependent lags and peak luminosity in GRBs (Norris et al. 2000). Salmonson (2000) has presented a kinematic argument for the second relationship.

Finally, Figure 1.9 shows the number of GRB publications per year together with points of major discovery (Hurley 1998). During the CGRO phase there was about one publication per GRB, a truly amazing level of research activity. 


\section{NUMBER OF PUBLICATIONS PER YEAR}

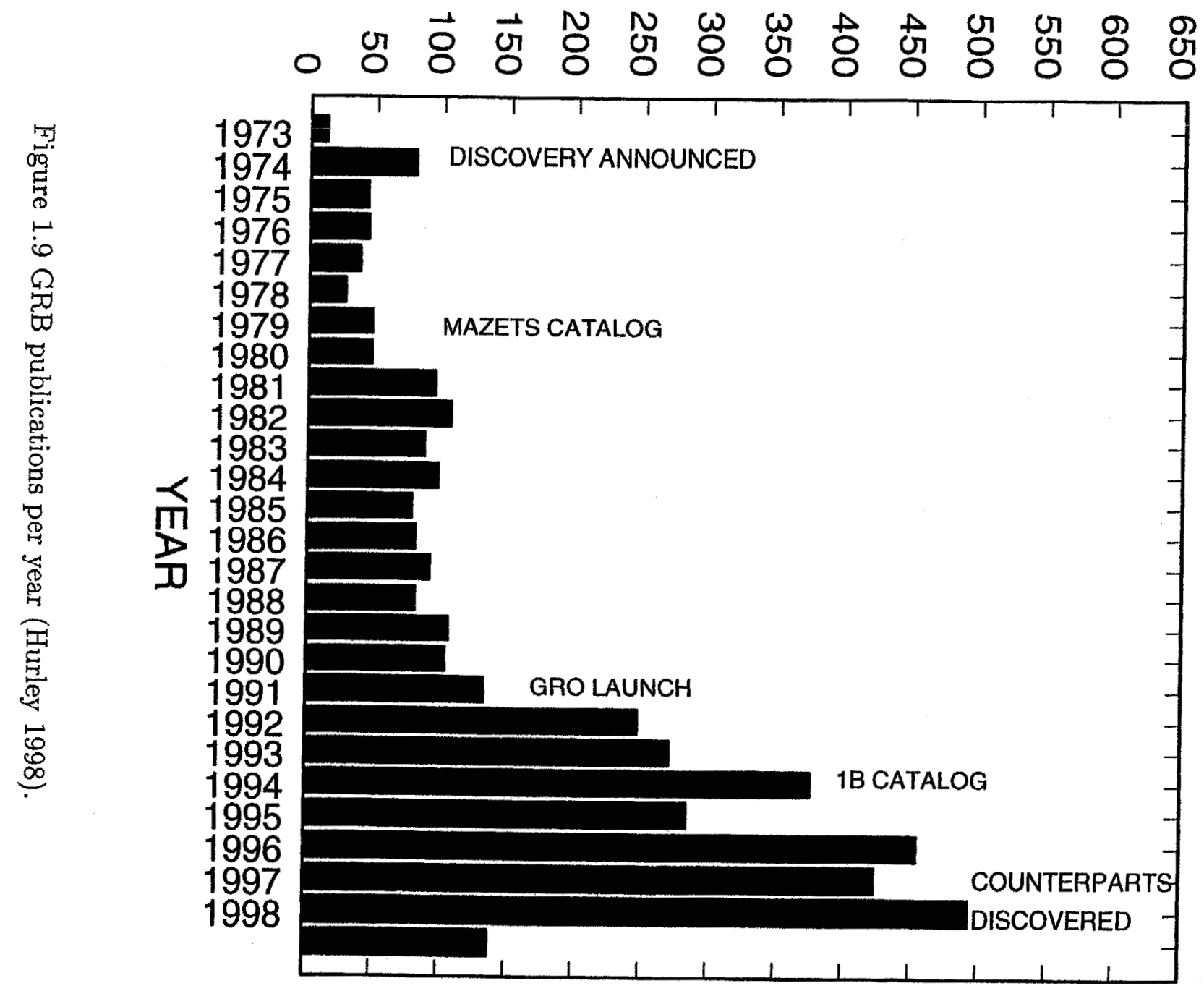




\section{CHAPTER 2}

\section{THEORY}

\section{Progenitors}

GRBs are one of the most enigmatic astrophysical phenomena. Due to the poor localizations of past gamma-ray observations no association could be made with known astrophysical sources. Until recently, the distances to GRBs were unknown leaving the of total GRB energy undetermined. Because of these limited constraints many progenitor models have been offered throughout the years.

Any GRB model must adhere to all the observational constraints:

- The rapid variability, $\delta t \sim 10^{-3} \mathrm{~s}$, in GRB light curves requires a compact source, $R<c \delta t \sim 300 \mathrm{~km}$ (non-relativistic limit).

- The event rate must be consistent with the observed rate of $\sim 1$ day $^{-1}$.

- The durations must range from short, $\mathrm{T}_{90} \sim 0.1 \mathrm{~s}$, to long $\mathrm{T}_{90} \sim 100 \mathrm{~s}$.

- The total energy must produce bursts with observed fluences of $10^{-4}-10^{-7}$ erg $\mathrm{cm}^{-2}$.

- The nonthermal power spectrum should peak in the gamma-ray regime, $E_{p} \sim$ $100 \mathrm{keV}$.

Many early models favored Galactic disk sources to avoid the enormous energy requirements for extra-galactic sources. Sources within the Milky Way must release a total energy of $E \sim 10^{37}-10^{40} \mathrm{erg}$ to account for the observed fluences. Examples of some models which have galactic disk origins include: flare induced accretion onto a compact companion such as white dwarf, neutron star, or black hole (Lamb 1973), neutron star crustal starquake (Pacini 1974), asteroid/neutron star collision (Newman \& Cox 1980), twisting and recombination of neutron star magnetic field (Liang \& Antiochos 1984), and evaporating primordial black holes (Cline \& Hong 1992). 
Even in the early days of GRB work there was evidence that the distribution might be isotropic rather than confined to the Galactic plane (e.g., Hartmann \& Epstein 1989). Therefore some theories favored origins within the Milky Way halo which keeps the energetics manageable. Examples of halo models include vibrations from a neutron star core quake undergoing phase transitions (Ramaty et al. 1980), collapse of a white dwarf into a rotating neutron star (Baan 1982), and radial oscillations of a neutron star (Muslimov \& Tsygan 1986).

Following the first results from BATSE the trend shifted towards extra-galactic models. Cosmological sources which have isotropic emission require a total energy release of $E \sim 10^{52}-10^{54}$ erg to account for the observed fluences. Many of the early models were incapable of producing this energy which would require the release of $\sim 0.01-1.0 M_{\odot} c^{2}$ of energy in the form of gamma-rays. These requirements can be reduced considerably if a jet geometry is invoked. The total energy required for a jet is $E_{\text {jet }}=4 E_{\text {iso }} / \theta^{2}$ where $E_{\text {iso }}$ is the equivalent isotropic energy and $\theta$ is the jet opening angle (Piran 1999a). The rate of cosmological events must be $\sim$ $10^{-6} \mathrm{yr}^{-1}$ per galaxy. Examples of cosmological models include shocks in Type II supernovae (Colgate 1974), binary neutron star coalescence (Eichler et al. 1989), and "failed supernovae" or collapsars (Woosley 1993; MacFadyen \& Woosley 1999). More complete overviews are given in Nemiroff (1994) and Blaes (1994).

Since the recently measured GRB redshifts indicate that they occur at large distances the large energy requirements restrict many models. Currently the most favored progenitors are the the failed supernova or collapsar, binary neutron star merger, neutron star-black hole merger, or white dwarf-neutron star merger. The final outcome of all these models is a few $M_{\odot}$ black hole surrounded by an accretion torus (see Piran 1999a). The preferred axis of the black hole-accretion-torus geometry may lead to the less energetically constraining jet. Of these models the neutron star binary merger and the collapsar have received the most attention.

Observations of binary pulsars provide evidence that binary neutron star orbits decay through emission of gravitational radiation (Taylor et al. 1979; Taylor \& Weisberg 1982). The merger time-scales for this scenario are broadly distributed but 
on average are $t_{m} \sim 10^{8} \mathrm{yr}$ (Portegies Zwart \& Spreeuw 1996) which leads to a rate of $\sim 100$ day $^{-1}$ in the Universe (Fryer et al. 1999). The high kick velocities $(\sim 400 \mathrm{~km} / \mathrm{s})$ imparted to the system during the supernova can eject them from their host galaxy before the merger occurs (Fryer et al. 1998). Therefore if binary neutron star mergers are the progenitors of GRBs they might be preferentially observed outside of their host. However the lack of a CBM might inhibit detection of the afterglow.

The collapsar model requires a high mass, $\sim 35 M_{\odot}$, main sequence star which evolves to a rapidly rotating helium star with a mass $\sim 15 M_{\odot}$. When core collapse ensues a black hole is produced promptly rather than through fall-back as is the case with normal high mass supernovae, i.e. the neutrino burst is inhibited. The rapidly rotating black hole supports an accretion disk which releases energy in the form of neutrinos through magneto-hydrodynamic (MHD) processes (e.g., Blandford \& Znajek 1977; Lee et al. 1999). The neutrinos emitted from the disk annihilate, $\nu \bar{\nu} \rightarrow e^{+} e^{-}$, in the polar regions and create the fireball jet needed to produce the GRB. The relativistic jet bores its way through the as yet uncollapsed star and breaks out as a GRB. If the jet is not energetic enough it will be "smothered" and only a peculiar SN will result (MacFadyen \& Woosley 1999).

Any event which has a luminosity much greater than that of a supernova is called a hypernova (Paczynski 1998). Therefore GRBs are hypernovae regardless of the progenitor. Collapsars and hypernovae are not interchangeable terms.

Fireballs and Relativistic Blast Waves

Regardless of the progenitor a GRB requires the deposition of a large amount of energy into a small volume on a relatively short time-scale. This scenario produces an $\mathrm{e}^{ \pm}-\gamma$-baryon fireball (Cavallo \& Rees 1978; Goodman 1986; Meszaros \& Rees 1993) The nonthermal GRB spectrum requires emission from an optically thin environment. However Goodman (1986) pointed out that a fireball is extremely optically thick to pair production, $\gamma \gamma \rightarrow \mathrm{e}^{ \pm}$. Bulk relativistic motion was suggested as a solution to this "compactness problem". The requirement for pair production by two photons of 
energies $E_{1}$ and $E_{2}$ is,

$$
E_{1} E_{2}\left(1-\cos \theta_{12}\right) \geq 2\left(m_{e} c^{2}\right)^{2}
$$

where $\theta_{12}$ is the angle between the directions of the photons, $m_{e}$ is the mass of the electron, and $c$ is the speed of light. Relativity aids in two ways. First, the observed photons are blueshifted, i.e. the source photons have energies of $1 / \Gamma$ times the observed gamma-ray photons where $\Gamma$ is the bulk Lorentz factor. Second, the relativistic beaming reduces the angle between the photons allowing $E_{1} E_{2}$ to become large before pair production is allowed (Krolik \& Pier 1991). Observations of high energy photons from GRBs requires bulk Lorentz factors of $\Gamma \gtrsim 100$ (Baring \& Harding 1997).

Shemi \& Piran (1990) showed that most of the fireball radiation energy will be converted to kinetic energy of matter even for very low baryon contamination. A method was necessary to re-extract the energy from the matter to produce the observed GRBs. Rees \& Meszaros provided a method through shocks, either external (1992) or internal (1994).

\section{Internal Shocks and GRBs}

There are two methods by which the central engine of a GRB can deposit its energy; impulsively or through an unsteady wind. The impulsive deposition produces a single relativistically expanding shell. This shell returns its kinetic energy to photons through interaction with the CBM which produces an external shock (Meszaros \& Rees 1993). The unsteady wind deposits energy over the duration of the burst producing many relativistically expanding shells with different bulk Lorentz factors (Rees \& Meszaros 1994). As faster shells catch up to slower shells they collide producing relativistic internal shocks. Eventually the shells have merged into a single or a few shells which interact with the CBM again producing external shocks.

The first suggested mechanism of extracting the energy from a relativistic blast wave was through radiation produced in external shocks (Rees \& Meszaros 1992). This mechanism required bulk Lorentz factors of $\Gamma=10^{2}-10^{3}$. It was found that less extreme values of $\Gamma \sim 10^{2}$ were required if GRBs result from internal shocks 
(Rees \& Meszaros 1994). One additional problem with external shocks is that they are less likely than internal shocks to reproduce the observed time structure of GRBs (Fenimore et al. 1996; Sari \& Piran 1997). However, Dar (1998) argues against the capability of internal shocks to reproduce the observed GRB time variability.

\section{External Forward Shocks and Afterglows}

The favored origin of the long-wavelength afterglow of GRBs is synchrotron emission of electrons accelerated to a power law distribution in an enhanced magnetic field. Following Sari et al. (1998) we assume the relativistic shock is propagating through a uniform CBM of particle density $n$. The spectrum of the resultant emission is characterized by three break frequencies: the characteristic synchrotron frequency $\nu_{m}$, which is the frequency at which the bulk of the electrons are emitting, the cooling frequency $\nu_{c}$, which is the frequency above which the electrons are loosing energy hydrodynamically faster than they can contribute to the spectrum, and the synchrotron self absorption frequency $\nu_{a}$, which is the frequency below which the electron distribution is absorbing the emitted radiation. The resultant shape of the spectrum depends on whether the cooling frequency is below or above the characteristic synchrotron frequency. These two cases are referred to as the fast cooling and slow cooling regimes, respectively.

Figure 2.1 shows the synchrotron spectra for both the fast cooling and slow cooling regimes (Sari et al. 1998). For the case of fast cooling electrons the spectrum has a shape described by,

$$
F_{\nu}= \begin{cases}\left(\nu / \nu_{c}\right)^{1 / 3} F_{\nu, \max }, & \nu_{c}>\nu \\ \left(\nu / \nu_{c}\right)^{-1 / 2} F_{\nu, \max }, & \nu_{m}>\nu>\nu_{c} \\ \left(\nu_{m} / \nu_{c}\right)^{-1 / 2}\left(\nu / \nu_{m}\right)^{-p / 2} F_{\nu, \max }, & \nu>\nu_{m}\end{cases}
$$


For the slow cooling electrons the spectrum is described by,

$$
F_{\nu}= \begin{cases}\left(\nu / \nu_{m}\right)^{1 / 3} F_{\nu, \max }, & \nu_{m}>\nu, \\ \left(\nu / \nu_{m}\right)^{-(p-1) / 2} F_{\nu, \max }, & \nu_{c}>\nu>\nu_{m}, \\ \left(\nu_{c} / \nu_{m}\right)^{-(p-1) / 2}\left(\nu / \nu_{c}\right)^{-p / 2} F_{\nu, \max }, & \nu>\nu_{c} .\end{cases}
$$

where $F_{\nu, \max }$ is the peak flux and $p$ is the index of the electron power-law distribution.

The spectral shape does not depend on the hydrodynamical evolution of the shock however the break frequencies and the peak fiux do. Sari et al. (1998) consider two extreme cases of hydrodynamical evolution, fully radiative or fully adiabatic.

The values of the break frequencies and the peak flux can be estimated from five blast wave parameters and two observable parameters. The important blast wave parameters are: $E_{52}$ the total energy in the shock in units of $10^{52} \mathrm{erg}, \epsilon_{B}$ and $\epsilon_{e}$ the equipartition fraction of the blast wave energy which goes into the magnetic field and the fraction which goes into the electrons, $\gamma_{0}$ the initial Lorentz factor of the shell, and $n_{1}$ the number density of the CBM in units of $\mathrm{cm}^{-3}$. The two observable parameters are $t_{d}$ the observation time in days after the burst and $D_{28}$ the distance to the burst in units of $10^{28} \mathrm{~cm}$.

For the case of fully adiabatic evolution the break frequencies and the peak flux are,

$$
\begin{gathered}
\nu_{c}=2.7 \times 10^{12} \epsilon_{B}^{-3 / 2} E_{52}{ }^{-1 / 2} n_{1}{ }^{-1} t_{d}^{-1 / 2} \mathrm{~Hz}, \\
\nu_{m}=5.7 \times 10^{14} \epsilon_{B}^{1 / 2} \epsilon_{e}^{2} E_{52}{ }^{1 / 2} t_{d}^{-3 / 2} \mathrm{~Hz} \\
F_{\nu, \text { max }}=1.1 \times 10^{5} \epsilon_{B}^{1 / 2} E_{52} n_{1}{ }^{1 / 2} D_{28}{ }^{-2} \mu \mathrm{Jy} .
\end{gathered}
$$

For the fully radiative case the break frequencies and the peak flux are given by,

$$
\begin{aligned}
& \nu_{c}=1.3 \times 10^{13} \epsilon_{B}^{-3 / 2} E_{52}{ }^{-4 / 7} \gamma_{2}{ }^{4 / 7} n_{1}{ }^{-13 / 14} t_{d}{ }^{-2 / 7} \mathrm{~Hz} \\
& \nu_{m}=1.2 \times 10^{14} \epsilon_{B}^{1 / 2} \epsilon_{e}^{2} E_{52}{ }^{4 / 7} \gamma_{2}^{-4 / 7} n_{1}{ }^{-1 / 14} t_{d}{ }^{-12 / 7} \mathrm{~Hz}
\end{aligned}
$$




$$
F_{\nu, \max }=4.5 \times 10^{3} \epsilon_{B}^{1 / 2} E_{52}^{8 / 7} \gamma_{2}^{-8 / 7} n_{1}^{5 / 14} D_{28}^{-2} t_{d}^{-3 / 7} \mu \mathrm{Jy}
$$

For both the adiabatic and radiative cases $\nu_{m}$ decreases in time faster than $\nu_{c}$. For typical blast wave parameters the electrons are in the fast cooling regime early and later evolve to the slow cooling case, i.e. $\nu_{c}<\nu_{m}$ initially. The transition between fast cooling and slow cooling occurs when $\nu_{c}=\nu_{m}$ at $t_{0}$,

$$
t_{0}= \begin{cases}210 \epsilon_{B}^{2} \epsilon_{e}^{2} E_{52} n_{1} \text { days, } & \text { adiabatic } \\ 4.6 \epsilon_{B}^{7 / 5} \epsilon_{e}^{7 / 5} E_{52}^{4 / 5} \gamma_{2}^{-4 / 5} n_{1}^{3 / 5} \text { days, } & \text { radiative. }\end{cases}
$$

\section{External Reverse Shocks and Prompt Emission}

Prior to the detection of prompt optical emission from GRB 990123, Sari \& Piran (1999b) predicted an early optical flash resulting from synchrotron emission in the reverse external shock. The physics of the reverse shock is the same as for the forward shock but some of the parameters are different. Sari \& Piran (1999b) argue that the magnetic field and Lorentz factor are the same in the reverse and forward shocks. Therefore the cooling frequency is the same in both shocks.

The characteristic synchrotron frequency however depends on the random Lorentz factor of the electrons. In the forward shock the random Lorentz factor at time $t_{A}$, when the reverse emission peaks, is $\gamma_{A}$. In the reverse shock the random Lorentz factor is $\gamma_{0} / \gamma_{A}$ where $\gamma_{0}$ is the initial Lorentz factor. Therefore the characteristic frequency is much lower in the reverse shock than in the forward shock.

The break frequencies in the reverse shock at the peak of the emission are given by,

$$
\nu_{c}=8.8 \times 10^{15}\left(\frac{\epsilon_{B}}{0.1}\right)^{-3 / 2} E_{52}^{-1 / 2} n_{1}^{-1} t_{A}^{-1 / 2} \mathrm{~Hz}
$$

and

$$
\nu_{m}=1.2 \times 10^{14}\left(\frac{\epsilon_{e}}{0.1}\right)^{2}\left(\frac{\epsilon_{B}}{0.1}\right)^{1 / 2}\left(\frac{\gamma_{0}}{300}\right)^{2} n_{1}^{1 / 2} \mathrm{~Hz}
$$

Application of the reverse shock synchrotron model is given in the Data Analysis and Interpretation (Chap. 5). The scenario for emission from fireball blast waves presented here is depicted schematically in Figure 2.2. 

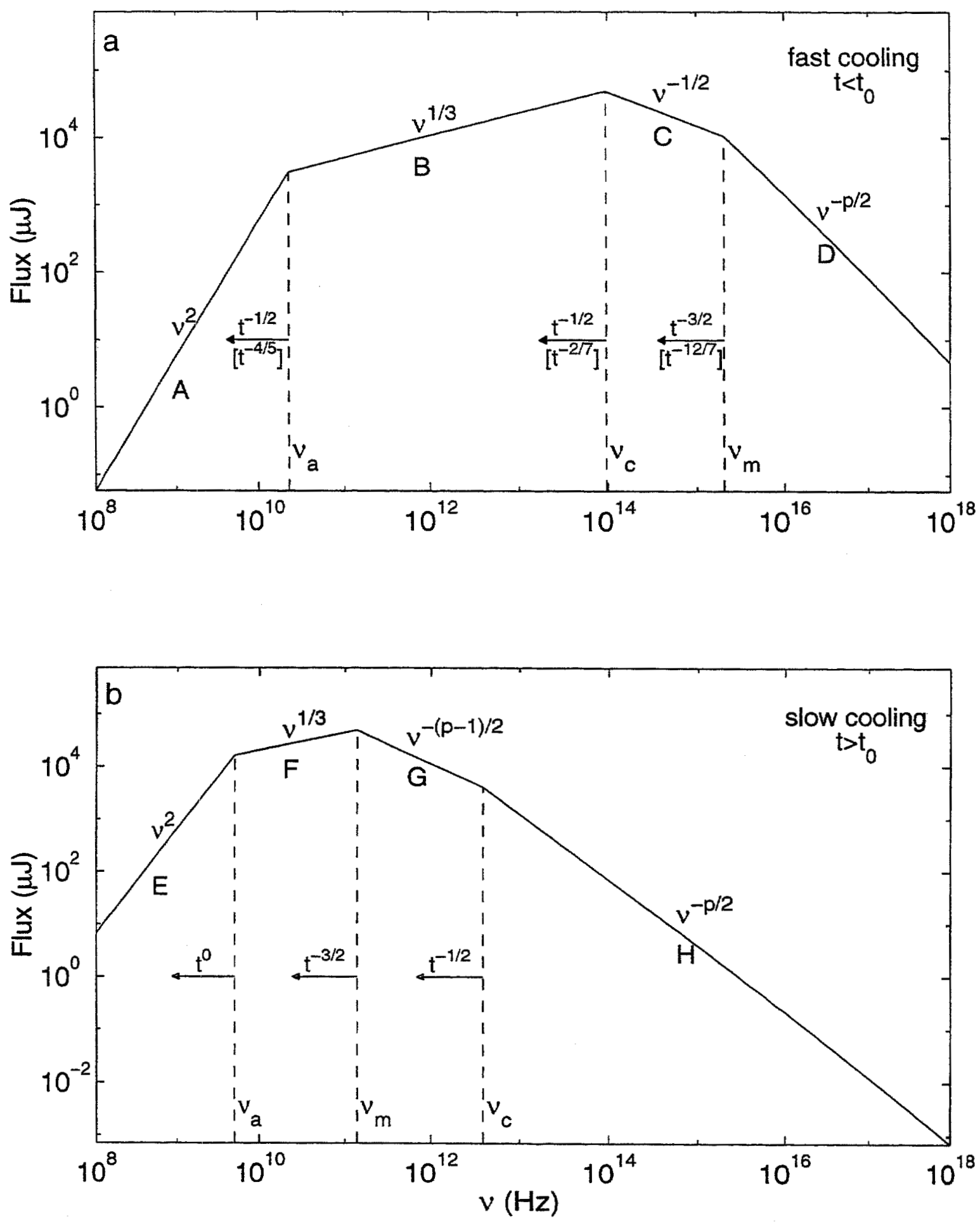

Figure 2.1 Synchrotron spectrum for the cases of fast and slow cooling electrons (Sari et al. 1998). 


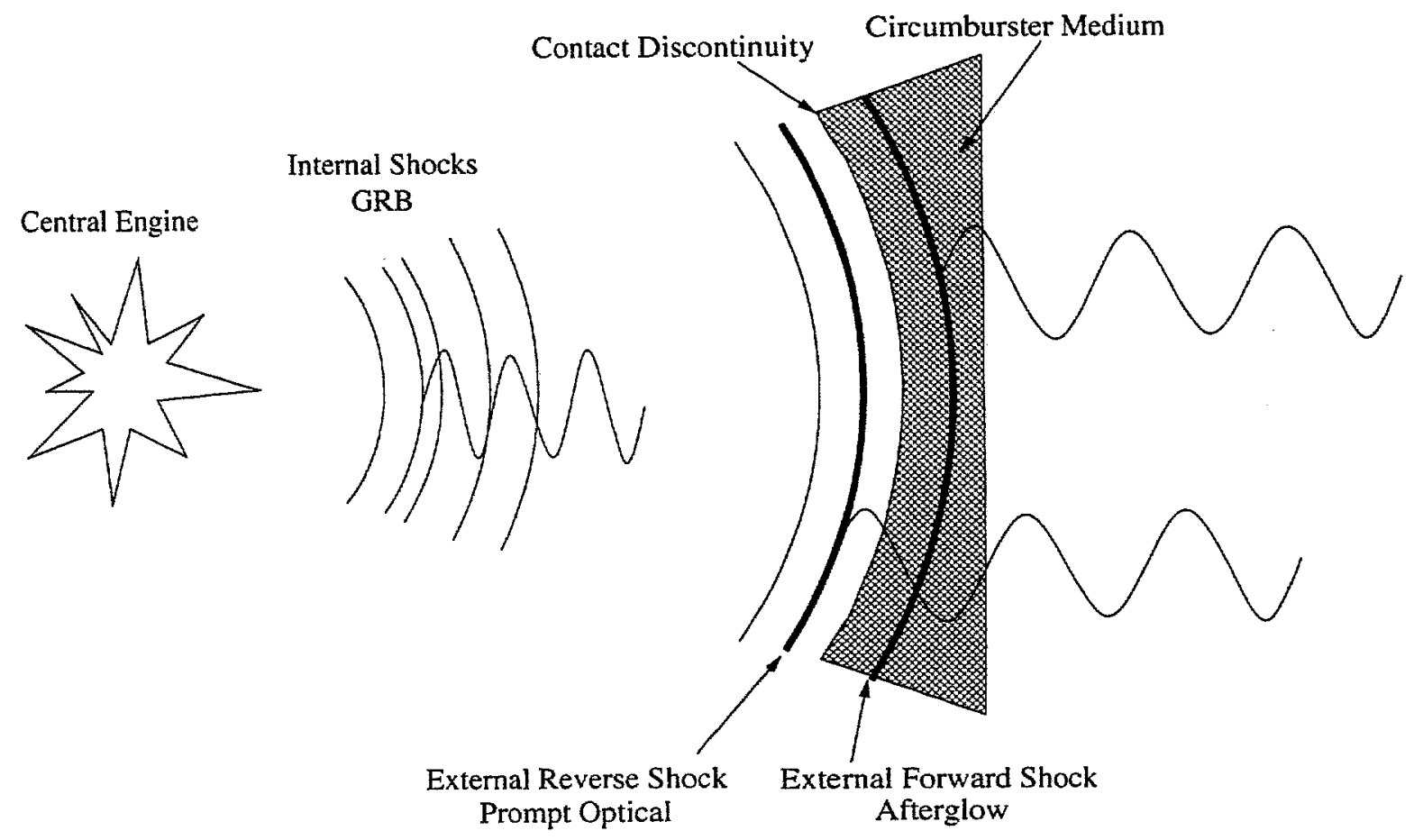

Figure 2.2 Relativistic blast wave schematic. 


\section{CHAPTER 3}

\section{EXPERIMENTAL SETUP}

\section{System Requirements}

Any system dedicated to the search for simultaneous optical counterparts of GRBs must adhere to several unique design requirements. A large field-of-view is necessary to maximize the probability of covering an entire $3 \sigma$ BATSE error circle $\left(5^{\circ}-\right.$ $20^{\circ}$ Original Trigger; see 4.3). A sensitive detection threshold is required to maximize the probability of detecting the (possibly) faint optical emission from a GRB or to place meaningful constraints on GRB models in the event of a non-detection. A completely automated system is needed in order to respond as rapidly as possible to a GRB trigger and thus maximize the probability of imaging simultaneous with gamma-ray emission. A review of the LOTIS hardware is presented in Park et al. (1998a).

\section{LOTIS Hardware}

To maximize the field-of-view the LOTIS telescope utilizes short focal length optics together with a large format CCD. Ideally a large aperture would be used to increase the detection threshold but a compromise must be made to allow rapid pointing and to ensure a reasonable cost. A commercially available Canon EF 200 $\mathrm{mm} \mathrm{f} / 1.8 \mathrm{~L}$ lens was chosen for the system because it provided the highest quality optics at a minimum cost. Each lens has an effective aperture of $11.1 \mathrm{~cm}(4.37 \mathrm{in})$ with a unique multi-element design, shown in Figure 3.2, which corrects for both comatic and chromatic aberration.

The imaging sensor used in the LOTIS camera is a Loral Fairchild 4432048 x 2048 pixel CCD. These CCDs, shown in a cut-away digram in Figure 3.3, have a pixel size of $15 \mu \mathrm{m} \times 15 \mu \mathrm{m}$ and provide a focal plane area of approximately $3.10 \mathrm{~cm}$ $\mathrm{x} 3.10 \mathrm{~cm}$. The single axis field-of-view is given by

$$
\mathrm{FOV}=2 \times \arctan \left(\frac{1 / 2 \times L}{f}\right)=2 \times \arctan \left(\frac{1 / 2 \times 31.0)}{200.0}\right) \approx 8.86,
$$




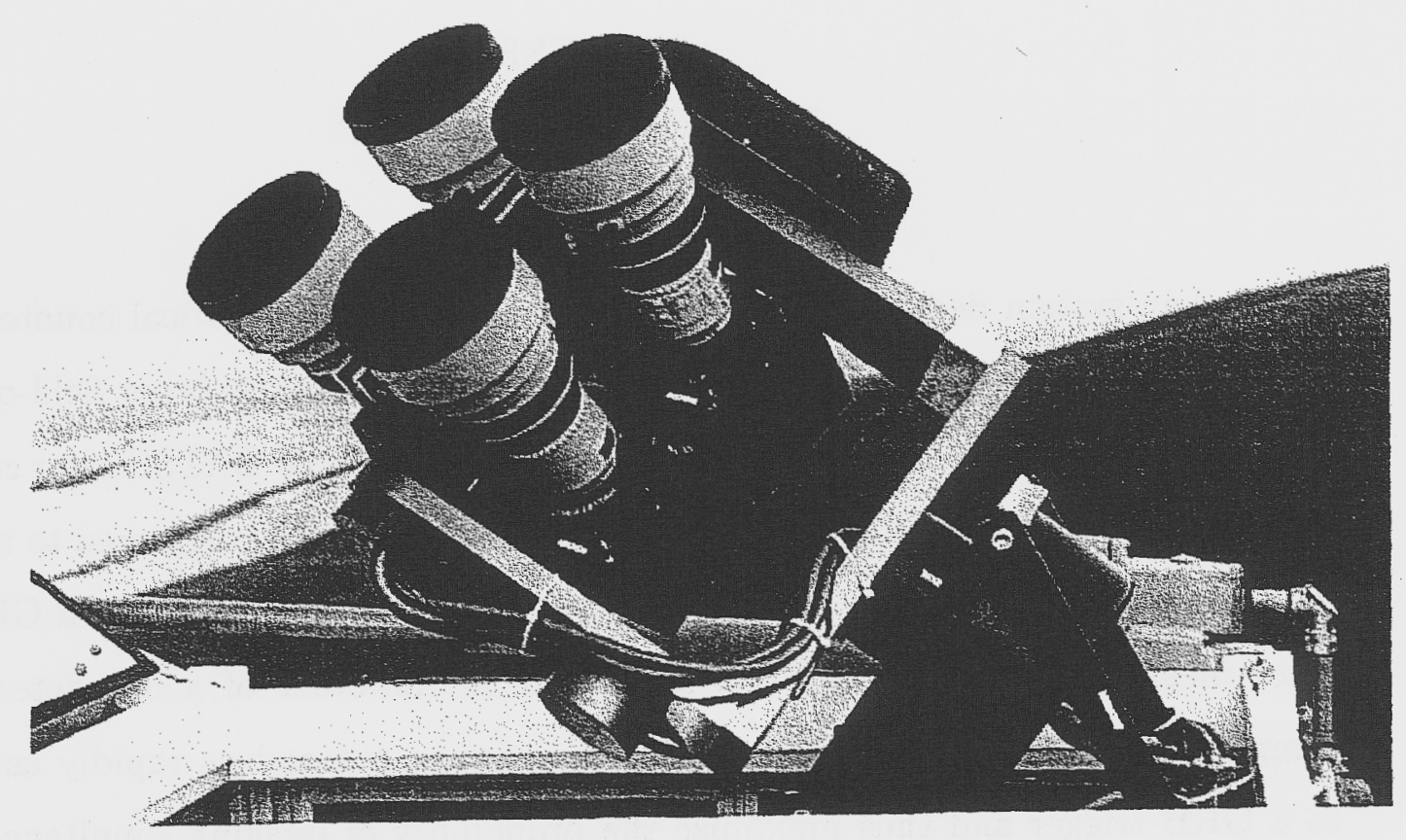

Figure 3.1 The LOTIS telescope.

or a total field-of-view of $8.86 \times 8: 86$.

Even this large field-of-view is insufficient for covering the $3 \sigma$ BATSE error boxes. Therefore the LOTIS telescope consists of a $2 \times 2$ array of the lens/camera assemblies. These assemblies are mounted on aluminum plates which offset the pointing by approximately 4.25 in the right ascension direction and 4.25 in the declination direction. This provides an overlap of approximately $20^{\prime}$ in both right ascension and declination and provides a total field-of-view for the telescope of $17.4 \times 1704$.

Prior to May 1998 LOTIS was equipped with the Loral 442A CCD which was similar in format to the $443 \mathrm{CCD}$ with no thermoelectric cooling. In this configuration the LOTIS sensitivity was constrained by a large dark current since the uncooled CCDs operated with a focal plane temperature near $30^{\circ} \mathrm{C}$.

In order to increase the sensitivity the dark current must be kept to a minimum by cooling the CCD. As shown in Figure 3.3 the Loral 443 CCDs are equipped with built in thermoelectric cooling. A water cooled copper cooling block has been 
implemented behind the CCD to remove the heat from the backside. The cooling block is supplied with an antifreeze-water mixture at a temperature of $-10^{\circ} \mathrm{C}$. The $\Delta T$ across the CCD at a TE current of $1 \mathrm{amp}$ is approximately $10^{\circ} \mathrm{C}$ and therefore the focal plane array temperature is kept at $0^{\circ} \mathrm{C}$. Although the $\mathrm{CCD}$ enclosure is backfilled with dry nitrogen condensation collected on the outside of the CCD window when the TEs were active. Therefore the CCD and CCD board were enclosed in a dry nitrogen flushed enclosed chamber.

The readout electronics were designed and fabricated at LLNL. Figure 3.4 shows a block diagram of the LOTIS camera readout electronics. In addition to the CCD board each camera enclosure houses an Analog-to-Digital Converter (ADC) board and a computer control board.

The CCD board consists of a socket for the CCD, clock drive circuitry, the first stage signal amplifier/buffer, and a focal-plane-array (FPA) temperature monitor. The clock drive voltages are regulated with levels that are resistor programmable. The amplifier gain value was selected to obtain the optimum desired sensitivity for

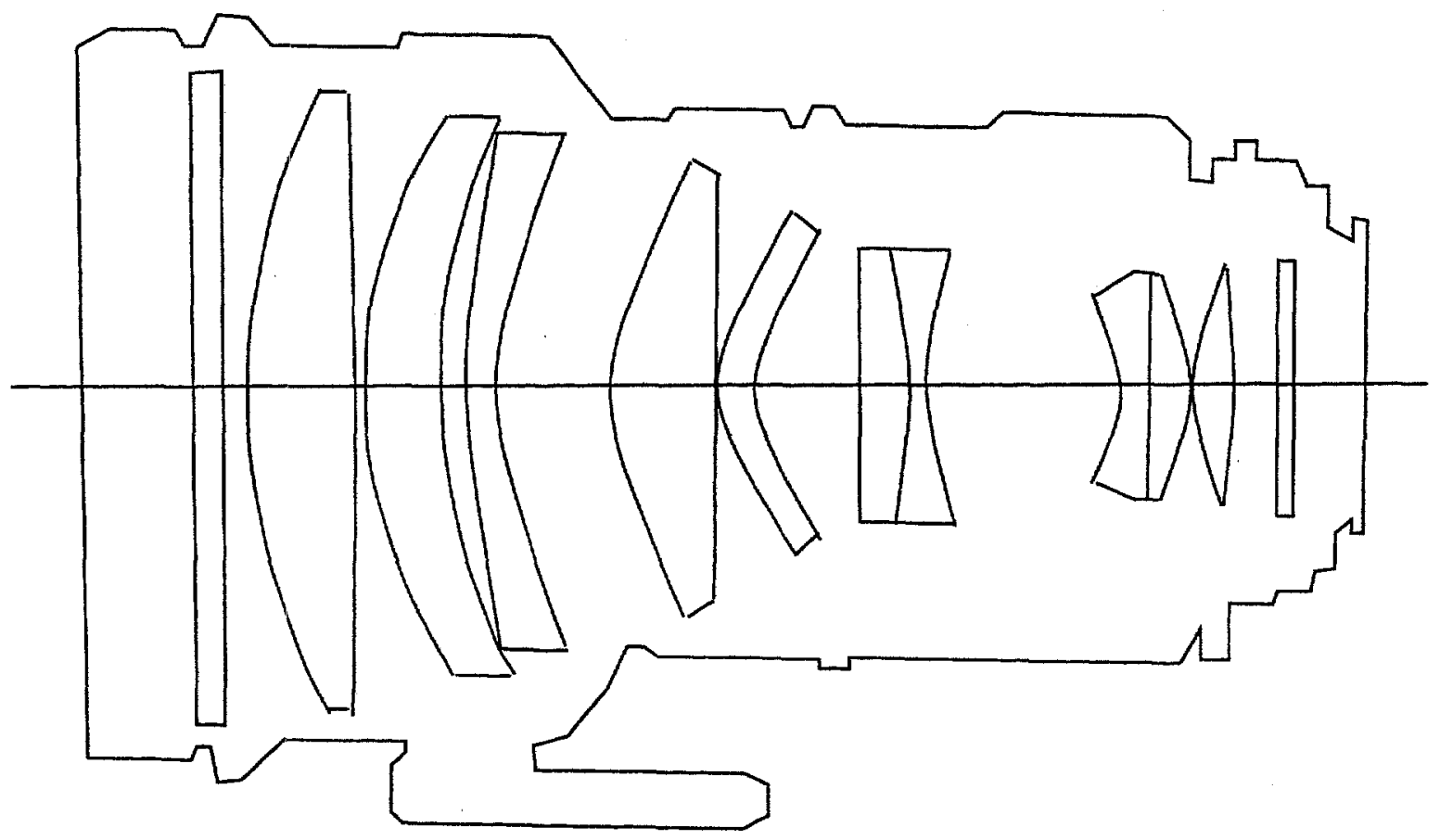

Figure 3.2 Optical design of the LOTIS Canon EF 200mm Lens. 
the CCD. The amplifier provides a low impedance drive of the CCD signal which is routed to the ADC board.

The ADC board provides the second stage signal amplification, Correlated Double Sampling (CDS) circuitry, and analog-to-digital conversion. The 12 bit ADC sampling rate is fixed at $500 \mathrm{k}$ samples/sec as a compromise between low read noise
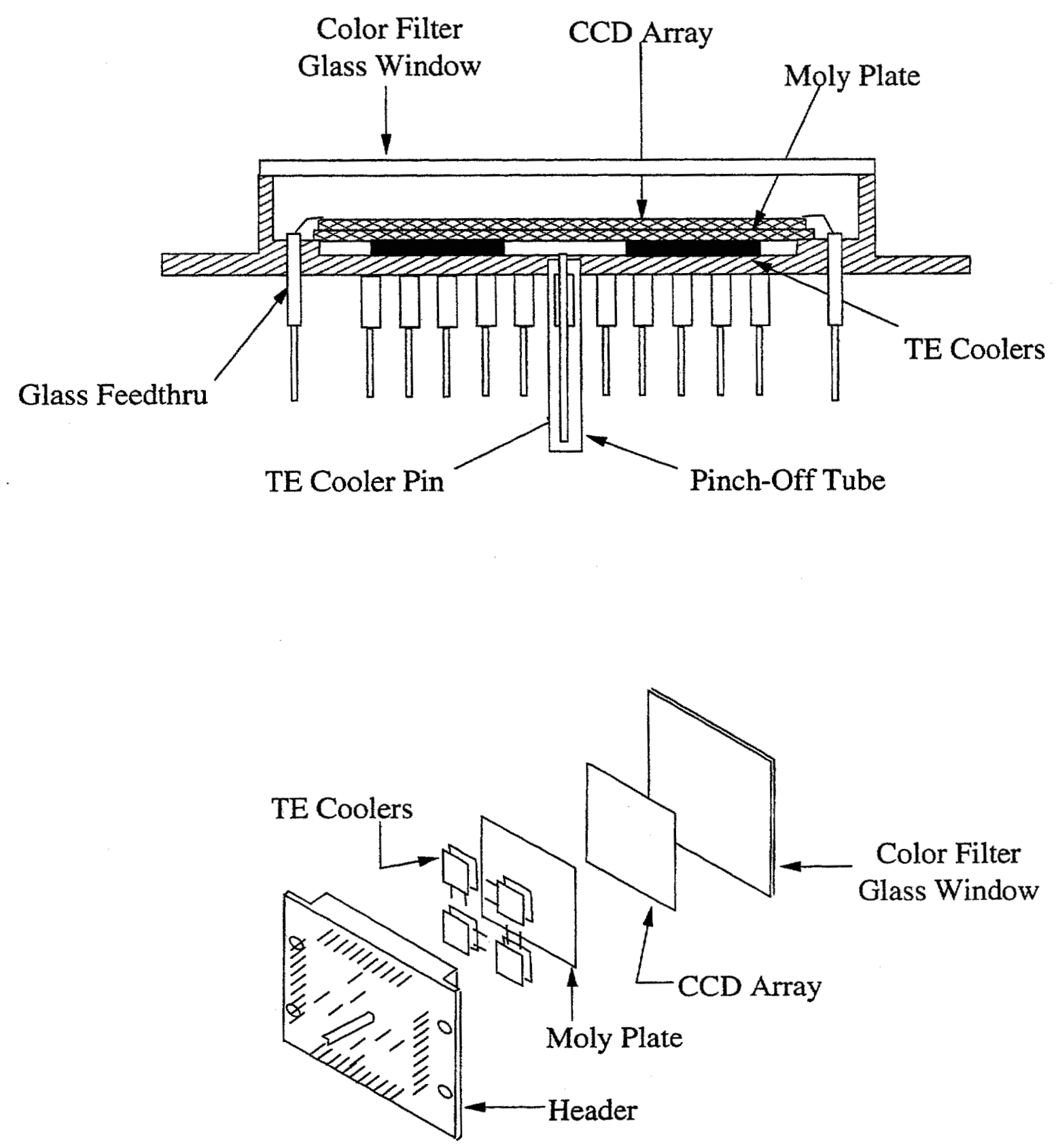

Figure 3.3 Cut away diagram of the Loral 443 CCD. 


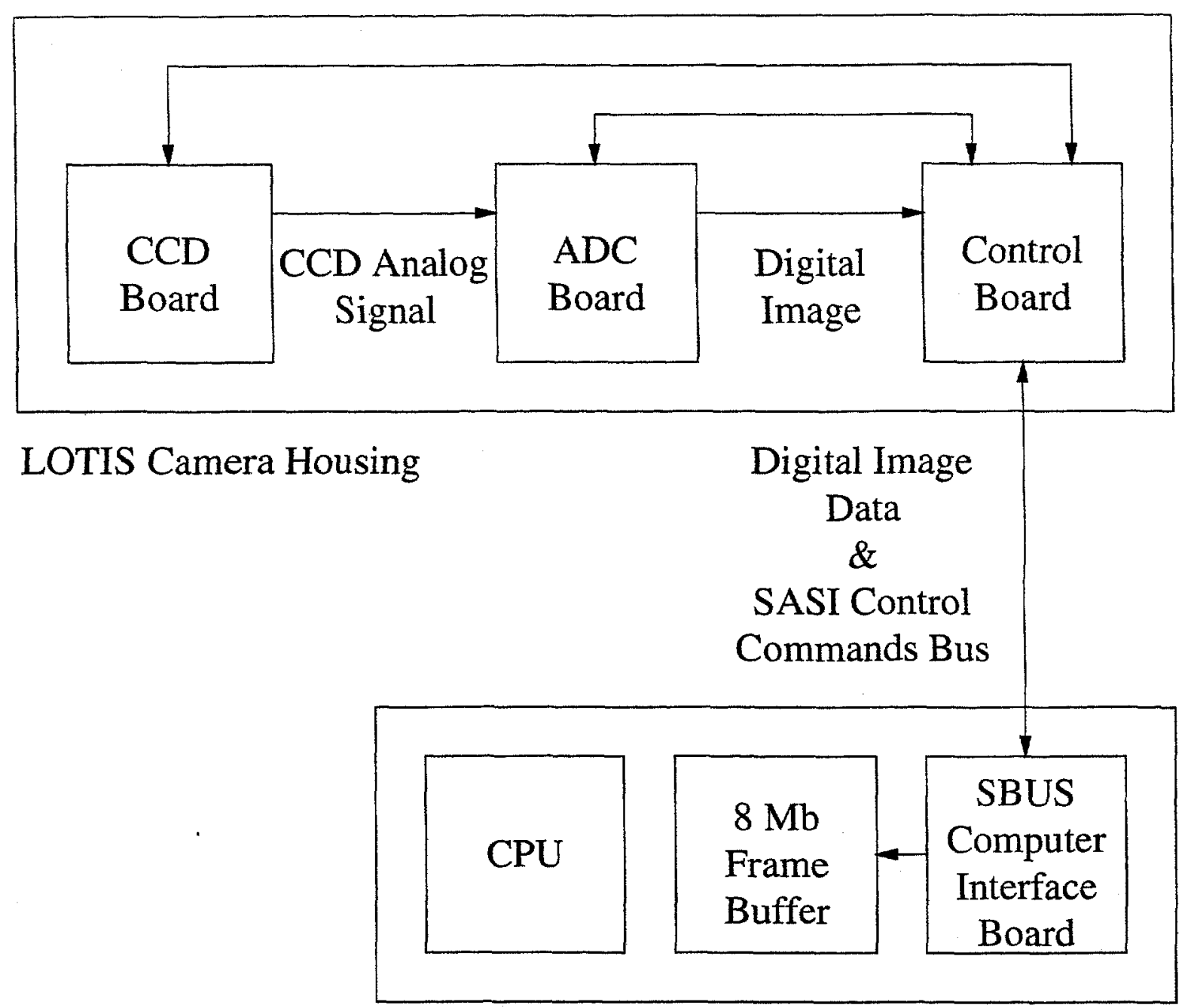

Sun/Sparc2 Camera Computer

Figure 3.4 LOTIS camera readout electronics design.

operation and CCD readout speed. This sampling rate results in $\mathrm{a} \sim 75 \mathrm{e}^{-}$read noise and an 8 second readout time. The system gain results in approximately 20 electrons per analog to digital units (ADUs; grey levels). The parallel digital output of the ADC is buffered and routed to the computer control board.

The computer control board provides all the control signals for the CCD and ADC boards. The control board accepts a suite of commands from the camera computer and returns status information via a modified synchronous addressable serial interface (SASI). The SASI protocol is an 8-bit serial bus modified to accommodate 
16-bit command/status words. The control board receives the parallel image data from the ADC board, serializes it, and sends it to the host computer over a separate bus at a rate of $6 \mathrm{Mbit} / \mathrm{s}$. No portion of the image data is stored in the camera.

The mating interface from the camera control board to the camera computer is a custom SBUS card equipped with programmable logic devices (PLDs). A pair of Altera PLDs are programmed to accommodate the user specific commands and control functions. The computer controllable functions include setting the integration time, data transfer enable/disable, video enable/disable, FPA temperature monitor, bias level or offset, shutter control, fast flush, and test pattern generation.

The camera enclosure measures approximately 6" square. The housing is equipped with electrical connectors, a cooling fan, and a shutter mount. The shutter is a commercially available Melles Griot $53 \mathrm{~mm}$ aperture mechanical device. The front face of the enclosure is fitted with three 100 turn/inch spring loaded screws. Receptacles in the CCD board accept the screws and allow for tip/tilt adjustment of the CCD.

The LOTIS system is fixed to a rapidly slewing equatorial mount manufactured by Epoch Instruments. The LOTIS mount can point payloads of up to $50 \mathrm{lbs}$ to any part of the sky in approximately $4 \mathrm{~s}$. The system has an absolute pointing accuracy of $\sim 0.01^{\circ}$ and a tracking stability of $\sim 15^{\prime \prime}$ per minute.

LOTIS is housed in an automated clamshell enclosure which permits sky coverage down to $20^{\circ}$ elevation. A Duff-Norton electro-mechanical actuator (load rated at $1500 \mathrm{lbs}$ ) opens and closes the roof. A rack mount in the lower half of the housing carries the mount controller, camera power supplies, and an Allen-Bradley industrial controller. The water chiller and a small air conditioning unit also reside in the clamshell.

The LOTIS control room which houses the computers, UPS, and tape drives is located approximately $40 \mathrm{ft}$ from the telescope. The cables from the cameras to the clam shell run in an underground conduit.

Figure 3.8 shows a block diagram of the LOTIS data acquisition system. This system includes many features required for reliable remote automated operation. In 
addition to the computer controllable mount and electro-optical sensors, the system includes a computer controllable housing, diagnostic sensors, and a UPS to handle power failures.

The LOTIS system is fully automated. Each night the on-line software is scheduled to start at a specific time. The automated process opens the telescope housing, initializes the hardware, establishes connections to the communication code and the camera control codes, obtains calibration data, begins a full-sky survey, and responds to GRB triggers. Prior to sunrise the process parks the telescope, closes the housing, powers down the system, analyzes diagnostic data, archives the data, and sends out an observing report via e-mail. The system monitors the weather and shuts down if rain is detected.

The LOTIS online software has three major components: the communication link to GCN, the telescope management code, and the camera control and image acquisition code. The computer system consists of a network of five SUN Sparc workstations. A Sparc 10 host computer controls the supervisory functions while four Sparc 2s operate each of the four cameras.

The communication link and the telescope control software run on the host computer. The communication link code has an internet socket connection to the GCN distribution computer at NASA/Goddard Space Flight Center. This link is maintained continuously to avoid delays in establishing a link. GCN packets are sent at one minute intervals and the LOTIS system echoes the packet back to GCN. If a break-in-connection is detected by either system the codes automatically attempt to re-establish a connection. When the communications link receives a GRB trigger a Unix socket packet is sent to the telescope manager code. If LOTIS is in observation mode and the coordinates are viewable the telescope manager sends a fast flush signal to the CCDs and moves the telescope. GRB disk directories and FITS headers are created. Once the telescope is in position data acquisition begins. All images are stored directly to disk. 


\section{Super-LOTIS Hardware}

In the past LOTIS relied on imprecise GRB coordinates provided by BATSE. More recently precise localizations have been realized through $\mathrm{x}$-ray detection (SAX and RXTE) and IPN triangulation. The smaller error boxes provided by these methods warrant the use of a more sensitive smaller field-of-view system to search for prompt optical emission and the early-time afterglow. In addition, future satellite missions, such as HETE-2 and Swift, promise many more detections localized to small error boxes in real-time. The next generation LOTIS system, Super-LOTIS, was designed to take full advantage of smaller error boxes. An additional design requirement came directly from the LOTIS results. The lack of bright prompt optical counterparts and early time afterglow requires a system with greater sensitivity.

In the very near future SAX, HETE-2 and RXTE are only expected to produce approximately two accurate localizations per month. This is far fewer than the number of bursts detected by BATSE. Therefore in addition to covering the small error boxes the Super-LOTIS telescope needed the capability of covering the BATSE error boxes at very early times. For this reason a compromise was made between sensitivity and large field-of-view.

The Super-LOTIS telescope is a $60 \mathrm{~cm}$ parabolic reflector mounted on a Boller $\&$ Chivens German equatorial mount (Figure 3.9). The primary mirror has an f-ratio of $\mathrm{f} / 3.5$. To provide a large field-of-view the Super-LOTIS camera is mounted at the prime focus. This fast system results in significant coma and thus requires the additional optics of a coma corrector assembly. Figure 3.10 shows the 4-element coma corrector custom designed for the Super-LOTIS telescope. The coma corrector provides a point-spread-function (psf) at the edge of the field which has a width confined to a single pixel (1.5" pixel; psf).

The Super-LOTIS camera is a modified LOTIS camera. All components are the same with the exception of an added stage of $\mathrm{TE}$ cooling. To provide standard BVR photometry the Super-LOTIS telescope will also be equipped with a filter "wheel" assembly. The filter wheel is not yet implemented. 
Super-LOTIS recently began routine operations from a permanent site at Steward Observatory's Kitt Peak Station. In the year prior to the relocation the system operated from the grounds of LLNL (March 1999 - June 2000). During much of this time the system was performing engineering and debug runs. In addition to the bright surroundings the system often was not operating at full capacity.

\section{Camera Calibration}

Each camera was focused in the lab using an off-axis collimator with a pin hole which had an angular image which was much smaller than the pixel size. A $100 \mu \mathrm{m}$ diameter pinhole was used at the $5 \mathrm{~m}$ focal length of the collimator. The fast lens results in a focal depth of $\sim 120 \mu \mathrm{m}$ per half turn of the screw. The focus screws were adjusted to minimize the image of the pinhole in all four corners and the center of the CCD. At each CCD location the image was fit to a Gaussian and the width was used as a figure of merit in producing a good focus across the FPA. Typical Gaussian fits had a $1 \sigma$ width of 0.4 pixels. This procedure is repeated in the field as necessary using the width of the stellar point spread function.

Prior to TE cooling the LOTIS CCDs had a dark current of $\sim 800 e^{-} / \mathrm{sec} / \mathrm{pixel}$. After TE cooling was implemented the dark current dropped to $\sim 50 e^{-} / \mathrm{sec} / \mathrm{pixel}$. Figure 3.12 shows measurements of the dark current for the Loral 442A CCD. The bar at $25^{\circ} \mathrm{C}$ is the manufacturer specification.

\section{Observation Strategy}

\section{Sky Patrol}

Each clear night when LOTIS is not responding to a GRB trigger the system carries out a routine sky patrol. With the LOTIS field-of-view and a $30 \mathrm{~s}$ (10 s) integration time LOTIS can cover the sky 2 (4) times per night. This full sky coverage takes about 105 telescope pointings. During each individual sky patrol two images are obtained at each pointing. (state clearer). Each camera is equipped with a 9 gigabyte hard drive to store sky patrol data. With 8.3 megabyte images this limits the number 
of images which will fit on a disk. For this reason and the increased sensitivity LOTIS sky patrol data currently have a $30 \mathrm{~s}$ integration time.

The sky patrol data can be used as template images to compare against GRB images. Since the full available sky is covered twice each night the data also covers GRB positions which have no real-time trigger but do have delayed localizations such as IPN data, BeppoSAX data, and XTE data. The sky patrol data will also be analyzed to search for other transient phenomena such as novae, supernovae, and solar system objects. It will also be used to identify and catalog unknown variable objects (rapid).

\section{Gamma-Ray Burst Triggers}

When the LOTIS sky patrol is interrupted due to a GCN GRB trigger the current process is stopped and the CCDs are flushed. The mount immediately slews to the location of the burst and begins imaging. If LOTIS observations of the GRB error box are not interrupted by subsequent or new triggers the telescope will continue to image the location for 20 minutes. When the 20 minute imaging is complete each camera obtains a set of 5 dark images. The sky patrol table is replaced by the a single entry which is the location of the GRB trigger. Therefore LOTIS continues to image the GRB error box for the remainder of the night.

If the initial burst trigger is interrupted by subsequent triggers with updated coordinates i.e. new trigger "types", the system checks the angular difference between the two triggers. If the difference is less than four degrees the system does not respond to the new trigger. If the difference is greater than four degrees the system will interrupt its current process and immediately slew to the new location while flushing the CCDs.

In the rare case where the initial trigger is interrupted by a trigger from a new burst the system will only respond if 10 minutes have passed from receipt of the first trigger. If 10 minutes have passed the system will immediately respond to the new trigger and keep both burst positions in the sky patrol for later observation. 


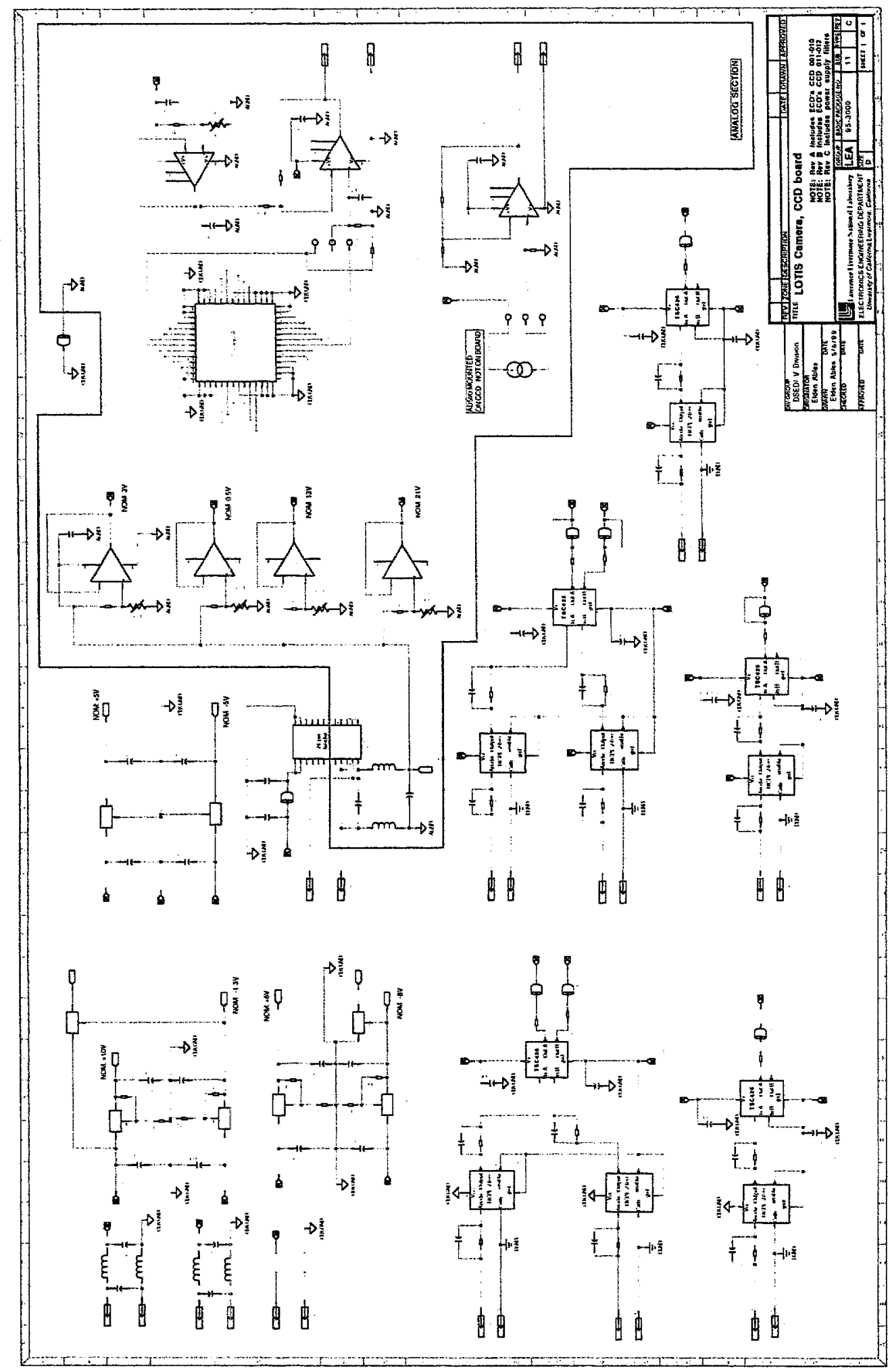

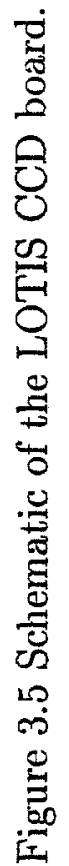




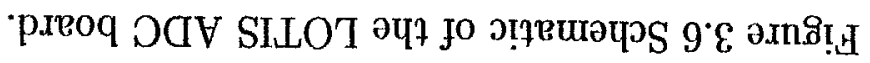

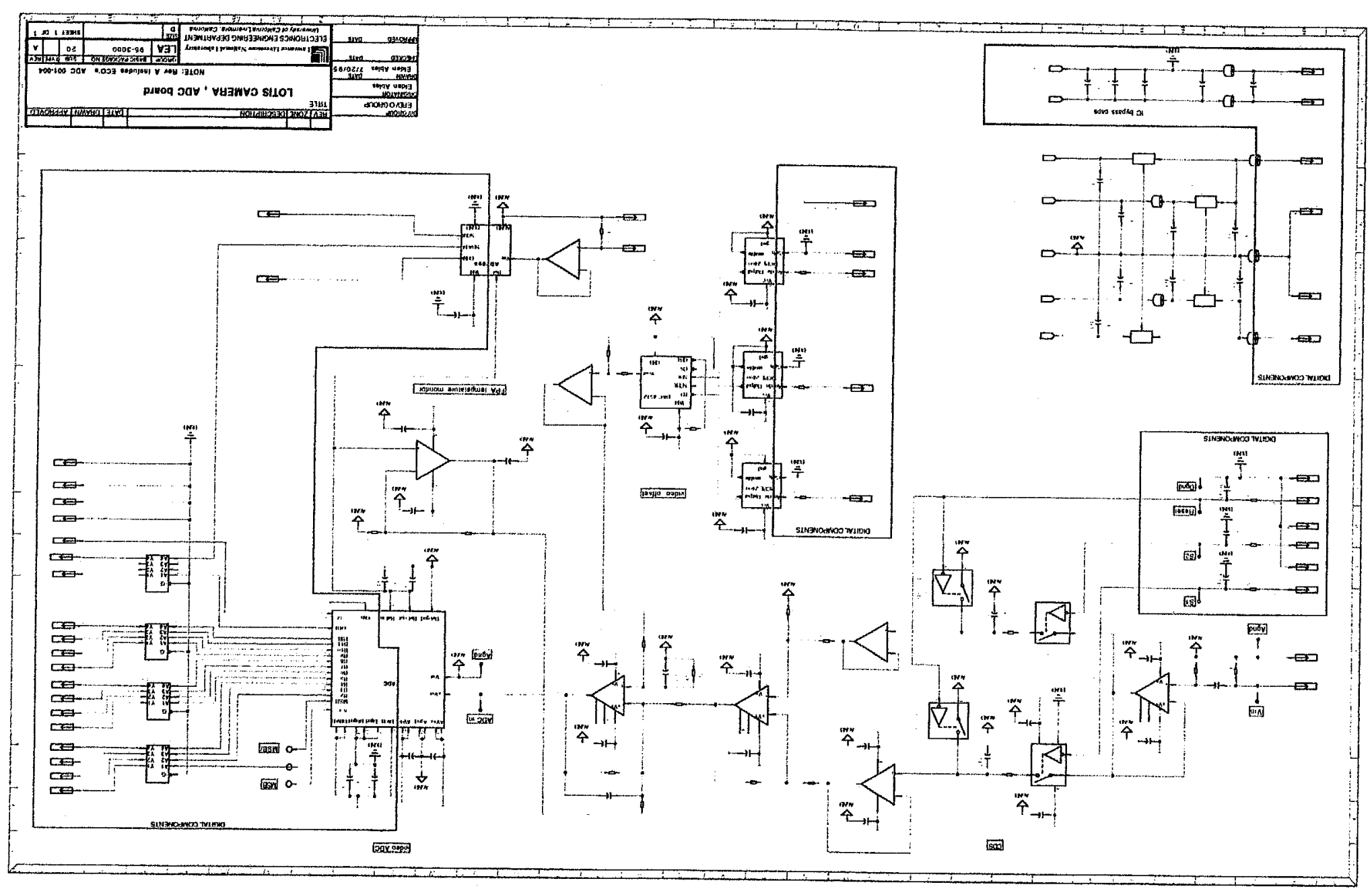




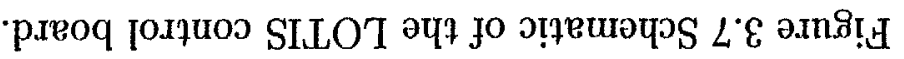

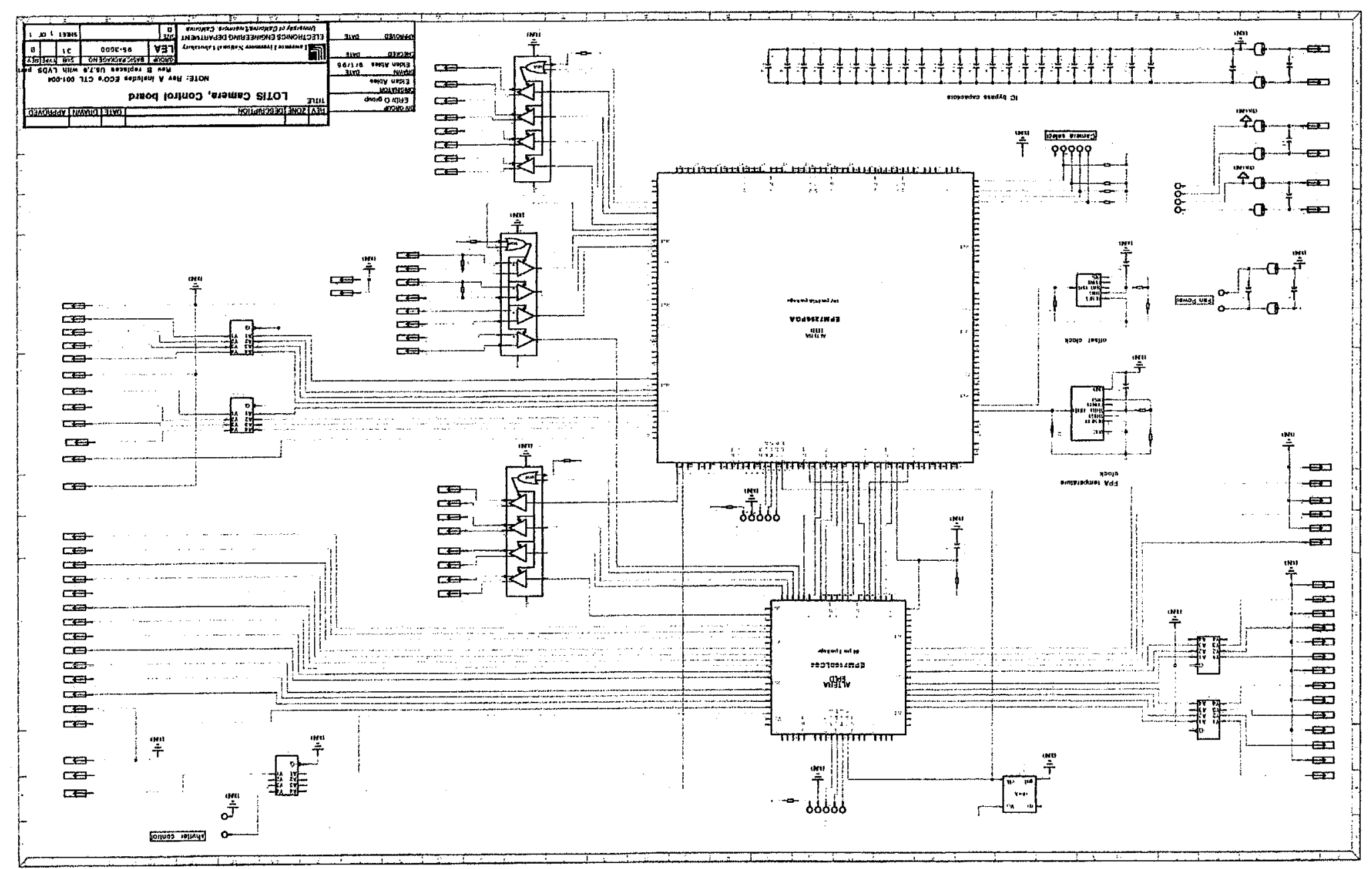




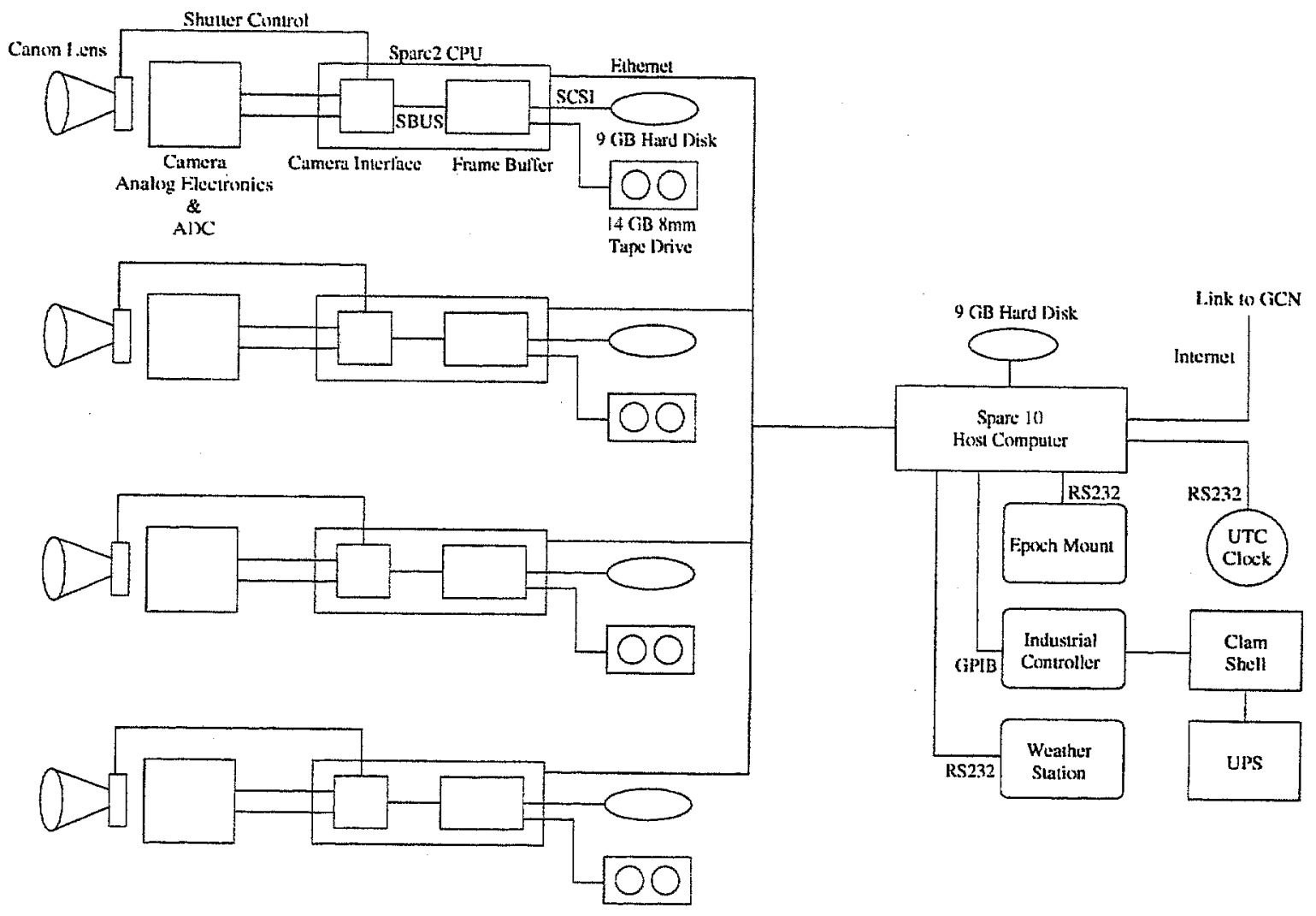

Figure 3.8 LOTIS data acquisition system. 


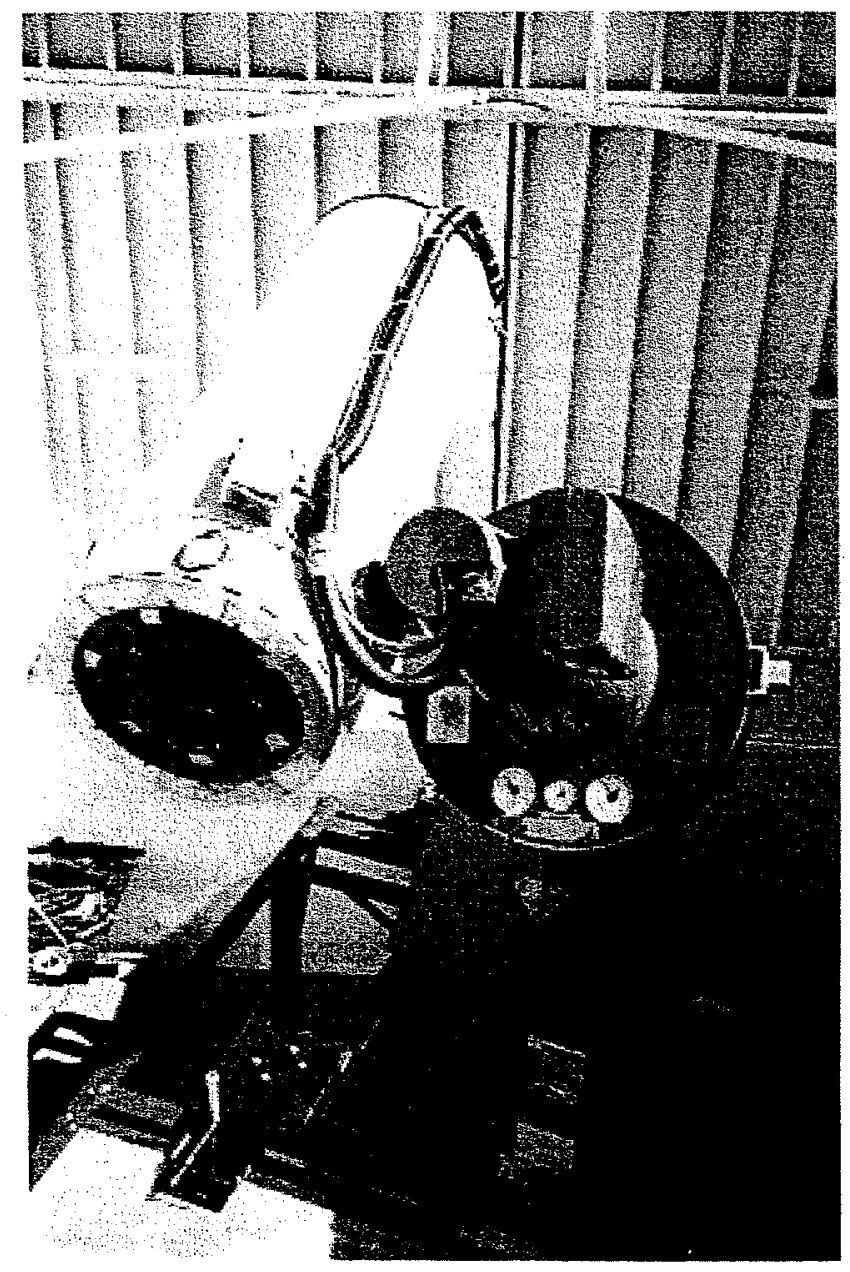

Figure 3.9 The Super-LOTIS telescope. 


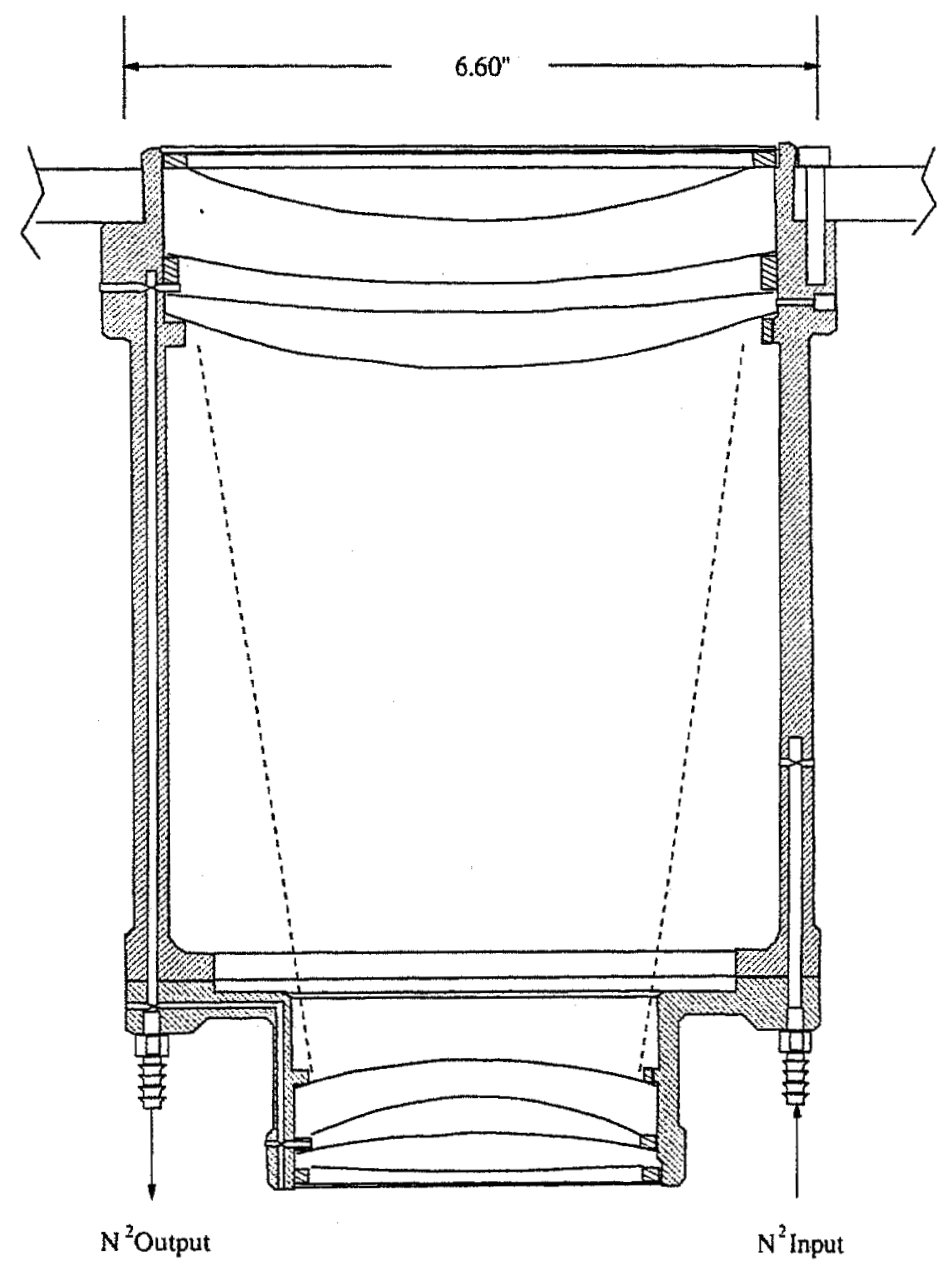

Figure 3.10 Design of the Super-LOTIS coma corrector. 


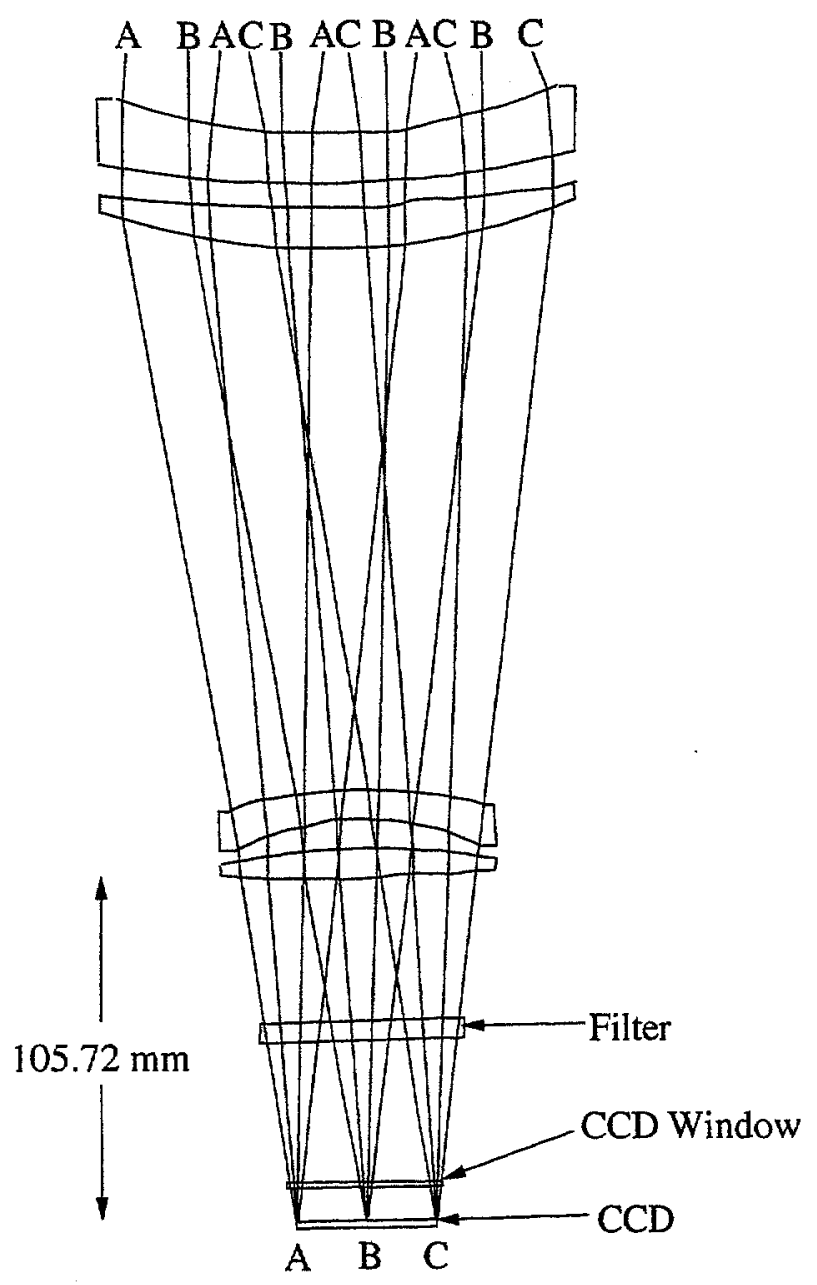

Figure 3.11 Super-LOTIS coma corrector optical light path. 


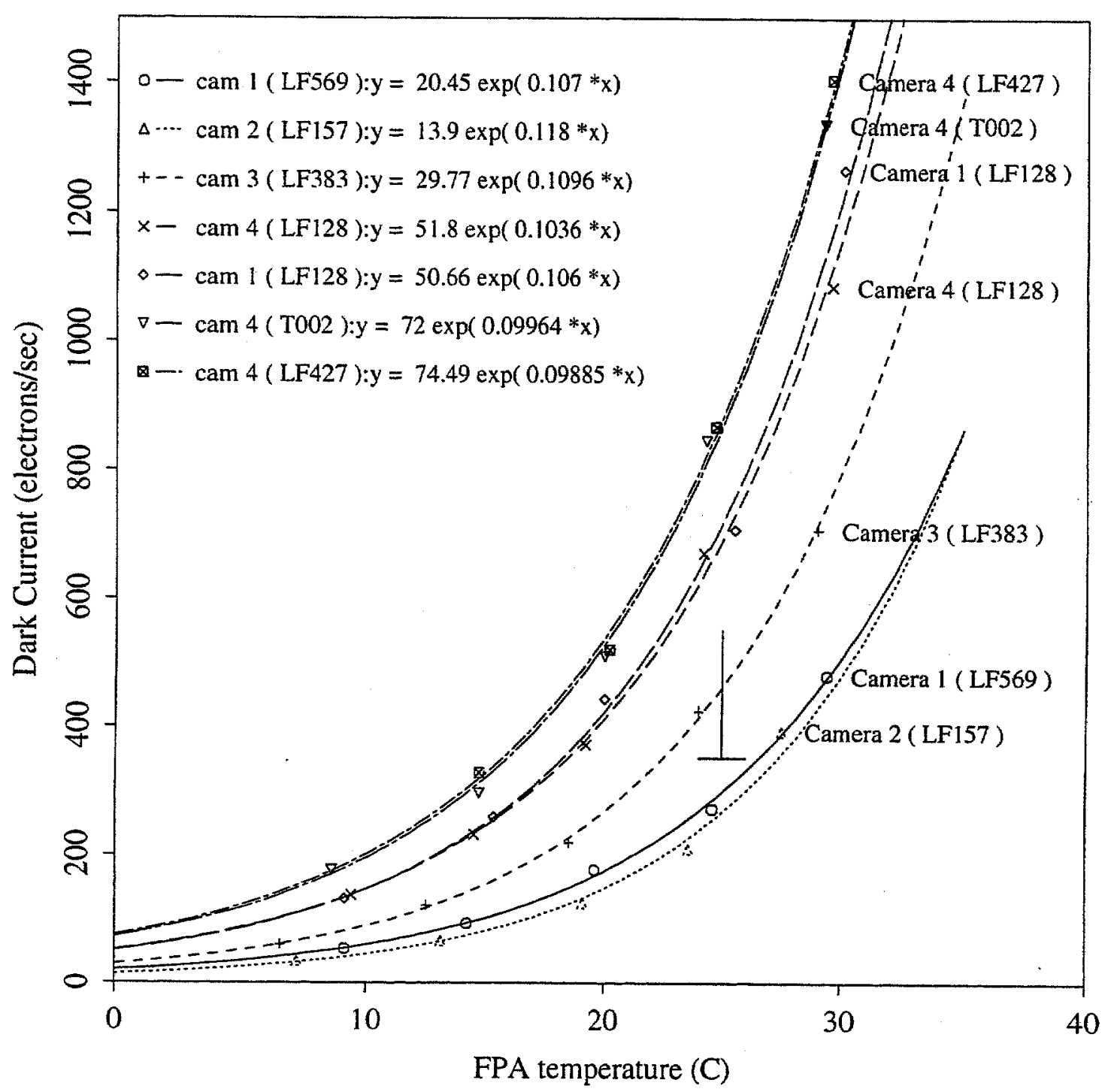

Figure 3.12 Loral 442A CCD dark current calibration data. 


\section{CHAPTER 4}

\section{RESULTS}

\section{GRB Triggers}

During more than 1330 possible nights of observation (October 1996 through May 2000), LOTIS has responded to 145 GCN triggers. Of these, 32 were updated coordinates for previous events. Particle events within the BATSE detectors and other false events accounted for 22 triggers. Soft gamma-ray repeaters (SGRs) added 16 triggers. The remaining 75 triggers were unique GRB events; a rate of approximately one unique GRB event every 17.7 days.

Each of the SGR triggers was analyzed in a manner similar to the GRB events. However, because of the recurrent nature of SGRs, they have been precisely localized by many IPN observations. The search for optical counterparts of the SGRs was restricted to a small area. LOTIS detected no optical counterparts for these SGR events. SGRs will not be discussed further.

The quality of the LOTIS temporal and spatial "coverage" of a given event depends on five factors: observing conditions, LOTIS response time, difference between the initial and final coordinates, size of the final error box, and the duration of the GRB. Figures 4.1, 4.2, 4.3, and 4.4 show histograms of LOTIS unique events versus the last four of these factors. In Figure 4.1 the LOTIS response time has been clipped to $20 \mathrm{~s}$ which shows only the events for which an Original GCN trigger was received. SGRs account for 16 events in the first bin of GRB durations (Figure 4.3).

Table II. lists the 13 events for which LOTIS achieved the best overall coverage. Each of these events is discussed in detail in the following sections. The last column lists the approximate limiting magnitude for each event.

\section{Data Presentation}

The BATSE light curves presented here use the $64 \mathrm{~ms}$ LAD data available in the public data archive. The four energy channels have been summed. The rates are 


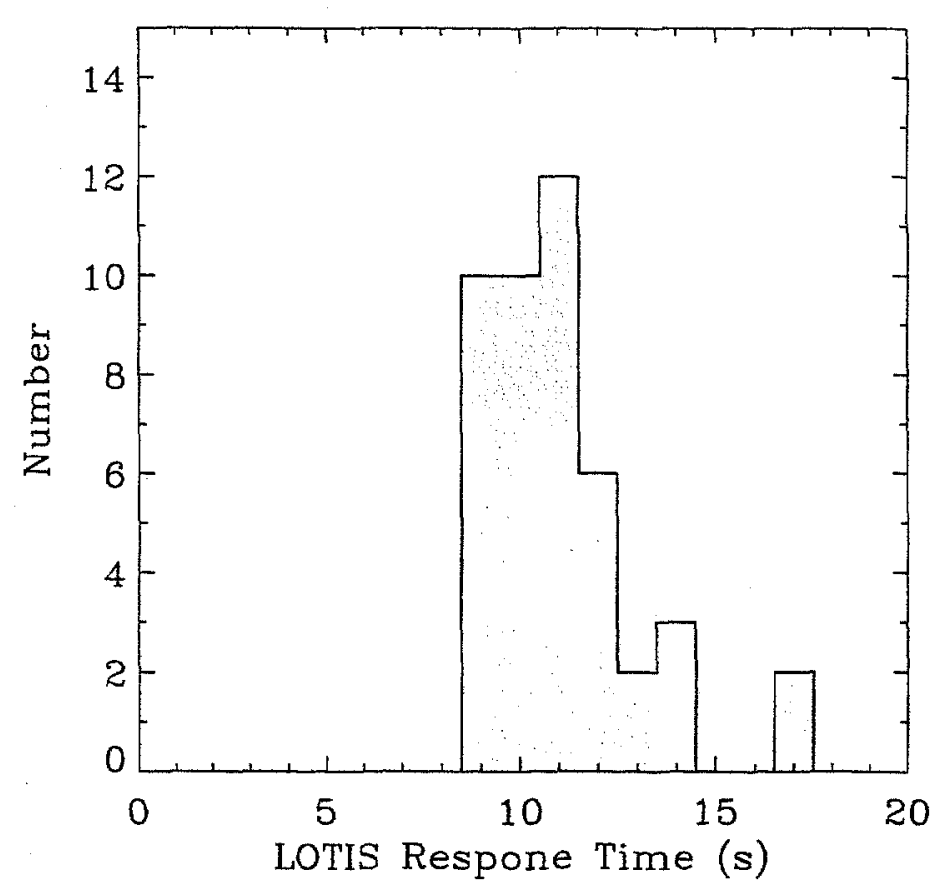

Figure 4.1 Histogram of LOTIS response times.

dependent on the number of detectors summed and therefore should not be interpreted as a scale for absolute flux. Peak photon flux values for each burst are given in Table Ir.

Throughout the text and in the coverage plots the standard notation for equatorial coordinates is used, i.e. $\alpha$ for right ascension and $\delta$ for declination. Unless otherwise noted all coordinates are equinox J2000.0. The BATSE $1 \sigma$ (68\% confdence) and $3 \sigma$ (99.7\% confidence) error circles are shown on each plot. The radii for these error circles were calculated using the method described below.

Figure 4.5 shows the BATSE burst location error model used in this analysis (Briggs et al. 1999b). This azimuthally symmetric error distribution is an improvement to the "minimal" model introduced by Meegan et al. (1996). In the minimal model the total error $\sigma_{t o t}$ is calculated by adding a constant 1:6 systematic error $\sigma_{\text {sys }}$ to the statistical error $\sigma_{\text {stat }}$ in quadrature,

$$
\sigma_{\text {tot }}=\left(\sigma_{\text {stat }}^{2}+1.6^{2}\right)^{1 / 2}
$$


The error model of Briggs et al. (1999b) is a two component model which consists of a "core" and a "tail". The core contains $78 \pm 0.08 \%$ of the integrated probability. Both components are based on the Fisher probability density function (Fisher et al. 1987) given by,

$$
p_{F}(\gamma) d \Omega=\frac{\kappa}{2 \pi\left(\mathrm{e}^{\kappa}-\mathrm{e}^{-\kappa}\right)} \mathrm{e}^{\kappa \cos \gamma} d \Omega
$$

where $\gamma$ is the angle between the measured location and the true location and $\kappa$ is the concentration parameter defined to make $\sigma_{\text {tot }}$ contain $68 \%$ of the probability. For $\sigma_{\text {tot }}$ in radians,

$$
\kappa=\frac{1}{\left(0.66 \sigma_{t o t}\right)^{2}}
$$

The widths of the core $(i=1)$ and tail $(i=2)$ are given by,

$$
\sigma_{t o t, i}=\left(\sigma_{s t a t}^{2}+\sigma_{s y s, i}^{2}\right)^{1 / 2}
$$

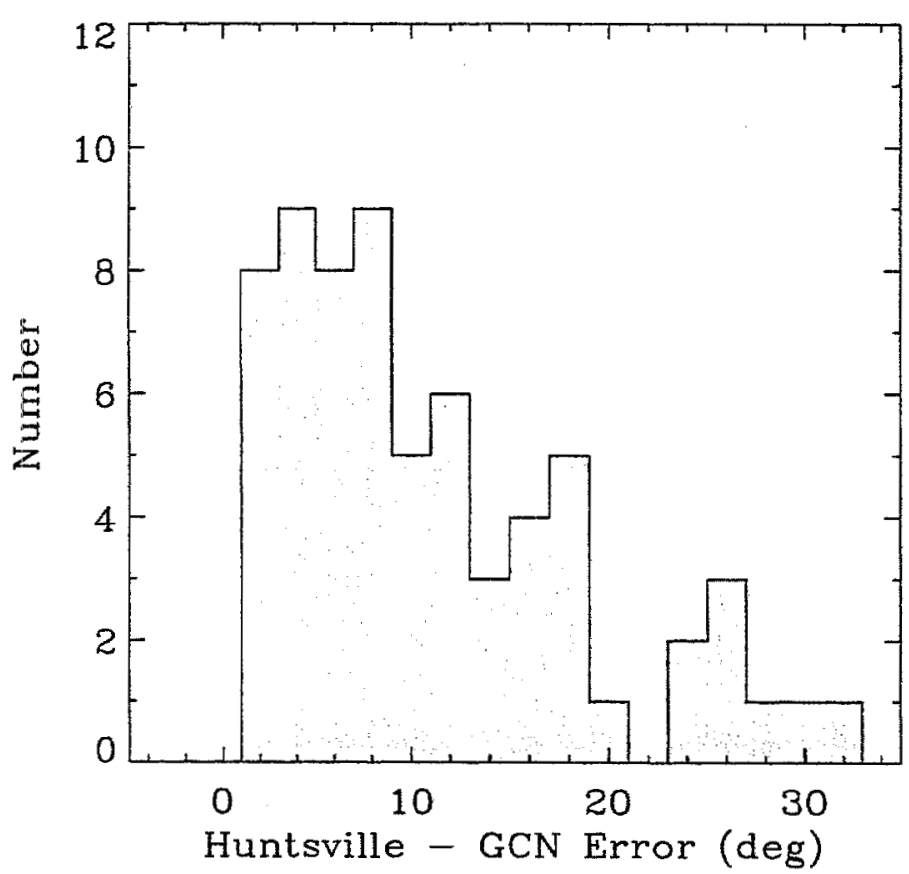

Figure 4.2 Histogram of errors between the Original GCN coordinates and the final Huntsville coordinates. 


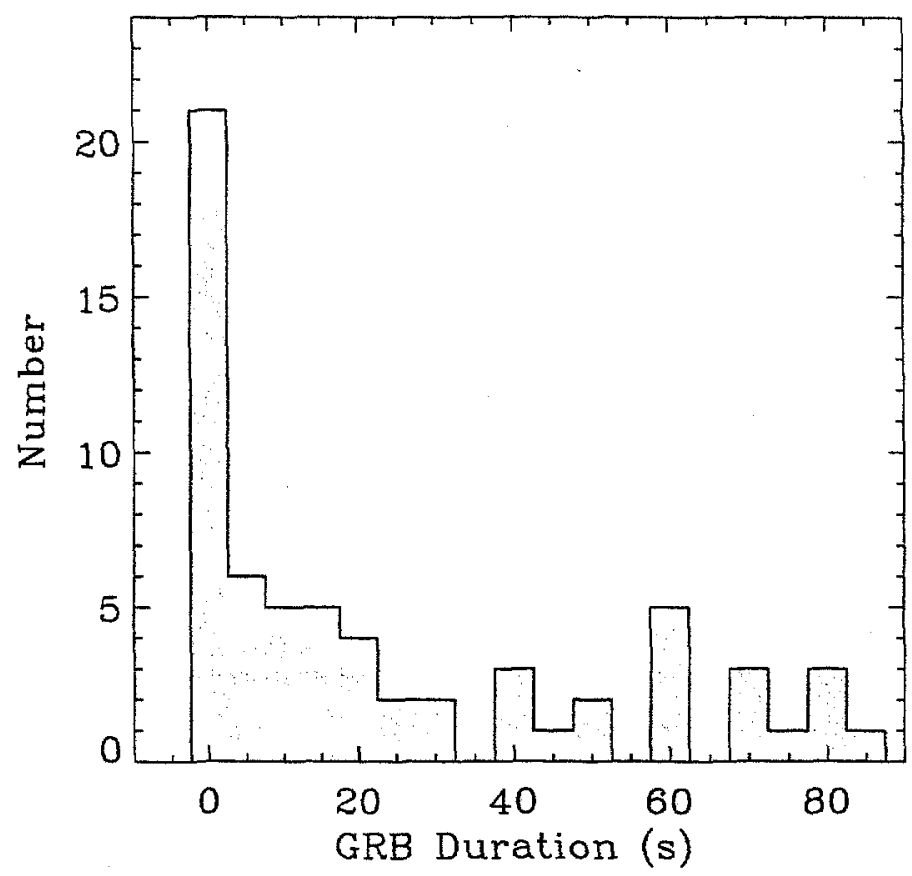

Figure 4.3 Histogram of GRB durations for LOTIS events. The first bin is contaminated by 16 SGR events.

where $\sigma_{s y s, 1}=1.85 \pm 0^{\circ} 16$ and $\sigma_{s y s, 2}=5^{\circ} 1_{-0.6}^{+0.8}$. The $1 \sigma$ and $3 \sigma$ error radii are defined as the central $68 \%$ and $99.7 \%$ of the sum of the core and tail.

For each of the bursts discussed below we include the "probability content" of the LOTIS coverage. We calculate the probability content by integrating the two component error model described above over the two dimensional area covered by the LOTIS cameras. The probability content is essentially the probability that LOTIS imaged the burst location. Only the final Huntsville error circle is used in calculating this probability. The IPN data and other localizations are not included in the probability.

\section{GCN Trigger Types}

The success of the LOTIS experiment is dependent on the availability of realtime GRB triggers. The single source for these triggers is the GRB Coordinates Network (GCN; Barthelmy et al. 2000). Prior to the de-orbit of CGRO, GCN inter- 
cepted real-time telemetry from the spacecraft and used the BATSE data to calculate approximate burst positions. The real-time data is from the $1.024 \mathrm{~s}$ sampling of the four energy channels of the LADs. In recent years several additional spacecraft have added GCN to their pipeline for dissemination of GRB coordinates, although none in real-time.

Table III. shows the 13 types of GCN triggers available or planned prior to the de-orbit of CGRO. The GCN software continuously scans the incoming BATSE data for a high trigger flag. In this mode the system is also calculating the background rates in a sliding $10 \mathrm{~s}$ window. When the trigger flag is high the program determines which sample contains a rate increase of $5 \sigma$ above the background. This threshold is in place to allow adequate signal-to-noise in calculating the location. If no sample meets this threshold requirement the system waits for $10 \mathrm{~s}$ or until the threshold is met before calculating the coordinates.

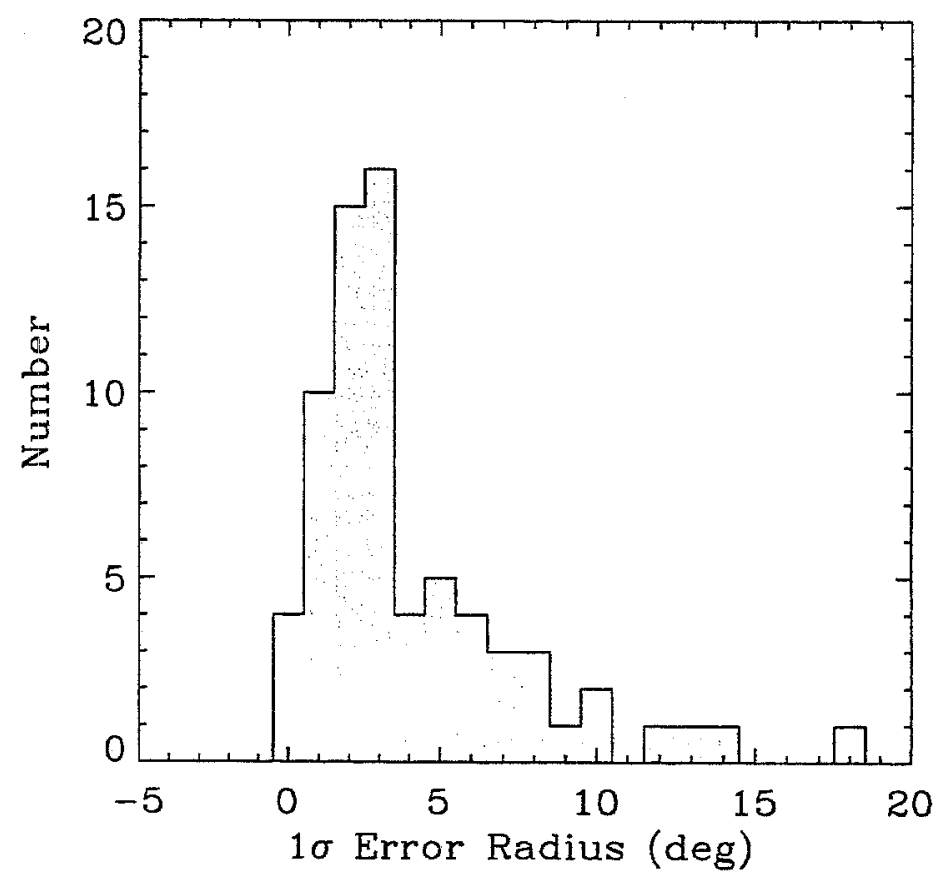

Figure 4.4 Histogram of $1 \sigma$ error radii for LOTIS events. 
Table II. LOTIS GRB events with good coverage.

\begin{tabular}{ccccrcr}
\hline Date & $\begin{array}{c}\text { BATSE } \\
\text { Trig. }\end{array}$ & $\begin{array}{c}F_{p}(1024 \mathrm{~ms}) \\
\left(\gamma \mathrm{cm}^{-2} \mathrm{~s}^{-1}\right)\end{array}$ & $\begin{array}{c}S / 10^{-7} \\
\left(\mathrm{erg} \mathrm{cm}^{-2}\right)\end{array}$ & $\begin{array}{c}\mathrm{T}_{90} \\
(\mathrm{~s})\end{array}$ & $\begin{array}{c}\mathrm{T}_{\text {LOTIS }} \\
(\mathrm{s})\end{array}$ & $\begin{array}{r}\text { LOTIS } \\
\text { Limit }\end{array}$ \\
\hline \hline 961017 & 5634 & 1.98 & 5.07 & 1.2 & 11.0 & 11.5 \\
961220 & 5719 & 1.60 & 18.11 & 9.8 & 9.0 & 11.5 \\
970223 & 6100 & 16.84 & 968. & 16.3 & 11.5 & 11.0 \\
970714 & 6307 & 1.32 & 17.09 & 2.0 & 14.06 & 11.3 \\
970919 & 6388 & 0.77 & 22.49 & 20.9 & 11.8 & 11.5 \\
971006 & 6414 & 1.79 & 258. & 48.1 & 17.1 & 12.1 \\
971227 & 6546 & 2.11 & 9.25 & 6.8 & 10.0 & 12.3 \\
990129 & 7360 & 4.99 & 585. & 200.0 & 140.8 & 14.5 \\
990308 & 7457 & 1.26 & 164. & 50.0 & 132.1 & 13.5 \\
990316 & 7475 & 3.67 & 529. & 40.0 & 13.6 & 14.3 \\
990413 & 7518 & 2.57 & 68.13 & 15.0 & 13.0 & 14.0 \\
990803 & 7695 & 12.19 & 1230. & 28.0 & 15.0 & 14.5 \\
990918 & 7770 & 3.17 & 25.21 & 6.5 & 8.3 & 14.3 \\
\hline
\end{tabular}

The Original GCN coordinates are calculated using the first one or two $1.024 \mathrm{~s}$ rate samples and an ideal response function, i.e. there is no correction for photon scattering off the spacecraft or the atmosphere. Because of the ideal response assumption and the limited data the Original GCN coordinates generally have the largest error.

The Final GCN coordinates are calculated using up to $32 \mathrm{~s}$ of data. The calculation uses only data which exceed the minimum threshold of $5 \sigma$ above the background. Since the counting statistics are improved (for bursts which last longer than $2 \mathrm{~s}$ ) the location error for the Final GCN coordinates is typically smaller than the error for the Original GCN coordinates. However an ideal response is still assumed and the systematic errors often dominate. 


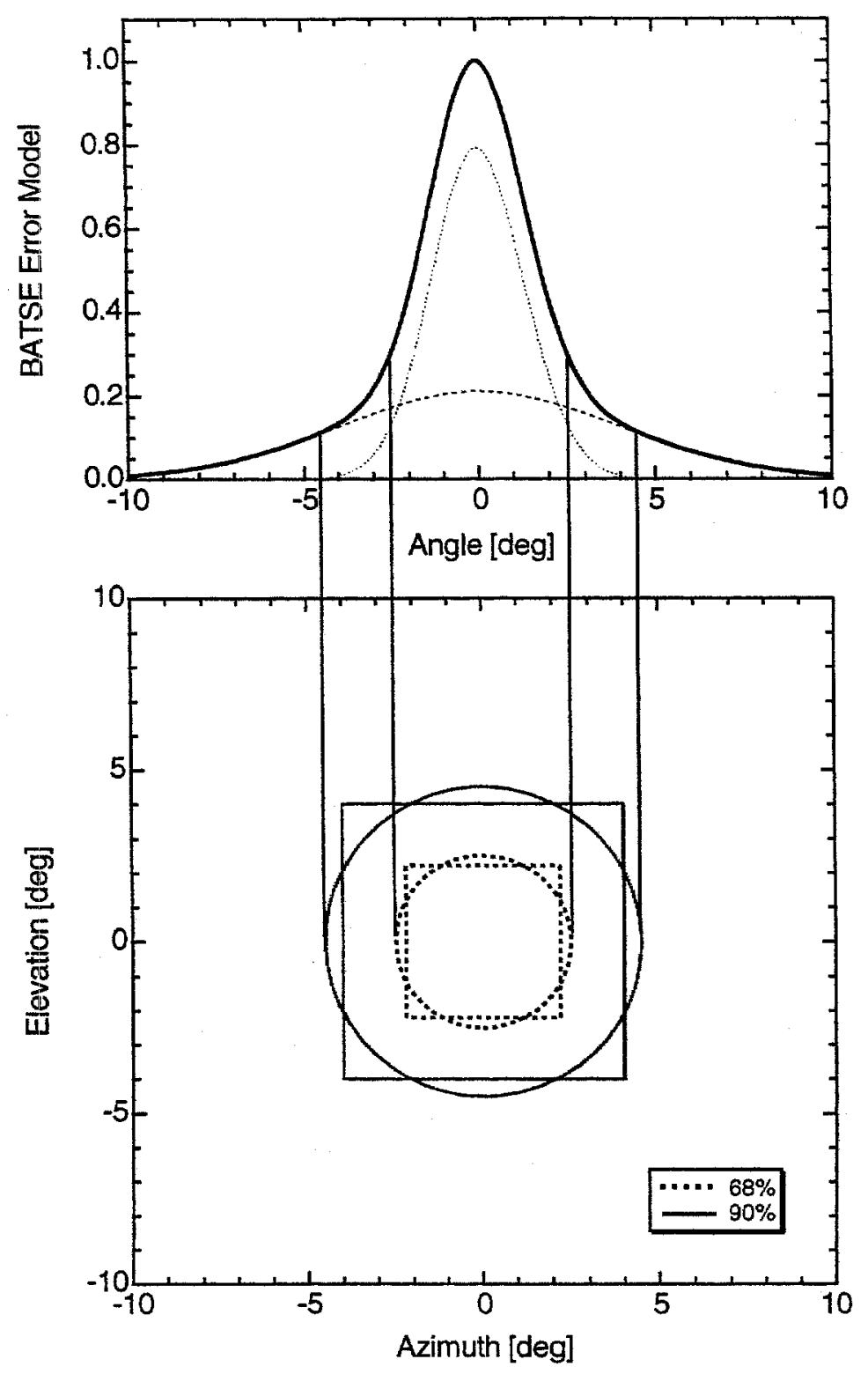

Figure 4.5 The BATSE burst location error model. Two Fisher distributions; a core containing $78 \%$ of the integrated probability and a tail (Courtesy R. M. Kippen).

The LOCFAST GCN trigger is based on the LOCBURST algorithm for calculating burst coordinates (see below). However this trigger type was not implemented before CGRO was de-orbited.

The Light Curve GCN trigger contains light curve data only in several formats and energy ranges. This trigger is only sent to the GCN web page. 
Table III. GCN Trigger Types.

\begin{tabular}{llll} 
Source & Time Delay & Error Box Size & Rate \\
\hline Original & $5 \mathrm{~s}$ & $6^{\circ}-20^{\circ}$ & $1 /$ day \\
Final & $37 \mathrm{~s}$ & $6^{\circ}-18^{\circ}$ & $1 /$ day \\
LOCFAST & $2 \mathrm{~min}$ & $3^{\circ}-5^{\circ}$ & $50 /$ year \\
Light Curve & $5 \mathrm{~min}$ & $\mathrm{n} / \mathrm{a}$ & $1 /$ day \\
MAXBC & $10 \mathrm{~min}$ & $5^{\circ}-20^{\circ}$ & $1 /$ day \\
LOCBURST & $15-30 \mathrm{~min}$ & $4^{\circ}-8^{\circ}$ & $10 /$ month \\
COMPTEL & $15-30 \mathrm{~min}$ & $3^{\circ}-5^{\circ}$ & $5 /$ year \\
RXTE-ASM & $1-2 \mathrm{hr}$ & $4^{\prime} \times 15^{\prime}-150^{\prime}$ & $8 /$ year \\
SAX-WFC & $2-3 \mathrm{hr}$ & $6^{\prime}-20^{\prime}$ & $8 /$ year \\
RXTE-PCA & $2-5 \mathrm{hr}$ & $6^{\prime}-40^{\prime}$ & $6 /$ year \\
SAX-NFI & $12-48 \mathrm{hr}$ & $100^{\prime \prime}$ & $4 /$ year \\
IPN & $0.5-3 \mathrm{day}$ & $4^{\prime} \times 4^{\circ}-8^{\circ}$ & $2 /$ month \\
ALEXIS & $12 \mathrm{hr}$ & 0.6 & $20 /$ year \\
\hline
\end{tabular}

The MAXBC (MAXimum Burst Channel) GCN coordinates are calculated using the 16 highest peak count rates in the 10 min interval following the on-board trigger. This trigger type was implemented to account for bursts which occurred during telemetry gaps when real-time data was unavailable.

The LOCBURST GCN coordinates require human intervention and therefore the delay between the burst and coordinate dissemination is longer but the location error is considerably less. The GCN Original trigger notifies the on-call personnel at NASA/Marshall Space Flight Center (MSFC). The location algorithm takes into account the true response of the BATSE detectors including scattering and allows interactive determination of the background. 
The COMPTEL GCN coordinates are calculated if the burst occurred in the field-of-view of the COMPTEL instrument (on-board CGRO) and there is a $4 \sigma$ detection by that instrument.

GCN trigger types from additional spacecraft require some human intervention by the instrument team. The RXTE-ASM, RXTE-PCA, BeppoSAX-WFC, and BeppoSAX-NFI GCN coordinates are calculated using data from the Rossi X-ray Timing Explorer All Sky Monitor and Proportional Counter Array and the BeppoSAX Wide Field Cameras and Narrow Field Instruments. The ALEXIS GCN coordinates provide data on UV transients which may not be associated with a GRB.

The IPN GCN coordinates are calculated using time-of-arrival data from two or more widely separated spacecraft. The IPN localizations are now automated and the delay is limited by the telemetry time from the various spacecraft. Data from two späcecraft produce a narrow error annulus while data from three produce crossing annuli which result in a precise error box. The coordinates distributed automatically are preliminary and subject to change (usually becoming more narrow) after human intervention. The data presented in the sections below uses the final IPN data.

Finally, the BATSE team releases the final Huntsville coordinates for a GRB a few days after the burst. These coordinates use the most rigorous algorithm for calculating burst positions. The final Huntsville coordinates are incorporated into the BATSE catalog of GRBs.

\section{GRB 961017}

GRB 961017 (Trig. 5634) was detected by BATSE on October 17, 1996 at 11:16:43.59 UTC (03:16:43.59 PST). LOTIS received the Original GCN coordinates of $(\alpha, \delta ; \mathrm{J} 2000.0)=(39: 87,-10.25)$ at 11:16:49.0 UTC, approximately 5.4 seconds after the trigger. After slewing to the coordinates $(\sim 6.6 \mathrm{~s})$ LOTIS began imaging the Original GRB position at 11:16:54.6 UTC, just 11.0 seconds after the BATSE trigger.

Figure 4.6 illustrates the LOTIS response time to GRB 961017. The solid line represents the BATSE gamma-ray ( $>20 \mathrm{keV}$ ) light curve with a duration of $\sim 3 \mathrm{~s}$. 
The grey shaded area represents the $10 \mathrm{~s}$ integration time of the first LOTIS image. LOTIS began imaging approximately 8.0 seconds after gamma-ray emission ceased and continued to image the area for the next 20 minutes.

Figure 4.7 illustrates the LOTIS coverage of the final Huntsville error circle centered at $(\alpha, \delta ; \mathrm{J} 2000.0)=\left(37.16,-10^{\circ} .68\right)$, approximately 2.7 from the Original GCN coordinates. The inner and outer ellipses represent the BATSE $1 \sigma$ (2.89 statistical error) and $3 \sigma$ error circles. The narrow arc represents the IPN annulus calculated using data from BATSE and the Ulysses GRB Monitor. The annulus is centered at $(\alpha, \delta ; \mathrm{J} 200.0)=\left(354^{\circ} .974,-32^{\circ} .029\right)$ and has a radius and width of $40^{\circ} .754$ and $\pm 0^{\circ} .026$ respectively. The pseudo-rectangles represent the area covered by the LOTIS cameras. The center of the LOTIS camera array does not align with the center of the BATSE error circles because LOTIS responded to the Original GCN trigger and the error circles are centered on the final Huntsville coordinates. The probability content of the LOTIS coverage of GRB 961017 is $97.86 \%$. The IPN annulus is not included in this probability.

\section{GRB 961220}

GRB 961220 (Trig. 5719) was detected by BATSE on December 20, 1996 at 05:21:51.05 UTC (Dec. 19, 21:21:51.05 PST). LOTIS received the Original GCN coordinates of $(\alpha, \delta ; \mathrm{J} 2000.0)=(10: 7,+17.52)$ at 05:21:56.0 UTC, approximately 5.0 seconds after the trigger. After slewing to the coordinates $(\sim 4.0 \mathrm{~s})$ LOTIS began imaging the Original GRB position at 05:22:00.0 UTC, just 9.0 seconds after the BATSE trigger.

Figure 4.8 illustrates the LOTIS response time to GRB 961220. The solid line represents the BATSE gamma-ray light curve $(>20 \mathrm{keV})$ with a duration of $\sim 15 \mathrm{~s}$. The grey shaded area represents the $10.0 \mathrm{~s}$ integration time of the first LOTIS image. LOTIS began imaging during the prompt gamma-ray emission and continued to image the area for the next 20 minutes.

Figure 4.9 illustrates the LOTIS coverage of the final Huntsville error circle centered at $(\alpha, \delta ; \mathrm{J} 2000.0)=(6 \circ 9,+17.33)$, approximately 3.6 from the Original GCN 
coordinates. The inner and outer ellipses represent the BATSE $1 \sigma$ (1.47 statistical error) and $3 \sigma$ error circles. The pseudo-rectangles represent the area covered by the LOTIS cameras. The center of the LOTIS camera array does not align with the center of the BATSE error circles because LOTIS responded to the Original GCN trigger and the error circles are centered on the final Huntsville coordinates. The probability content of the LOTIS coverage of GRB 961220 is $97.94 \%$.

\section{GRB 970223}

GRB 970223 (Trig. 6100) was detected by BATSE on February 23, 1997 at 08:26:17.67 UTC (00:26:17.67 PST). LOTIS received the Original GCN coordinates of $(\alpha, \delta ; \mathrm{J} 2000.0)=\left(144.85,+36^{\circ} 13\right)$ at 08:26:23.5 UTC, approximately 5.8 seconds after the trigger. After slewing to the coordinates ( $\sim 5.7 \mathrm{~s})$ LOTIS began imaging the Original GRB position at 08:26:29.2 UTC, just 11.5 seconds after the BATSE trigger.

Figure 4.10 illustrates the LOTIS response time to GRB 970223. The solid line represents the BATSE batse gamma-ray light curve ( $>20 \mathrm{keV})$ with a duration of $\sim 30 \mathrm{~s}$. The grey shaded area represents the $10.0 \mathrm{~s}$ integration time of the first LOTIS image. 'LOTIS began imaging approximately 11.5 seconds after gamma-ray emission began resulting in an optical image simultaneous with the gamma-ray burst. LOTIS continued to image the area for the next 20 minutes.

Figure 4.11 illustrates the LOTIS coverage of the final Huntsville error circle centered at $(\alpha, \delta ; \mathrm{J} 2000.0)=\left(142^{\circ} 48,+35^{\circ} 43\right)$, approximately 2.0 from the Original GCN coordinates. The inner and outer ellipses represent the BATSE $1 \sigma$ (0:73 statistical error) and $3 \sigma$ error circles. The narrow arc represents the IPN annulus calculated using data from BATSE and the Ulysses GRB Monitor. The annulus is centered at $(\alpha, \delta ; \mathrm{J} 2000.0)=\left(167.407,+36^{\circ} 399\right)$ and has a radius and width of $18^{\circ} .813$ and $\pm 0: 029$ respectively. The pseudo-rectangles represent the area covered by the LOTIS cameras. The probability content of the LOTIS coverage of GRB 970223 is $99.09 \%$. This probability does not include the IPN data. A full description of the LOTIS results from GRB 970223 is presented in Park et al. (1997c). 


\section{GRB 970714}

GRB 970714 (Trig. 6307) was detected by BATSE on July 14, 1997 at 10:46:57.57 UTC (03:46:57.57 PST). LOTIS received the Original GCN coordinates of $(\alpha, \delta ; \mathrm{J} 2000.0)$ $=(1: 54,-23.22)$ at 10:47:04.0 UTC, approximately 6.4 seconds after the trigger. After slewing to the coordinates $(\sim 7.6 \mathrm{~s})$ LOTIS began imaging the Original GRB position at 10:47:11.6 UTC, just 14.0 seconds after the BATSE trigger.

Figure 4.12 illustrates the LOTIS response time to GRB 970714. The solid line represents the BATSE gamma-ray light curve $(>20 \mathrm{keV})$ with a duration of $\sim 1 \mathrm{~s}$. The grey shaded area represents the $10.0 \mathrm{~s}$ integration time of the first LOTIS image. LOTIS began imaging approximately 13.0 seconds after gamma-ray emission ceased and continued to image the area for the next 20 minutes.

Figure 4.13 illustrates the LOTIS coverage of the final Huntsville error circle centered at $(\alpha, \delta ; \mathrm{J} 2000.0)=\left(5.87,-29^{\circ} 14\right)$, approximately $7^{\circ} .1$ from the Original GCN coordinates. The inner and outer ellipses represent the BATSE $1 \sigma$ (2.79 statistical error) and $3 \sigma$ error circles. The pseudo-rectangles represent the area covered by the LOTIS cameras. The probability content of the LOTIS coverage of GRB 970714 is $82.95 \%$.

GRB 970919

GRB 970919 (Trig. 6388) was detected by BATSE on September 19, 1997 at 03:45:02.17 UTC (Sep. 18, 20:45:02.17 PST). LOTIS received the Original GCN coordinates of $(\alpha, \delta ; \mathrm{J} 2000.0)=\left(196^{\circ} .12,+50^{\circ} .48\right)$ at 03:45:07.5 UTC, approximately 5.3 seconds after the trigger. After slewing to the coordinates ( $\sim 6.6 \mathrm{~s})$ LOTIS began imaging the Original GRB position at 03:45:14.0 UTC, just 11.8 seconds after the BATSE trigger.

Figure 4.14 illustrates the LOTIS response time to GRB 970919. The solid line represents the BATSE gamma-ray light curve $(>20 \mathrm{keV})$ with a duration of $\sim 20 \mathrm{~s}$. The grey shaded area represents the $10.0 \mathrm{~s}$ integration time of the first LOTIS image. LOTIS began imaging during prompt gamma-ray emission and continued to image the area for the remainder of the night. 
Figure 4.15 illustrates the LOTIS coverage of the final Huntsville error circle centered at $(\alpha, \delta ; J 2000.0)=\left(195^{\circ} 03,+45^{\circ} .47\right)$, approximately 5.1 from the Original GCN coordinates. The inner and outer ellipses represent the BATSE $1 \sigma\left(3.0^{\circ}\right.$ statistical error) and $3 \sigma$ error circles. The pseudo-rectangles represent the area covered by the LOTIS cameras. The probability content of the LOTIS coverage of GRB 970919 is $91.27 \%$.

\section{GRB 971006}

GRB 971006 (Trig. 6414) was detected by BATSE on October 6, 1997 at 05:16:57.44 UTC (Oct. 5, 21:16:57.44 PST). LOTIS received the Original GCN coordinates of $(\alpha, \delta ; \mathrm{J} 2000.0)=(241: 14,+49.23)$ at 05:17:06.6 UTC, approximately 9.2 seconds after the trigger. After slewing to the coordinates ( $7.9 \mathrm{~s}$ ) LOTIS began imaging the Original GRB position at 05:17:14.5 UTC, just 17.1 seconds after the BATSE trigger.

Figure 4.16 illustrates the LOTIS response time to GRB 971006. The solid line represents the BATSE gamma-ray light curve $(>20 \mathrm{keV})$ with a duration of $\sim 150 \mathrm{~s}$. The grey shaded area represents the $10.0 \mathrm{~s}$ integration time of the first LOTIS image. LOTIS began imaging during prompt gamma-ray emission and continued to image the area for the remainder of the night.

Figure 4.17 illustrates the LOTIS coverage of the final Huntsville error circle centered at $(\alpha, \delta ; \mathrm{J} 2000.0)=\left(249^{\circ} 79,+53^{\circ} .29\right)$, approximately 6.8 from the Original GCN coordinates. The inner and outer ellipses represent the BATSE $1 \sigma$ (0.6 statistical error) and $3 \sigma$ error circles. The two narrow arcs represent the IPN annuli calculated using data from the BATSE, Ulysses and NEAR gamma-ray burst detectors. The BATSE/Ulysses annulus is centered at $(\alpha, \delta ; \mathrm{J} 2000.0)=\left(166^{\circ} 838,+15^{\circ} .53\right)$ and has a radius and width of 74.162 and $\pm 0: 051$ respectively. The BATSE/NEAR annulus is centered at $(\alpha, \delta ; \mathrm{J} 2000.0)=\left(252.371,-21^{\circ} .448\right)$ and has a radius and width of $75^{\circ} .317$ and \pm 0.372 respectively. The intersection of these annuli provides a precise localization of GRB 971006. The pseudo-rectangles represent the area covered by the LOTIS cameras. The probability content of the LOTIS coverage for this event 
is $50.41 \%$. This probability does not include the IPN data which would result in a probability content of $100 \%$. A description of the LOTIS results from GRB 971006 is presented in Williams et al. (1998)

\section{GRB 971227}

GRB 971227 (Trig. 6546) was detected by BATSE on December 27, 1997 at 08:23:06.72 UTC (00:23:06.72 PST). LOTIS received the Original GCN coordinates of $(\alpha, \delta ; \mathrm{J} 2000.0)=\left(197.53,+65^{\circ} .80\right)$ at 08:23:10.9 UTC, approximately 4.2 seconds after the trigger. After slewing to the coordinates ( $5.8 \mathrm{~s})$ LOTIS began imaging the Original GRB position at 08:23:16.7 UTC, just 10.0 seconds after the BATSE trigger (Park et al. 1997b).

Figure 4.18 illustrates the LOTIS response time to GRB 971227. The solid line represents the BATSE gamma-ray light curve $(>20 \mathrm{keV})$ with a duration of $\sim 7 \mathrm{~s}$. The grey shaded area represents the $10.0 \mathrm{~s}$ integration time of the first LOTIS image. LOTIS began imaging approximately 6.0 seconds after gamma-ray emission ceased and continued to image the area for the remaining six hours of darkness.

Figure 4.19 illustrates the LOTIS coverage of the final Huntsville error circle centered at $(\alpha, \delta ; \mathrm{J} 2000.0)=(190.00,+54.31)$, approximately $14^{\circ}$ from the Original GCN coordinates. The inner and outer ellipses represent the BATSE $1 \sigma$ (1:66 statistical error) and $3 \sigma$ error circles. The pseudo-rectangle represents the area covered by a single LOTIS camera (camera \#1). The upper left hand corner of the rectangle is the center of the $2 \times 2$ array of cameras directed toward the Original GCN coordinates. The area covered by the remaining three LOTIS cameras is not plotted for clarity. GRB 971227 was also detected by the SAX/WFC. The small circle represents the position $(\alpha, \delta ; \mathrm{J} 2000.0)=\left(194^{\circ} .371,+59^{\circ} .272\right)$ and $1 \sigma$ error circle $\left(10^{\prime}\right.$ radius $)$ determined by the SAX/WFC. The SAX coordinates of an afterglow detected by NFI at $(\alpha, \delta ; \mathrm{J} 2000.0)=\left(194^{\circ} .313,+59^{\circ} .401\right)(1.5$ radius error $) 14$ hours after the burst are within the SAX/WFC error circle. The probability content of the LOTIS coverage of GRB 971227 is 5.63\%. This probability does not include data IPN or BeppoSAX 
data which would increase the probability content to $100 \%$. A full description of the LOTIS results from GRB 971227 is presented in Williams et al. (1999a).

Figure 4.20 shows the LOTIS upper limits together with the gamma-ray and $\mathrm{x}$-ray data and the afterglow upper limits.

\section{GRB 980703}

GRB 980703 (Trig. 6891) was detected by BATSE on July 3, 1998 at 04:22:45.21 UTC (Jul. 2, 21:22:45.21 PST). LOTIS received the Original GCN coordinates of $(\alpha, \delta ; \mathrm{J} 2000.0)=(5.36,+14: 06)(\mathrm{J} 2000.0)$ at $04: 22: 51.40 \mathrm{UTC}$, approximately 6.19 seconds after the trigger. This trigger was received during twilight hours and the coordinates were below the horizon, therefore LOTIS did not respond to the trigger in real-time. LOTIS received the Final, MAXBC, and Locburst GCN coordinates of $(\alpha, \delta ; \mathrm{J} 2000.0)=\left(4.33,+16^{\circ} .49\right),(\alpha, \delta ; \mathrm{J} 2000.0)=\left(8.27,+32^{\circ} .44\right)$, and $(\alpha, \delta ; \mathrm{J} 2000.0)=\left(356^{\circ} .09,+13.65\right)$ at 04:24:36.76 UTC, 04:27:49.92 UTC, and 04:36:16.70 UTC respectively.

GRB 980703 was also detected by the RXTE/ASM which provided two intersecting long narrow error boxes. The intersection of these boxes localized the position to a $4^{\prime}$ radius error circle centered at $(\alpha, \delta ; \mathrm{J} 2000.0)=\left(23^{\mathrm{h}} 59^{\mathrm{m}} 05^{\mathrm{s}},+8^{\circ} 33^{\mathrm{m}} \cdot 6\right)$ (Levine et al. 1998). After further analysis this position was superseded by a more accurate localization consisting of a diamond shaped area with corners defined by $(\alpha, \delta ; \mathrm{J} 2000.0)$ : $\left(359^{\circ} 873,+8^{\circ} .653\right),\left(359^{\circ} .742,+8^{\circ} .573\right),\left(359^{\circ} 664,+8^{\circ} .459\right)$, and $\left(359^{\circ} .795,+8^{\circ} .540\right)$ (Smith et al. 1998).

Follow up observations by the SAX/NFI detected a previously unknown fading X-ray source within the RXTE/ASM error box (Galama et al. 1998a). The X-ray source (1SAX J2359.1+0835) was localized to a $50^{\prime}$ error circle centered at $(\alpha, \delta ; J 2000.0)=\left(23^{\mathrm{h}} 59^{\mathrm{m}} 07^{\mathrm{s}},+08^{\circ} 35^{\prime} 33^{\prime \prime}\right)$. Frail et al. (1998) reported the detection of possible radio and optical counterparts located at $(\alpha, \delta ; J 2000.0)=\left(23^{\mathrm{h}} 59^{\mathrm{m}} 6.67,+08^{\circ} 35^{\prime} 6^{\prime \prime} .7\right)$ coincident with the BeppoSAX X-ray source.

The first optical observations of the RXTE/ASM error box were obtained on July 4.06 (I band; Vreeswijk et al. 1998) and July 4.123 ( $\mathrm{R}$ band; Zapatero Osorio 
et al. 1998). Although the GRB position was not observable in real-time the LOTIS sky patrol covered the field on July 3.39, 5.03 hours after the burst (Park et al. 1998b). Therefore LOTIS was the first instrument to image the location. LOTIS also obtained sky patrol data from the previous and the following nights. The integration time used for these images was $30 \mathrm{~s}$. No flaring or fading sources were observed at $V \sim 15.0$ with a $5 \sigma$ detection threshold.

The optical afterglow light curve along with the LOTIS upper limit is shown in Figure 4.21. A full description of the LOTIS data from this burst is given in Park et al. (1999b).

\section{GRB 990129}

On the evening of January $28^{\text {th }}-29^{\text {th }}$ LOTIS responded to 4 GCN triggers: Trig. 7359 (MAXBC), Trig. 7360 (Original), Trig. 7360 (Final), and Trig. 7360 (Locburst). GRB 990129b (Trig. 7359) was detected by BATSE at 3:41:38.9 UTC (7:41:38.9 PST). This burst was a short single peaked burst lasting only 0.1 seconds. Due to insufficient counts an Original GCN trigger was not distributed. LOTIS received the MAXBC GCN coordinates of $(\alpha, \delta ; \mathrm{J} 2000.0)=\left(99^{\circ} 67,+16^{\circ} .08\right)$ at 3:52:20.2 UTC approximately 641 seconds after the BATSE trigger. LOTIS continued to image the MAXBC location for the next 180 minutes obtaining more than 150 images of this position. The final Huntsville coordinates of GRB $990129 \mathrm{~b}$ are $(\alpha, \delta ; \mathrm{J} 2000.0)=(100.97,-19.08)$ with a statistical error of 12.21 . Due to the large difference between the MAXBC coordinates and the Huntsville coordinates and the size of the $1 \sigma$ error box this is not considered a good LOTIS event.

GRB 990129 (Trig. 7360) was detected by BATSE at 5:15:50.9 UTC (9:15:50.9 PST). LOTIS received the Original GCN coordinates of $(\alpha, \delta ; J 2000.0)=(107.60,-1.73)$ at 5:15:56.7 UTC, approximately 5.8 seconds after the trigger. After slewing to the coordinates $(\sim 3.7 \mathrm{~s})$ LOTIS began imaging the Original GRB position at 5:16:00.4 UTC, just 9.5 seconds after the burst began. LOTIS obtained six images of the position centered on the Original GCN coordinates. 
LOTIS received the Final GCN coordinates of $(\alpha, \delta ; \mathrm{J} 2000.0)=(101.81,-7.81)$ at 5:18:09.7 UTC or approximately 138.8 seconds after the trigger. After slewing to the more accurate coordinates $(\sim 2.0 \mathrm{~s})$ LOTIS began imaging the Final GCN position at 5:18:09.7 UTC, approximately 140.8 seconds after the trigger. LOTIS obtained 49 images of the position centered on the Final GCN coordinates.

LOTIS received the more accurate LOCBURST coordinates of $(\alpha, \delta ; \mathrm{J} 2000.0)=$ (99.11, -8.23) approximately 18 minutes after the BATSE trigger. LOTIS obtained more than 450 images of the area around the LOCBURST GCN coordinates.

Figure 4.22 illustrates the LOTIS response time to GRB 990129. The solid line represents the BATSE gamma-ray light curve ( $>20 \mathrm{keV})$ with a duration of $\sim 220 \mathrm{~s}$. The grey shaded areas represent the $10.0 \mathrm{~s}$ integration times of the first seven LOTIS images. The first six cross hatched areas indicate LOTIS coverage of the Original GCN coordinates. The seventh LOTIS image was directed toward the Final GCN coordinates.

Figures 4.23 and 4.24 illustrate the LOTIS coverage of the final Huntsville error circle centered at $(\alpha, \delta ; \mathrm{J} 2000.0)=\left(94^{\circ} .93,-12.28\right)$, approximately $16^{\circ} .4$ from the Original GCN coordinates and 8.1 from the Final GCN coordinates. Figure 4.23 shows the coverage during the first six images and Figure 4.24 shows the coverage for the next 49 images. The inner and outer ellipses represent the BATSE $1 \sigma(0.35$ statistical error) and $3 \sigma$ error circles. The pseudo-rectangle represents the area covered by a single LOTIS camera (camera \#3). The upper left hand corner of the rectangle is the center of the $2 \times 2$ array of cameras. The area covered by the remaining three LOTIS cameras is not plotted. The narrow arc is the IPN annulus calculated using data from BATSE and the Ulysses GRB Monitor. The arc is center at $(\alpha, \delta ; \mathrm{J} 2000.0)=\left(162.669,-9^{\circ} .675\right)$, and has a radius and width of $64^{\circ} .179$ and $\pm 0^{\circ} 0393$, respectively. The probability content of the LOTIS coverage of GRB 990129 is $0.78 \%$ for the first six images and $86.31 \%$ for the remaining images. This probability does not include the IPN data. 


\section{GRB 990308}

On the evening of March $7^{\text {th }}-8^{\text {th }}$ LOTIS responded to 5 GCN Triggers: Trig. 7456 (Original), Trig. 7457 (Original), Trig. 7457 (Final), Trig. 7457 (Locburst), and Trig. 7457 (RXTE-ASM). Super-LOTIS was operating in debug mode on this night and therefore it did not respond to Trig. 7456. However a raster scan of the error box was manually started shortly after the trigger. The final Huntsville coordinates for Trig. 7456 had a statistical error of 5.1 centered at $(\alpha, \delta ; \mathrm{J} 2000.0)=$ $\left(48^{\circ} .13,+69.18\right)$, approximately $8^{\circ} 6$ from the Original coordinates. Neither LOTIS or Super-LOTIS sufficiently covered the error box of Trig. 7456 .

GRB 990308 (Trig. 7457) was detected by BATSE at 5:15:07.38 UTC (9:15:07.38 PST). LOTIS received the Original GCN coordinates of $(\alpha, \delta ; \mathrm{J} 2000.0)=(174: 35,+11: 33)$ at 5:15:12.4 UTC. After slewing to the coordinates ( $7.6 \mathrm{~s}$ ) LOTIS began imaging the Original GRB position at 5:15:20.0 UTC, just 12.6 seconds after the burst began. Five images were obtained at this location.

LOTIS received the more accurate Final GCN coordinates of $(\alpha, \delta ; \mathrm{J} 2000.0)=$ $(178.58,+5991)$ at 5:17:12.4 UTC. After slewing to the coordinates ( $6.7 \mathrm{~s})$ LOTIS began imaging the Final GCN position at 5:17:19.1 UTC, approximately 131.7 after the BATSE trigger. LOTIS obtained 40 images at this location. For the remainder of the evening LOTIS imaged the area centered on the Locburst GCN coordinates of $(\alpha, \delta ; \mathrm{J} 2000.0)=\left(185^{\circ} 82,+6^{\circ} 10\right)$ (Williams et al. 1999b).

During observations of the error box of Trig. 7456 Super-LOTIS received a second trigger for GRB 990308 (Trig. 7457). The system slewed to the Original GCN coordinates of $(\alpha, \delta ; \mathrm{J} 2000.0)=\left(174^{\circ} 35,11^{\circ} 33\right)$. During the debug mode SuperLOTIS was in a mode to observe one location without raster scanning. Therefore it was necessary to manually stop the observation and restart a raster scan. While the raster scan was being initiated Super-LOTIS received the LOCBURST coordinates of $(\alpha, \delta ; J 2000.0)=\left(185^{\circ} 82,6^{\circ} 10\right)$ which was set as the center of the raster scan.

GRB 990308 was also detected by the RXTE/ASM and the Ulysses GRB Monitor (Smith et al. 1999). The RXTE/ASM localized the burst to a narrow rect- 
angular region $10^{\circ} .0 \times 6 ! 6$ (full-width at $90 \%$ confidence) centered at $(\alpha, \delta ; \mathrm{J} 2000.0)=$ $(182.761832,+4.282319)$ and rotated $51^{\circ} 2$ east of north.

Figure 4.25 illustrates the LOTIS response time to GRB 990308. The solid line represents the BATSE gamma-ray light curve $(>20 \mathrm{keV})$ with a duration of $\sim 50 \mathrm{~s}$. The grey shaded areas represent the $10.0 \mathrm{~s}$ integration times of the first six LOTIS images. The cross hatched areas indicate LOTIS coverage of the Original GCN coordinates which did not cover the entire RXTE/ASM error box. The sixth image and all subsequent images covered the entire RXTE/ASM error box.

Figures 4.26 and 4.27 illustrate the LOTIS coverage of the final Huntsville error circle centered at $(\alpha, \delta ; \mathrm{J} 2000.0)=\left(189.16,+3^{\circ} 60\right)$, approximately 16.6 from the Original GCN coordinates and 10.8 from the Final GCN coordinates. Figure 4.26 shows the coverage during the first six images and Figure 4.27 shows the coverage for the remaining images. The inner and outer ellipses represent the BATSE $1 \sigma$ (1:9 statistical error) and $3 \sigma$ error circles. The pseudo-rectangles represent the area covered by the four LOTIS cameras. The narrow arc is the IPN annulus calculated using data from BATSE and the Ulysses GRB Monitor. The IPN arc is centered at $(\alpha, \delta ; \mathrm{J} 2000.0)=\left(154^{\circ} .07,-9.63\right)$ with a radius and width of 35.51 and \pm 0.23 respectively. The probability content of the LOTIS coverage of GRB 990308 is $1.14 \%$ for the first five images and $17.40 \%$ for the remaining images.

Four of the images ( $30 \mathrm{~s}$ integration) obtained during the Super-LOTIS raster scan covered the area of the RXTE/ASM error box. Image numbers 5, 7, 21, and 43 were obtained at $\mathrm{t}=1694 \mathrm{~s}, 1809 \mathrm{~s}, 2620 \mathrm{~s}$, and $3923 \mathrm{~s}$ after the BATSE trigger. Image 5 covered the entire error box consisting of the combined RXTE/ASM and IPN data. Figure 4.28 shows the coverage of the four Super-LOTIS images which covered the RXTE/ASM error box. Figure 4.29 is a close up of the crossing IPN annulus and the RXTE/ASM error boxes.

Analysis of the Super-LOTIS images failed to reveal any uncataloged objects within the error box to a limiting magnitude of $m=15.3 \pm 0.1$. Follow up observations by Schaefer et al. (1999) detected an optical counterpart at $(\alpha, \delta ; \mathrm{J} 2000.0)=$ $\left(12^{\mathrm{h}} 23^{\mathrm{m}} 11^{\mathrm{s}} .44 \pm 0.02,+06^{\circ} 44^{\prime} 05^{\prime \prime} 10 \pm 0^{\prime \prime} \cdot 17\right)$. The counterpart had magnitudes of $\mathrm{V}=$ 
$18.32 \pm 0.07$ at 196.8 minutes, $R=18.14 \pm 0.06$ at 200.6 minutes, $B=18.65 \pm 0.23$ at 204.5 minutes, and $R=18.22 \pm 0.05$ at 208.3 minutes after the burst. There were no other detections of this counterpart. The position of the optical counterpart is plotted as a star in Figure 4.29. For a full description of the LOTIS and Super-LOTIS data and the optical counterpart see Schaefer et al. (1999).

GRB 990316

GRB 990316 (Trig. 7475) was detected by BATSE on March 16, 1999 at 09:40:39.54 UTC (01:40:39.54 PST). LOTIS received the Original GCN coordinates of $(\alpha, \delta ; \mathrm{J} 2000.0)=(156: 08,-9.42)$ at 09:40:45.4 UTC, approximately 5.86 seconds after the trigger. After slewing to the coordinates ( $7.7 \mathrm{~s}$ ) LOTIS began imaging the Original GRB position at 09:40:53.1 UTC, just 13.6 seconds after the BATSE trigger. The first LOTIS image ( $30 \mathrm{~s}$ integration) was obtained simultaneous with the gamma-ray emission.

Figure 4.30 illustrates the LOTIS response time to GRB 990316. The solid line represents the BATSE gamma-ray light curve $(>20 \mathrm{keV})$ with a duration of $\sim 40 \mathrm{~s}$. The grey shaded area represents the integration times of the first three LOTIS images ( $30.0 \mathrm{~s}, 10.0 \mathrm{~s}$, and $10.0 \mathrm{~s})$. The following images had an integration time of $10.0 \mathrm{~s}$. LOTIS continued to image the area for the remaining 4 hours of darkness.

Figure 4.31 illustrates the LOTIS coverage of the final Huntsville error circle centered at $(\alpha, \delta ; \mathrm{J} 2000.0)=\left(152^{\circ} .09,-4^{\circ} 42\right)$, approximately 6.4 from the Original GCN coordinates. The inner and outer ellipses represent the BATSE $1 \sigma$ (0.3 statistical error) and $3 \sigma$ error circles. The narrow arc represents IPN annulus calculated using data from BATSE and the Konus GRB monitor. The annulus is centered at $(\alpha, \delta ; \mathrm{J} 2000.0)=(152.2023,-9.3594)$ and has a radius and width of 6.781 and \pm 0.3775 respectively. The pseudo-rectangles represent the area covered by the LOTIS cameras. The probability content of the LOTIS coverage of GRB 990316 is 95.38\%. This probability does not include the IPN data.

An optical transient was found in the first LOTIS image at $(\alpha, \delta ; \mathrm{J} 2000.0)=$ $(147.54,-4.95)$ which is inside the Huntsville $3 \sigma$ error box and just slightly $\left(49^{\prime \prime}\right)$ 
outside the initial IPN annulus Park et al. (1999a). The transient was not present in any later image. Although the transient has not been ruled out as a counterpart to GRB 990316 we give a low probability to the association because of its position relative the IPN annulus and the lack of any afterglow at the position at later times.

\section{GRB 990413}

GRB 990413 (Trig. 7518) was detected by BATSE on April 13, 1999 at 07:54:08.12 UTC (April 12, 23:54:08.12 PST). LOTIS received the Original GCN coordinates of $(\alpha, \delta ; \mathrm{J} 2000.0)=\left(180^{\circ} 68,-14: 74\right)(\mathrm{J} 2000.0)$ at 07:54:12.99 UTC, approximately 4.87 seconds after the trigger. After slewing to the coordinates $(\sim 8.1 \mathrm{~s})$ LOTIS began imaging the Original GRB position at 07:54:21.1 UTC, just 13.0 seconds after the BATSE trigger.

Figure 4.32 illustrates the LOTIS response time to GRB 990413. The solid line represents the BATSE gamma-ray light curve ( $>20 \mathrm{keV}$ ) with a duration of $\sim 15 \mathrm{~s}$. The grey shaded area represents the $10.0 \mathrm{~s}$ integration time of the first LOTIS image. The first LOTIS image was obtained simultaneous with the gamma-ray emission.

Figure 4.33 illustrates the LOTIS coverage of the final Huntsville error circle centered at $(\alpha, \delta ; \mathrm{J} 2000.0)=\left(179^{\circ} 86,-18^{\circ} .44\right)$, approximately 3.8 from the Original GCN coordinates. The inner and outer ellipses represent the BATSE $1 \sigma(1: 0$ statistical error) and $3 \sigma$ error circles. The narrow arc represents IPN annulus calculated using data from BATSE and the Konus GRB monitor. The annulus is centered at $(\alpha, \delta ; \mathrm{J} 2000.0)=\left(147.0779,-8^{\circ} .1387\right)$ and has a radius and width of $33^{\circ} .848$ and 0.117 respectively. The pseudo-rectangles represent the area covered by the LOTIS cameras. The probability content of the LOTIS coverage of GRB 990413 is $98.16 \%$. This probability does not include the IPN data.

GRB 990803

GRB 990803 (Trig. 7695) was detected by BATSE on August 3, 1999 at 06:29:35.92 UTC (August 2, 23:29:35.92 PST). LOTIS received the Original GCN coordinates of $(\alpha, \delta ; \mathrm{J} 2000.0)=\left(258^{\circ} 17,+0^{\circ} .18\right)$ at $06: 29: 40.67$ UTC, approximately 
4.75 seconds after the trigger. After slewing to the coordinates ( $\sim 10.2 \mathrm{~s}$ ) LOTIS began imaging the Original GRB position at 06:29:50.9 UTC, just 15.0 seconds after the BATSE trigger.

LOTIS received the updated Final GCN coordinates of $(\alpha, \delta ; J 2000.0)=\left(259^{\circ} 74,-1^{\circ} .73\right)$ at 06:30:51.57 UTC, approximately 75.65 seconds after the BATSE trigger. LOTIS continued to image the Final location until the Locburst coordinates of $(\alpha, \delta ; \mathrm{J} 2000.0)=$ $(258.65,-2.33)$ arrived at $06: 53: 55.13$, approximately 1459.21 seconds after the trigger.

Figure 4.34 illustrates the LOTIS response time to GRB 990803. The solid line represents the BATSE gamma-ray light curve $(>20 \mathrm{keV})$ with a duration of $\sim 25 \mathrm{~s}$. The grey shaded area represents the $10.0 \mathrm{~s}$ integration time of the first LOTIS images. The first LOTIS image was obtained during gamma-ray emission.

The LOTIS coverage plot of GRB 990803 is not yet available. The final Huntsville error circle is centered at $(\alpha, \delta ; \mathrm{J} 2000.0)=\left(258^{\circ} .65,-2.00\right)$, approximately 2.2 from the Original GCN coordinates. The LOTIS camera \#3 offset malfunctioned prior to the burst yielding an image with a zero background level and only the brightest stars visible. Therefore data from the area covered by camera \#3 is unusable. The final Huntsville statistical error for GRB 990803 was 0:63. There were two IPN arcs available for this burst which crossed resulting in a reduced error box. LOTIS covered the entire intersection but part of the area was in camera \#3. The probability content of the LOTIS coverage of GRB 990803 (not) including camera \# 3 is $92.60 \%$ $(89.23 \%)$.

\section{GRB 990918}

GRB 990918 (Trig. 7770) was detected by BATSE on September 18, 1999 at 05:33:14.87 UTC (September 17, 22:33:14.87 PST). LOTIS received the Original GCN coordinates of $(\alpha, \delta ; \mathrm{J} 2000.0)=\left(267.25,+8^{\circ} .76\right)$ at 05:33:19.33 UTC, approximately 4.46 seconds after the trigger. After slewing to the coordinates ( $\sim 3.9 \mathrm{~s}$ ) LOTIS began imaging the Original GRB position at 05:33:23.2 UTC, just 8.3 seconds after the BATSE trigger. LOTIS received the updated Final GCN coordinates 
at 05:35:13.8 UTC of $(\alpha, \delta ; \mathrm{J} 2000.0)=\left(265^{\circ} .39,+8^{\circ} .93\right)$, approximately 118.9 seconds after the trigger. LOTIS continued to image the Final location until the Locburst coordinates of $(\alpha, \delta ; \mathrm{J} 2000.0)=\left(262^{\circ} .88,+2.10\right)$ arrived at 05:55:24.2, approximately 1329.3 seconds after the trigger.

Figure 4.35 illustrates the LOTIS response time to GRB 990918. The solid line represents the BATSE gamma-ray light curve $(>20 \mathrm{keV})$ with a duration of $\sim 6.5 \mathrm{~s}$. The grey shaded area represents the $10.0 \mathrm{~s}$ integration time of the first LOTIS images. The first LOTIS image was obtained approximately 2.0 seconds after the gamma-ray emission ceased.

Figure 4.36 illustrates the LOTIS coverage of the final Huntsville error circle centered at $(\alpha, \delta ; \mathrm{J} 2000.0)=(262.08,+1.94)$, approximately 8.54 from the Original coordinates. The inner and outer ellipses represent the BATSE $1 \sigma(1.5$ statistical error) and $3 \sigma$ error circles. The narrow arc represents IPN annulus calculated using data from BATSE and the Konus GRB monitor. The annulus is centered at $(\alpha, \delta ; \mathrm{J} 2000.0)=\left(338^{\circ} .31,+15^{\circ} .05\right)$ and has a radius and width of $74^{\circ} .57$ and 0.02 respectively. The pseudo-rectangle represents the area covered by a single LOTIS cameras (camera \#1). The area covered by the other LOTIS cameras is not plotted. The probability content of the LOTIS coverage of GRB 990918 is $81.40 \%$. This probability does not include the IPN data. 
GRB $961017(\mathrm{E}>20 \mathrm{keV})$
(BATSE Trig. 5634)

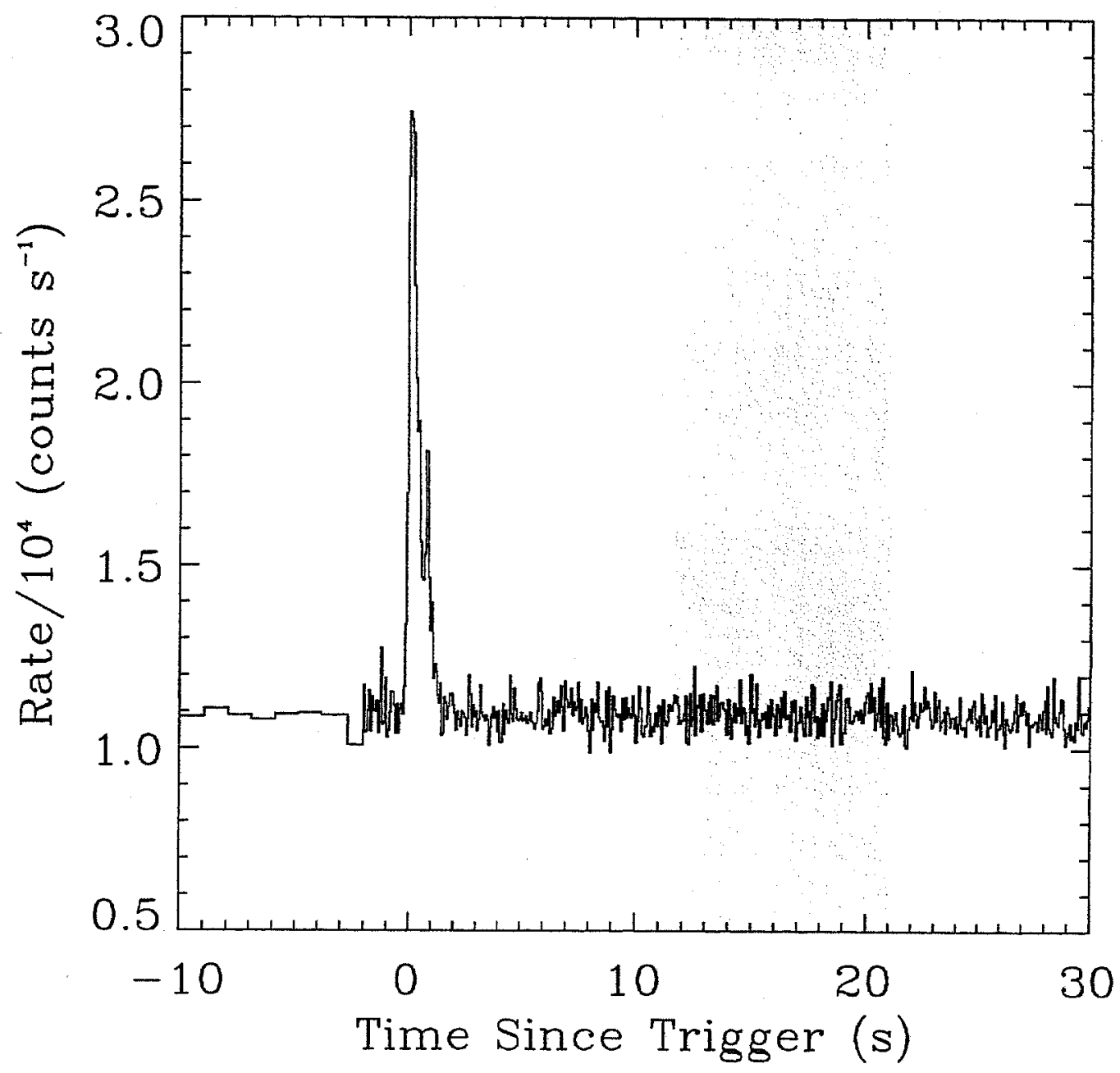

Figure 4.6 The BATSE gamma-ray light curve for GRB 961017. The grey shaded area represents the integration time of the first LOTIS image. 


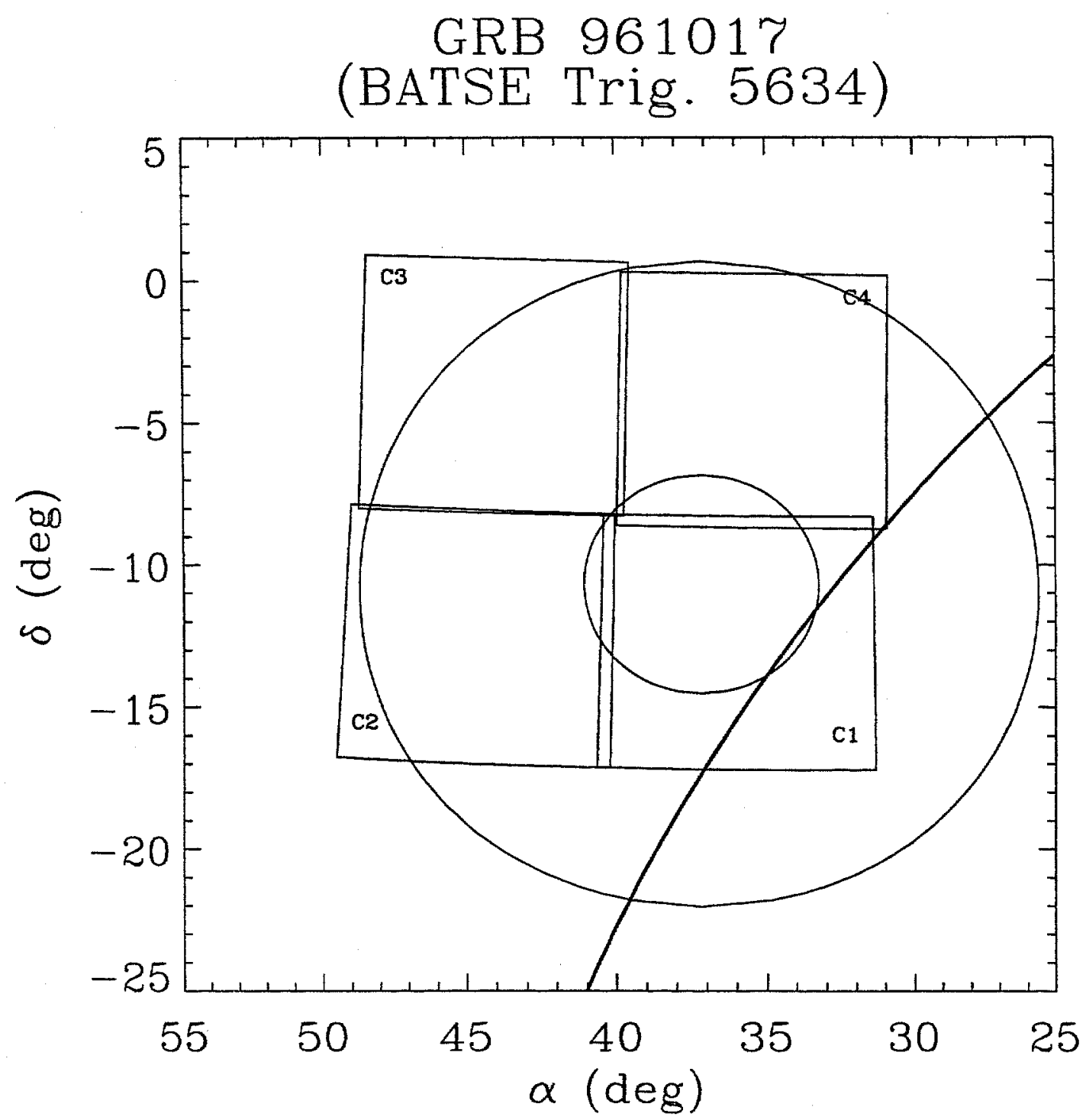

Figure 4.7 LOTIS coverage of GRB 961017. The pseudo-squares represent the area covered by the four LOTIS cameras. The circles represent the BATSE $1 \sigma$ and $3 \sigma$ error circles. The narrow arc is the IPN annulus. 


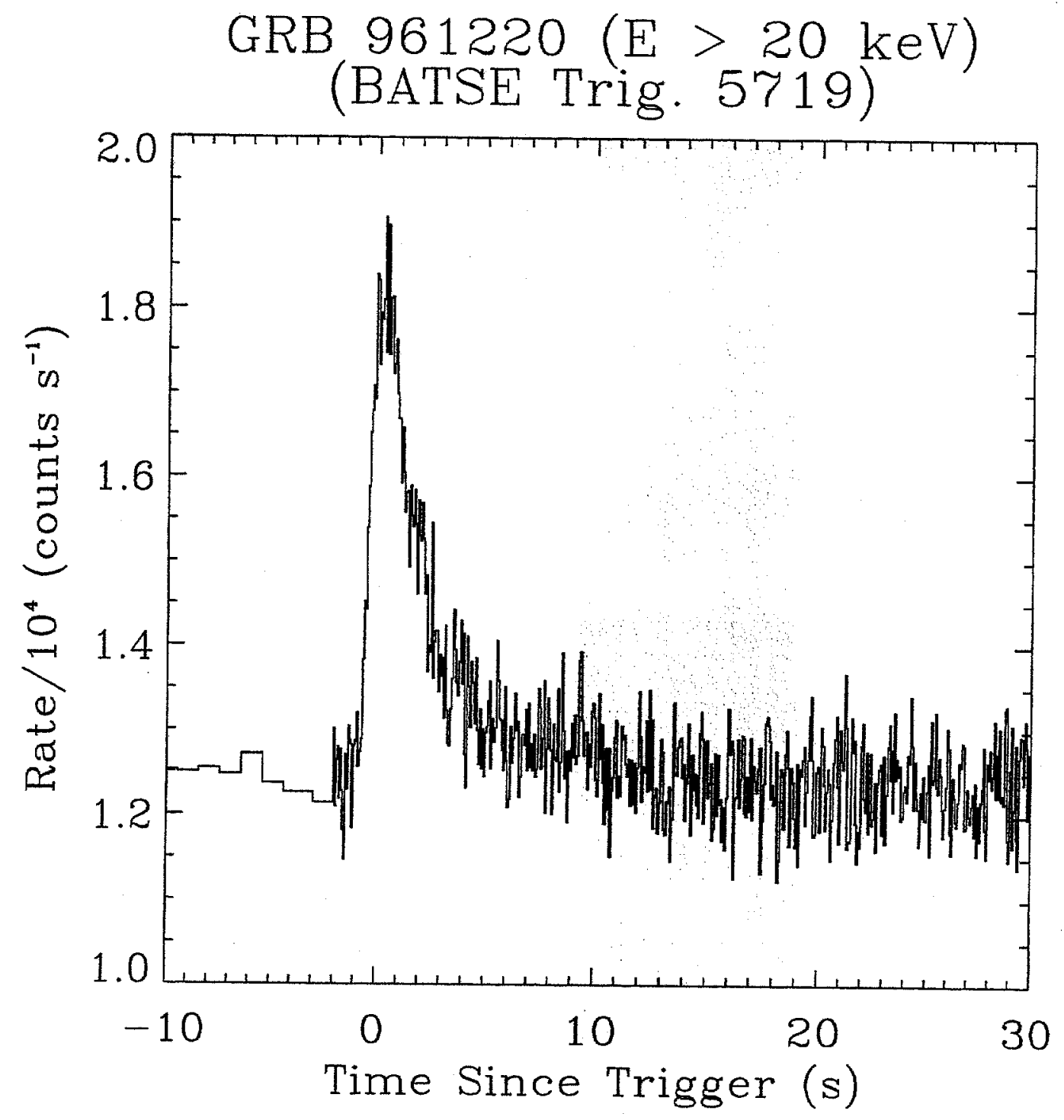

Figure 4.8 The BATSE gamma-ray light curve for GRB 961220. The grey shaded area represents the integration time of the first LOTIS image. 


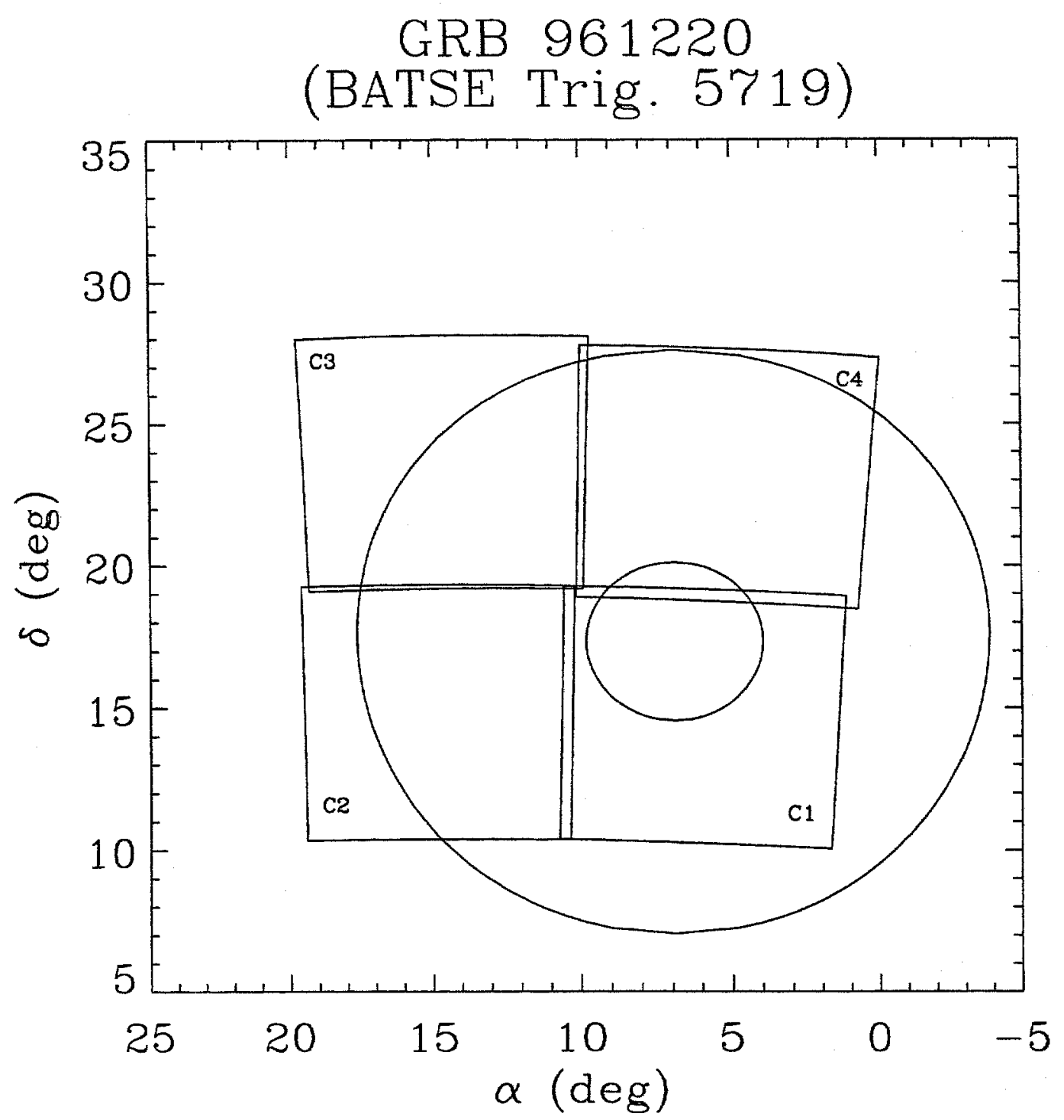

Figure 4.9 LOTIS coverage of GRB 961220. The pseudo-squares represent the area covered by the four LOTIS cameras. The circles represent the BATSE $1 \sigma$ and $3 \sigma$ error circles. 


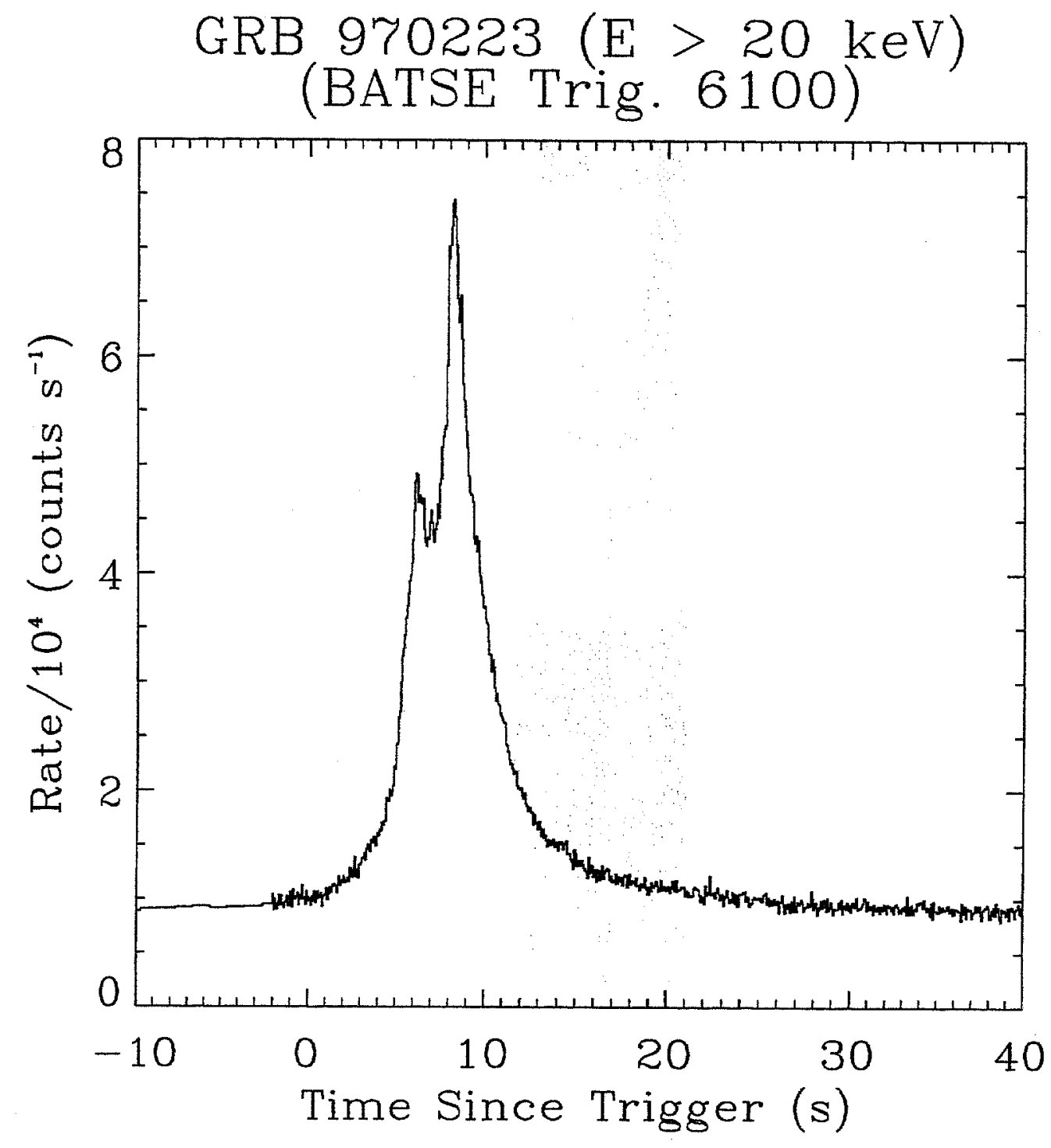

Figure 4.10 The BATSE gamma-ray light curve for GRB 970223. The grey shaded area represents the integration time of the first LOTIS image. 


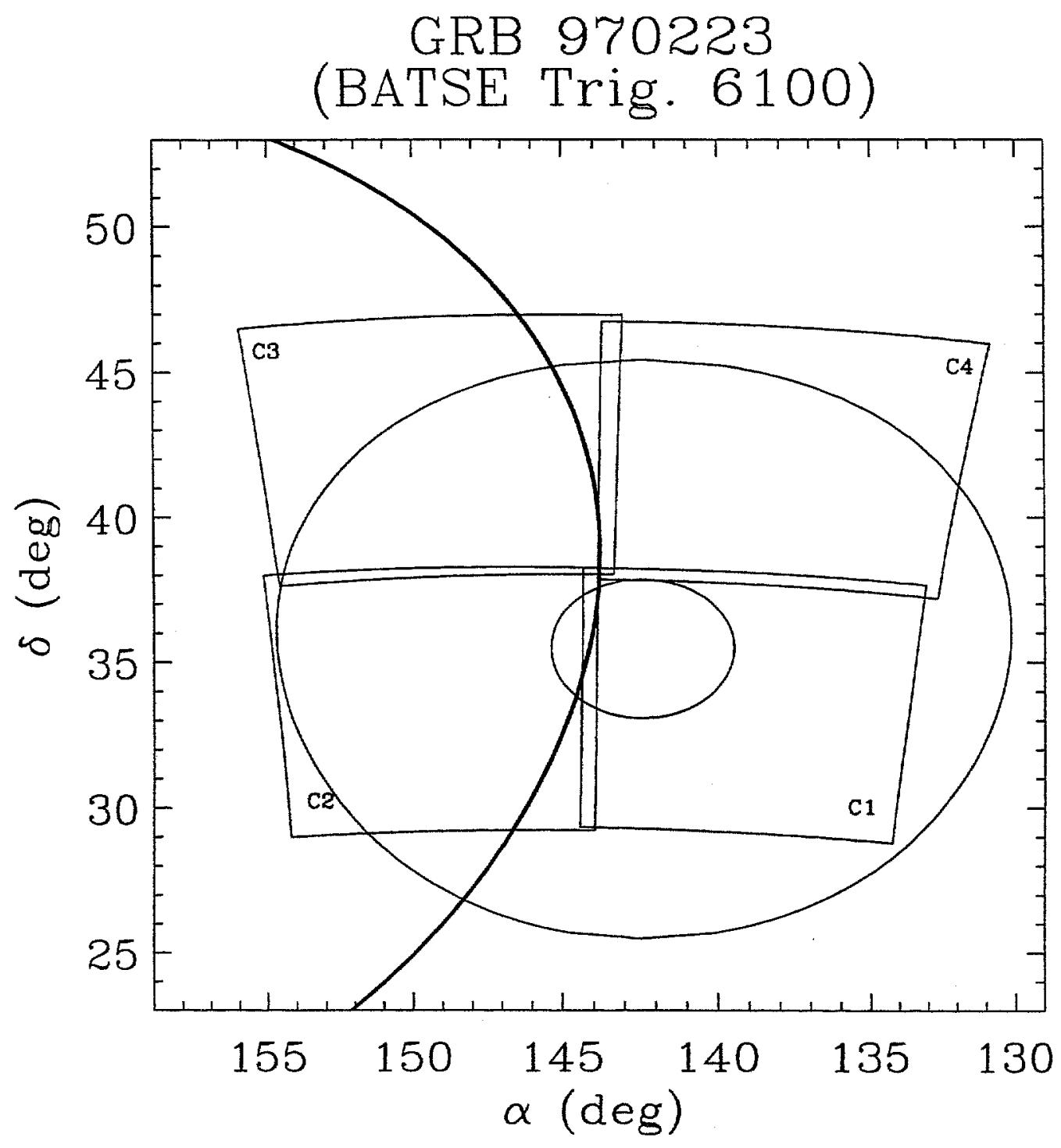

Figure 4.11 LOTIS coverage of GRB 970223. The pseudo-squares represent the area covered by the four LOTIS cameras. The circles represent the BATSE $1 \sigma$ and $3 \sigma$ error circles. The narrow arc is the IPN annulus. 


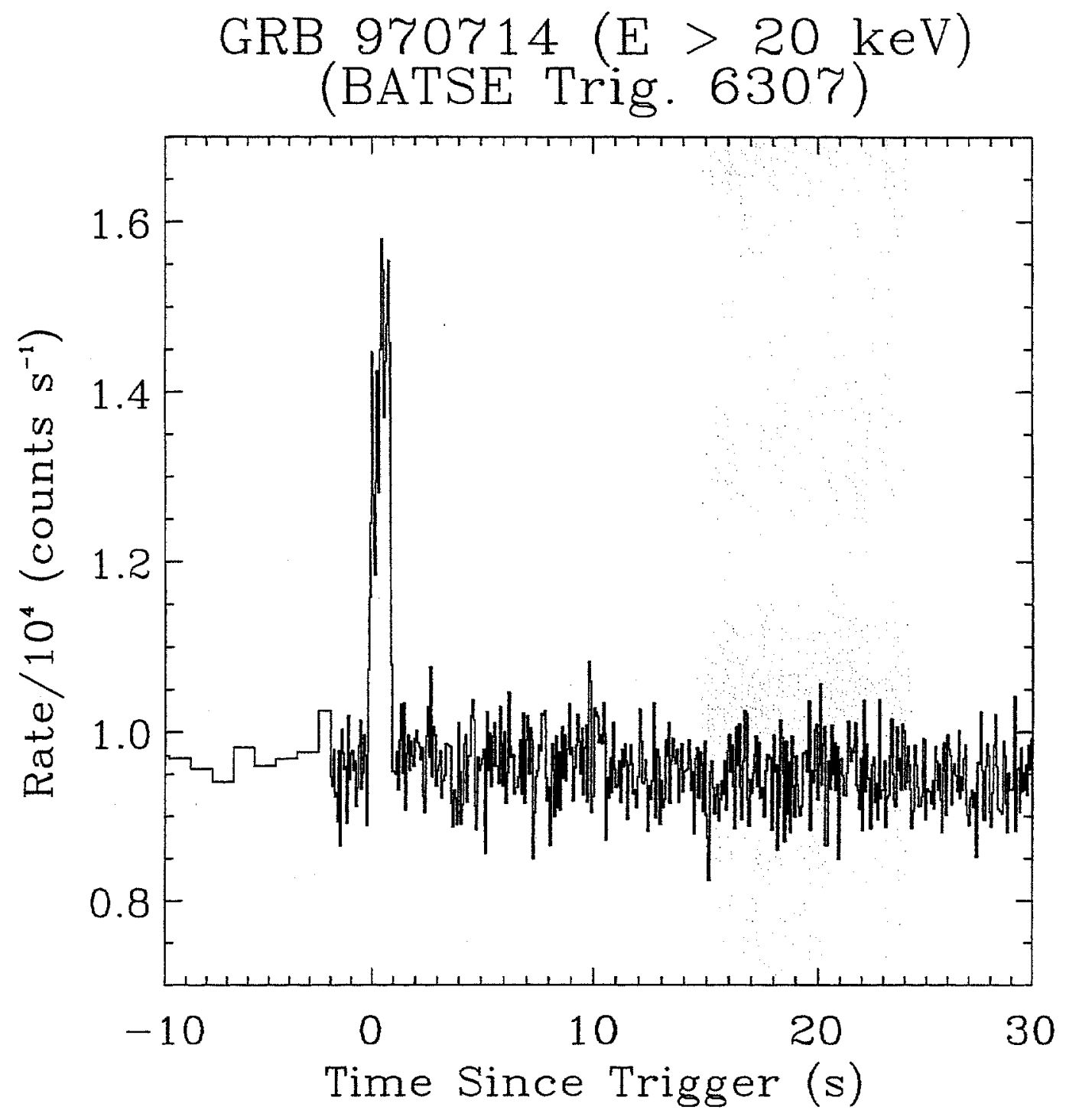

Figure 4.12 The BATSE gamma-ray light curve for GRB 970714. The grey shaded area represents the integration time of the first LOTIS image. 


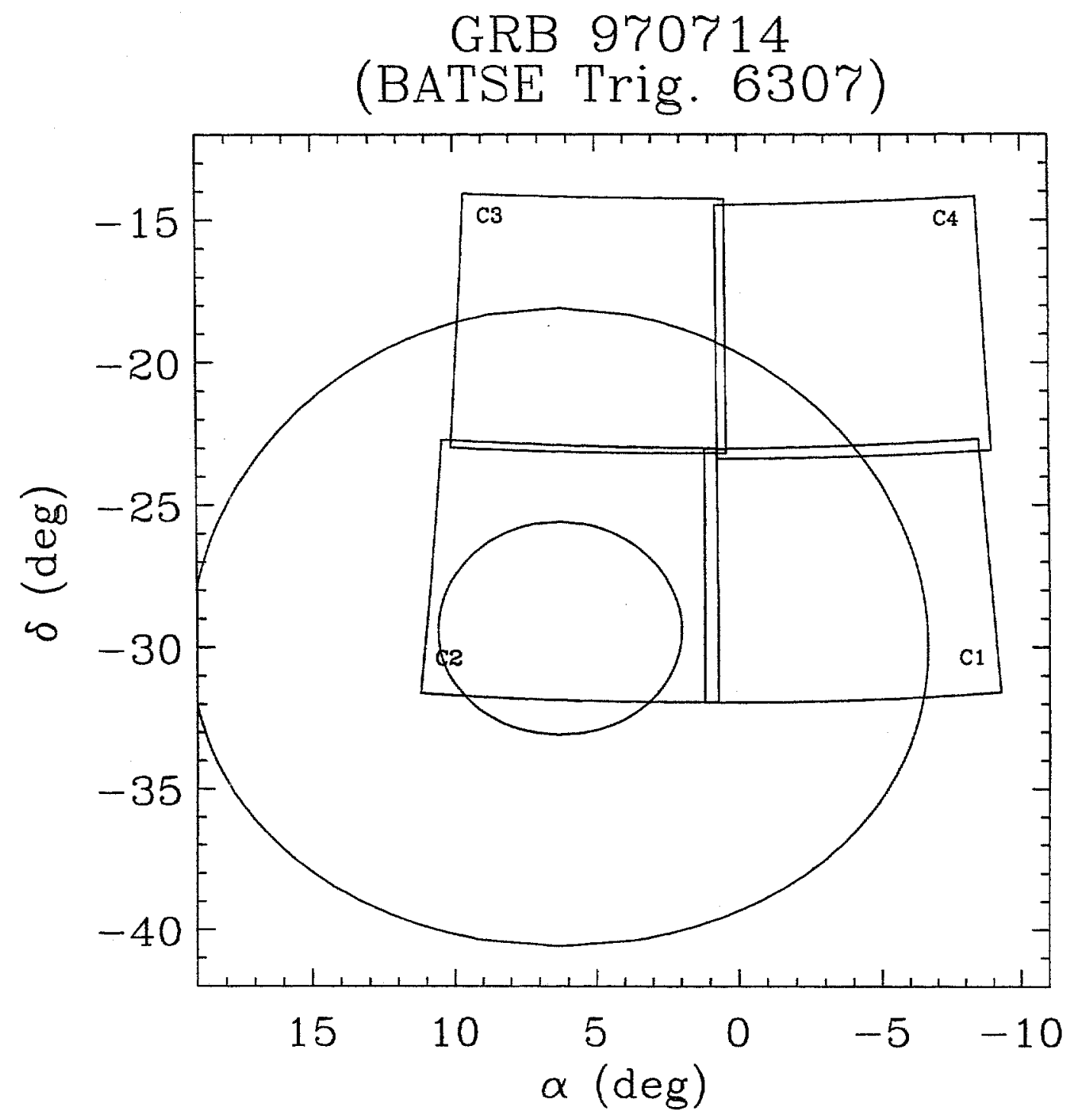

Figure 4.13 LOTIS coverage of GRB 970714. The pseudo-squares represent the area covered by the four LOTIS cameras. The circles represent the BATSE $1 \sigma$ and $3 \sigma$ error circles. 


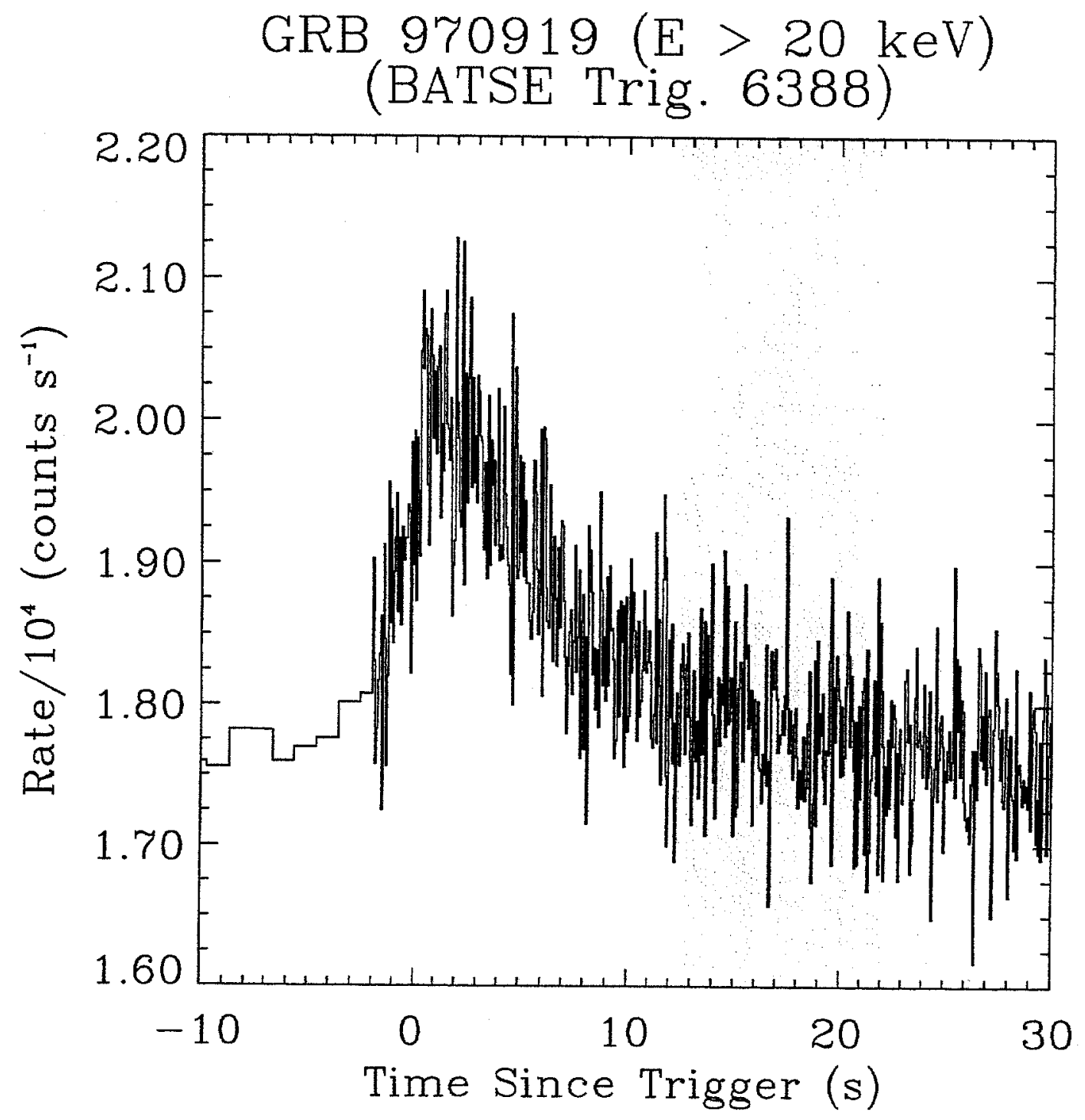

Figure 4.14 The BATSE gamma-ray light curve for GRB 970919. The grey shaded area represents the integration time of the first LOTIS image. 


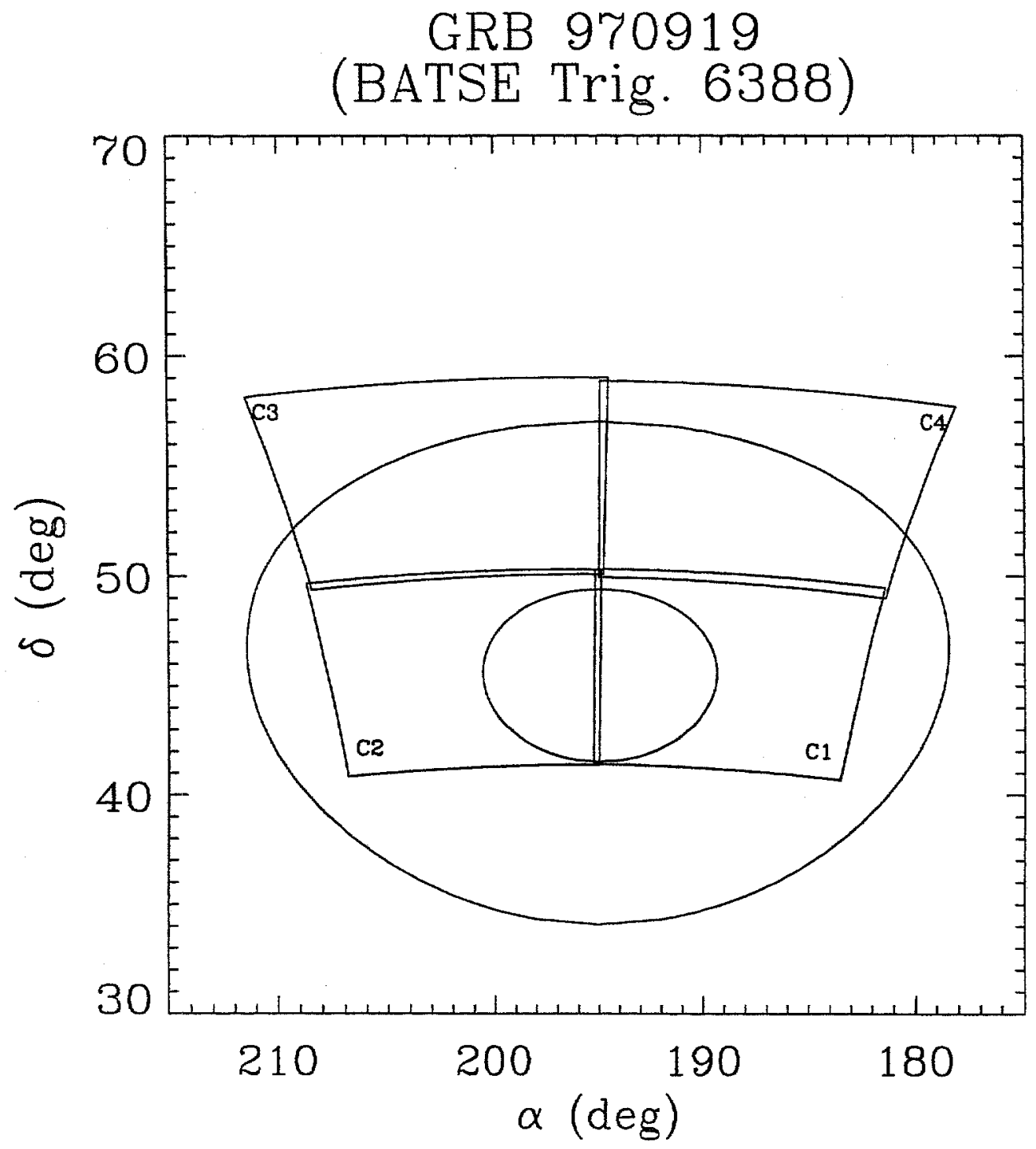

Figure 4.15 LOTIS coverage of GRB 970919. The pseudo-squares represent the area covered by the four LOTIS cameras. The circles represent the BATSE $1 \sigma$ and $3 \sigma$ error circles. 


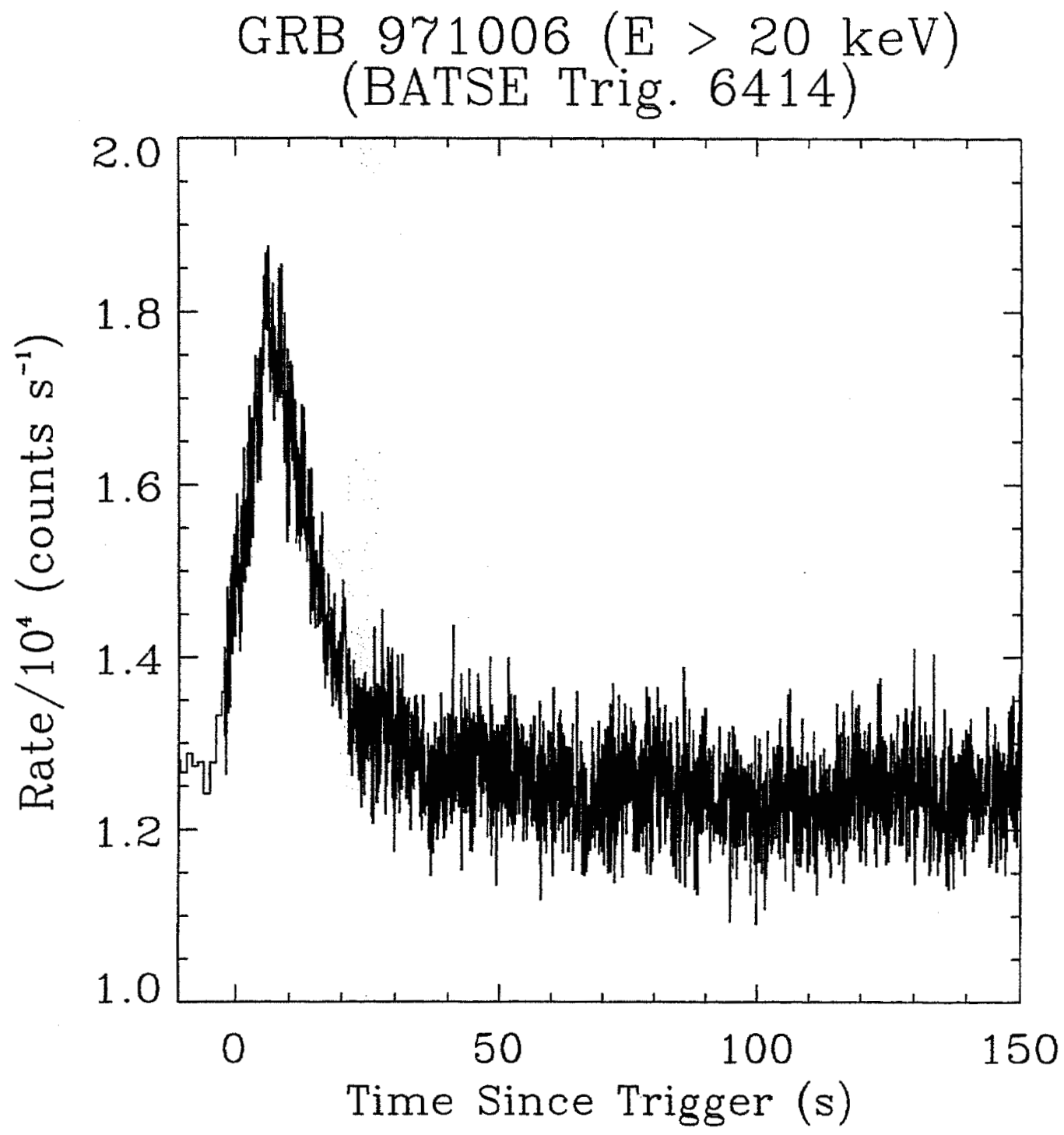

Figure 4.16 The BATSE gamma-ray light curve of GRB 971006 . The grey shaded area represents the integration time of the first LOTIS image. 


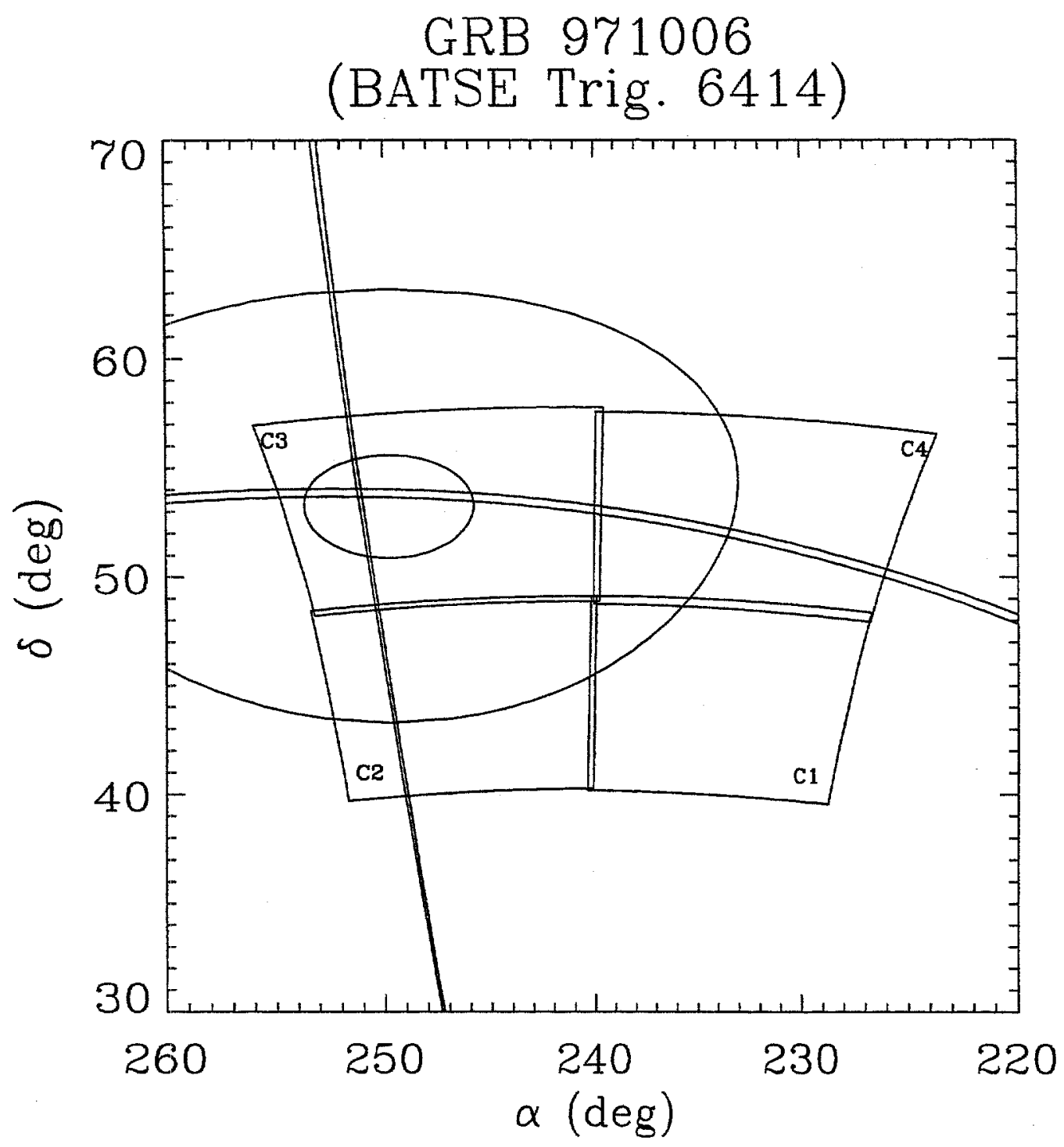

Figure 4.17 LOTIS coverage of GRB 971006. The pseudo-squares represent the area covered by the four LOTIS cameras. The circles represent the BATSE $1 \sigma$ and $3 \sigma$ error circles. The two narrow arcs are IPN annuli. 


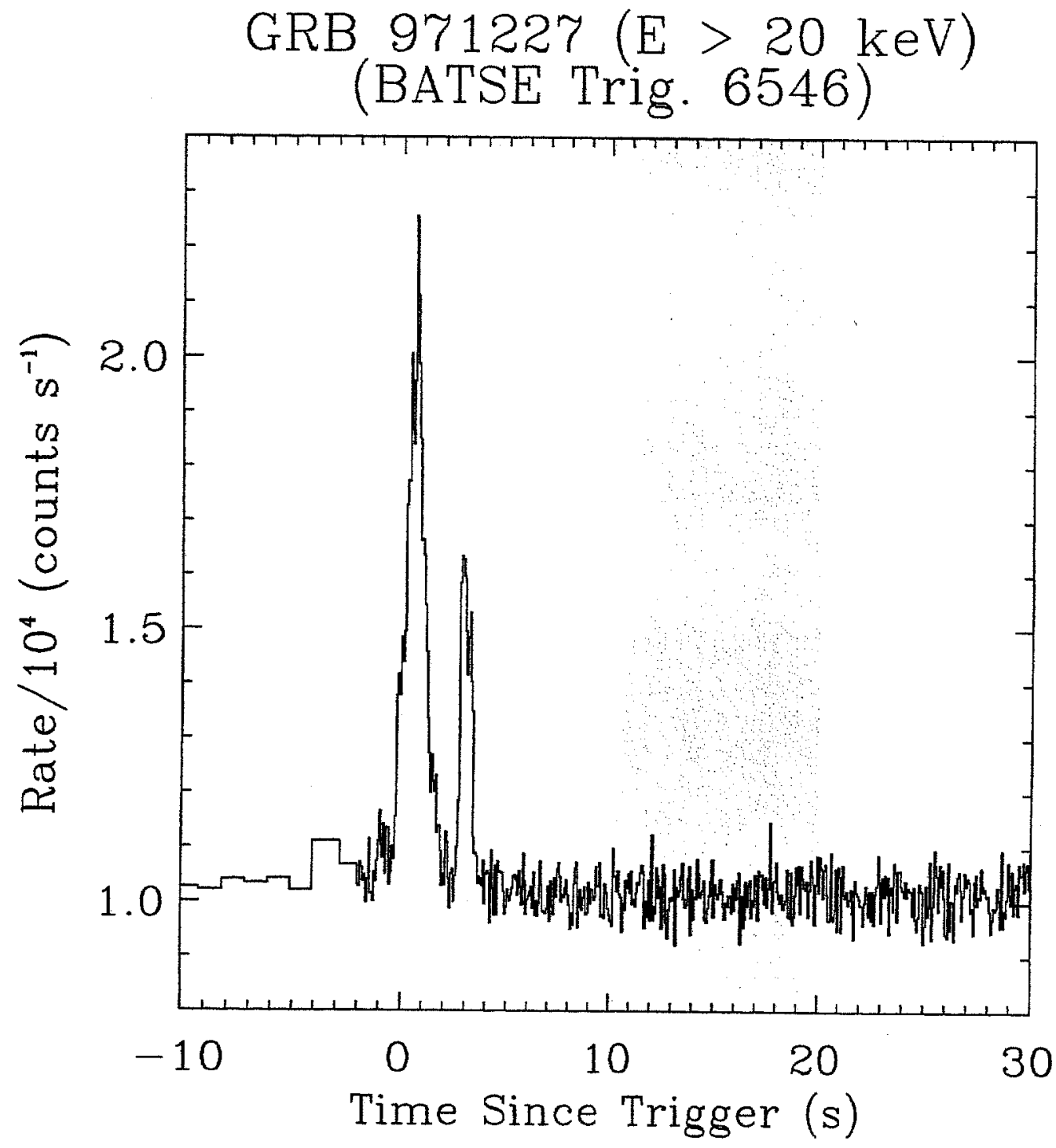

Figure 4.18 The BATSE gamma-ray light curve of GRB 971227. The grey shaded area represents the integration time of the first LOTIS image. 


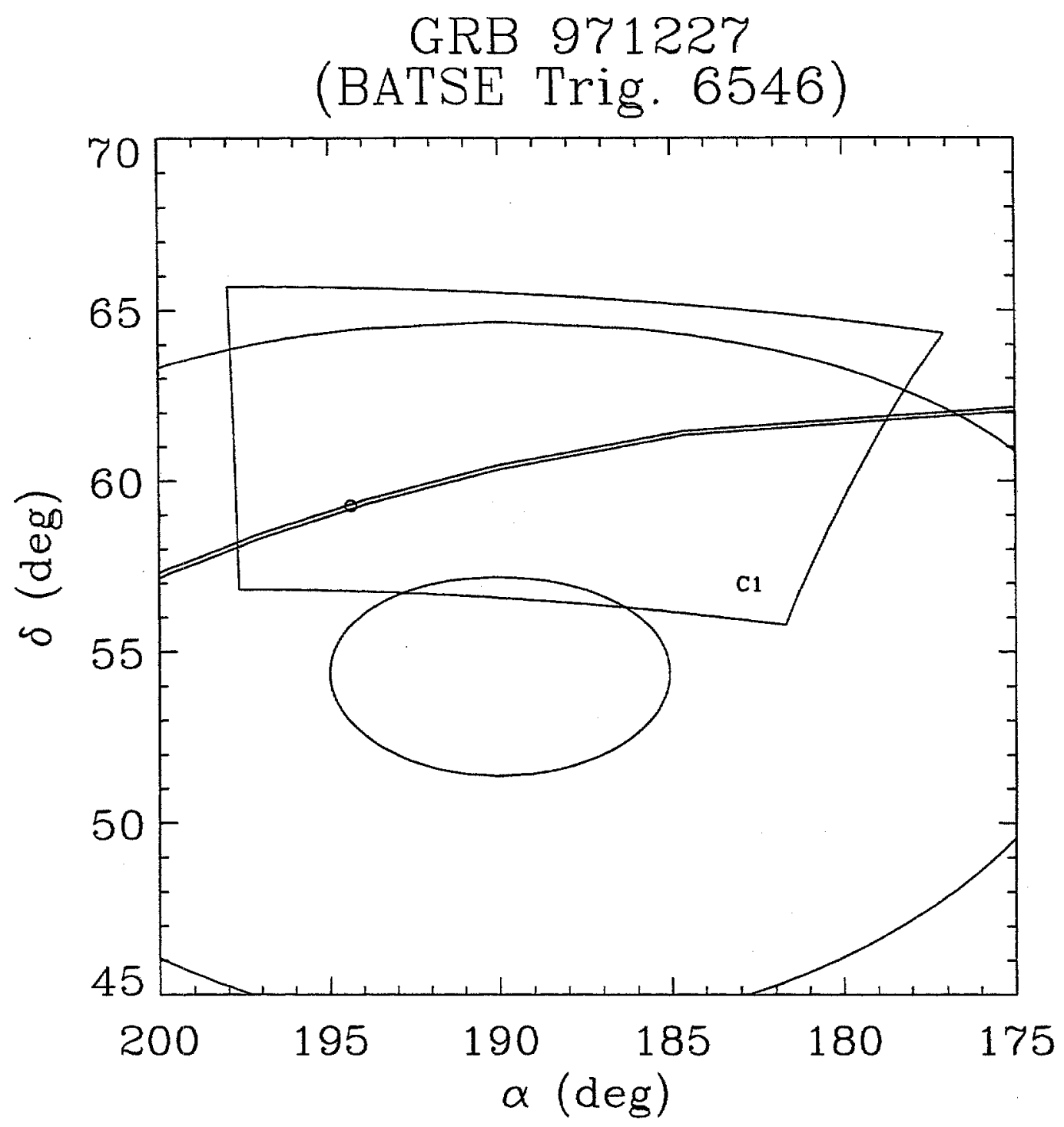

Figure 4.19 LOTIS coverage of GRB 971227. The pseudo-square represents the area covered by LOTIS camera \#1. The large circles represent the BATSE $1 \sigma$ and $3 \sigma$ error circles. The narrow arc is the IPN annulus and the small circle is the SAX/WFC error box. 


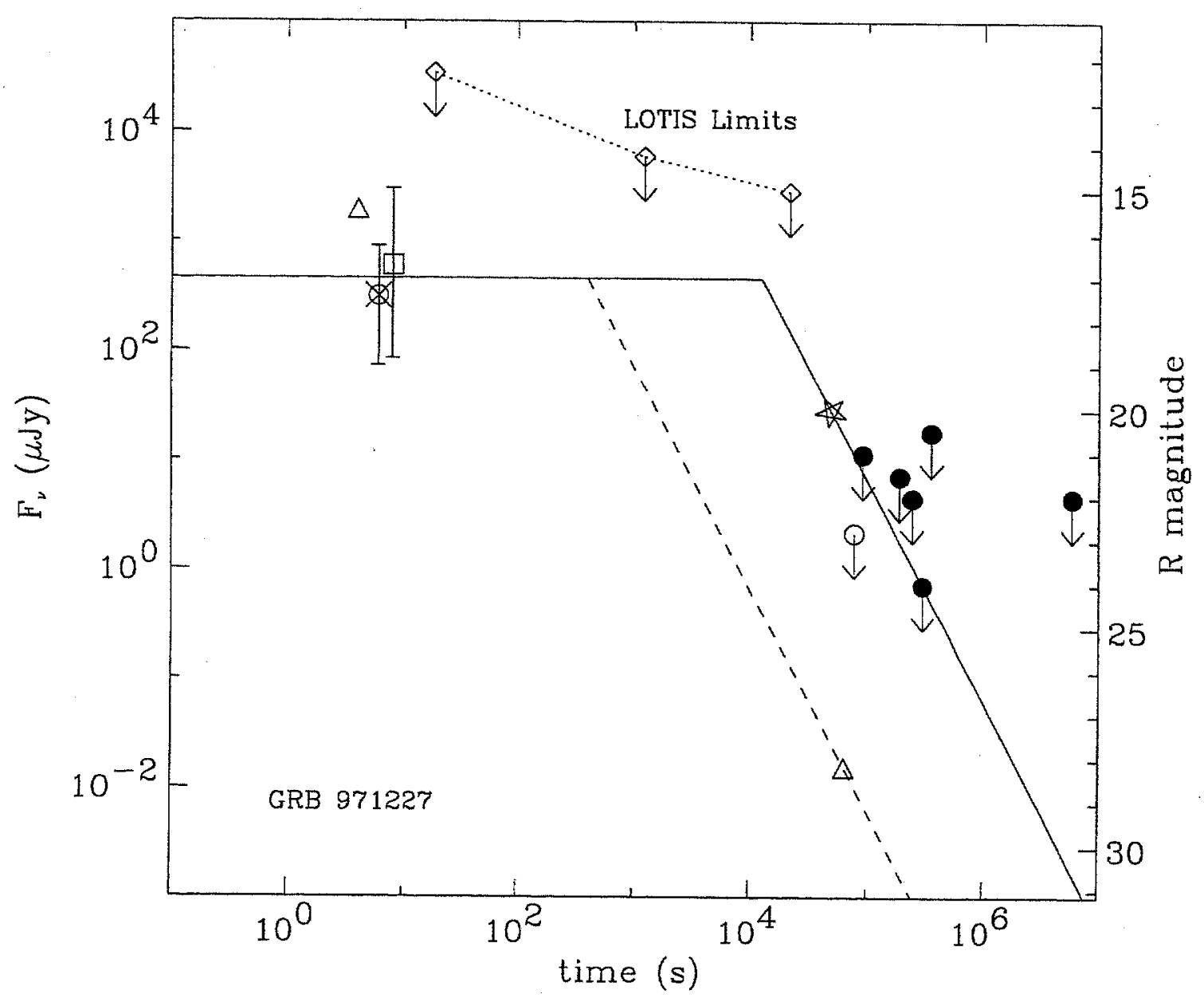

Figure 4.20 Broadband light curve of GRB 971227. The diamonds are the LOTIS upper limits. The crossed circle and square are the gamma-ray $(100 \mathrm{keV})$ and X-ray $(5 \mathrm{keV})$ flux densities derived from BATSE data. The triangles are X-ray flux densities derived from BeppoSAX data. The star represents the possible optical afterglow. Rband upper limits are plotted as filled circles. The open circle is an upper limit which did not cover the area of the suspected optical afterglow. The solid line illustrates the predictions from a simple fireball model (Williams et al. 1999a). 


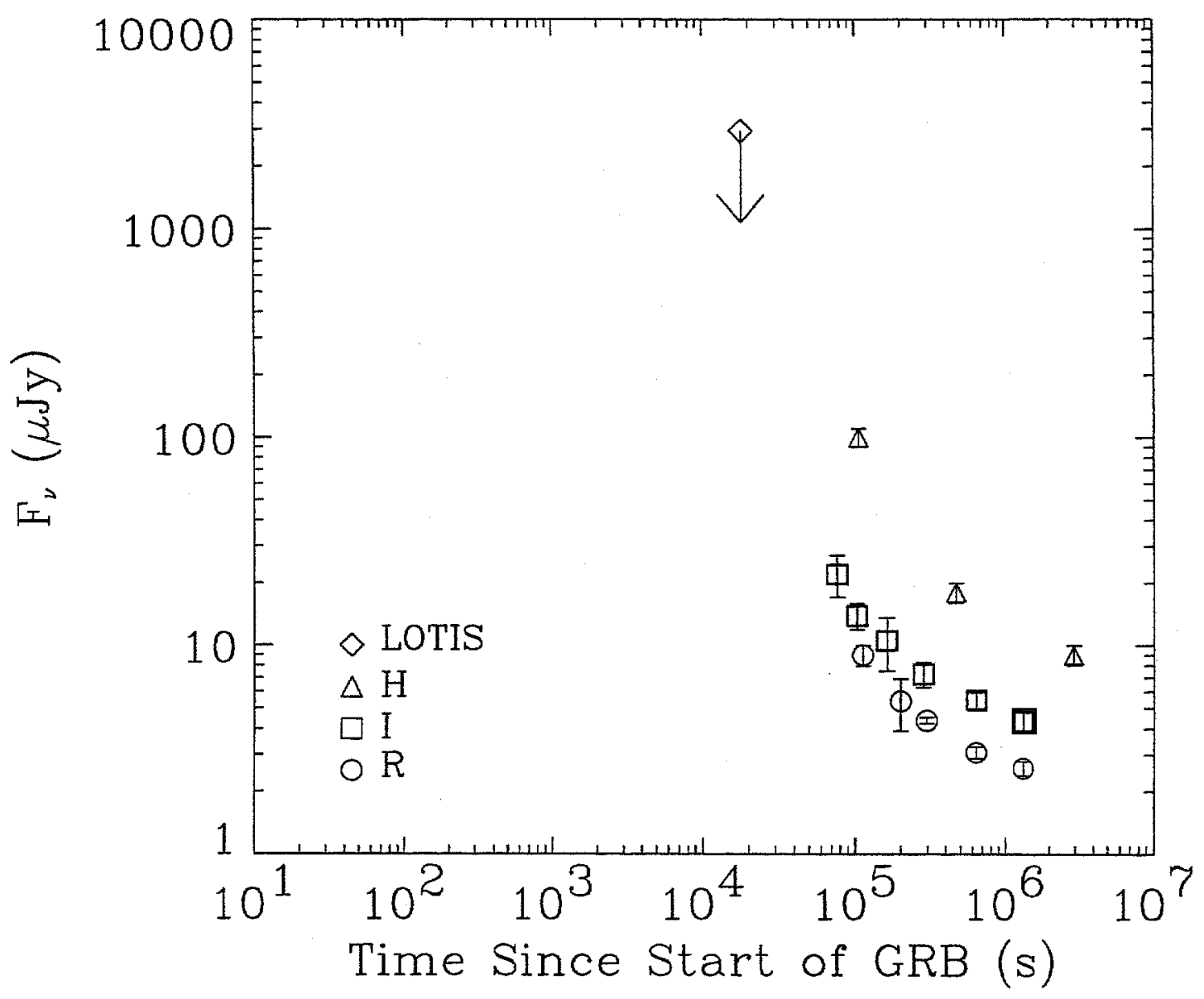

Figure 4.21 Optic afterglow light curve of GRB 980703. The diamond represents the LOTIS upper limit. The circles, squares, and triangles are the combined H, I, and R band detections (Park et al. 1999b). 


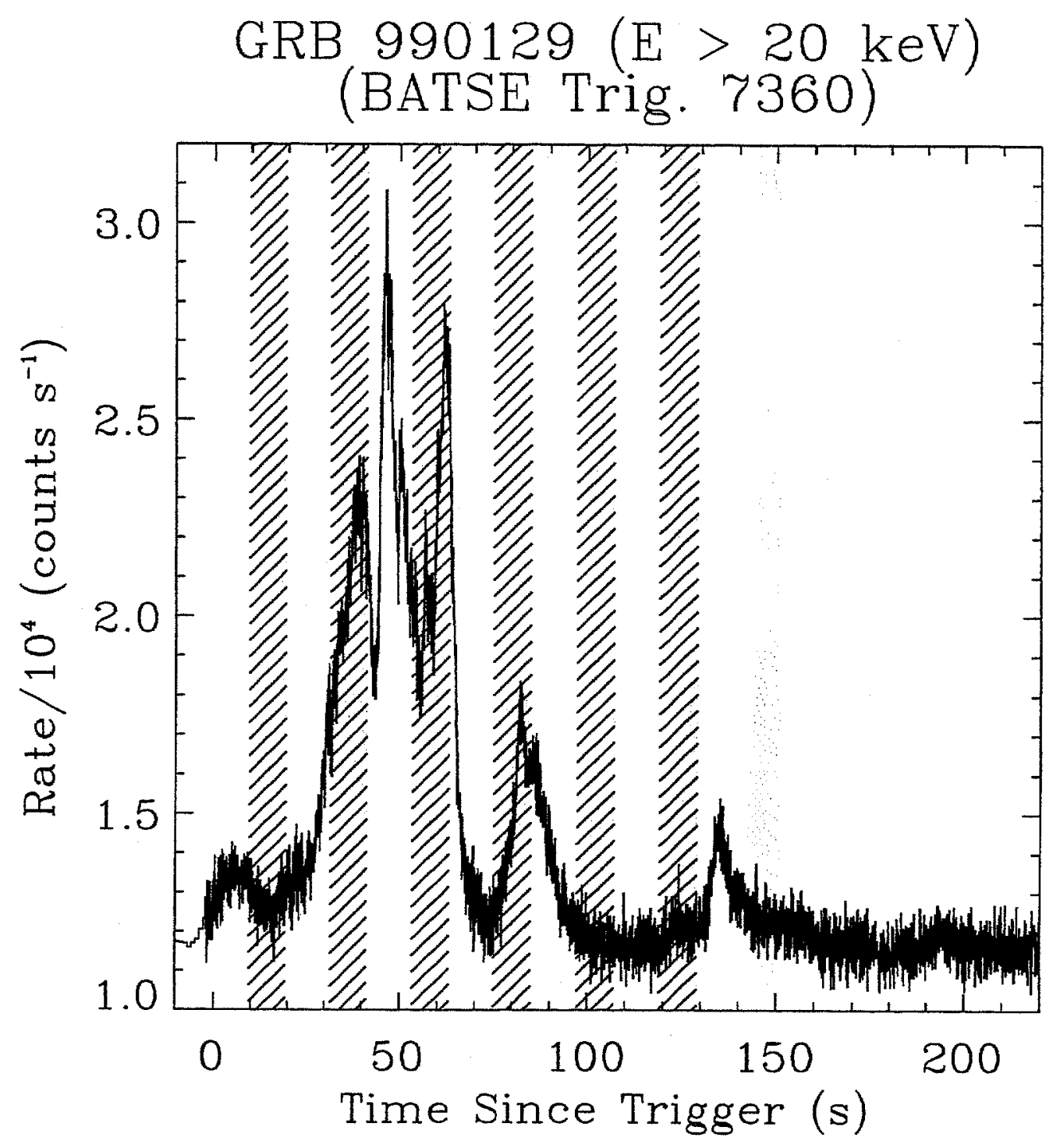

Figure 4.22 The BATSE gamma-ray light curve of GRB 990129. The grey shaded areas represent the integration times of the first seven LOTIS images. The crosshatched areas indicate insufficient coverage of the final Huntsville coordinates. 


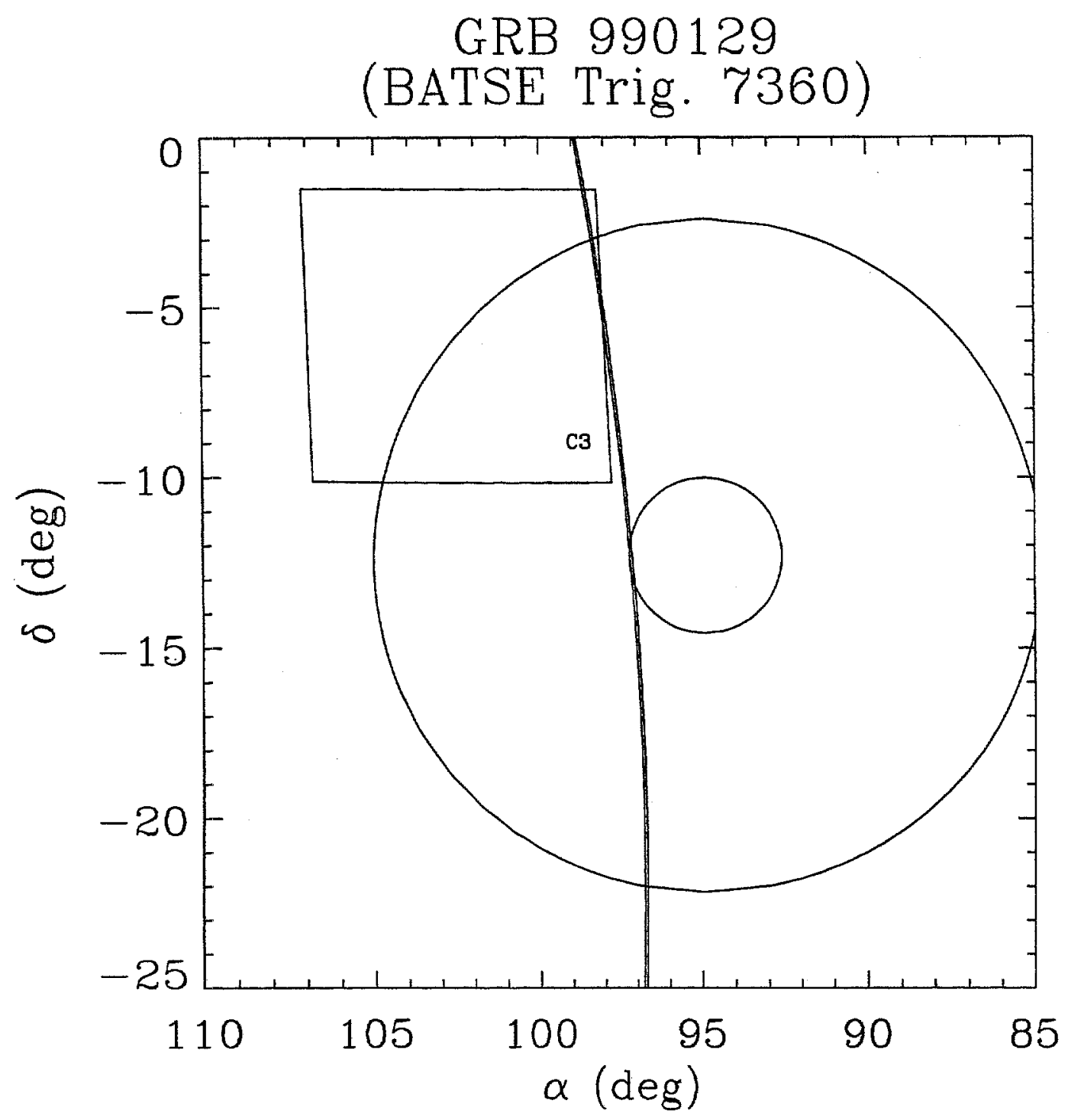

Figure 4.23 LOTIS coverage of GRB 990129; first image. The pseudo-square represents the area covered by LOTIS camera \#3. The large circles represent the BATSE $1 \sigma$ and $3 \sigma$ error circles. The narrow arc is the IPN annulus. 


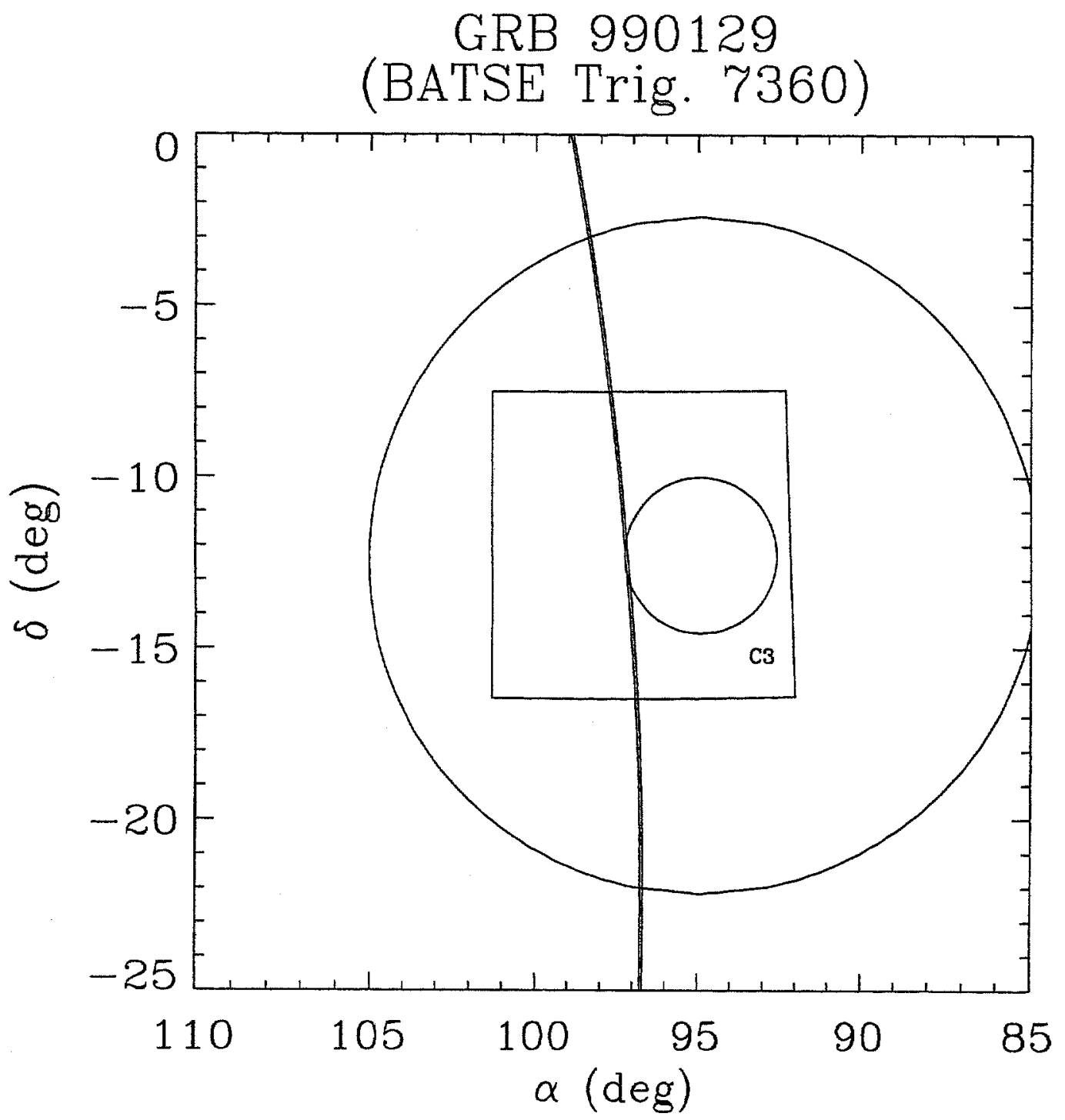

Figure 4.24 LOTIS coverage of GRB 990129; seventh image. The pseudo-square represents the area covered by LOTIS camera \#3. The large circles represent the BATSE $1 \sigma$ and $3 \sigma$ error circles. The narrow arc is the IPN annulus. 


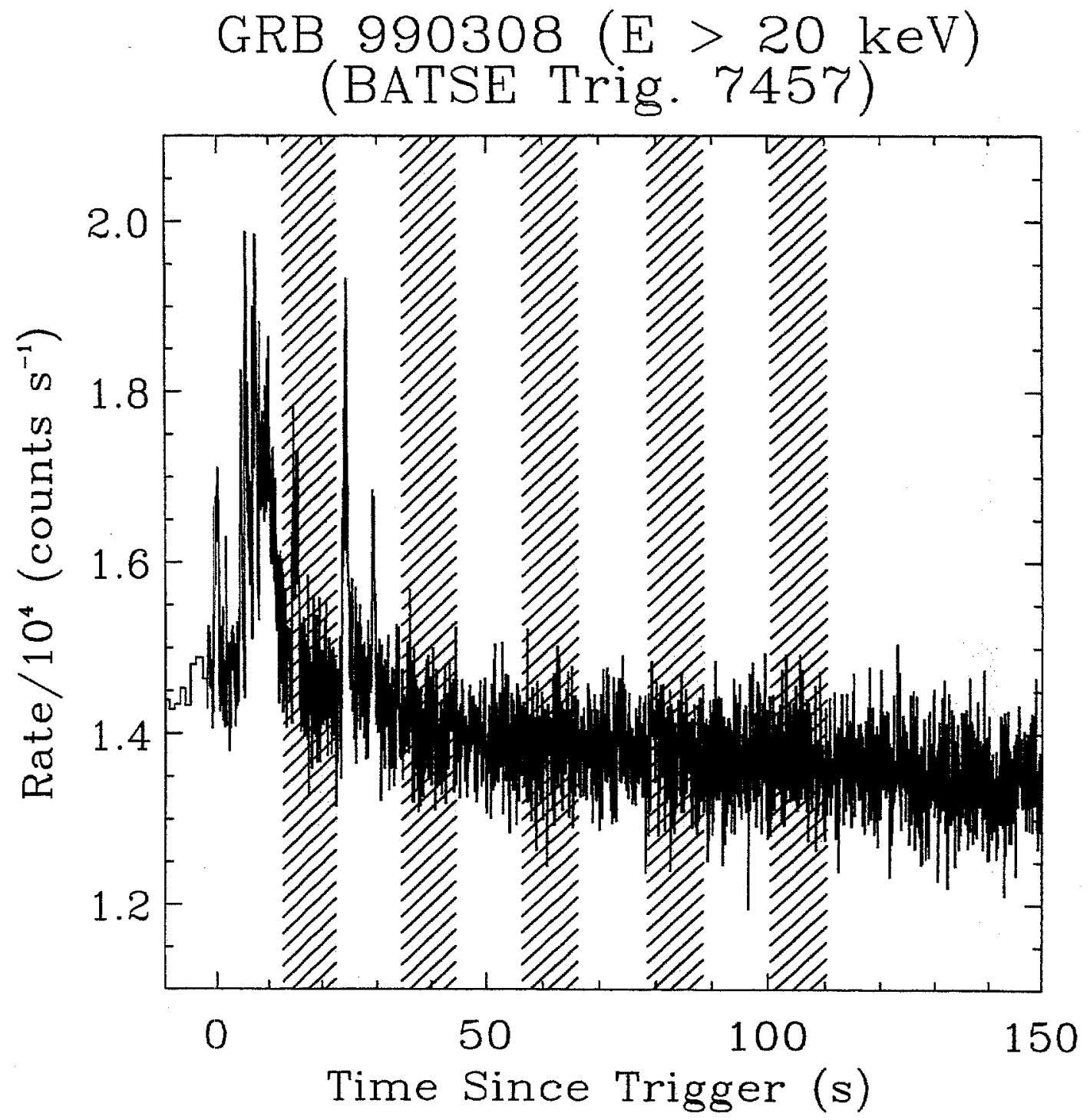

Figure 4.25 The BATSE gamma-ray light curve of GRB 990308. The grey shaded areas represent the integration times of the first six LOTIS images. The crosshatched areas indicate insufficient coverage of the final Huntsville coordinates. 


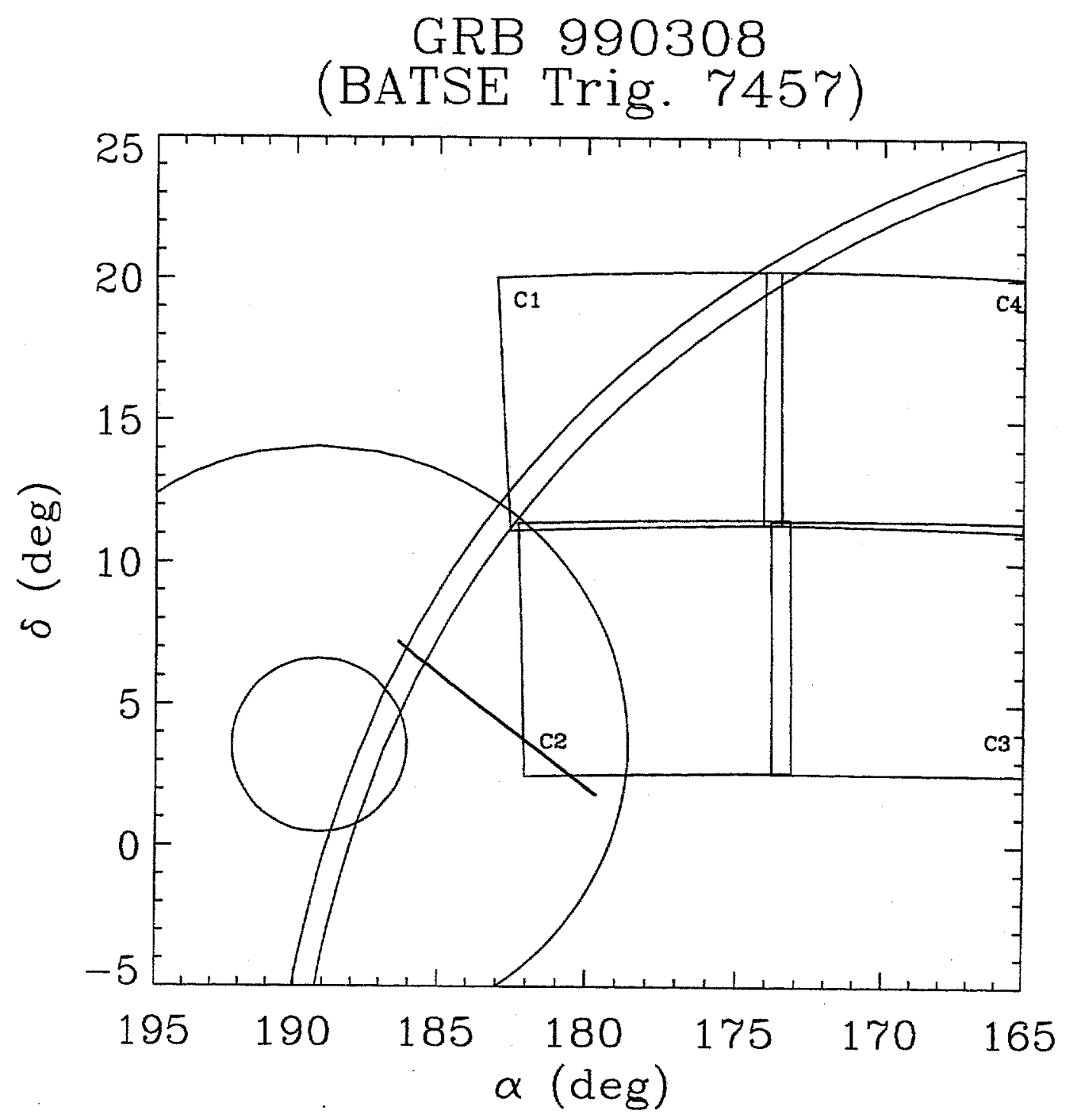

Figure 4.26 LOTIS coverage of GRB 990308; first image. The pseudo-squares represent the area covered by the four LOTIS cameras. The large circles represent the BATSE $1 \sigma$ and $3 \sigma$ error circles. The narrow arc is the IPN annulus. The long narrow box is the RXTE/ASM error box 


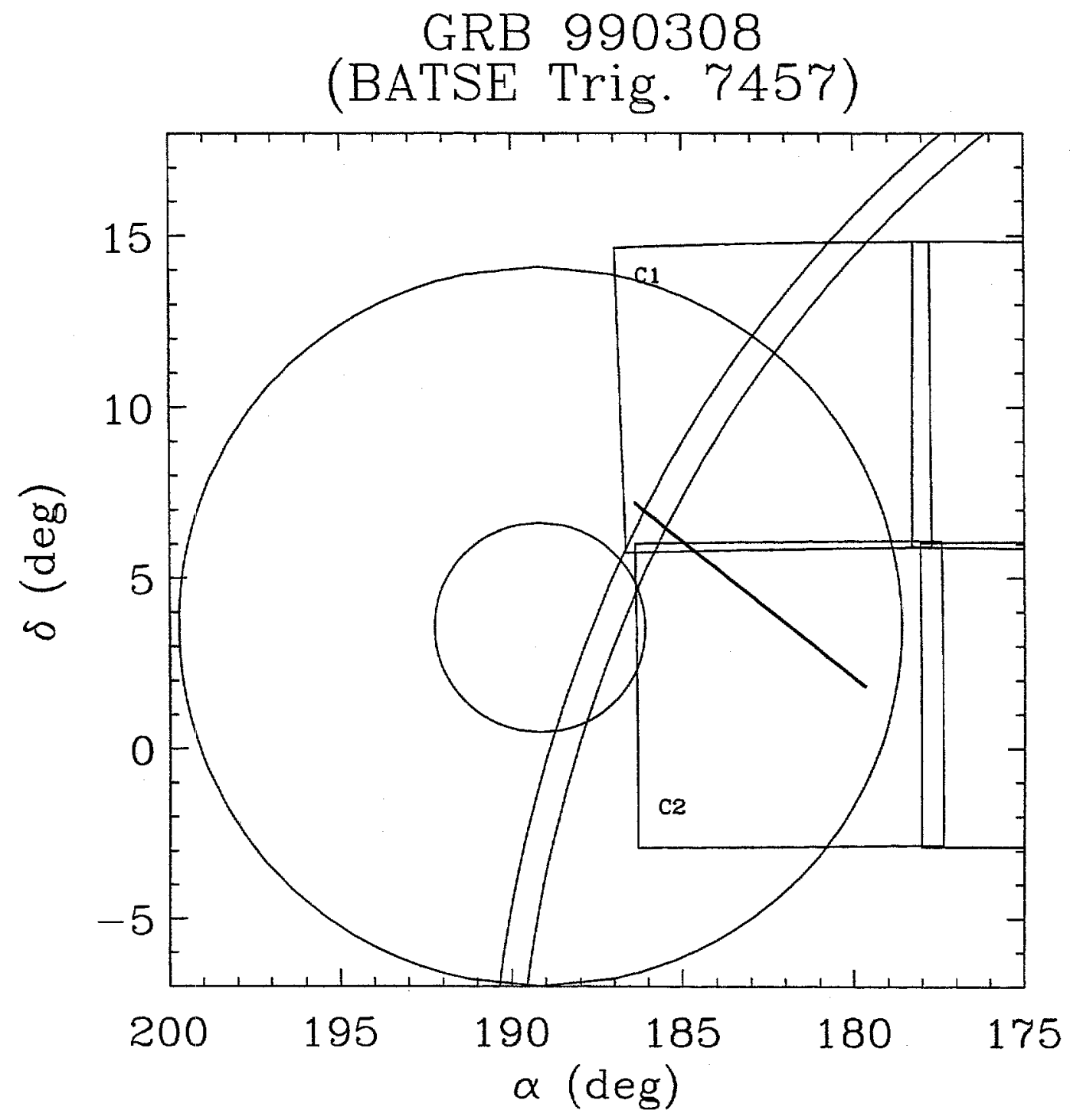

Figure 4.27 LOTIS coverage of GRB 990308; sixth image. The pseudo-squares represent the area covered by the four LOTIS cameras. The large circles represent the BATSE $1 \sigma$ and $3 \sigma$ error circles. The narrow arc is the IPN annulus. The long narrow box is the RXTE/ASM error box 


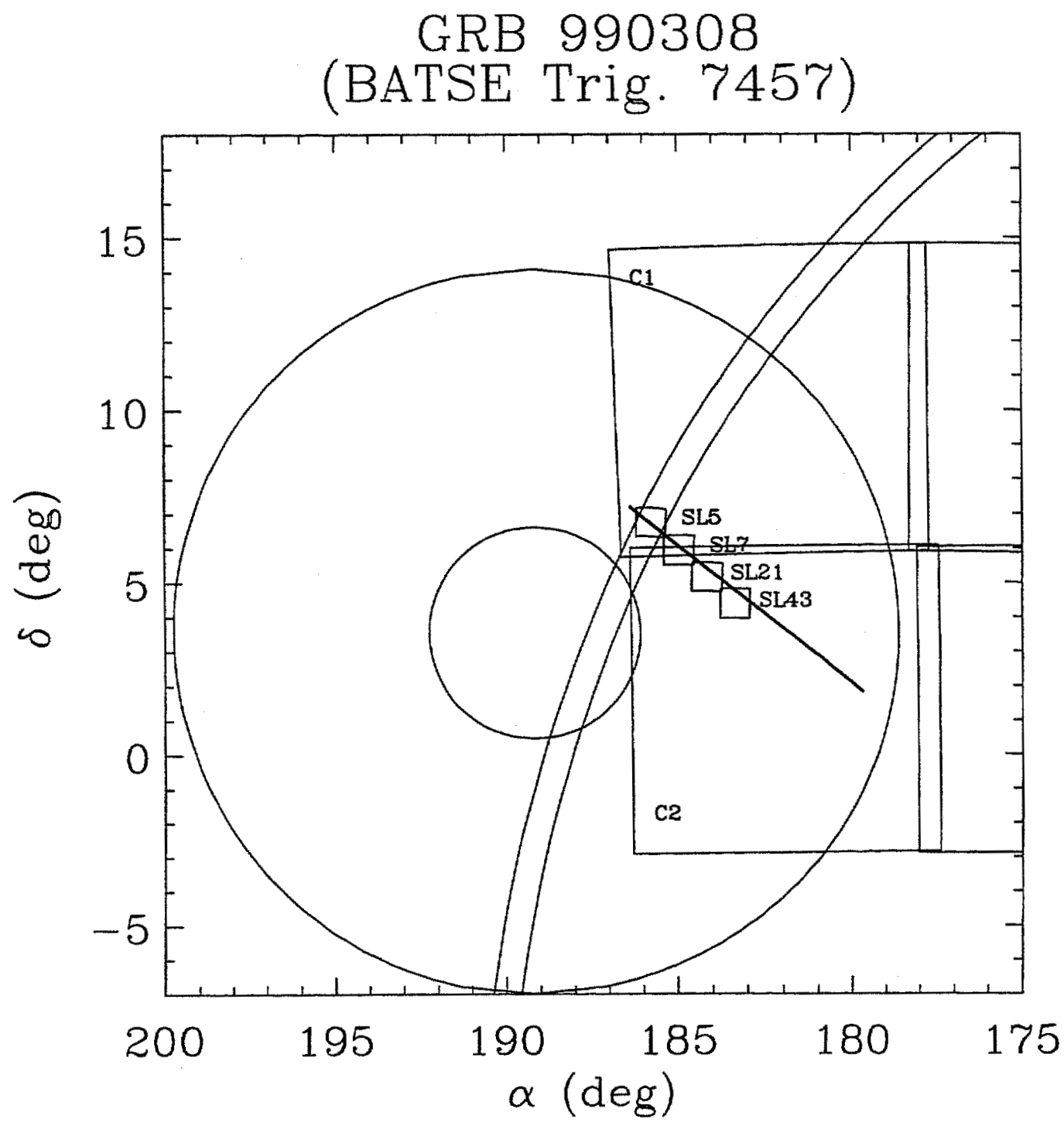

Figure 4.28 Super-LOTIS coverage of GRB 990308; wide field. The large pseudosquares represent the area covered by the four LOTIS cameras. The smaller pseudosquares represent the area along the RXTE error box covered by the Super-LOTIS raster scan. The large circles represent the BATSE $1 \sigma$ and $3 \sigma$ error circles. The narrow arc is the IPN annulus. 


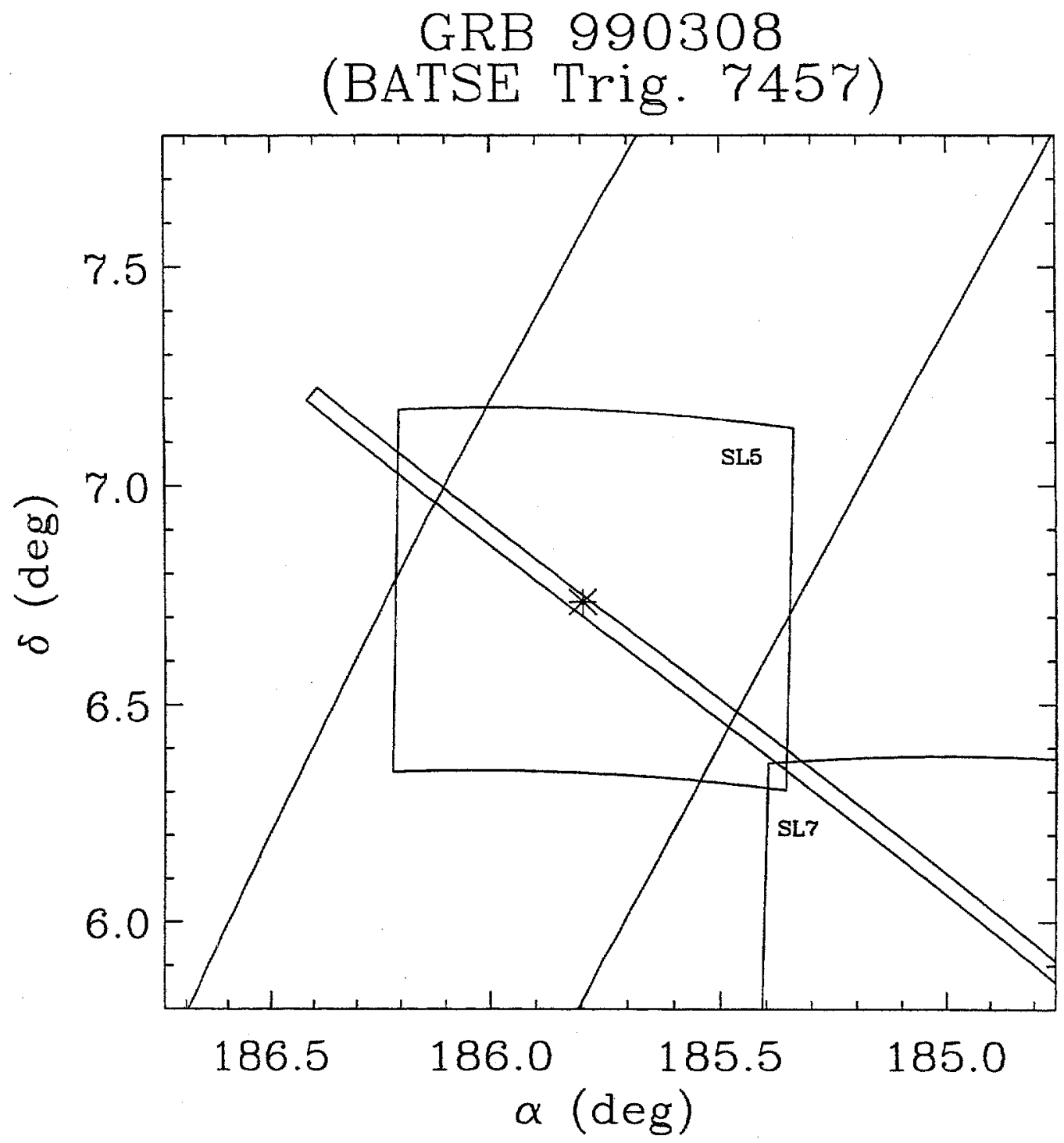

Figure 4.29 Super-LOTIS coverage of GRB 990308; narrow field. The pseudo-squares represent area along the RXTE error box, show as a narrow rectangle, covered by the Super-LOTIS raster scan. The wide arc is the IPN annulus. The star at the center shows the position of the optical counterpart discovered by Schaefer et al. (1999) 


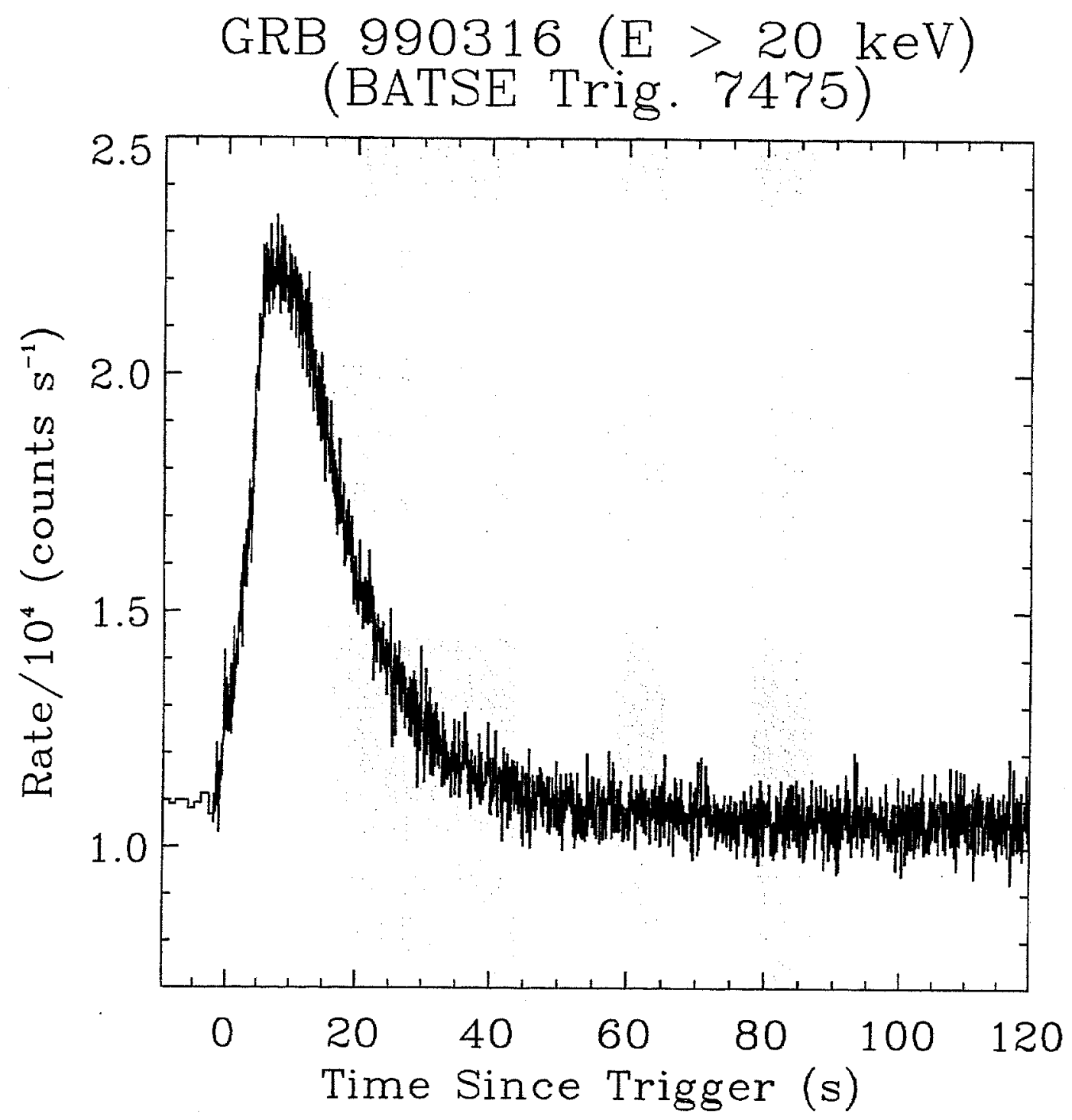

Figure 4.30 The BATSE gamma-ray light curve of GRB 990316. The grey shaded areas represent the integration times $(30 \mathrm{~s}, 10 \mathrm{~s}$, and $10 \mathrm{~s})$ of the first three LOTIS images. 


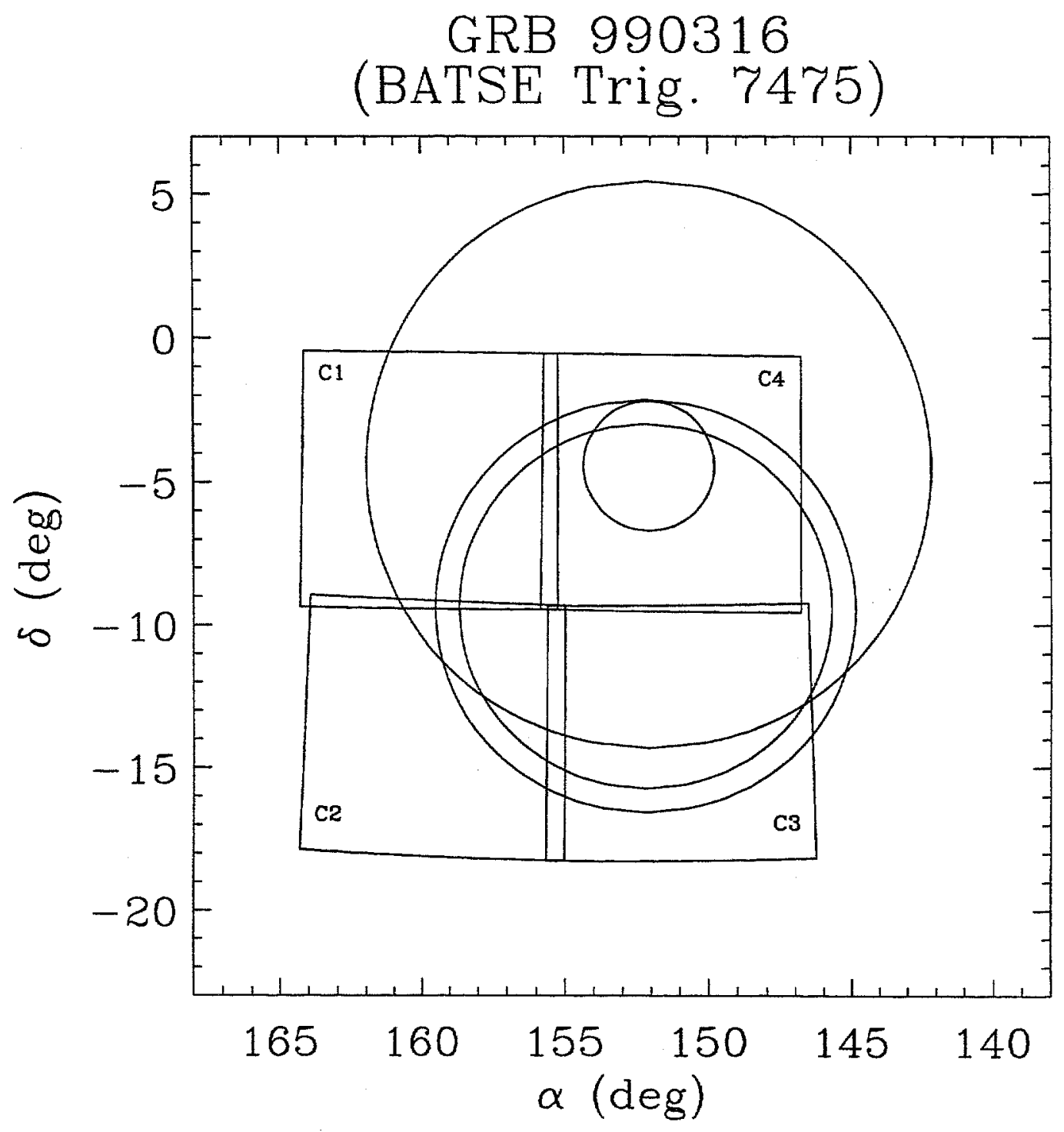

Figure 4.31 LOTIS coverage of GRB 990316. The pseudo-squares represent the area covered by the four LOTIS cameras. The large circles represent the BATSE $1 \sigma$ and $3 \sigma$ error circles. The narrow arc is the IPN annulus. 
GRB $990413(\mathrm{E}>20 \mathrm{keV})$ (BATSE Trig. 7518)

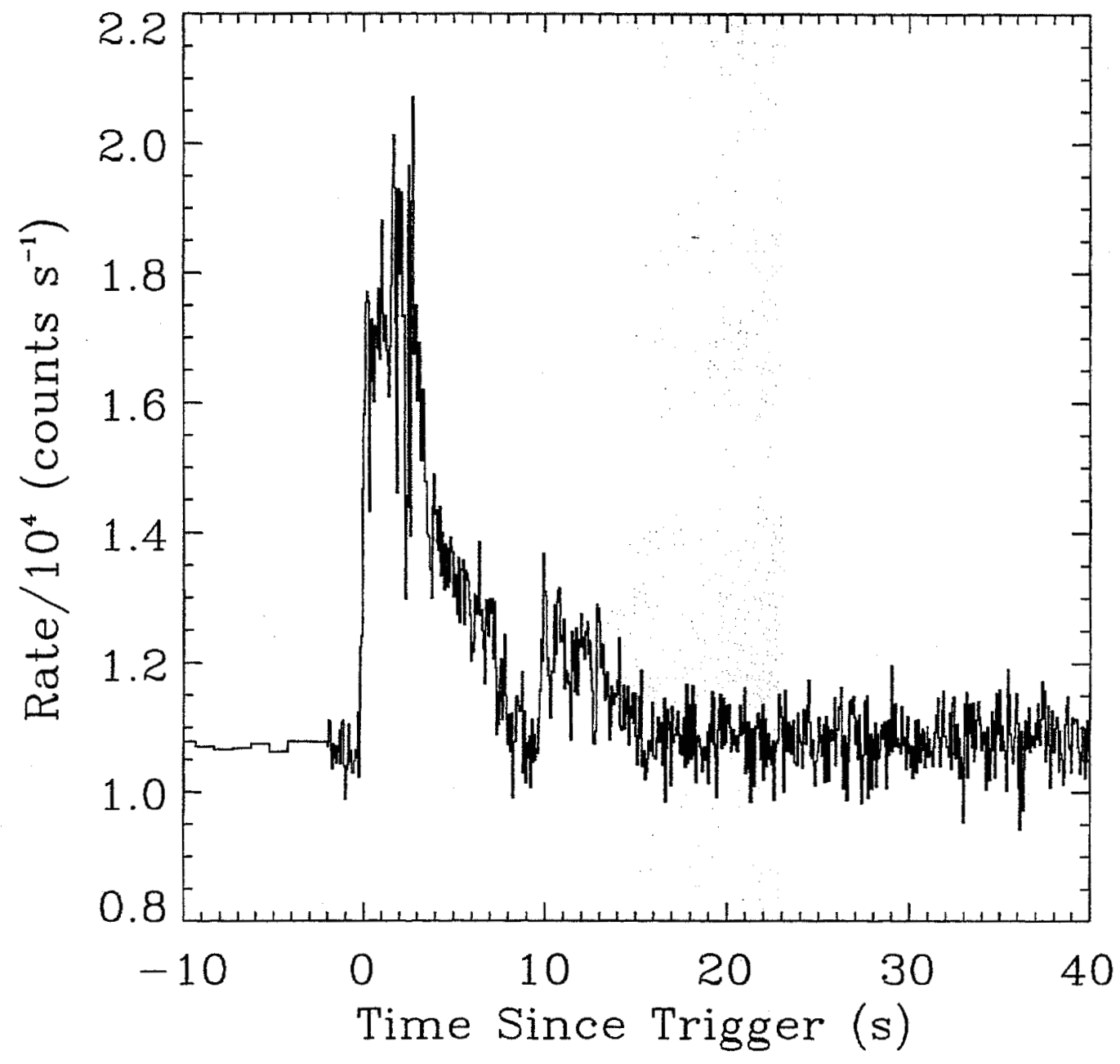

Figure 4.32 The BATSE gamma-ray light curve of GRB 990413. The grey shaded area represents the integration time of the first LOTIS image. 


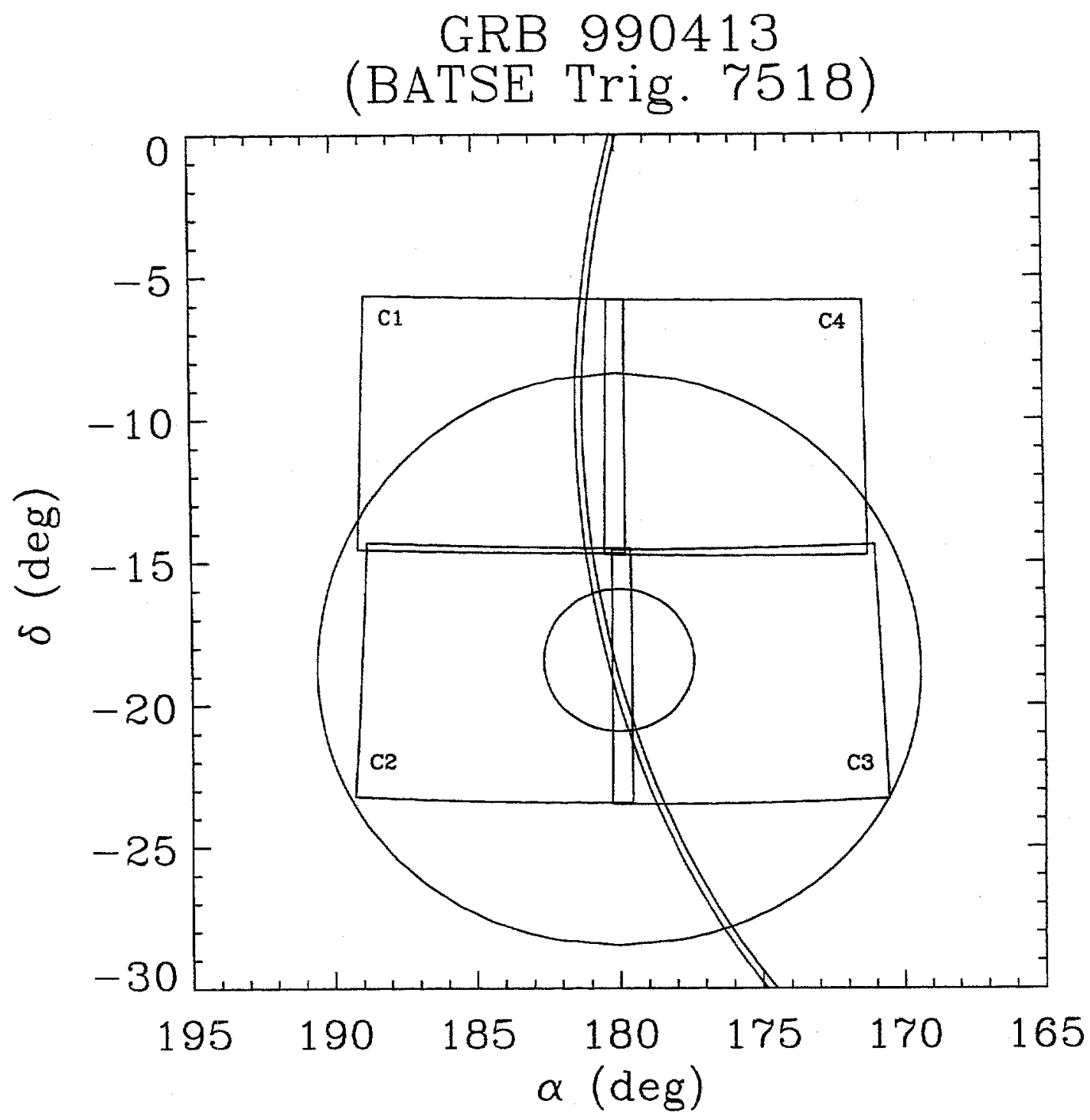

Figure 4.33 LOTIS coverage of GRB 990413. The pseudo-squares represent the area covered by the four LOTIS cameras. The large circles represent the BATSE $1 \sigma$ and $3 \sigma$ error circles. The narrow arc is the IPN annulus. 


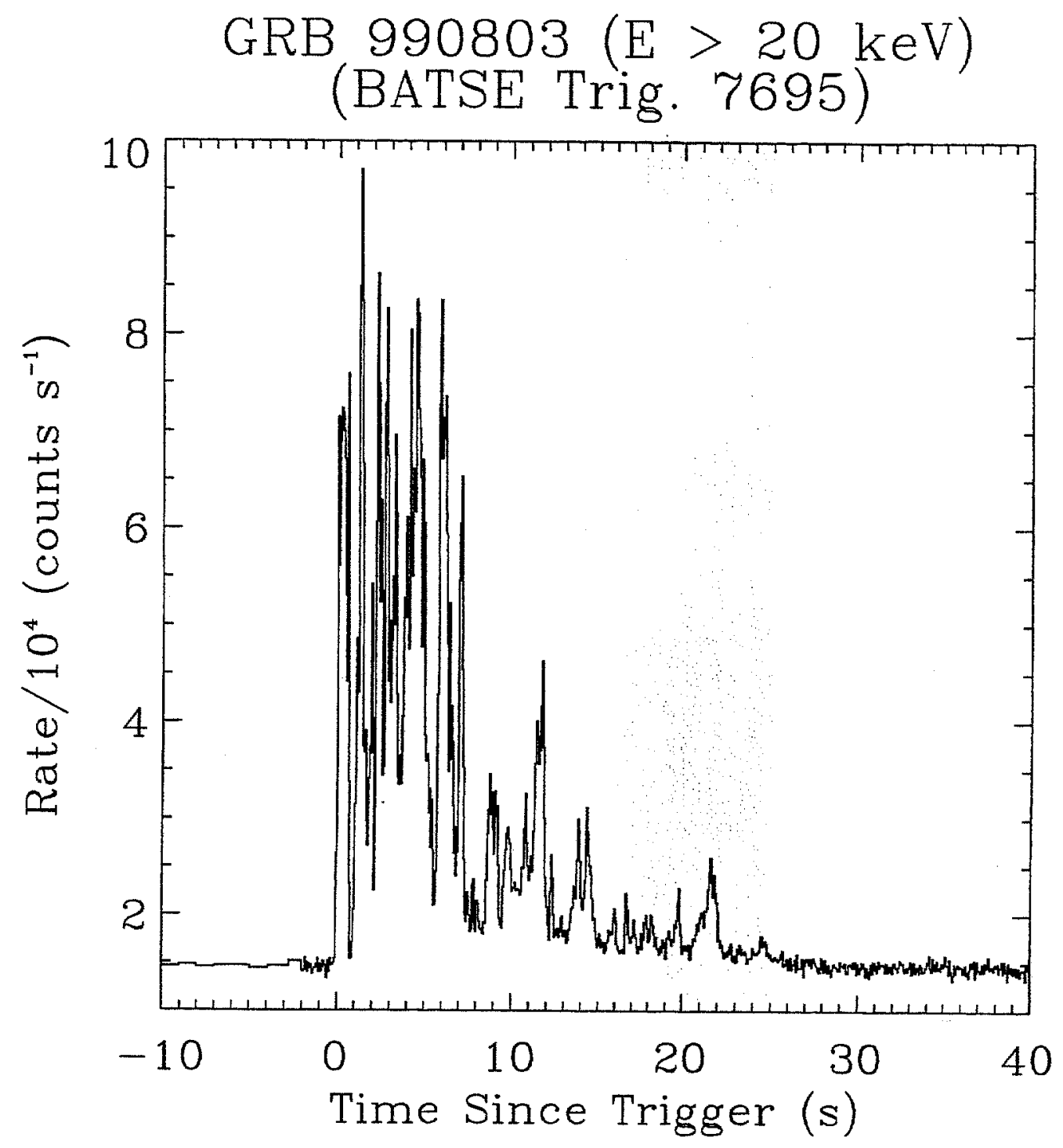

Figure 4.34 The BATSE gamma-ray light curve of GRB 990803. The grey shaded area represents the integration time of the first LOTIS image. 


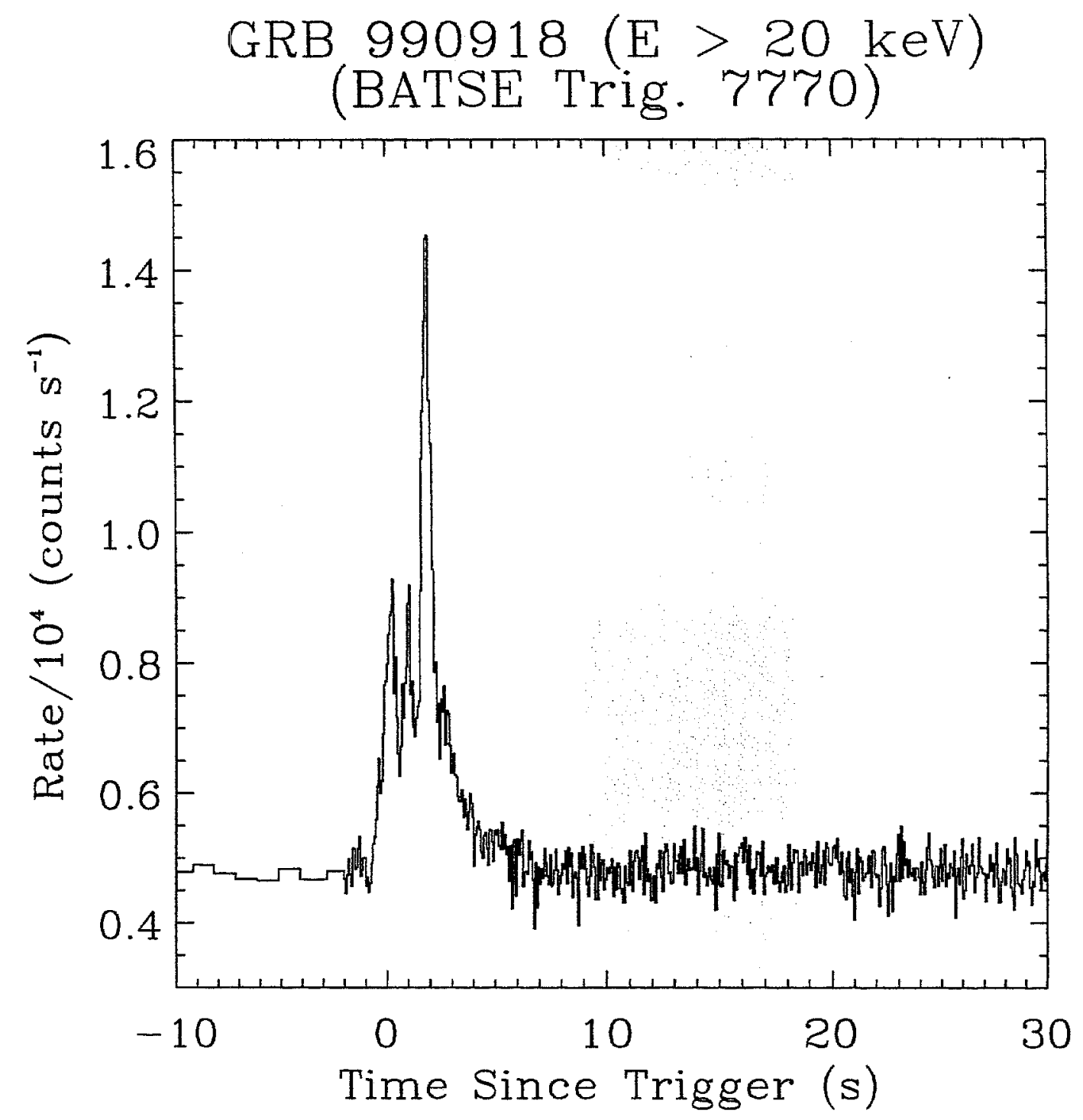

Figure 4.35 The BATSE gamma-ray light curve of GRB 990918. The grey shaded area represents the integration time of the first LOTIS image. 


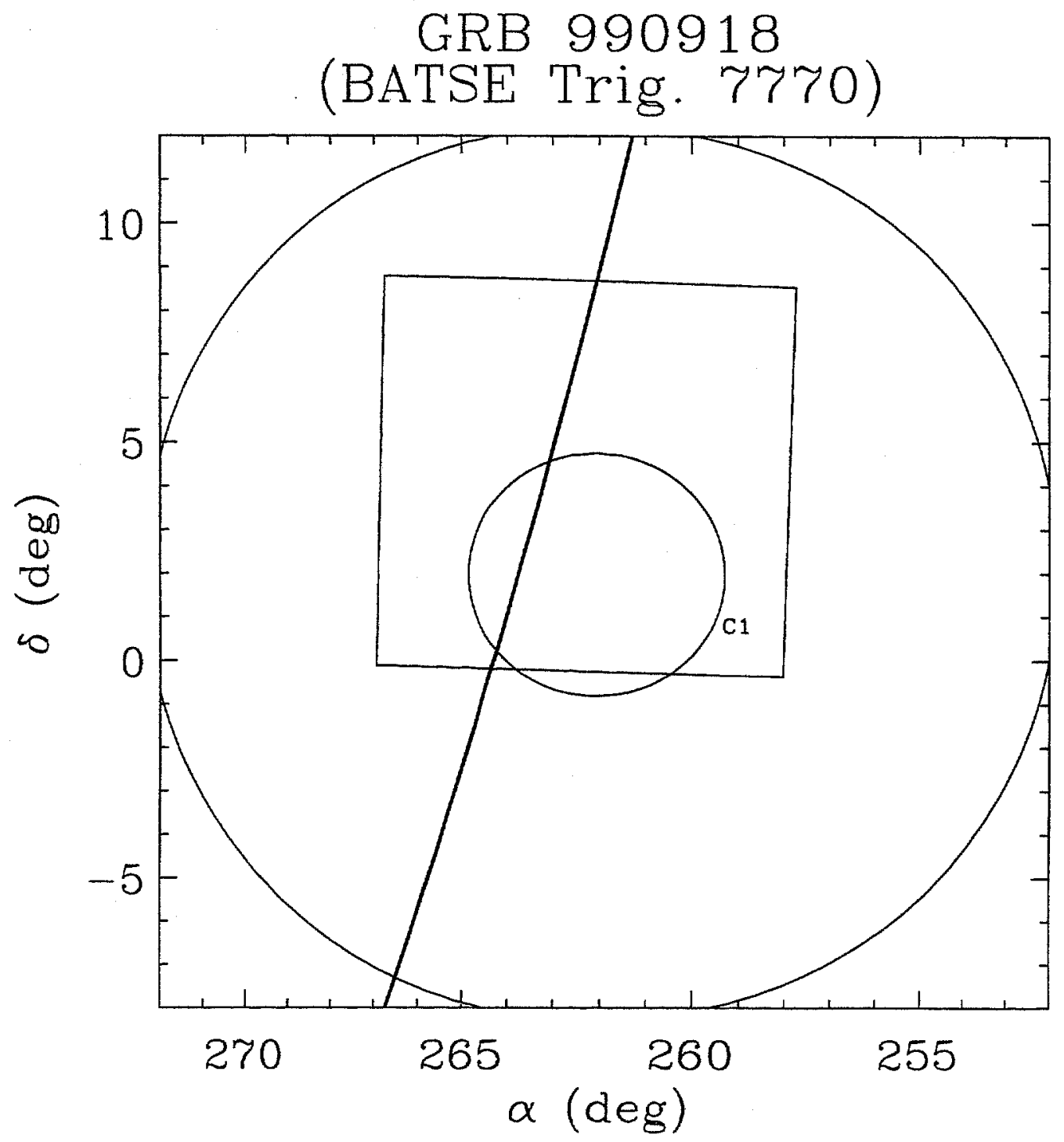

Figure 4.36 LOTIS coverage of GRB 990918. The pseudo-square represents the area covered by the LOTIS camera \#1. The large circles represent the BATSE $1 \sigma$ and $3 \sigma$ error circles. The narrow arc is the IPN annulus. 


\section{CHAPTER 5}

\section{DATA ANALYSIS AND INTERPRETATION}

\section{Data Reduction}

Basic data reduction for both LOTIS and Super-LOTIS consists of dark subtraction and flat fielding. Bias subtraction is not performed since the custom LLNL camera was not designed to obtain zero time exposures. The dark images contain the bias and therefore dark subtraction includes bias subtraction.

During a typical night at least two dark images per sky patrol are obtained for each planned integration time. When the sky patrol integration time is set to $30 \mathrm{~s}$ (10 s) the system obtains two (four) complete sky patrols. Two images are taken at each position in the sky patrol which results in four (eight) images of nearly every observable position. During a GRB trigger LOTIS obtains 20 minutes worth of data ( $~ 60$ images) before taking 5 dark images.

The LOTIS system suffers from considerable vignetting due to its wide fieldof-view. Therefore obtaining quality flats is important. However this is difficult to accomplish with an automated system which has fast optics. Twilight flats are generally not acceptable because of the ever-present sky gradient within the large fieldof-view. Median filter flats of up to 25 normal LOTIS images produce acceptable flats but often suffer from low level ghost stars throughout. The most successful LOTIS flats have been obtained during foggy nights. The scattered light from the water vapor produces images which are uniformly bright over the entire field. Super-LOTIS flats will be obtained through both twilight images and median filter sky patrol data.

Several techniques are employed to find optical transients (OTs) in the LOTIS GRB images. When LOTIS responds to a GRB trigger the system automatically sends e-mail to several pagers. This alert method was incorporated to facilitate rapid data analysis to increase the probability of early time follow-ups in the event that a transient is detected. 
The first step of LOTIS data reduction is typically subtraction of the first GRB image from a later image. Image number 30 is commonly used for the subtraction. The LOTIS integration time for GRB images is $10 \mathrm{~s}$ and the CCD readout and data storage take roughly $10 \mathrm{~s}$. The total time per image is approximately $20 \mathrm{~s}$ and so the time difference between the start of image number 1 and the start of image number 30 is $\sim 580 \mathrm{~s}$. Although LOTIS tracking keeps stars within a pixel during a $10 \mathrm{~s}$ integration, errors in the absolute polar alignment cause the stars to drift slightly from image to image. This drift is approximately 0.005 pixels per second. The drift from image number 1 to image number 30 is approximately 3 (2) pixels. Subtraction of the two images results in a stereo image of the stars. Any object which appears in only one of the two images clearly stands out.

Subtraction between several combinations of images is performed for each of the four cameras. The resultant images are inspected and all OTs are flagged. All flagged objects are inspected by eye to gauge the quality of their point-spread-function (psf). Many objects are tagged as obvious cosmic-ray hits because of an elongated or irregular psf. Single hot pixels and meteor trails are also disregarded. Objects which have psfs which are consistent with stars remain candidates for the optical counterpart.

All images which bracket an OT image are inspected for additional appearances. If the OT is not present in any other image, an average psf is calculated and compared with the psf of the OT. To date no transient object has appeared in more than one LOTIS image. If an OT psf fits that of the stars, more precise localization of the GRB is necessary to estimate the probability that a OT is associated with the GRB. More precise localization usually takes the form of the final Huntsville coordinates and an IPN arc. The transient object must appear within the final arc to be considered a candidate. To date only one transient, during GRB 990316, appeared on the edge but outside the IPN arc.

Following the preliminary subtraction method of locating OTs a more detailed study of the data is performed. Each of the approximately 60 GRB images is fully reduced. A computer algorithm is used to find brightness perturbations in all the 
images. Both custom algorithms and tested packages such as the IDL astronomy and IRAF "find" routines have been used. Each object is "followed", i.e. correlated with objects in subsequent images. The instrumental magnitude of each object is calculated and light curves are produced. All objects which display variability during the 60 images are flagged as counterpart candidates.

Several (5) stars in the first LOTIS GRB image are matched to known stars in the Guide Star Catalog (Jenkner et al. 1990) or the USNO-A1.0 Catalog (Monet 1996). The pixel positions and celestial coordinates of those stars are used to calculate a rotation matrix. The resultant rotation matrix is used to convert pixel coordinates to celestial coordinates for all detected objects. The coordinates of all the objects are compared to the coordinates of cataloged stars. Any object which appears in a LOTIS image but is not in the catalog is flagged as a counterpart candidate.

Finally the LOTIS images near the flagged objects are compared with the Digital Sky Survey (DSS). If any object remains a candidate, catalogs of known asteroids are checked.

Typically several objects are flagged which meet the above criteria but are not associated with the GRB. Objects which might be flagged include steady stars which are altered by light cloud cover, variable stars, dim stars at the LOTIS detection threshold, asteroids, and uncataloged stars.

\section{Interpretation}

Although considerable data is now available, LOTIS has not definitively detected prompt optical emission from a GRB. Interpretations of this null result include:

1. GRBs typically have bright prompt optical counterparts but LOTIS has failed to observe the location in most cases.

2. GRBs typically have bright prompt optical counterparts but extinction in the host galaxy renders them undetectable with LOTIS.

3. GRBs typically have bright prompt optical counterparts but a selection effect is constraining LOTIS observations to the dim end of the tail. 
4. GRBs typically have bright prompt optical emission but the duration is extremely short.

5. GRBs typically do not have bright prompt optical counterparts.

The results presented in Chapter 4 show that LOTIS achieved $100 \%$ probability of covering the positions of GRB 971006, GRB 971227, and GRB 990308. In addition LOTIS achieved greater than $95 \%$ probability of covering the positions of GRB 961017, GRB 961220, GRB 970223, GRB 990316, and GRB 990413. Therefore we argue against the first explanation above.

There is evidence from GRB afterglow data that at least some GRB host galaxies have less than two magnitudes of extinction along the line of sight (e.g., Reichart 1998; Vreeswijk et al. 1999). Even if extinction reduced the prompt optical emission by two magnitudes bright events such as GRB 990123 could be detected by LOTIS. In the case of GRB 990308 the optical counterpart was detected at $\mathrm{R}=$ $18.14 \pm 0.06,200$ minutes after the burst. This is on the bright end of the distribution of magnitudes for optical afterglows. Therefore the extinction in this case is not likely to be much greater than for other bursts. It is possible that the extinction varies with time due to the GRB itself however we argue against the second explanation above.

The characteristics of the GRBs which LOTIS has observed span the the entire parameter space. LOTIS has obtained upper limits for both short $(\sim 1 \mathrm{~s})$ and long (> $100 \mathrm{~s}$ ) bursts, smooth bursts and extremely variable bursts, bursts with large peak flux and bursts with low peak flux, bursts with high fluence and bursts with low fluence. There may be combinations of characteristics which result in bright prompt optical emission however the variety of events that LOTIS has obtained upper limits for argues against selection effects.

LOTIS has upper limits for several bursts within 10-20 s of the start of the burst. In many of these cases the LOTIS observations were simultaneous with the gamma-ray emission. If prompt optical emission from GRBs is too short to be observed, the emission must commonly be shorter than the the gamma-ray emission. This is unlikely if bursts are the result of relativistic blast waves (Sari \& Piran 1999b). 
In the case of GRB 990123 the peak of the prompt optical emission followed the peak of the gamma-ray emission. Although the prompt emission faded rapidly it was still above the LOTIS detection threshold more than $500 \mathrm{~s}$ after the burst (Akerlof et al. 1999). We argue against short bright prompt optical emission.

We have argued against the first four interpretations given above. There may be many more interpretations of the LOTIS null results however we suggest that GRBs are not typically accompanied by bright prompt optical emission.

The LOTIS data suggests that GRBs are not typically accompanied by bright prompt optical emission. With this assumption and a model for the emission mechanism the physical parameter space can be constrained by the LOTIS results. In the following interpretation the prompt optical emission is assumed to originate in the external reverse shock. Other models will be constrained in different ways.

GRB 990123 is the only event for which prompt optical emission was detected. Comparison of LOTIS results with GRB 990123 is necessary however it should be noted that GRB 990123 was not a typical GRB. Its characteristics, including peak flux, fluence, and spectral hardness, were all in the extreme tails of their respective distributions (Briggs et al. 1999a). Any, and perhaps all, of these extreme characteristics led to the bright prompt optical emission. Regardless, GRB 990123 is the only available comparison and is therefore used as such. In these cases we attempted to allow for proper scaling.

\section{Scaled Burst Properties}

First the LOTIS upper limits are compared with GRB 990123 to test whether the flux of the prompt optical emission scales with some gamma-ray property. Here and throughout the analysis we neglect extinction effects. The first row in Table IV. lists the properties of GRB 990123 (Briggs et al. 1999a; Akerlof et al. 1999). The columns display the UTC date of the burst, the BATSE trigger number, the $64 \mathrm{~ms}$ and 1024 ms peak flux $(50-300 \mathrm{keV})$, and the gamma-ray fluence $(>20 \mathrm{kev})$ of each event. The last three columns are the scaled optical magnitudes calculated using,

$$
m_{\mathrm{GRB}}=m_{\mathrm{GRB} 990123}-2.5 \log \left(\frac{X_{\mathrm{GRB}}}{X_{\mathrm{GRB} 990123}}\right) \text {, }
$$


where $m_{\mathrm{GRB} 90123}=9.0$ is the peak magnitude of GRB 990123 , and $X_{\mathrm{GRB}}$ and $X_{\mathrm{GRB} 990123}$ are the peak Alux or fluence values for those events.

Table IV. LOTIS GRB events scaled to GRB 990123. Predictions for the magnitudes of the prompt optical emission.

\begin{tabular}{cccccccc} 
Date & $\begin{array}{c}\text { BATSE } \\
\text { Trig. }\end{array}$ & $\begin{array}{c}F_{p}(64 \mathrm{~ms}) \\
\left(\gamma \mathrm{cm}^{-2} \mathrm{~s}^{-1}\right)\end{array}$ & $\begin{array}{c}F_{p}(1024 \mathrm{~ms}) \\
\left(\mathrm{erg} \mathrm{cm}^{-2}\right)\end{array}$ & $\begin{array}{c}S / 10^{-7} \\
m_{F ; 64}\end{array}$ & $m_{F ; 1024}$ & $m_{S}$ \\
\hline 990123 & 7343 & 16.96 & 16.41 & 3000. & 9.0 & 9.0 & 9.0 \\
\hline 961017 & 5634 & 4.22 & 1.98 & 5.07 & 10.5 & 11.3 & 15.9 \\
961220 & 5719 & 1.93 & 1.60 & 18.11 & 11.4 & 11.5 & 14.5 \\
970223 & 6100 & 19.41 & 16.84 & 968. & 8.9 & 9.0 & 10.2 \\
970714 & 6307 & 1.89 & 1.32 & 17.09 & 11.4 & 11.7 & 14.6 \\
970919 & 6388 & 1.10 & 0.77 & 22.49 & 12.0 & 12.3 & 14.3 \\
971006 & 6414 & 2.08 & 1.79 & 258. & 11.3 & 11.4 & 11.7 \\
971227 & 6546 & 3.32 & 2.11 & 9.25 & 10.8 & 11.2 & 15.3 \\
990129 & 7360 & 5.88 & 4.99 & 585. & 10.2 & 10.3 & 10.8 \\
990308 & 7457 & 2.02 & 1.26 & 164. & 11.3 & 11.8 & 12.2 \\
990316 & 7475 & 3.87 & 3.67 & 529. & 10.6 & 10.6 & 10.9 \\
990413 & 7518 & 3.78 & 2.57 & 68.13 & 10.6 & 11.0 & 13.1 \\
990803 & 7695 & 16.99 & 12.19 & 1230. & 9.0 & 9.3 & 10.0 \\
990918 & 7770 & 5.69 & 3.17 & 25.21 & 10.2 & 10.8 & 14.2 \\
\hline
\end{tabular}

The LOTIS upper limits for each event are given in Table II. The system sensitivity varies with observing conditions but a conservative limiting magnitude is $m \approx 11.5$ prior to March 1998 (upgrade to cooled CCD) and $m \approx 14.0$ following that date. Table IV. shows that the scaled prompt optical emission for both peak flux and fluence is often brighter than the LOTIS upper limits. This suggests that these 
simple relationships are not valid. Akerlof et al. (2000) also find that the scalings are not appropriate.

Spectral Extrapolation

Briggs et al. (1999a) showed that the optical flux measured during GRB 990123 is not consistent with an extrapolation of the burst spectrum to low energies. However Liang et al. (1999) pointed out that the extrapolated tails rise and fall with the optical flux. A low energy enhancement might produce an upward break which could account for the measured optical flux during GRB 990123. If this type of upturn exists it may suggest that the optical and gamma-ray photons are produced by the same electron distribution. The LOTIS upper limits can be used to constrain a low energy enhancement assuming it is common to all GRBs.

For four events listed in Table IV. we fit the gamma-ray spectra during the LOTIS observations to the Band functional form (Band et al. 1993). The solid lines in Figure 5.1 show these fits and the extrapolation to low energies. Fits to the spectra of GRB 990123 during the first (short dash), second (dash-dot), and third (long dash) ROTSE observations and the corresponding ROTSE detections (filled circles) are also shown.

For two cases shown in Figure 5.1 the low energy extrapolation alone is near the LOTIS upper limit. More specifically, the extrapolation of GRB 971006, predicts an $m \approx 12.4$ optical flash. Even a slight upward break in the spectrum would have produced a detectable OT. The LOTIS upper limits support the hypothesis that the low energy emission is produced by a different electron distribution than the high energy emission.

\section{Reverse Shock Constraints}

Finally LOTIS upper limits and the external reverse shock model are used to constrain the physical properties of the GRB blast wave. In this analysis a uniform CBM density is assumed. Sari \& Piran (1999b) show that the fraction of the burst energy which gets emitted in the optical band depends on the values of the cooling 
frequency and the characteristic synchrotron frequency. For the external reverse shock these frequencies are given by,

$$
\nu_{c}=8.8 \times 10^{15} \mathrm{~Hz}\left(\frac{\epsilon_{B}}{0.1}\right)^{-3 / 2} E_{52}{ }^{-1 / 2} n_{1}{ }^{-1} t_{A}^{-1 / 2},
$$

and

$$
\nu_{m}=1.2 \times 10^{14} \mathrm{~Hz}\left(\frac{\epsilon_{e}}{0.1}\right)^{2}\left(\frac{\epsilon_{B}}{0.1}\right)^{1 / 2}\left(\frac{\gamma_{0}}{300}\right)^{2} n_{1}^{1 / 2},
$$

where $\epsilon_{e}$ and $\epsilon_{B}$ are the fraction of equipartition energy in the electrons and magnetic field, $E_{52}$ is the total energy in units of $10^{52} \mathrm{erg}, n_{1}$ is the density of the CBM in $\mathrm{cm}^{-3}$, $\gamma_{0}$ is the initial Lorentz factor, and $t_{A}$ is the duration of the emission in seconds.

The flux of prompt optical emission or the peak optical magnitude of a GRB can be estimated from five blast wave parameters and two observable parameters. The blast wave parameters are $E_{52}, \epsilon_{B}, \epsilon_{e}, \gamma_{0}$, and $n_{1}$. The observable parameters are $S$ the gamma-ray fluence and $t_{A}$ the approximate length of the burst (or the integration time if it is less than the length of the emission). These parameters may depend on one another but the relationships are not well understood and are assumed independent.

If the energy of a burst with a slightly larger than average fluence of $S=$ $10^{-5} \mathrm{erg} \mathrm{cm}^{-2}$ were peaked in the R-band and had a duration of approximately $10 \mathrm{~s}$ one would expect an optical flash to occur with a flux of

$$
f_{\nu} \approx 1 / 4 \times \frac{10^{-5} \mathrm{erg} \mathrm{cm}^{-2}}{10 \mathrm{~s} \times 5 \times 10^{14} \mathrm{~Hz}}=50 \mathrm{Jy} \Rightarrow m_{R} \sim 5 .
$$

The leading factor of $1 / 4$ is an approximation for the amount of emission above the peak frequency, which typically has an $F_{\nu} \sim \nu^{-1.25}$ dependence (Sari \& Piran 1999b).

Equation 5.4 provides an estimate of the maximum possible peak optical flux for a GRB which has a fluence of $S=10^{-5} \mathrm{erg} \mathrm{cm}^{-2}$. That is, $m_{R} \geq 5$ always. The peak flux will be altered by the fraction of energy that goes into the reverse shock and a factor which depends on the relative positions of the break frequencies and the observation band. 
Table V. The fraction of the energy that is emitted at the optical frequency, $\nu_{o p}$, as function of the cooling frequency, $\nu_{c}$, and the characteristic synchrotron frequency, $\nu_{m}$.

\begin{tabular}{|c||c|c|}
\hline & $\nu_{c}>\nu_{o b}$ & $\nu_{c}<\nu_{o b}$ \\
\hline \hline$\nu_{m}>\nu_{o b}$ & $\left(\frac{\min \left(\nu_{c}, \nu_{m}\right)}{\max \left(\nu_{c}, \nu_{m}\right)}\right)^{1 / 2}\left(\frac{\nu_{o b}}{\min \left(\nu_{c}, \nu_{m}\right)}\right)^{4 / 3}$ & $\left(\frac{\nu_{o b}}{\nu_{m}}\right)^{1 / 2}$ \\
\hline$\nu_{m}<\nu_{o b}$ & $\left(\frac{\nu_{o b}}{\nu_{m}}\right)^{-(p-2) / 2}\left(\frac{\nu_{o b}}{\nu_{c}}\right)^{1 / 2}$ & $\left(\frac{\nu_{o b}}{\nu_{m}}\right)^{-(p-2) / 2}$ \\
\hline
\end{tabular}

Following the discussion in Chapter 2 and the spectral shapes given in Figure 2.1 the flux in the optical band can be calculated from the values of the cooling break and characteristic synchrotron break. Table V. shows the fraction of the peak flux emitted in the optical band as a function of these break frequencies.

Different blast wave parameters can be used in Equations 5.2 and 5.3. to determine the cooling frequency, $\nu_{c}$, and the characteristic synchrotron frequency, $\nu_{m}$. Table V. can then be used to predict the flux of the prompt optical emission for those blast wave parameters.

Figure 5.2 shows a contour plot of the peak optical magnitude as a function of the density of the CBM, $n_{1}$, and the initial Lorentz factor, $\gamma_{0}$. The other blast wave parameters have been fixed at $E_{52}=5.0, \epsilon_{e}=\epsilon_{B}=0.5$ and the observable parameters are fixed at $S=2.33 \times 10^{-7} \mathrm{erg} \mathrm{cm}^{-2}$ and $t_{A}=10.0$. There are three discontinuities in the plot which indicate different relationships between the break frequencies and the observation frequency. The points $\mathrm{A}, \mathrm{B}, \mathrm{C}$, and D appear in regions bracketed by the discontinuities. The corresponding spectra for each of these points is shown in Figure 5.3. The grey shaded area represents the R-band. Spectra A, B, C, and D have the following spectral characteristics, $\nu_{m}<\nu_{o b}<\nu_{c}, \nu_{o b}<\nu_{m}<\nu_{c}, \nu_{o b}<\nu_{c}<\nu_{m}$, and $\nu_{c}<\nu_{o b}<\nu_{m}$, respectively.

These contour plots can be used to explore the predicted prompt optical emission for the entire blast wave parameter space. Figure 5.4 shows nine contour plots of the predicted prompt optical emission. Each of the plots is a function of the density of the CBM, $n_{1}$, and the initial Lorentz factor, $\gamma_{0}$. The values of the fraction of equipar- 
Figure 5.3. The grey shaded area represents the R-band. Spectra A, B, C, and D have the following spectral characteristics, $\nu_{m}<\nu_{o b}<\nu_{c}, \nu_{o b}<\nu_{m}<\nu_{c}, \nu_{o b}<\nu_{c}<\nu_{m}$, and $\nu_{c}<\nu_{o b}<\nu_{m}$, respectively.

These contour plots can be used to explore the predicted prompt optical emission for the entire blast wave parameter space. Figure 5.4 shows nine contour plots of the predicted prompt optical emission. Each of the plots is a function of the density of the CBM, $n_{1}$, and the initial Lorentz factor, $\gamma_{0}$. The values of the fraction of equipartition energy in the electrons, $\epsilon_{e}$, and magnetic field, $\epsilon_{B}$, increase from left-to-right and top-to-bottom. The burst energy has been fixed at $E_{52}=5.0$ consistent with afterglow measurements. The length of the emission is fixed at the LOTIS integration time of $t_{A}=10.0$. If the emission occurs over a shorter time-scale the base magnitude can be adjusted properly. The fluence is fixed at $S=2.33 \times 10^{-7} \mathrm{erg} \mathrm{cm}^{-2}$ less than the fluence of any LOTIS event given in Table IV.

The increased shading in the contour plots indicate regions of parameter space where LOTIS is less likely to detect the prompt optical emission. The light regions in the contour plots are excluded by the LOTIS upper limits. The values of the blast wave parameters which are most consistent with the LOTIS upper limits are low circumburster density, high initial Lorentz factors, low equipartition energy in the magnetic field, and high equipartition energy in the electrons.

If the prompt optical emission from GRBs is produced by synchrotron emission in the reverse external shock and the shape of the spectrum is well described by the break frequencies in Equations 5.2 and 5.3 then the contour plots given here provide a graphical description of the dependencies of the optical emission on the blast wave parameters. The values of the contour lines may change according to a number of factors but their shapes will remain the same and the LOTIS upper limits tend to exclude certain regions of parameter space. 

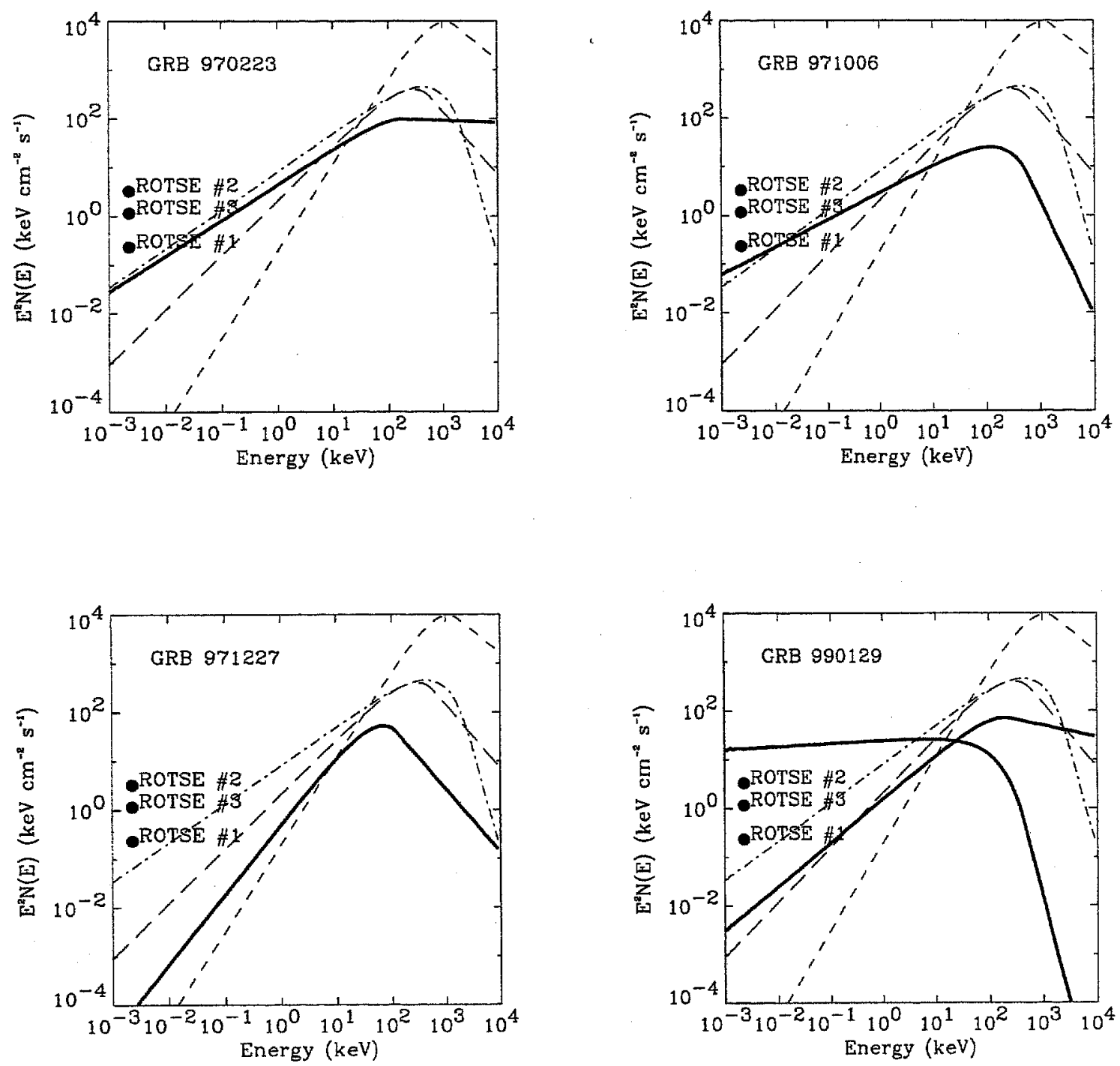

Figure 5.1 Extrapolated spectra of LOTIS GRBs. 


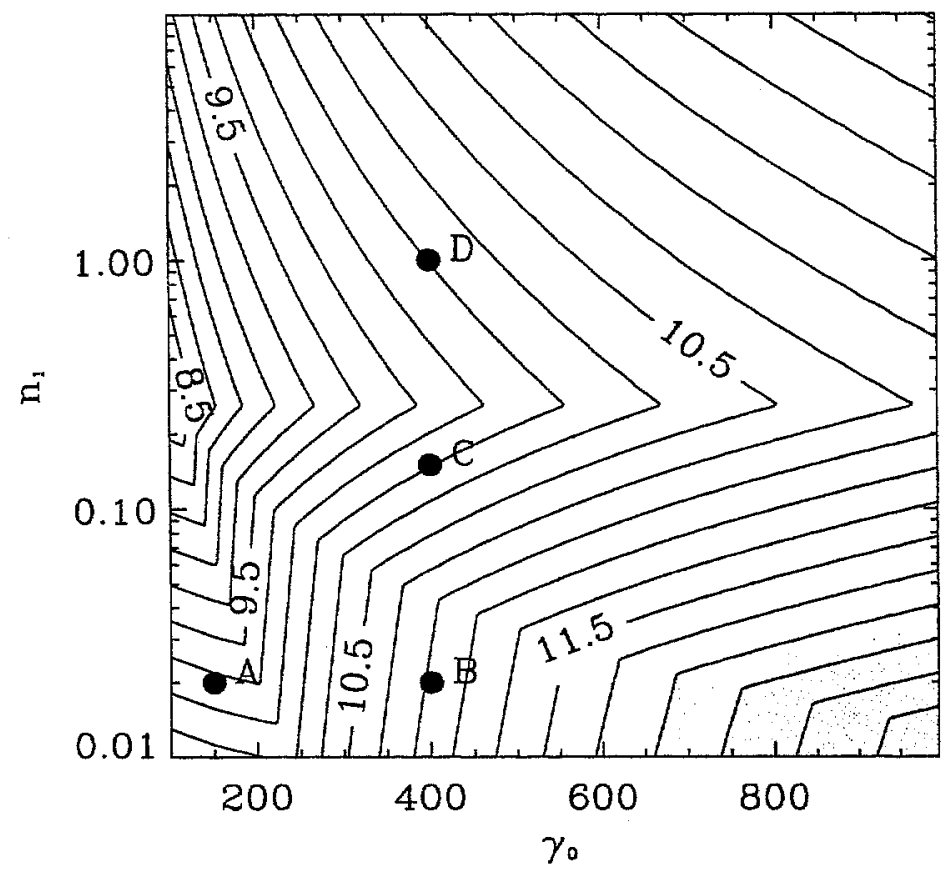

Figure 5.2 Magnitude of the prompt optical emission from the reverse shock. The points $\mathrm{A}, \mathrm{B}, \mathrm{C}$, and $\mathrm{D}$ correspond to parameters of the corresponding spectra. $E_{52}=$ $5.0, t_{A}=10.0, \epsilon_{e}=\epsilon_{B}=0.5, S=2.33 \times 10^{-7} \mathrm{ergcm}^{-2}$ 

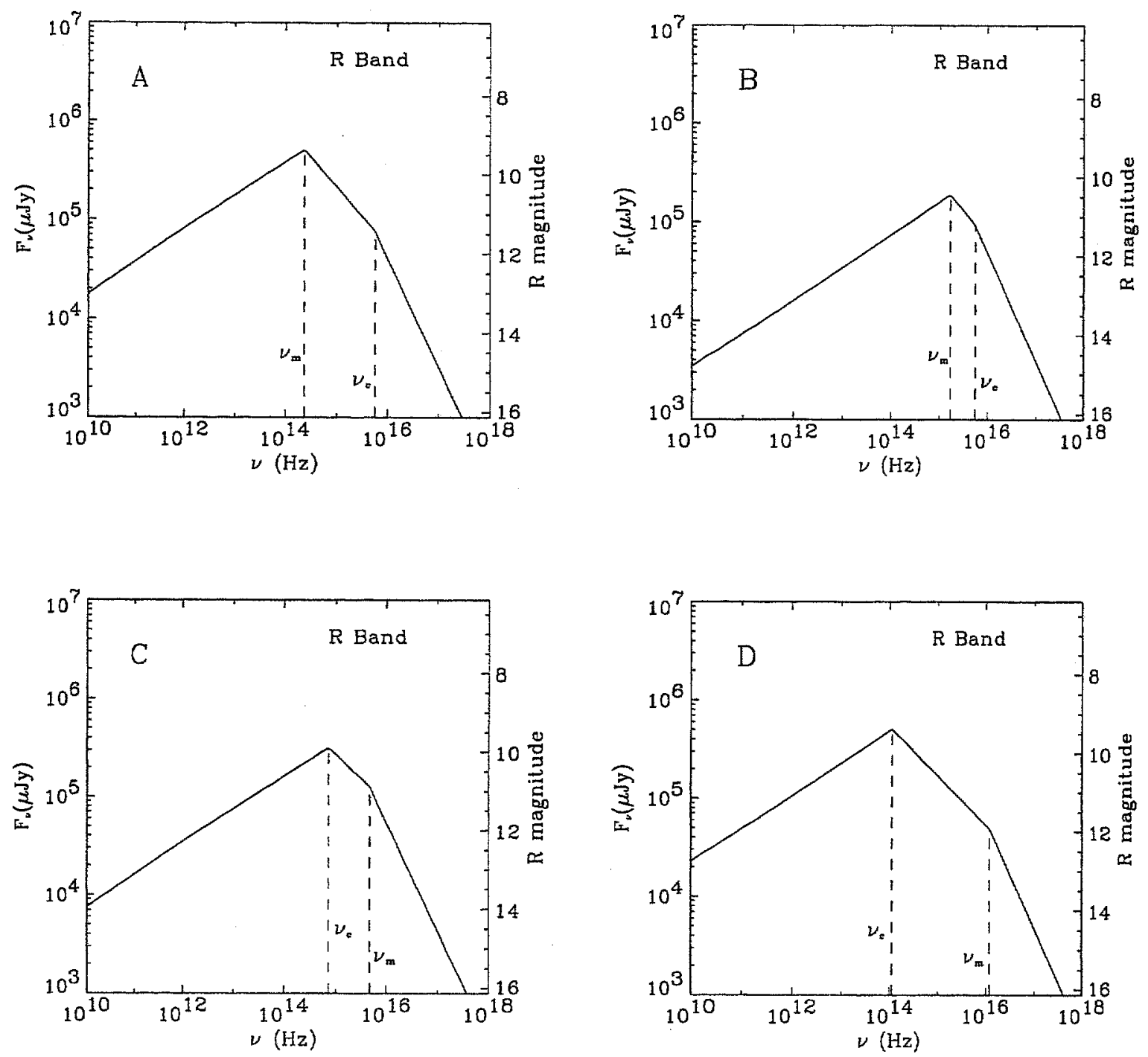

Figure 5.3 Prompt optical synchrotron spectra corresponding to points A, B, C, and $\mathrm{D}$ in the contour plot. 


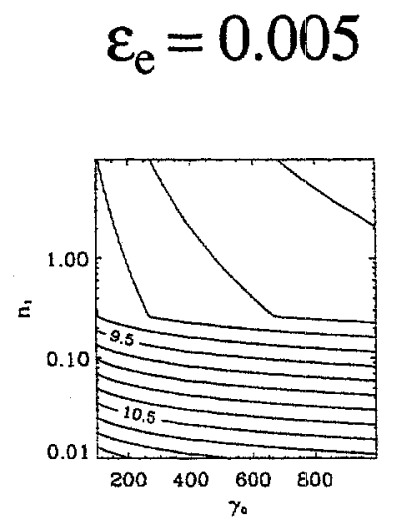

$$
\varepsilon_{\mathrm{e}}=0.05
$$

$$
\varepsilon_{\mathrm{e}}=0.5
$$
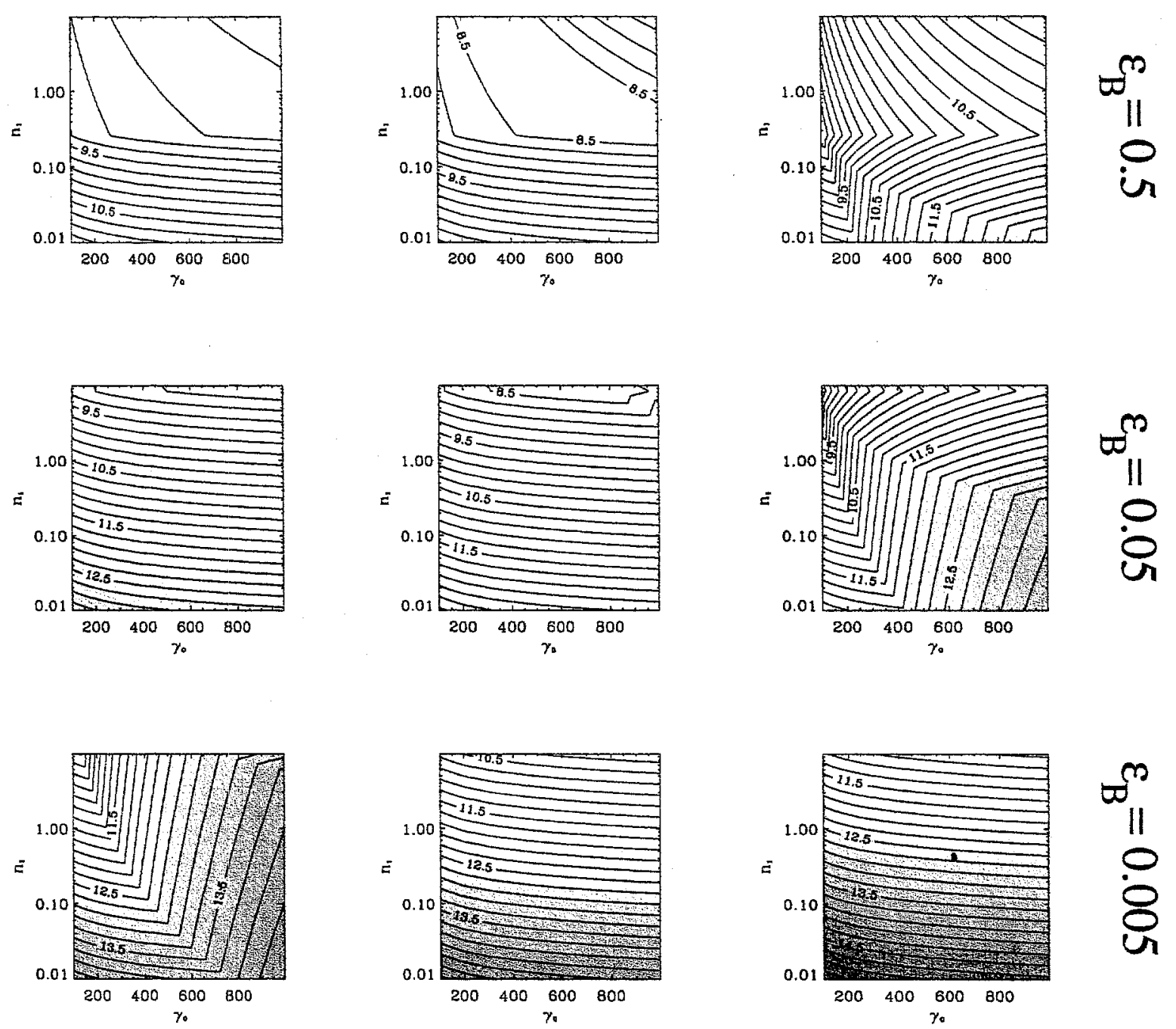

Figure 5.4 Prompt optical emission from the reverse shock using the range of likely parameter space. 


\section{CHAPTER 6 \\ CONCLUSIONS}

\section{Conclusions}

The objective of the LOTIS experiment is to detect or provide upper limits of the prompt optical emission from GRBs. The experiment has successfully accomplished this objective. Results from 13 LOTIS GRB observations have been presented and analyzed in this thesis. Figure 6.1 shows the LOTIS upper limits from these 13 events plotted together with other published upper limits (Hudec 1995; Vanderspek et al. 1992; Park et al. 1997a; Akerlof et al. 2000). The prompt optical emission from GRB 990123 is plotted as a star (Akerlof et al. 1999). The open circle represents upper limits or detections expected from Super-LOTIS in the near future. The data suggest that GRBs are not typically accompanied by bright prompt optical emission.

The LOTIS results also show that the level of the prompt optical emission does not scale with GRB peak flux or fluence. Extrapolations of the burst spectra to low energies is ruled out by GRB 990123 as well as the LOTIS upper limits.

Assuming the prompt optical emission is produced by synchrotron emission in the external reverse shock the LOTIS data have been used to constrain the blast wave parameter space. The LOTIS upper limits favor low circumburster density, high initial Lorentz factors, low equipartition energy in the magnetic field, and high equipartition energy in the electrons.

\section{Future Plans}

With the recent proliferation in GRB localizations and the planned HETE2 and Swift missions both LOTIS and Super-LOTIS are likely to make significant contributions to the GRB field in the future. The de-orbit of CGRO removed the only available source of real-time GRB localizations. However HETE-2 should localize an estimated 50 bursts per year in real-time to better than $10^{\prime}$. To accommodate the smaller error boxes the LOTIS cameras will be collapsed to provide a single field-of- 
view in all four cameras. The cameras will be equipped with various filters to provide early time simultaneous multiband photometry.

Super-LOTIS provides sensitivity necessary to definitively determine the typical level of the prompt optical emission. Early time observations can distinguish between dim afterglows caused by extinction and dim afterglows cause by rapid fading.

Both instruments will be used for ancillary science. LOTIS will produce a continuous catalog of the sky in at least three colors. This catalog will be used to identify and classify variable astrophysical sources. Super-LOTIS has the ideal sensitivity and field-of-view to conduct novae and supernovae searches in nearby galaxies. The novae data will provide information about differences in stellar populations within galaxies while the rate provides information about the star formation history. Like GRBs, supernovae observations at early-times are very important to establish the rise characteristics. The nearby supernovae discovered by Super-LOTIS will be used as templates for the high-z events. 


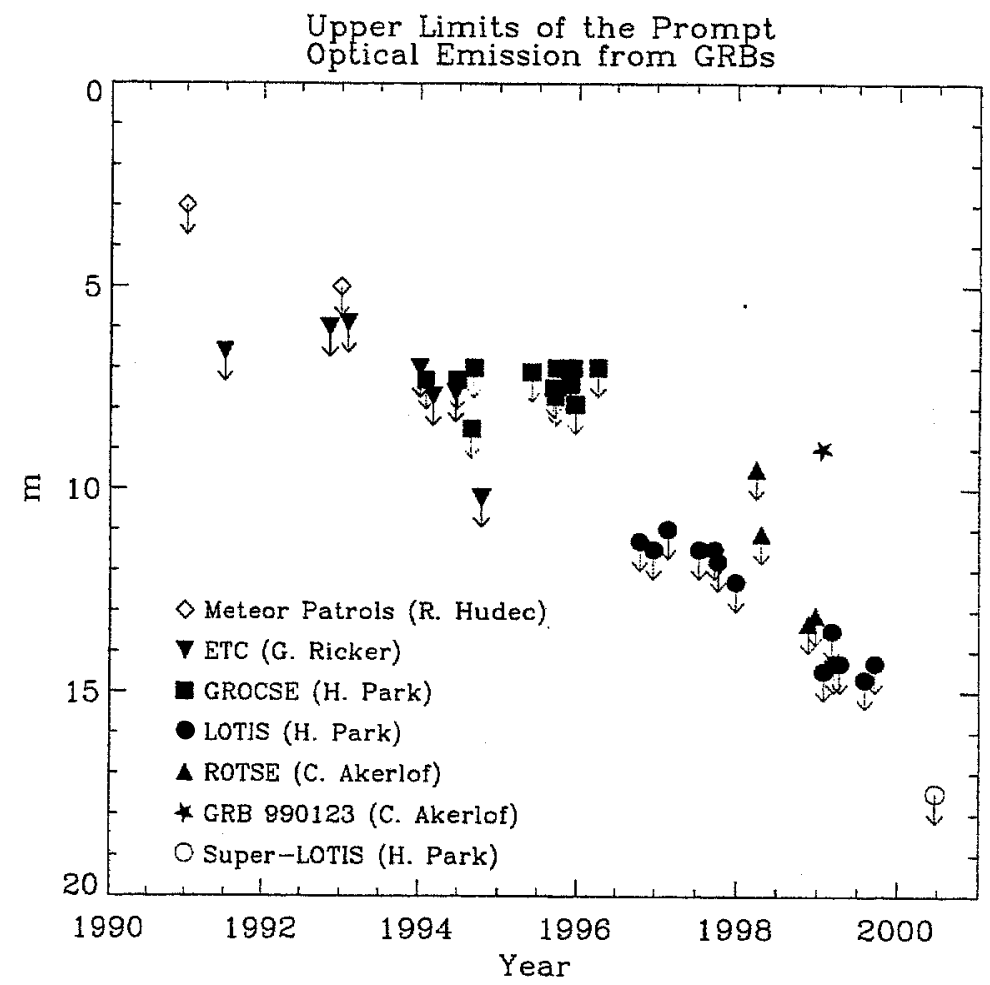

Figure 6.1 Upper limits of the prompt optical emission from GRBs. Data presented here and from published results. 


\section{REFERENCES}

Akerlof, C., Balsano, R., Barthelemy, S., Bloch, J., Butterworth, P., Casperson, D., Cline, T., Fletcher, S., Frontera, F., Gisler, G., Heise, J., Hills, J., Kehoe, R., Lee, B., Marshall, S., McKay, T., Miller, R., Piro, L., Priedhorsky, W., Szymanski, J., \& Wren, J. 1999, Nature, 398, 400

Akerlof, C., Balsano, R., Barthelmy, S., Bloch, J., Butterworth, P., Casperson, D., Cline, T., Fletcher, S., Frontera, F., Gisler, G., Heise, J., Hills, J., Hurley, K., Kehoe, R., Lee, B., Marshall, S., McKay, T., Pawl, A., Piro, L., Szymanski, J., \& Wren, J. 2000, ApJ, 532, L25

Amati, L., Cinti, M. N., Feroci, M., Costa, E., Frontera, F., dal Fiume, D., Collina, P., Nicastro, L., Orlandini, M., Palazzi, E., Rapisarda, M., \& Zavattini, G. 1997, Proc. SPIE, 3114, 176

Atteia, J. ., Barat, C., Hurley, K., Niel, M., Vedrenne, G., Wenzel, W., Fenimore, E. E., Klebesadel, R., Cline, T., \& Desai, U. 1985, A\&A, 152, 174

Attina', P., Alippi, E., Casoli, P., Lenti, A., Monzani, F., \& Poulsen, J. M. 1995, Proc. SPIE, 2517, 182

Baan, W. A. 1982, ApJ, 261, L71

Band, D., Matteson, J., Ford, L., Schaefer, B., Palmer, D., Teegarden, B., Cline, T., Briggs, M., Paciesas, W., Pendleton, G., Fishman, G., Kouveliotou, C., Meegan, C., Wilson, R., \& Lestrade, P. 1993, ApJ, 413, 281

Baring, M. G. \& Harding, A. K. 1997, ApJ, 491, 663+

Barthelmy, S. D., Cline, T. L., Butterworth, P. S., Kippen, R. M., Briggs, M., Connaughton, V., \& Pendleton, G. 2000, in Gamma-Ray Bursts, pp 99+

Barthelmy, S. D., Cline, T. L., Gehrels, N., Bialas, T. G., Robbins, M. A., Kuyper, J. R., Fishman, G. J., Kouveliotou, C., \& Meegan, C. A. 1994, in G. J. Fishman, J. J. Brainerd, and K. Hurley (eds.), Gamma-Ray Bursts, No. 307 in AIP Conference Proceedings, pp 643+

Barthelmy, S. D., Cline, T. L., Teegarden, B. J., \& von Rosenvinge, T. T. 1992, in ASP Conf. Ser. 34: Robotic Telescopes in the 1990s, pp 137+

Blaes, O. M. 1994, ApJS, 92, 643

Blandford, R. D. \& Znajek, R. L. 1977, MNRAS, 179, 433 
Bloom, J. S., Kulkarni, S. R., Djorgovski, S. G., Eichelberger, A. C., Cote, P., Blakeslee, J. P., Odewahn, S. C., Harrison, F. A., Frail, D. A., Filippenko, A. V., Leonard, D. C., Riess, A. G., Spinrad, H., Stern, D., Bunker, A., Dey, A., Grossan, B., Perlmutter, S., Knop, R. A., Hook, I. M., \& Feroci, M. 1999, Nature, 401, 453

Boella, G., Butler, R. C., Perola, G. C., Piro, L., Scarsi, L., \& Bleeker, J. A. M. 1997, A\&AS, 122, 299

Briggs, M. S., Band, D. L., Kippen, R. M., Preece, R. D., Kouveliotou, C., van Paradijs, J., Share, G. H., Murphy, R. J., Matz, S. M., Connors, A., Winkler, C., McConnell, M. L., Ryan, J. M., Williams, O. R., Young, C. A., Dingus, B., Catelli, J. R., \& Wijers, R. A. M. J. 1999a, ApJ, 524, 82

Briggs, M. S., Pendleton, G. N., Kippen, R. M., Brainerd, J. J., Hurley, K., Connaughton, V., \& Meegan, C. A. 1999b, ApJS, 122, 503

Butler, R. C., Piro, L., Costa, E., Feroci, M., Frontera, F., dal Fiume, D., Heise, J., Jager, R., Sacco, B., Chiappetti, L., Parmar, A., Antonelli, L. A., Ricci, D., Muller, J. M., Coletta, A., de Libero, C., Salotti, L., Voges, W., Boller, T., \& Greiner, J. 1997, IAU Circ., 6539, 1+

Castro-Tirado, A. 1998, in Ultraviolet Astrophysics Beyond the IUE Final Archive, pp 659+

Castro-Tirado, A. J., Brandt, S., Lund, N., \& Guziy, S. S. 1994, in G. J. Fishman, J. J. Brainerd, and K. Hurley (eds.), Gamma-Ray Bursts, No. 307 in AIP Conference Proceedings, pp 404+

Castro-Tirado, A. J., Gorosabel, J., Heidt, J., Seitz, T., Thommes, E., Wolf, C., Lund, N., Pedersen, H., Costa, E., Frontera, F., Heise, J., In't Zand, J., Bartolini, C., Guarnieri, A., Masetti, A., Piccioni, A., \& Palazzi, E. 1997, IAU Circ., $6598,2+$

Cavallo, G. \& Rees, M. J. 1978, MNRAS, 183, 359

Chevalier, C., Ilovaisky, S. A., Motch, C., Barat, C., Hurley, K., Niel, M., Vedrenne, G., Laros, J. G., Evans, W. D., \& Fenimore, E. E. 1981, A\&A, 100, L1

Cline, D. B. \& Hong, W. 1992, ApJ, 401, L57

Cline, T. L., Desai, U. D., Klebesadel, R. W., \& Strong, I. B. 1973, ApJ, 185, L1

Cline, T. L., Desai, U. D., Trainor, J. H., Pizzichini, G., Spizzichino, A., Klebesadel, R. W., \& Helmken, H. 1979, ApJ, 232, L1

Colgate, S. A. 1968, "Canadian J. Phys", 46, S476

Colgate, S. A. 1974, ApJ, 187, 333

Cortiglioni, S., Mandolesi, N., Morigi, G., Ciapi, A., Inzani, P., \& Sironi, G. 1981, Ap\&SS, 75, 153 
Costa, E., Feroci, M., Frontera, F., Zavattini, G., Nicastro, L., Palazzi, E., Spoliti, G., di Ciolo, L., Coletta, A., D'Andreta, G., Muller, J. M., Jager, R., Heise, J., \& In't Zand, J. 1997a, IAU Circ., 6572, 1+

Costa, E., Feroci, M., Piro, L., Cinti, M. N., Frontera, F., Zavattini, G., Nicastro, L., Palazzi, E., dal Fiume, D., Orlandini, M., In't Zand, J., Heise, J., Jager, R., Parmar, A., Owens, A., Molendi, S., Cusumano, G., Maccarone, M. C., Giarrusso, S., Antonelli, L. A., Fiore, F., Giommi, P., Muller, J. M., Salotti, L., Gennaro, G., Stornelli, M., Crisigiovanni, G., Ricci, R., Coletta, A., Butler, R. C., Frail, D. A., \& Kulkarni, S. R. 1997b, IAU Circ., 6576, 1+

Costa, E., Feroci, M., Piro, L., Frontera, F., Zavattini, G., Nicastro, L., Palazzi, E., Gandolfi, G., Smith, M., Coletta, A., D'Andreta, G., Spoliti, G., Jager, R., \& Heise, J. 1997c, IAU Circ., 6533, 1+

Crider, A., Liang, E. P., Smith, I. A., Preece, R. D., Briggs, M. S., Pendleton, G. N., Paciesas, W. S., Band, D. L., \& Matteson, J. L. 1997, ApJ, 479, L39

Dar, A. 1998, ApJ, 500, L93

Eichler, D., Livio, M., Piran, T., \& Schramm, D. N. 1989, Nature, 340, 126

Fenimore, E. E., Madras, C. D., \& Nayakshin, S. 1996, ApJ, 473, $998+$

Fenimore, E. E. \& Ramirez-Ruiz, E. 2000, ApJ, 000, 0

Feroci, M., Frontera, F., Costa, E., dal Fiume, D., Amati, L., Bruca, L., Cinti, M. N., Coletta, A., Collina, P., Guidorzi, C., Nicastro, L., Orlandini, M., Palazzi, E., Rapisarda, M., Zavattini, G., \& Butler, R. C. 1997, Proc. SPIE, 3114, 186

Fisher, N. I., Lewis, T., \& Embleton, B. J. J. 1987, "Statistical analysis of spherical data", Cambridge: University Press, 1987

Fishman, G. J. 1995, PASP, 107, 1145+

Fishman, G. J., Duthie, J. G., \& Dufour, R. J. 1981, Ap\&SS, 75, 135

Fishman, G. J. \& Hartmann, D. H. 1997, Scientific American, 277, 34

Fishman, G. J. \& Meegan, C. A. 1995, ARA\&A, 33, 415

Fishman, G. J., Meegan, C. A., Wilson, R. B., Brock, M. N., Horack, J. M., Kouveliotou, C., Howard, S., Paciesas, W. S., Briggs, M. S., Pendleton, G. N., Koshut, T. M., Mallozzi, R. S., Stollberg, M., \& Lestrade, J. P. 1994, ApJS, 92,229

Fishman, G. J., Meegan, C. A., Wilson, R. B., Paciesas, W. S., \& Pendleton, G. N. 1992, in The Compton Observatory Science Workshop, pp 26-34

Ford, L. A. \& Band, D. L. 1996, ApJ, 473, 1013+ 
Ford, L. A., Band, D. L., Matteson, J. L., Briggs, M. S., Pendleton, G. N., Preece, R. D., Paciesas, W. S., Teegarden, B. J., Palmer, D. M., Schaefer, B. E., Cline, T. L., Fishman, G. J., Kouveliotou, C., Meegan, C. A., Wilson, R. B., \& Lestrade, J. P. 1995, ApJ, 439, 307

Frail, D. A., Halpern, J. P., Bloom, J. S., Kulkarni, S. R., \& G., D. S. 1998, GCN Circ. 128, pp 1+

Frail, D. A., Kulkarni, S. R., Nicastro, L., dal Fiume, D., Orlandini, M., Palazzi, E., Pizzichini, G., Frontera, F., Zavattini, G., Amati, L., Cinti, M. N., Costa, E., Feroci, M., Piro, L., Jager, R., Heise, J., Hurley, K., Kouveliotou, C., Fishman, G., \& Meegan, C. 1997, IAU Circ., 6545, 1+

Frail, D. A., Kulkarni, S. R., Piro, L., Costa, E., Feroci, M., Cinti, M., Frontera, F., dal Fiume, D., Nicastro, L., Orlandini, M., Pizzichini, G., Heise, J., Jager, R., \& Muller, H. 1996, IAU Circ., 6472, 2+

Frontera, F., Costa, E., dal Fiume, D., Feroci, M., Nicastro, L., Orlandini, M., Palazzi, E., \& Zavattini, G. 1997, A\&AS, 122, 357

Fryer, C., Burrows, A., \& Benz, W. 1998, ApJ, 496, 333+

Fryer, C. L., Woosley, S. E., \& Hartmann, D. H. 1999, ApJ, 526, 152

Galama, T., Groot, P. J., \& van Paradijs, J. 1997, IAU Circ., 6574, 1+

Galama, T. J., van Paradijs, J., Antonelli, L. A., Vreeswijk, P. M., Kouveliotou, C., Torroni, V., \& Pastor, C. 1998a, GCN Circ. 127, pp 1+

Galama, T. J., Vreeswijk, P. M., Pian, E., Frontera, F., Doublier, V., Gonzalez, J. ., Lidman, C., Augusteijn, T., Hainaut, O. R., Boehnhardt, H., Patat, F., \& Leibundgut, B. 1998b, IAU Circ., 6895, 1+

Gilman, D., Metzger, A. E., Parker, R. H., Evans, L. G., \& Trombka, J. I. 1980, ApJ, 236,951

Golenetskii, S. V., Mazets, E. P., Aptekar, R. L., \& Ilinskii, V. N. 1983, Nature, 306, 451

Goodman, J. 1986, ApJ, 308, L47

Greiner, J. 1992, A\&A, 264, 121

Greiner, J. 1995, Ap\&SS, 231, 263

Greiner, J., Flohrer, J., Wenzel, W., \& Lehmann, T. 1987, Ap\&SS, 138, 155

Greiner, J., Hagen, H. ., \& Heines, A. 1996a, IAU Circ., 6487, $1+$

Greiner, J. \& Motch, C. 1995, A\&A, 294, 177

Greiner, J., Wenzel, W., \& Degel, W. 1990, A\&A, 234, 251 
Greiner, J., Wenzel, W., Hudec, R., Spurny, P., Florian, J., Ziener, R., Moskalenko, E. I., Barabanov, A. V., Chernych, N. S., Birkle, K., Bade, N., Tritton, S. B., Ichikawa, T., Kouveliotou, C., Fishman, G. J., Meegan, C. A., Paciesas, W. S., \& Wilson, R. B. 1996b, in C. Kouveliotou, M. F. Briggs, and G. J. Fishman (eds.), Gamma-Ray Bursts, No. 384 in AIP Conference Proceedings, pp 565+

Grindlay, J. E. \& Fazio, G. G. 1974, ApJ, 187, L93

Grindlay, J. E., Wright, E. L., \& McCrosky, R. E. 1974, ApJ, 192, L113

Groot, P. J., Galama, T. J., van Paradijs, J., Kouveliotou, C., Wijers, R. A. M. J., Bloom, J., Tanvir, N., Vanderspek, R., Greiner, J., Castro-Tirado, A. J., Gorosabel, J., von Hippel, T., Lehnert, M., Kuijken, K., Hoekstra, H., Metcalfe, N., Howk, C., Conselice, C., Telting, J., Rutten, R. G. M., Rhoads, J., Cole, A., Pisano, D. J., Naber, R., \& Schwarz, R. 1998, ApJ, 493, L27

Groot, P. J., Galama, T. J., van Paradijs, J., Strom, R., Telting, J., Rutten, R. G. M., Pettini, M., Tanvir, N., Naber, R., Kouveliotou, C., In't Zand, J., Heise, J., Costa, E., Feroci, M., Piro, L., Frontera, F., Zavattini, G., Nicastro, L., \& Palazzi, E. 1997, IAU Circ., 6584, 1+

Guarnieri, A., Bartolini, C., Piccioni, A., Masetti, N., Teodorani, M., Costa, E., dal Fiume, D., \& Frontera, F. 1997, IAU Circ., 6544, 1+

Harrison, F. A., Bloom, J. S., Frail, D. A., Sari, R., Kulkarni, S. R., Djorgovski, S. G., Axelrod, T., Mould, J., Schmidt, B. P., Wieringa, M. H., Wark, R. M., Subrahmanyan, R., McConnell, D., McCarthy, P. J., Schaefer, B. E., McMahon, R. G., Markze, R. O., Firth, E., Soffitta, P., \& Amati, L. 1999, ApJ, 523, L121

Harrison, T. E., McNamara, B. J., \& Klemola, A. 1994a, AJ, 107, 254

Harrison, T. E., McNamara, B. J., \& Klemola, A. R. 1994b, AJ, 108, 600

Hartmann, D. \& Epstein, R. I. 1989, ApJ, 346, 960

Hartmann, D. H. 1995, in Flares and Flashes; Proceedings of the IAU Colloquium No. 151; Held in Sonneberg; Germany; 5 - 9 December 1994; XXII; 477 pp.. Springer-Verlag Berlin Heidelberg New York. Also Lecture Notes in Physics; volume 454 Edited by Jochen Greiner, Hilmar W. Duerbeck, and Roald E. Gershberg, p.367, pp 367+

Hartmann, D. H., Williams, G. G., Band, D., Hurley, K., \& Park, H. . 1998, in Abstracts of the 19th Texas Symposium on Relativistic Astrophysics and Cosmology, held in Paris, France, Dec. 14-18, 1998. Eds.: J. Paul, T. Montmerle, and E. Aubourg (CEA Saclay)., pp E24-+

Herzo, D., Zych, A. D., White, R. S., \& Dayton, B. 1976, ApJ, 203, L115

Hjellming, R. M. \& Ewald, S. P. 1981, ApJ, 246, L137 
Holcomb, K. A. \& Tajima, T. 1991, ApJ, 378, 682

Hoyle, F. \& Clayton, D. D. 1974, ApJ, 191, 705

Hudec, R. 1993a, A\&AS, 97, 49

Hudec, R. 1993b, Astrophys. Lett., 28, 359

Hudec, R. 1995, Ap\&SS, 231, 239

Hudec, R., Borovicka, J., Wenzel, W., Atteia, J. ., Barat, C., Hurley, K., Niel, M., Cline, T., Desai, U., \& Teegarden, B. 1987, A\&A, 175, 71

Hudec, R., Dedoch, A., Pravec, P., \& Borovicka, J. 1994, A\&A, 284, 839

Hurley, K. C. 1998, in C. A. Meegan, R. D. Preece, and T. M. Koshut (eds.), Gamma-Ray Bursts, No. 428 in AIP Conference Proceedings, pp 87+

In't Zand, J., Heise, J., Hoyng, P., Jager, R., Piro, L., Costa, E., Feroci, M., Frontera, F., dal Fiume, D., Orlandini, M., Pizzichini, G., Nicastro, L., \& Muller, J. 1997, IAU Circ., 6569, 1+

Jenkner, H., Lasker, B. M., Sturch, C. R., McLean, B. J., Shara, M. M., \& Russel, J. L. 1990, AJ, 99, 2082

Klebesadel, R. W., Strong, I. B., \& Olson, R. A. 1973, ApJ, 182, L85

Kouveliotou, C., Meegan, C. A., Fishman, G. J., Bhat, N. P., Briggs, M. S., Koshut, T. M., Paciesas, W. S., \& Pendleton, G. N. 1993, ApJ, 413, L101

Krimm, H. A., Vanderspek, R. K., \& Ricker, G. R. 1996, A\&AS, 120, C251

Krolik, J. H. \& Pier, E. A. 1991, ApJ, 373, 277

Lamb, D. Q. 1973, Nature, 246, PS52

Lamb, D. Q. \& Reichart, D. E. 2000, ApJ, 536, 1

Lee, B., Akerlof, C., Band, D., Barthelmy, S., Butterworth, P., Cline, T., Ferguson, D., Gehrels, N., \& Hurley, K. 1997, ApJ, 482, L125

Lee, H. K., Wijers, R. A. M. J., \& Brown, G. E. 1999, in ASP Conf. Ser. 190: Gamma-Ray Bursts: The First Three Minutes, pp 173+

Levine, A., Morgan, E., \& Muno, M. 1998, IAU Circ., 6966, 1+

Liang, E. P. \& Antiochos, S. K. 1984, Nature, 310, 121+

Liang, E. P., Crider, A., Böttcher, M., \& Smith, I. A. 1999, ApJ, 519, L21

Luginbuhl, C., Vrba, F., Hudec, R., Pravec, P., Sarounova, L., Greiner, J., \& Tovmassian, G. 1996, IAU Circ., 6526, 1+

MacFadyen, A. I. \& Woosley, S. E. 1999, ApJ, 524, 262 
Masetti, N., Palazzi, E., Pian, E., Hunt, L. K., Méndez, M., Frontera, F., Amati, L., Vreeswijk, P. M., Rol, E., Galama, T. J., van Paradijs, J., Antonelli, L. A., Nicastro, L., Feroci, M., Marconi, G., Piro, L., Costa, E., Kouveliotou, C., Castro-Tirado, A. J., Falomo, R., Augusteijn, T., Böhnhardt, H., Lidman, C., Vanzi, L., Merrill, K. M., Kaminsky, C. D., van der Klis, M., Heemskerk, M. H. M., van der Hooft, F., Kuulkers, E., Pedersen, H., \& Benetti, S. 2000, A\&A, 354, 473

Mazets, E. P., Golenetskii, S. V., Aptekar, R. L., Gurian, I. A., \& Ilinskii, V. N. 1981, Nature, 290,378

McNamara, B. \& Harrison, T. E. 1994, AJ, 107, 1825

Meegan, C. A., Pendleton, G. N., Briggs, M. S., Kouveliotou, C., Koshut, T. M., Lestrade, J. P., Paciesas, W. S., McCollough, M. L., Brainerd, J. J., Horack, J. M., Hakkila, J., Henze, W., Preece, R. D., Mallozzi, R. S., \& Fishman, G. J. 1996, ApJS, 106, 65+

Meszaros, P. \& Rees, M. J. 1993, ApJ, 405, 278

Mészáros, P. \& Rees, M. J. 1999, MNRAS, 306, L39

Monet, D. 1996, "USNO-A1.0 Catalog (Washington DC: USNO)"

Moskalenko, E. I., Popravko, G. V., Kramer, E. N., Shestaka, I. S., Karnashov, A. N., Nazarenko, V. V., Skoblikova, L. J., Lemeshchenko, V. F., Nazarenko, S. V., \& Gorbanev, J. M. 1989, A\&A, 223, 141

Murakami, T., Fujii, M., Hayashida, K., Itoh, M., \& Nishimura, J. 1988, Nature, 335, $234+$

Murakami, T., Shibata, R., Inoue, H., Nagase, F., Yoshida, A., Kawai, N., Otani, C., Tokanai, F., \& Heise, J. 1996, IAU Circ., 6481, 1+

Muslimov, A. G. \& Tsygan, A. I. 1986, Ap\&SS, 120, 27

Nemiroff, R. J. 1994, "Comments on Astrophysics", 17, 189

Newman, M. J. \& Cox, A. N. 1980, ApJ, 242, 319

Norris, J. P., Marani, G. F., \& Bonnell, J. T. 2000, ApJ, 534, 248

Norris, J. P., Share, G. H., Messina, D. C., Dennis, B. R., Desai, U. D., Cline, T. L., Matz, S. M., \& Chupp, E. L. 1986, ApJ, 301, 213

Pacini, F. 1974, Nature, 251, 399

Paczynski, B. 1998, in Gamma-Ray Bursts, pp 783+

Park, H., Ables, E., Barthelmy, S. D., Bionta, R. M., Ott, L. L., Parker, E. L., \& Williams, G. G. 1998a, Proc. SPIE, 3355, 658 
Park, H. S., Ables, E., Band, D. L., Barthelmy, S. D., Bionta, R. M., Butterworth, P. S., Cline, T. L., Ferguson, D. H., Fishman, G. J., Gehrels, N., Hurley, K., Kouveliotou, C., Lee, B. C., Meegan, C. A., Ott, L. L., \& Parker, E. L. 1997a, ApJ, 490, 99+

Park, H. S., Bionta, R., Ables, E., Ott, L., Parker, E., Williams, G. G., Hartmannn, D. H., Barthelmy, S. D., Gehrels, N., Butterworth, P. S., Cline, T. L., Kouveliotou, C., Fishman, G. J., Meegan, C. A., Band, D. L., Hurley, K., \& Ferguson, D. H. 1997b, GCN Circ. 019, pp 1+

Park, H. S., Ott, L., Porrata, R. A., Williams, G. G., \& Hartmann, D. H. 1999a, GCN Circ. 277, pp 1+

Park, H. S., Porrata, R. A., \& Williams, G. G. 1998b, GCN Circ. 135, pp 1+

Park, H. S., Porrata, R. A., Williams, G. G., Ables, E., Band, D. L., Barthelmy, S. D., Bionta, R. M., Cline, T. L., Fishman, G. J., Gehrels, N., Hartmann, D., Hurley, K., Kouveliotou, C., \& Meegan, C. A. 1999b, A\&AS, 138, 577

Park, H. S., Williams, G. G., Ables, E., Band, D. L., Barthelmy, S. D., Bionta, R., Butterworth, P. S., Cline, T. L., Ferguson, D. H., Fishman, G. J., Gehrels, N., Hartmann, D., Hurley, K., Kouveliotou, C., Meegan, C. A., Ott, L., Parker, E., \& Wurtz, R. 1997c, ApJ, 490, L21

Pedersen, H., Motch, C., Tarenghi, M., Danziger, J., Pizzichini, G., \& Lewin, W. H. G. 1983, ApJ, 270, L43

Pedersen, H., Tarenghi, M., Grosbol, P., Danziger, J., Pizzichini, G., Lewin, W. H. G., \& Liller, M. 1982, IAU Circ., 3711, 1+

Piran, T. 1999a, in ASP Conf. Ser. 190: Gamma-Ray Bursts: The First Three Minutes, pp 3+

Piran, T. 1999b, Phys. Rep., 314, 575+

Piro, L., Costa, E., Feroci, M., Cinti, M., Frontera, F., dal Fiume, D., Nicastro, L., Orlandini, M., Pizzichini, G., Heise, J., Jager, R., Fiore, F., Giommi, P., Matteuzzi, A., Muller, H., Parmar, A., Sacco, B., Molendi, S., \& Butler, R. C. 1996a, IAU Circ., 6480, $1+$

Piro, L., Costa, E., Feroci, M., Cinti, M., Frontera, F., dal Fiume, D., Orlandini, M., Pizzichini, G., Heise, J., Jager, R., \& Muller, H. 1996b, IAU Circ., 6467, 1+

Piro, L., Scarsi, L., \& Butler, R. C. 1995, Proc. SPIE, 2517, 169

Portegies Zwart, S. F. \& Spreeuw, H. N. 1996, A\&A, 312, 670

Ramaty, R., Bonazzola, S., Cline, T. L., Kazanas, D., Meszaros, P., \& Lingenfelter, R. E. 1980, Nature, 287, 122

Rees, M. J. \& Meszaros, P. 1992, MNRAS, 258, 41P 
Rees, M. J. \& Meszaros, P. 1994, ApJ, 430, L93

Reichart, D. E. 1998, ApJ, 495, L99

Reichart, D. E. 1999, ApJ, 521, L111

Ricker, G. R., Mock, P. C., Ajhar, E. A., \& Vanderspek, R. K. 1989, ApJ, 338, 983

Ruderman, M. 1975, New York Academy Sciences Annals, 262, 164

Salmonson, J. D. 2000, ApJ, 000, 0

Sari, R. \& Piran, T. 1997, ApJ, 485, 270+

Sari, R. \& Piran, T. 1999a, ApJ, 517, L109

Sari, R. \& Piran, T. 1999b, ApJ, 520, 641

Sari, R., Piran, T., \& Narayan, R. 1998, ApJ, 497, L17

Schaefer, B. E. 1981, Nature, 294, 722

Schaefer, B. E., Barthelmy, S. D., Palmer, D. M., Cline, T. L., Hurley, K. C., Sommer, M., Boer, M., Niel, M., Fishman, G. J., Kouveliotou, C., Meegan, C. A., Coe, M. J., Elmegreen, B. G., McNamara, B. J., Harrison, T., Owens, A., Sollee, N., Stull, J., Palermiti, M., Schwartz, R., Brooks, J., Vanderspek, R., Ricker, G. R., Krimm, H. A., Wenzel, W., Greiner, J., Hudec, R., Novak, M., Borovicka, J., Predota, M., Bailyn, C. D., Hanlon, L. O., Bennett, K., Spoelstra, T. A. T., Smette, A., Pedersen, H., Florentin, R., Pollas, C., Steinle, H., Schramm, K. ., Iyengar, K. V. K., \& Prabhu, T. P. 1994a, ApJ, 422, L71

Schaefer, B. E., Bradt, H. V., Barat, C., Hurley, K., Niel, M., Vedrenne, G., Cline, T. L., Desai, U., Teegarden, B. J., Evans, W. D., Fenimore, E. E., Klebesadel, R. W., Laros, J. G., Estulin, I. V., \& Kuznetsov, A. V. 1984, ApJ, 286, L1

Schaefer, B. E., Cline, T. L., Hurley, K. C., \& Laros, J. G. 1997, ApJ, 489, 693+

Schaefer, B. E. \& Ricker, G. R. 1983, Nature, 302, 43

Schaefer, B. E., Seitzer, P., \& Bradt, H. 1983, ApJ, 270, L49

Schaefer, B. E., Snyder, J. A., Hernandez, J., Roscherr, B., Deng, M., Ellman, N., Bailyn, C., Rengstorf, A., Smith, D., Levine, A., Barthelmy, S., Butterworth, P., Hurley, K., Cline, T., Meegan, C., Kouveliotou, C., Kippen, R. M., Park, H. ., Williams, G. G., Porrata, R., Bionta, R., Hartmann, D., Band, D., Frail, D., Kulkarni, S., Bloom, J., Djorgovski, S., Sadava, D., Chaffee, F., Harris, F., Abad, C., Adams, B., Andrews, P., Baltay, C., Bongiovanni, A., Briceno, C., Bruzual, G., Coppi, P., della Prugna, F., Dubuc, A., Emmet, W., Ferrin, I., Fuenmayor, F., Gebhard, M., Herrera, D., Honeycutt, K., Magris, G., Mateu, J., Muffson, S., Musser, J., Naranjo, O., Oemler, A., Pacheco, R., Paredes, G., Rengel, M., Romero, L., Rosenzweig, P., Sabbey, C., Sánchez, G., Sánchez, G., Schenner, H., Shin, J., Sinnott, J., Sofia, S., Stock, J., Suarez, J., Telléria, D., Vicente, B., Vieira, K., \& Vivas, K. 1999, ApJ, 524, L103 
Schaefer, B. E., Teegarden, B. J., Fantasia, S. F., Palmer, D., Cline, T. L., Matteson, J. L., Band, D. L., Ford, L. A., Fishman, G. J., Meegan, C. A., Wilson, R. B., Paciesas, W. S., Pendleton, G. N., Briggs, M. S., \& Lestrade, J. P. 1994b, ApJS, 92, 285

Scholz, M. 1984, Informational Bulletin on Variable Stars, 2615, $1+$

Schwartz, R. A., Ling, J. C., Mahoney, W. A., \& Jacobson, A. S. 1987, ApJ, 317, 846

Shemi, A. \& Piran, T. 1990, ApJ, 365, L55

Smith, D. A., Levine, A. M., \& Muno, M. 1998, GCN Circ. 126, pp $1+$

Smith, D. A., Levine, A. M., \& Remillard, R. 1999, GCN Circ. 275, pp $1+$

Sokolov, V. V., Kurt, V. G., Kopylov, A. I., Zharikov, S. V., \& Berezin, A. V. 1996 , Astronomy Letters, 22, 502

Taylor, J. H., Fowler, L. A., \& McCulloch, P. M. 1979, Nature, 277, 437

Taylor, J. H. \& Weisberg, J. M. 1982, ApJ, 253, 908

Totani, T. 1999, ApJ, 511, 41

Vanderspek, R. K., Ricker, G. C., \& Doty, J. P. 1992, in ASP Conf. Ser. 34: Robotic Telescopes in the 1990s, pp 123+

Vrba, F. J. 1996, in C. Kouveliotou, M. F. Briggs, and G. J. Fishman (eds.), GammaRay Bursts, No. 384 in AIP Conference Proceedings, pp 565+

Vrba, F. J., Hartmann, D. H., \& Jennings, M. C. 1995, ApJ, 446, 115+

Vreeswijk, P. M., Galama, T. J., Lipkin, Y., Leibowitz, E., Lidman, C., Doublier, V., van Paradijs, J., Kouveliotou, C., \& Robinson, C. 1998, GCN Circ. 132, pp $1+$

Vreeswijk, P. M., Galama, T. J., Owens, A., Oosterbroek, T., Geballe, T. R., van Paradijs, J., Groot, P. J., Kouveliotou, C., Koshut, T., Tanvir, N., Wijers, R. A. M. J., Pian, E., Palazzi, E., Frontera, F., Masetti, N., Robinson, C., Briggs, M., In't Zand, J. J. M., Heise, J., Piro, L., Costa, E., Feroci, M., Antonelli, L. A., Hurley, K., Greiner, J., Smith, D. A., Levine, A. M., Lipkin, Y., Leibowitz, E., Lidman, C., Pizzella, A., Böhnhardt, H., Doublier, V., Chaty, S., Smail, I., Blain, A., Hough, J. H., Young, S., \& Suntzeff, N. 1999, ApJ, 523, 171

Walker, K. C., Schaefer, B. E., \& Fenimore, E. E. 2000, ApJ, 537, 264

Wang, J. C. L., Lamb, D. Q., Loredo, T. J., Wasserman, I. M., \& Salpeter, E. E. 1989, Physical Review Letters, 63, 1550 
Wijers, R. A. M. J., Bloom, J. S., Bagla, J. S., \& Natarajan, P. 1998, MNRAS, 294, L13

Williams, G. G., Park, H. S., Ables, E., Band, D. L., Barthelmy, S. D., Bionta, R., Butterworth, P. S., Cline, T. L., Ferguson, D. H., Fishman, G. J., Gehrels, N., Hartmann, D. H., Hurley, K., Kouveliotou, C., Meegan, C. A., Ott, L., Parker, E., \& Porrata, R. 1999a, ApJ, 519, L25

Williams, G. G., Park, H. S., Ables, E., Band, D. L., Barthelmy, S. D., Bionta, R., Butterworth, P. S., Cline, T. L., Ferguson, D. H., Fishman, G. J., Gehrels, N., Hartmann, D. H., Hurley, K., Kouveliotou, C., Meegan, C. A., Ott, L., Parker, E., \& Wurtz, R. 1998, in C. A. Meegan, R. D. Preece, and T. M. Koshut (eds.), Gamma-Ray Bursts, No. 428 in AIP Conference Proceedings, pp $837+$

Williams, G. G., Park, H. S., \& Porrata, R. A. 1999b, GCN Circ. 272, pp $1+$

Woosley, S. E. 1993, ApJ, 405, 273

Woosley, S. E. \& Wallace, R. K. 1982, ApJ, 258, 716

Zapatero Osorio, M. R., Castro-Tirado, A., Gorosabel, J., Oscoz, A., Kemp, S., Frontera, F., \& Nicastro, L. 1998, IAU Circ., 6967, 1+

Zytkow, A. N. 1990, ApJ, 359, 138 